GSA Data Repository 2017055

\title{
Quantifying the rise of the Himalaya orogen and implications for the South Asian monsoon
}

Lin Ding, R. A. Spicer, Jian Yang, Qiang Xu, Fulong Cai, Shun Li, Qingzhou Lai, Houqi Wang, T. E.V. Spicer, Yahui Yue, A. Shukla, G. Srivastava, M. Ali Khan, S. Bera, and R. Mehrotra

\section{S1 Geological and sedimentary settings}

\section{S1.1 Liuqu Formation}

The Liuqu Formation outcrops are entirely restricted to an elongate zone along the south of Yarlung-Zangpo suture (YZS). The conglomerate extends from north of the town of Lhaze to south of Xigatze along a distance of $150 \mathrm{~km}$, varies in width between hundreds of meters to kilometers and occupies a total area of about $30 \mathrm{~km}^{2}$

(Fig. 2) (Davis et al., 2002; Fang et al., 2006; Aitchison et al., 2009, 2011; Wang et al., 2010).

Our investigated sections extend south-north across the conglomerate at Gongjiongla village, east of Lhaze town (Fig. 2A) and Za village (Fig. 2B) near Liuqu village and near Xialu village nearby Xigatze (Fig. 2C). The conglomerates are in fault contact with Yarlung-Zangbo ophiolites to the north and the Triassic-Cretaceous low-grade metasedimentary rocks of the passive continental margin sedimentary rocks 
of the Tethyan succession to the south (Fig. 2F). The Liuqu Formation was deposited in a series of narrow elongate basins between the two faults. With a total thickness over $3500 \mathrm{~m}$ the strata generally consist of mottled massive conglomerates with a small portion of purple red sandstones intercalated with mudstones (Fig. 2E; Davis et al., 2002; Wang et al., 2010).

The lower part of the conglomerate measured section (Fig. 2E) is about 100-m-thick and represents an upward fining succession. It is composed of well-rounded clast-supported pebble to cobble conglomerates and thick-bedded trough cross-bedded sandy conglomerates in the lower part to homogeneous silt and mudstones in the upper part. The clasts are mainly composed of diorite in the base (Fig. DR4), and serpentinites, ultramafic rocks and gabbros in the overlying part. Sandstones form the transition to about $100 \mathrm{~m}$ of laminated mudstone beds.

A $30-\mathrm{cm}$ thick white tuffite layer has been found from the lower red part of the Liuqu Formation in the Xialu section (Fig. 2E). The tuffite samples were investigated by electron probe microanalyzer (EPMA). The backscatter scanning image clearly shows the sample mainly contains volcanic glass (Fig. DR3). Clay minerals such as illiite and chlorite are present around the glass shards. Quartz, dolomite and calcite are dominated in some thin beds. The major-element composition of the glass shards varied with great ranges, with high $\mathrm{SiO}_{2}$ contain ranging from 63 to 93 wt.\% and simultaneous contains of variable $\mathrm{Al}_{2} \mathrm{O}_{3} 2.5-10$ wt.\%, $\mathrm{MgO}$ 0.5-4 wt.\%, FeO 1-5 wt.\%, $\mathrm{K}_{2} \mathrm{O}$ 0.5-1.7 wt.\%, $\mathrm{CaO}$ 0.2-5.5 wt.\% and $\mathrm{TiO}_{2}$ 0.01-0.8 wt.\% (Table DR2). 
The textures and compositions of the glass shards show that they have weathered to induce dealumination and to form clay minerals dominated by illiite and chlorite.

In the Liuqu Formation, only one fossil layer was found in the succession and forms a marker bed for correlation between different measured sections. A quantity of plant megafossils comprising 36 species (representative specimens are shown in Figure DR1 were found in the siltstone beds from three parts of the measured sections. These fining-upward successions are interpreted to record progressive decrease in flow strength during deposition. Repetition of these successions possibly reflects deposition in a braided river environment. The diorite clasts in the base of the Liuqu Formation suggest that these clasts were from Gangdese arc (Fig. DR4). Their $\mathrm{U}-\mathrm{Pb}$ zircon ages range between 96 and $210 \mathrm{Ma}$ are within the age range of the Gangdese magmatic zircons (Ji et al., 2009), but are significantly different from the Himalayan age patterns (Wang et al., 2010). This is further evidence for the base of Liuqu conglomerate being derived from the Gangdese arc.

The upper part of the Liuqu Formation contains abundant of metamorphic rocks, such as quartzite from the Tethyan Himalaya successions. We interpret that the wedge top of the Gangba-Dingri foreland basin ( $\sim 52-45 \mathrm{Ma})$ was eroded and supplied detritus for the Liuqu Formation (Najman, et al., 2010; DeCelles et al., 2014).

\section{S 1.2 Qiuwu Formation}

The Qiabulin section is just west of Xigatze (Figs. 2D and 2F). The measured section can be separated into two parts based on lithology and contacts (Fig. 2E). 
The lower unit, the Qiuwu Formation, is about $280 \mathrm{~m}$ thick and comprises $10 \mathrm{~m}$ of volcanic and granitic pebbly conglomerate beds at the base followed by grey interlayered sandstones, coarse sandstones and mudstones with locally thinly-bedded coals. Cross-bedding in the sandstone beds indicates southward flow and contains leaf fossils (Figs. 2E and DR5).

The upper unit is Dazuqu Formation, which disconformably overlies the Qiuwu Formation, which is about 1060 m thick composed of mottled conglomerates, grey-yellow coarse sandstones, medium sandstones, purple mudstones and shale (Fig. 2E). Clasts of volcanic rocks, granitic rocks, carbonates and silicates are imbricated and tabular cross-bedding in the sandstones indicates northward paleocurrents.

Conglomerates of granites and volcanic clasts are likely derived from the Gangdese arc. Chert and ophiolite clasts occur in the base of a contemporary succession in the Kailash area and may be derived from the Yarlung-Zangpo suture and Tethyan Himalaya.

\section{S2 Zircon U-Pb dating and depositional age constraints}

We sampled 3 sandstones from the Liuqu Formation at Za section in the Liuqu area, one tuffite sample and diorite clasts at the Xialu section (Fig. DR3), and 9 sandstone samples from Qiuwu and Dazhuqu Formations (Fig. 2E; Table DR1) in the Qiabulin area. These samples are used to constrain depositional timing. 
Using standard density and magnetic separation techniques, zircons were selected from sandstone samples. Zircons were embedded stochastically in epoxy, polished down to half thickness, and then used for microscopic studies and LA-ICPMS analysis. U-Pb geochronology of zircons was conducted by a laser-ablation inductively-coupled-plasma mass spectrometry (LA-ICP-MS) at the Key Laboratory of Continental Collision and Plateau Uplift, Institute of Tibetan Plateau Research, Chinese Academy of Sciences. The analyses involve ablation of zircon with an ATL $193 \mathrm{~nm}$ ArF excimer laser ablation system (UP193FX) with spot sizes of $35 \mu \mathrm{m}$. The ablated sample aerosols were transported to the plasma source of the Nu Plasma HR ICP-MS in Helium carrier gas. All measurements were made in static mode, using Faraday detectors with $3 \times 10^{11}$ ohm resistors for ${ }^{238} \mathrm{U},{ }^{232} \mathrm{Th}$, ${ }^{208} \mathrm{~Pb}-{ }^{206} \mathrm{~Pb}$, and discrete dynode ion counters for ${ }^{204} \mathrm{~Pb}$ and ${ }^{202} \mathrm{Hg}$. The errors in determining ${ }^{206} \mathrm{~Pb} /{ }^{238} \mathrm{U}$ and ${ }^{206} \mathrm{~Pb} /{ }^{204} \mathrm{~Pb}$ result in $\sim 1-2 \%$ (at $2 \sigma$ ) in the ${ }^{206} \mathrm{~Pb} /{ }^{238} \mathrm{U}$ ages. For every five sample analyses, Plesovice (337 $\pm 0.37 \mathrm{Ma})$ (Slama et al., 2008) natural zircons were analyzed as external standards for matrix-matched calibration of U-Pb dating, and a NIST SRM 612 reference glass was analyzed as external standard for calibration of trace-element contents.

The errors in measurement of ${ }^{206} \mathrm{~Pb} /{ }^{207} \mathrm{~Pb}$ and ${ }^{206} \mathrm{~Pb} /{ }^{204} \mathrm{~Pb}$ also result in $\sim 1-2 \%$ (at $2 \sigma$ level) uncertainty in age for grains that are $>1.0 \mathrm{Ga}$, but are substantially larger for younger grains due to low intensity of the ${ }^{207} \mathrm{~Pb}$ signal. Zircon ages $<1.0 \mathrm{Ga}$ are reported as ${ }^{206} \mathrm{~Pb} /{ }^{238} \mathrm{U}$ ages, whereas ages $>1.0 \mathrm{Ga}$ are reported as ${ }^{206} \mathrm{~Pb} /{ }^{207} \mathrm{~Pb}$ ages. Common- $\mathrm{Pb}$ corrections and $\mathrm{U}-\mathrm{Pb}$ ages were calculated using ComPbCorr\# 
3.17 (Anderson, 2002). Uncertainties are at the $1 \sigma$ level, and include only measurement errors. Analyses that are $>20 \%$ discordant (by comparison of ${ }^{206} \mathrm{~Pb} /{ }^{238} \mathrm{U}$ and ${ }^{206} \mathrm{~Pb} /{ }^{207} \mathrm{~Pb}$ ages) or $>5 \%$ reverse discordant are not considered further. The resulting interpreted ages are shown on $\mathrm{Pb} / \mathrm{U}$ concordia diagrams and relative age-probability diagrams using the routines in Isotope/Ex, rev.3.0 (Ludwig, 2003). The age-probability diagrams show each age and its uncertainty (for measurement error only) as a normal distribution, and sum all ages from a sample into a single probability density function. All the data and GPS positions are shown in the Supplementary Table DR1.

The age uncertainty range of the Liuqu Formation (Gongjiongla and $\mathrm{Za}$ localities) is indicated by $\mathrm{U}-\mathrm{Pb}$ ages of detrital zircons of 54-58 Ma (Fig. DR2). A tuffite sample from Xialu section has determined a concordia age of 59.3 \pm 1.6 Ma by the 9 youngest zircons with age from 56.4 Ma to 61.4 Ma (Fig. DR3). Together with the megafossil age estimations (Fang et al., 2005, 2006), we therefore assign the minimum age of the Liuqu paleofloras at $56 \mathrm{Ma}$ based on the youngest zircon ages.

The youngest $\mathrm{U}-\mathrm{Pb}$ agesof detrital zircons of the Qiuwu Formation (Qiabulin locality) is 26-21 Ma. These ages are also consistent with the Qiuwu Formation at Angren in the west and the equivalent unit of the Luobusha Formation at Zedong in the east (Li et al., 2015). The age of the overlying Dazuqu Formation is assigned to be no older than $\sim 19 \mathrm{Ma}$ at Qiabulin locality (Fig. DR2), which is determined by $\mathrm{U}-\mathrm{Pb}$ ages of detrital zircons and ${ }^{40} \mathrm{Ar} /{ }^{39} \mathrm{Ar}$ analyses of laterally equivalent tuffs (Aitchison et al., 2009). So the age of the Qiuwu Formation is constrained to be 21-19 Ma. The 
Namling assemblage (Wulong Formation) was dated on ${ }^{40} \mathrm{Ar} /{ }^{39} \mathrm{Ar}$ analysis of bounding volcanic rocks, at $\sim 15 \mathrm{Ma}$ (Spicer et al., 2003).

\section{S3. TABLES}

Table DR1 U-Pb isotopic analysis of detrital zircons from Liuqu, Qiuwu and Dazuqu Formations.

Table DR2. Major elements data for minerals comprising the tuffite by electron probe microanalysis.

Table DR3 CLAMP scores describing woody dicot leaf form within the Liuqu, Qiuwu, Namling and India paleofloras.

Table DR4 Climatic parameters predicted by CLAMP and their associated statistical uncertainties. Modern climate data of Shillong (1966-1978) and Lhasa (1986-1992) (http://www.iaea.org/water) are from local observation stations. Reconstructed climate parameters for each of the localities are mean annual temperature (MAT), cold month mean temperature (CMMT), warm month mean temperature (WMMT), length of the growing season (LGS), growing season precipitation (GSP), mean monthly growing season precipitation (MMGSP), precipitation during the three consecutive wettest months (3-WET) and three consecutive driest months (3-DRY), specific humidity $(\mathrm{SH})$, relative humidity $(\mathrm{RH})$ and enthalpy (ENTHAL).

Table DR5 Paleoelevation estimated by CLAMP. 


\section{S4. FIGURES}

Figure DR1. Representative leaf fossils from the Liuqu Formation, Gongjiongla and Za localities. Scale bars $=2 \mathrm{~cm}$.

Figure DR2. Mean age diagram for a tuffite (sample 2014T171) from the lower part of the Liuqu Formation. A. Field photo for the sampling site. Hammer for scale. B. Electron probe microanalyzer (EPMA) backscatter scanning image for the sample, which mainly contains the volcanic glass and hydrated clay minerals. C. Concordia plots for the 9 youngest zircon ages and $\mathbf{D}$. U/Th ratios implicating magmatic zircons.

Figure DR3. Zircon U-Pb age probability density diagram for the Liuqu, Qiuwu and Dazuqu formations. The youngest age populations constrain the maximum deposition age of the formations.

Figure DR4. Diorite clasts from Liuqu conglomerate and zircon age diagram. A. Field photograph for conglomerates from the base of the Liuqu Formation and the diorite clasts. B. Age probability diagram for the diorite clasts.

Figure DR5. Representative leaf fossils from the Qiuwu Formation, Qiabulin locality. Scale bars $=2 \mathrm{~cm}$.

Figure DR6. Field photographs. A. Liuqu Formation at Za locality. B. Qiuwu and Dazuqu Formation at Qiabulin locality. C. Palm trunk (arrowed) in the Liuqu Formation. D. Part of a palm leaf (arrowed) from Liuqu Formation. E. Base of a palm leaf (arrowed) from Liuqu Formation.

Figure DR7. Annual average moist enthalpy at mean sea level. A. Bristol Research Initiative for the Dynamic Global Environment (BRIDGE) modeling results for annual 
average moist enthalpy at mean sea level in middle Miocene (10 Ma) time. Run xakfl using a slab (50m) ocean with dynamic heat transfer and $395 \mathrm{ppm} \mathrm{CO}_{2}, 449 \mathrm{ppbv} \mathrm{CH}_{4}$ and 287 ppbv $\mathrm{N}_{2} \mathrm{O}$, and present day orbital configuration. B. BRIDGE modeling results for annual average moist enthalpy at mean sea level in early Eocene (55 Ma) time. Run xakfg using a slab (50m) ocean with dynamic heat transfer and 1378 ppmv $\mathrm{CO}_{2}, 790$ ppbv $\mathrm{CH}_{4}$ and 284 ppbv $\mathrm{N}_{2} \mathrm{O}$ and present day orbital configuration. The dashed lines show the likely extents of greater India and greater Asia respectively during the Early Eocene (Ding et al., 2005).

\section{REFERENCES CITED}

Aitchison, J.C., Xia, X.P., Baxter, A.T., and Ali. J.R., 2011, Detrital zircon U-Pb ages along the Yarlung-Tsangpo suture zone, Tibet: Implications for oblique convergence and collision between India and Asia: Gondwana Research, v. 20, p. 691-709, doi:10.1016/j.gr.2011.04.002.

Andersen, T., 2002, Correction of common lead in U-Pb analyses that do not report Pb-204: Chemical Geology, v.192, p.59-79.

Davis, A.M., Aitchison, J.C., Bai, D.Z., Luo, H., and Zyabrev, S., 2002, Paleogene island arc collision-related conglomerates, Yarlung-Tsangpo suture zone, Tibet: Sedimentary Geology, v.150, p.247-273, doi:10.1016/S0037-0738(01)00199-3.

DeCelles, P. G., Kapp, P., Gehrels, G. E., and Ding, L., 2014, Paleocene-Eocene foreland basin evolution in the Himalaya of southern Tibet and Nepal: 
Implications for the age of initial India-Asia collision: Tectonics, v.33, p. 824-849, doi:10.1002/2014TC003522.

Fang, A.M., Yan, Z., Liu, X.H., Pan, Y.S., Li, J.L., Yu, L.J., Huang, F.X., and Tao, J.R., 2006, The age of the plant fossil assemblage in the Liuqu Conglomerate of southern Tibet and its tectonic significance, Progress of Nature Science, v.16, p.55-64.

Ji, W.Q., Wu, F.Y., Chung, S.L., Li, J.X., and Liu, C.Z., 2009, Zircon U-Pb geochronology and Hf isotopic constraints on petrogenesis of the Gangdese batholith, southern Tibet: Chemical Geology, v.262, p.229-245, doi:10.1016/j.chemgeo.2009.01.020.

Li, S., Ding, L., Xu, Q., Wang, H., Yue, Y., and Baral, U., 2015, The evolution of Yarlung Tsangpo River: Constraints from the age and provenance of the Gangdese Conglomerates, southern Tibet: Gondwana Research, doi: 10.1016/j.gr.2015.05.010.

Ludwig, K.R., 2003, Isotope/Ex, rev.3.0, Berkeley Geochronology Center, Special Publication 1a, 56pp.

Najman, Y., Appel, E., Boudagher-Fadel, M., Bown, P., Carter, A., Garzanti, E., Godin, L., Han, J.T., Liebke, U., Oliver, G., Parrish, R., and Vezzoli, G., 2010, Timing of India-Asia collision: Geological, biostratigraphic, and palaeomagnetic constraints: Journal of Geophysics Research-Solid Earth, v. 115( B12416), p. 1-18, doi: 10.1029/2010JB007673.

Sláma, J., Košler, J., Condon, D.J., Crowley, J.L., Gerdes, A., Hanchar, J.M., 
Horstwood, M.S., Morris, G.A., Nasdala, L., and Norberg, N., 2008, Plesovice zircon - A new natural reference material for $\mathrm{U}-\mathrm{Pb}$ and $\mathrm{Hf}$ isotopic microanalysis:

Chemical Geology, v.249, p.1-35, doi:10.1016/j.chemgeo.2007.11.005. 


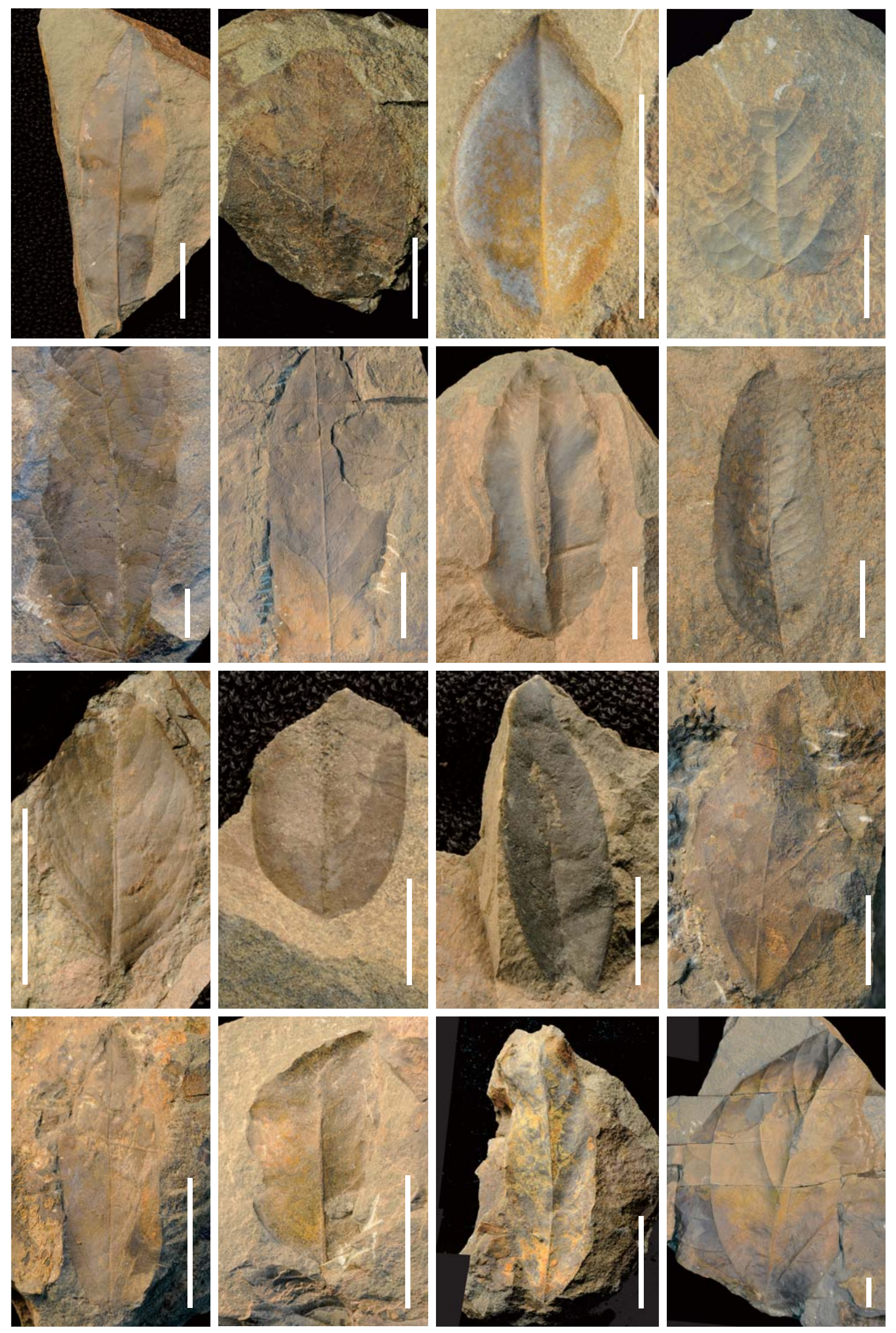



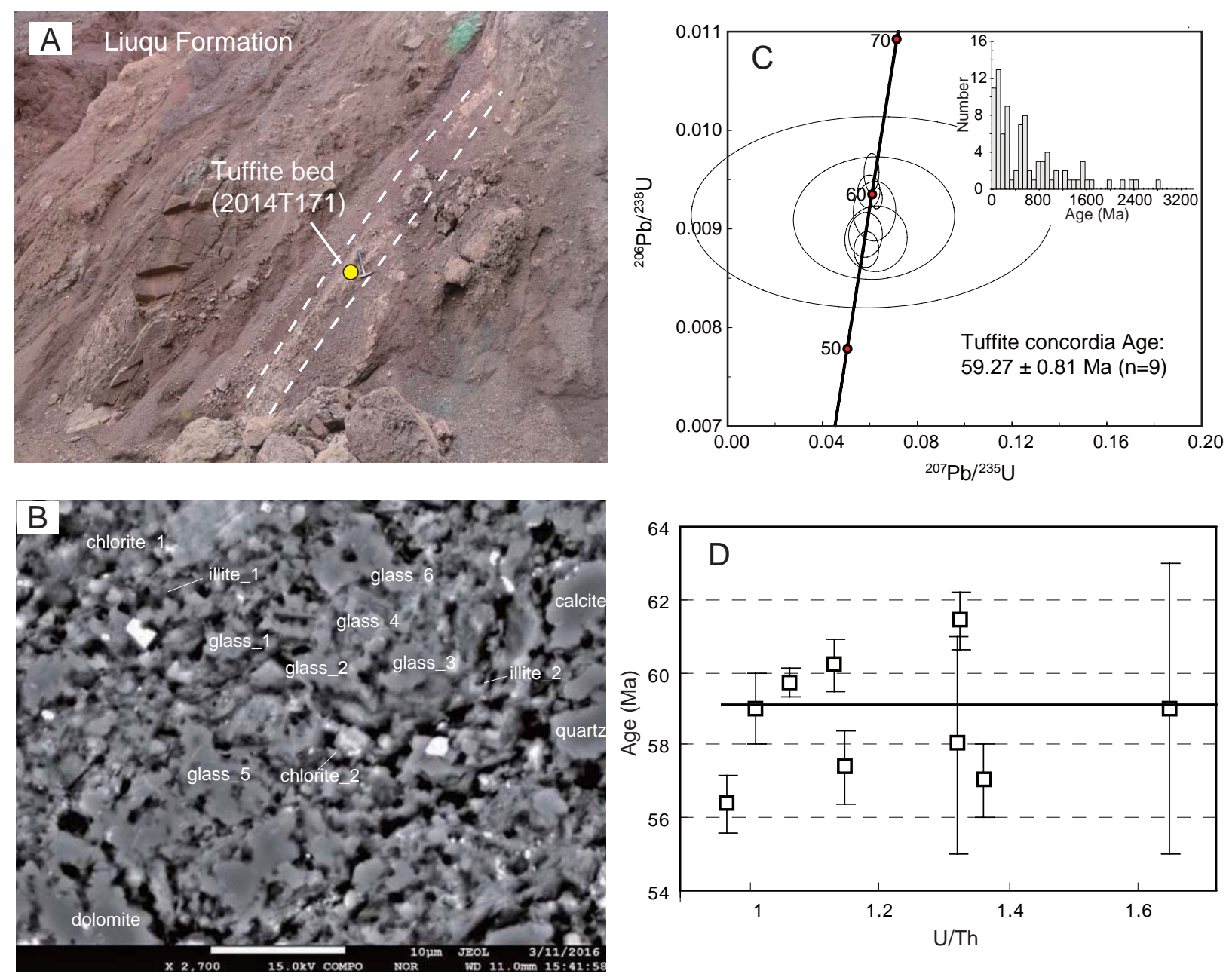


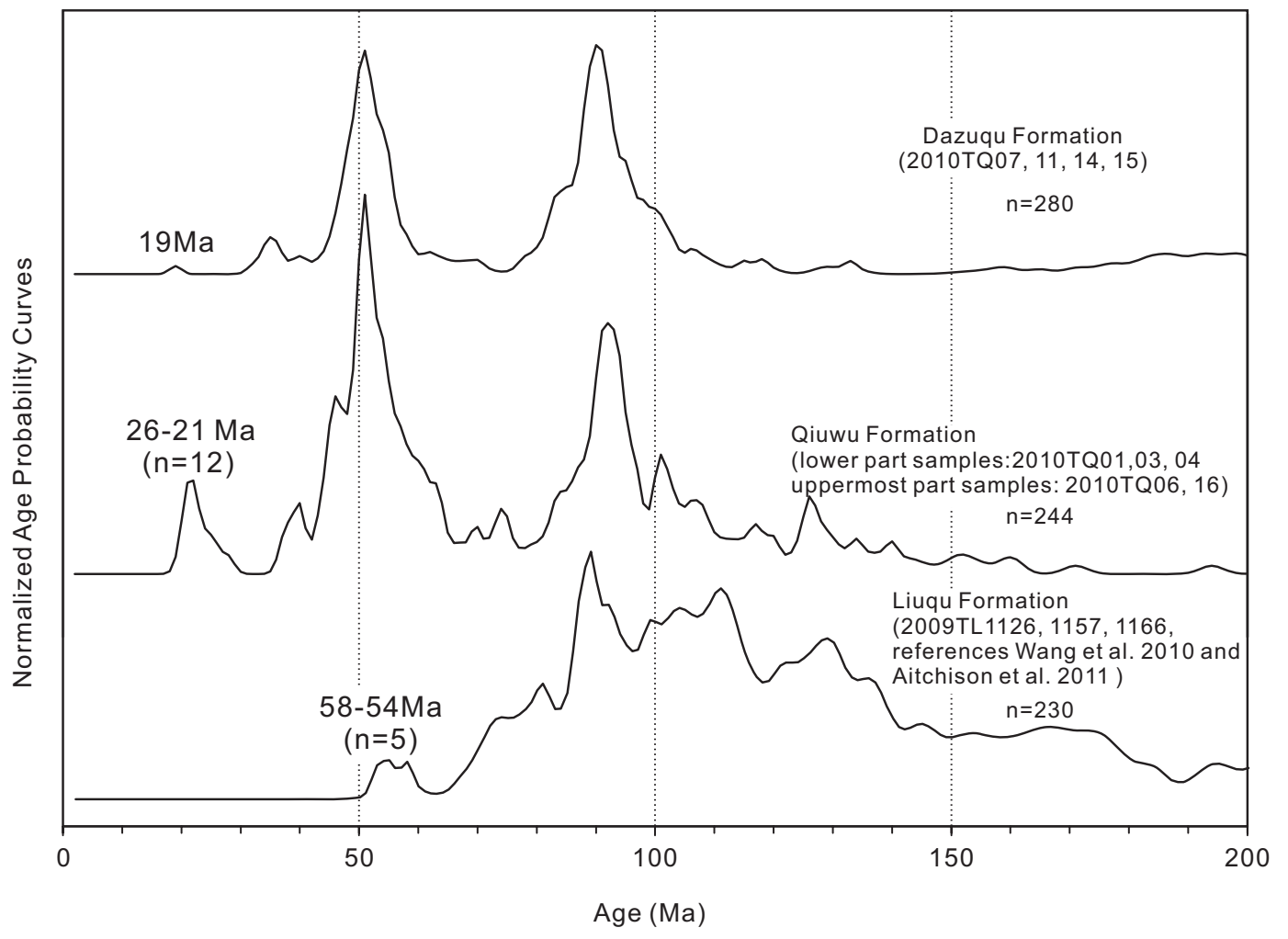



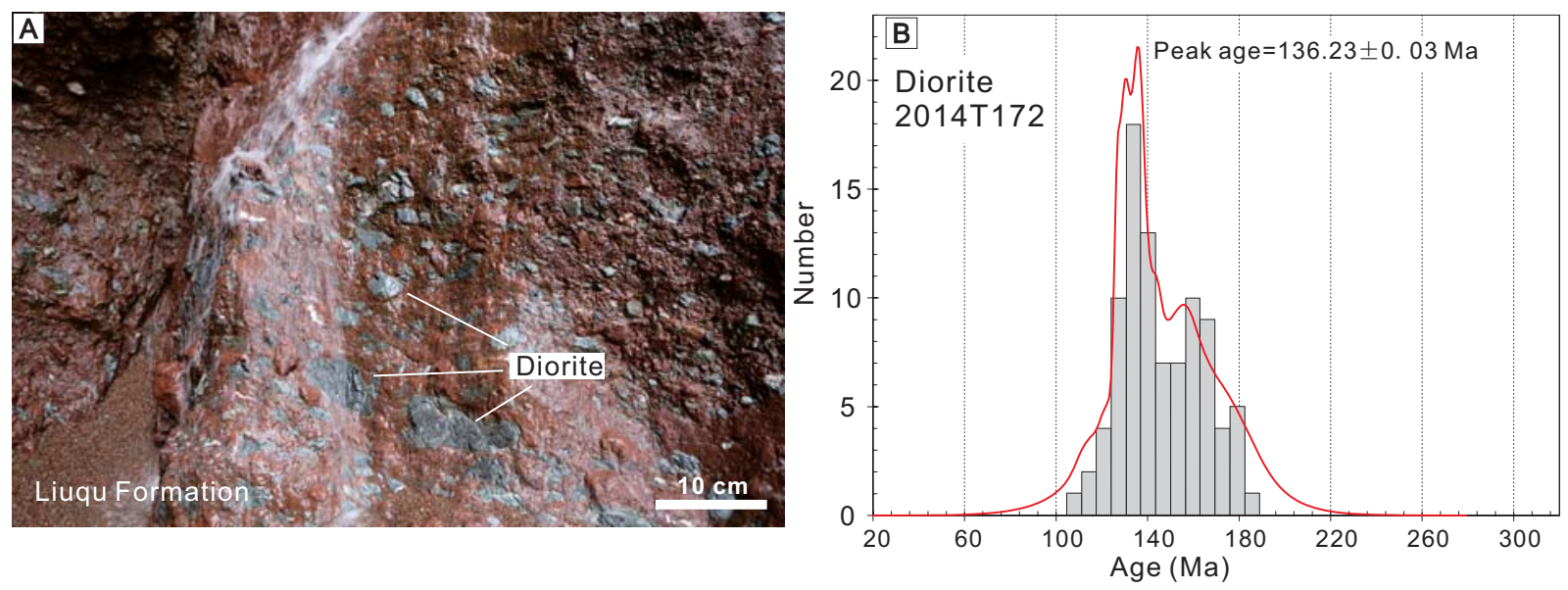


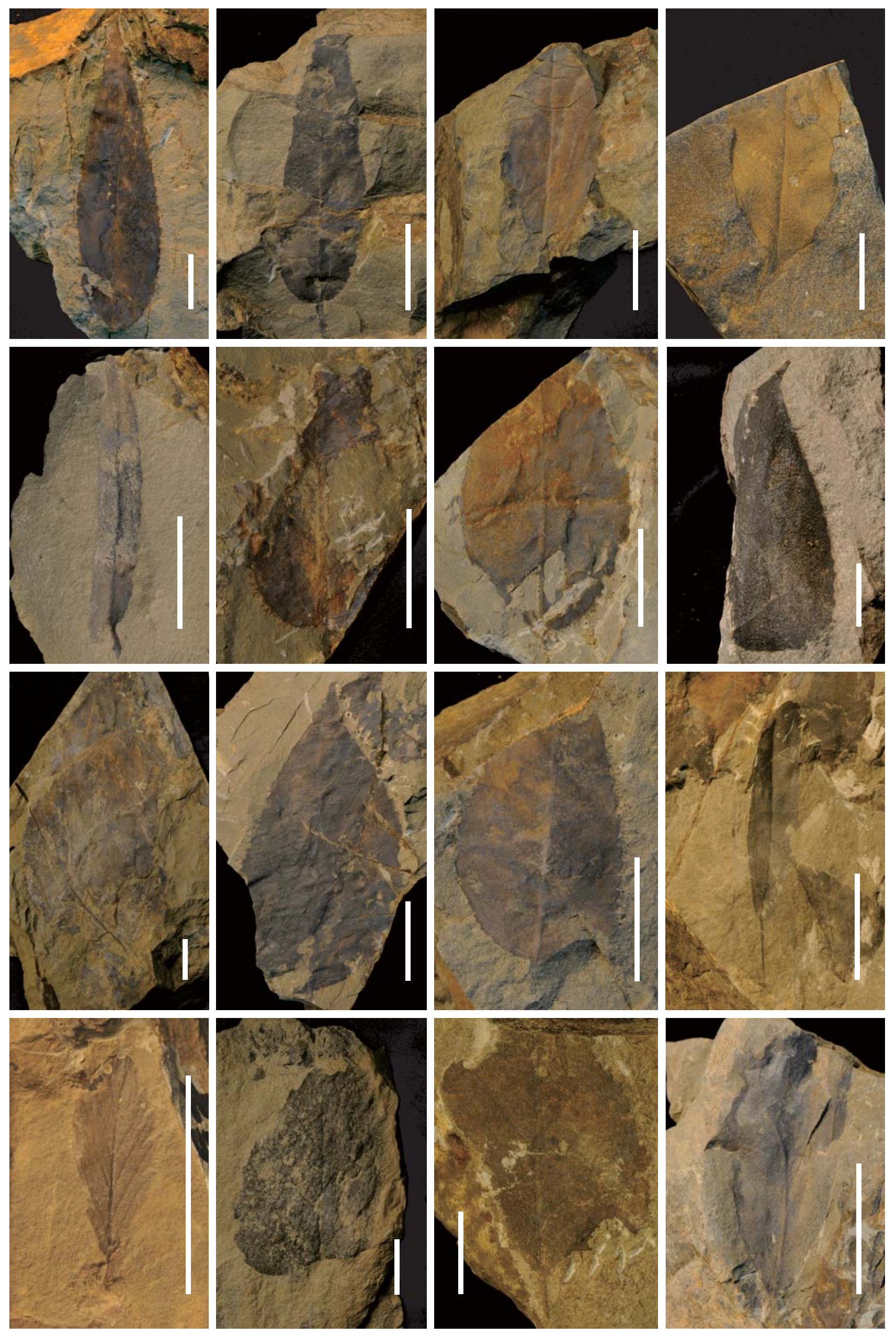



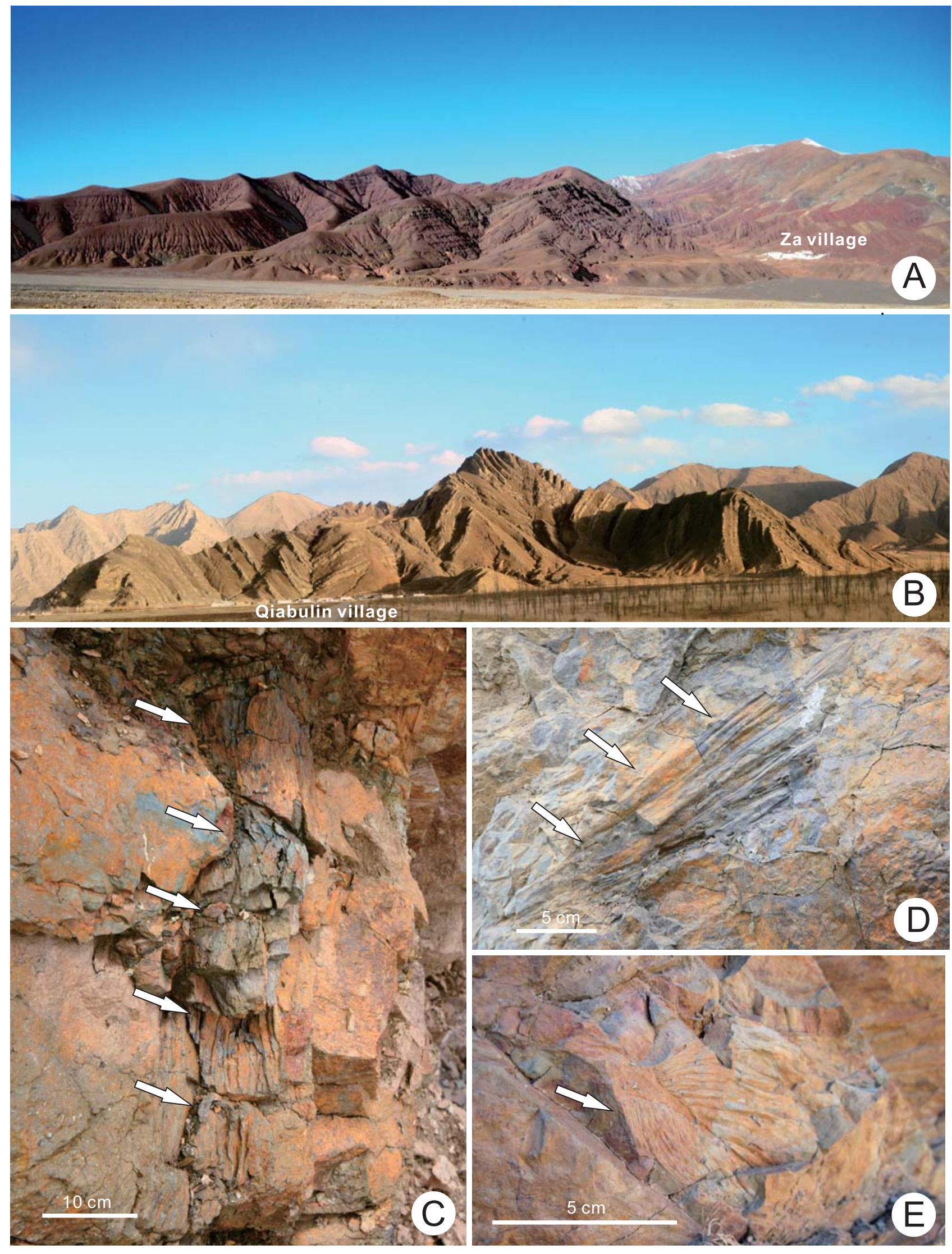

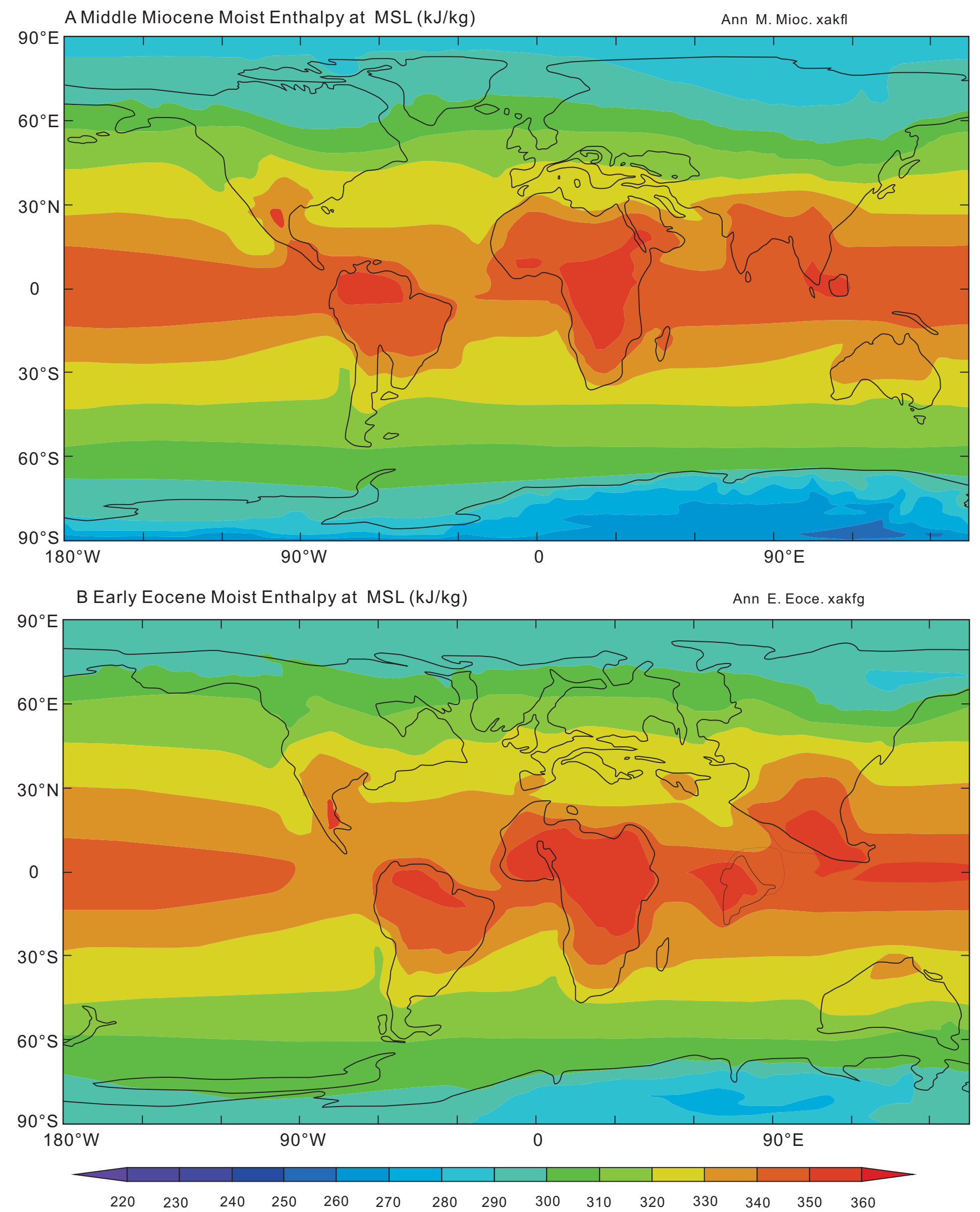
TABLE DR1. U-Pb ISOTOPIC ANALYSIS OF TUFFITE AND DETRITAL ZIRCONS FROM LIUQU, QIUWU AND DAZUQU FORMATIONS.

\begin{tabular}{|c|c|c|c|c|c|c|c|c|c|c|c|c|c|c|c|c|c|c|c|}
\hline \multirow[t]{2}{*}{ Analysis } & \multicolumn{9}{|c|}{ Isotopic ratios } & \multicolumn{10}{|c|}{ Isotopic ages (Ma) } \\
\hline & $\begin{array}{c}{ }^{207} \mathrm{~Pb} / \\
{ }^{206} \mathrm{~Pb}\end{array}$ & $1 \sigma$ & $\begin{array}{c}{ }^{207} \mathrm{~Pb} / \\
{ }^{235} \mathrm{U}\end{array}$ & $1 \sigma$ & $\begin{array}{c}{ }^{206} \mathrm{~Pb} / \\
{ }^{238} \mathrm{U}\end{array}$ & $1 \sigma$ & $\begin{array}{l}{ }^{208} \mathrm{~Pb} / \\
{ }^{232} \mathrm{Th}\end{array}$ & $1 \sigma$ & ${ }^{238} \mathrm{U} /$ & $\begin{array}{l}{ }^{207} \mathrm{~Pb} / \\
{ }^{206} \mathrm{~Pb}\end{array}$ & $1 \sigma$ & ${ }^{207} \mathrm{~Pb} /$ & $1 \sigma$ & ${ }^{206} \mathrm{~Pb} /$ & $1 \sigma$ & $\begin{array}{l}{ }^{208} \mathrm{~Pb} / \\
{ }^{232} \mathrm{Th}\end{array}$ & $1 \sigma$ & $\begin{array}{l}\text { Used } \\
\text { age }\end{array}$ & $2 \sigma$ \\
\hline \multicolumn{20}{|c|}{2014 T171 (Liuqu tuffite, Xialu section, $n=103, \mathrm{~N}^{\circ} 9^{\circ} 06.15^{\prime} \quad \mathrm{E88}^{\circ} 58.10^{\prime}, 4148 \mathrm{~m}$ ) } \\
\hline 2014T171-097 & 0.0483 & 0.00294 & 0.05854 & .0035 & 0.0088 & 0.00012 & 0.0029 & 0.00007 & 0.96 & 115 & 107 & 58 & 3 & 56.4 & 0.8 & 58 & 1 & 56.4 & 1.6 \\
\hline 2014T171-021 & 0.051 & 0.00721 & 0.06263 & 0.0087 & 0.0089 & 0.00022 & 0.0029 & 0.00016 & 1.36 & 242 & 264 & 62 & 8 & 57 & 1 & 58 & 3 & 57 & 2 \\
\hline 2014T171-100 & 0.0473 & 0.00391 & 0.05818 & 0.0047 & 0.0089 & 0.00015 & 0.0025 & 0.00008 & 1.14 & 62 & 147 & 57 & 5 & 57.4 & 1 & 50 & 2 & 57.4 & 2 \\
\hline 2014T171-067 & 0.0492 & 0.01802 & 0.0618 & 0.0225 & 0.0091 & 0.00041 & 0.003 & 0.0003 & 1.32 & 159 & 551 & 61 & 21 & 58 & 3 & 60 & 6 & 58 & 6 \\
\hline 2014T171-040 & 0.0483 & 0.04 & 0.06099 & 0.0504 & 0.0092 & 0.00064 & 0.0051 & 0.00077 & 1.65 & 112 & 1131 & 60 & 48 & 59 & 4 & 102 & 15 & 59 & 8 \\
\hline 2014T171-101 & 0.0491 & 0.0048 & 0.06198 & 0.0059 & 0.0092 & 0.0002 & 0.0027 & 0.00011 & 1.01 & 150 & 173 & 61 & 6 & 59 & 1 & 54 & 2 & 59 & 2 \\
\hline 2014T171-102 & 0.0491 & 0.00132 & 0.06293 & 0.0016 & 0.0093 & 0.00007 & 0.0029 & 0.00003 & 1.06 & 153 & 45 & 62 & 2 & 59.7 & 0.4 & 59.3 & 0.6 & 59.7 & 0.8 \\
\hline 2014T171-098 & 0.0461 & 0.00246 & 0.05958 & 0.0031 & 0.0094 & 0.00011 & 0.0031 & 0.00009 & 1.13 & & 116 & 59 & 3 & 60.2 & 0.7 & 62 & 2 & 60.2 & 1.4 \\
\hline 2014T171-017 & 0.0461 & 0.00171 & 0.06075 & 0.0021 & 0.0096 & 0.00013 & 0.0037 & 0.00035 & 1.33 & & 78 & 60 & 2 & 61.4 & 0.8 & 76 & 7 & 61.4 & 1.6 \\
\hline 2014T171-069 & 0.0472 & 0.01927 & 0.06963 & 0.0283 & 0.0107 & 0.00029 & 0.0027 & 0.00048 & 1.44 & 61 & 633 & 68 & 27 & 69 & 2 & 54 & 10 & 69 & 4 \\
\hline $171-013$ & 0.0503 & 0.00764 & 0.07758 & 0.0117 & 0.0112 & 0.00022 & 0.004 & 0.00018 & 1.36 & 208 & 284 & 76 & 11 & 72 & 1 & 80 & 4 & 72 & 2 \\
\hline 2014T171-073 & 0.0498 & 0.01309 & 0.09604 & 0.0252 & 0.014 & 0.00027 & 0.004 & 0.00039 & 1.17 & 184 & 433 & 93 & 23 & 90 & 2 & 81 & 8 & 90 & 4 \\
\hline 2014T171-027 & 0.0461 & 0.00381 & 0.09185 & 0.0075 & 0.0145 & 0.00023 & 0.0047 & 0.00019 & 1.06 & & 182 & 89 & 7 & 93 & 1 & 96 & 4 & 93 & 2 \\
\hline 2014T171-086 & 0.0486 & 0.01209 & 0.0972 & 0.0241 & 0.0145 & 0.00032 & 0.005 & 0.0003 & 1.08 & 129 & 399 & 94 & 22 & 93 & 2 & 100 & 6 & 3 & 4 \\
\hline 2014T171-025 & 0.0485 & 0.01269 & 0.09981 & 0.0259 & 0.0149 & 0.00052 & 0.0052 & 0.00045 & 1.54 & 123 & 398 & 97 & 24 & 96 & 3 & 105 & 9 & 96 & 6 \\
\hline 2014T171-063 & 0.0482 & 0.00585 & 0.10816 & 0.013 & 0.0163 & 0.00025 & 0.0064 & 0.00029 & 1.41 & 109 & 236 & 104 & 12 & 104 & 2 & 128 & 6 & 104 & 4 \\
\hline 2014T171-093 & 0.0506 & 0.01347 & 0.11572 & 0.0307 & 0.0166 & 0.00041 & 0.005 & 0.00052 & 0.90 & 223 & 439 & 111 & 28 & 106 & 3 & 102 & 10 & 106 & 6 \\
\hline 2014T171-060 & 0.0522 & 0.02185 & 0.12734 & 0.0532 & 0.0177 & 0.00058 & 0.0146 & 0.00062 & 1.01 & 293 & 694 & 122 & 48 & 113 & 4 & 294 & 12 & 113 & 8 \\
\hline 2014T171-019 & 0.0518 & 0.00417 & 0.13582 & 0.0108 & 0.019 & 0.00027 & 0.0065 & 0.00015 & 0.97 & 274 & 154 & 129 & 10 & 122 & 2 & 131 & 3 & 122 & 4 \\
\hline 2014T171-068 & 0.0468 & 0.00403 & 0.12362 & 0.0105 & 0.0192 & 0.0002 & 0.0048 & 0.00017 & 1.28 & 40 & 169 & 118 & 10 & 122 & 1 & 98 & 3 & 122 & 2 \\
\hline 2014T171-092 & 0.0485 & 0.06139 & 0.12881 & 0.1626 & 0.0193 & 0.00183 & 0.0061 & 0.00226 & 0.89 & 124 & 1460 & 123 & 146 & 123 & 12 & 123 & 45 & 123 & 24 \\
\hline 2014T171-046 & 0.0503 & 0.00921 & 0.13857 & 0.0253 & 0.02 & 0.00034 & 0.0068 & 0.00029 & 0.88 & 209 & 325 & 132 & 23 & 128 & 2 & 138 & 6 & 128 & 4 \\
\hline 2014T171-089 & 0.0505 & 0.00498 & 0.14617 & 0.0143 & 0.021 & 0.00023 & 0.0064 & 0.00012 & 0.47 & 218 & 201 & 139 & 13 & 134 & 1 & 129 & 2 & 134 & 2 \\
\hline 2014T171-088 & 0.0469 & 0.02327 & 0.13919 & 0.0689 & 0.0215 & 0.00071 & 0.0019 & 0.00083 & 0.96 & 45 & 792 & 132 & 61 & 137 & 4 & 38 & 17 & 137 & 8 \\
\hline 2014T171-079 & 0.049 & 0.0106 & 0.1673 & 0.0361 & 0.0248 & 0.00038 & 0.0128 & 0.00042 & 0.99 & 148 & 363 & 157 & 31 & 158 & 2 & 258 & 8 & 158 & 4 \\
\hline 2014T171-043 & 0.0482 & 0.00757 & 0.16838 & 0.0263 & 0.0253 & 0.00035 & 0.0038 & 0.00061 & 1.71 & 111 & 287 & 158 & 23 & 161 & 2 & 76 & 12 & 161 & 4 \\
\hline
\end{tabular}




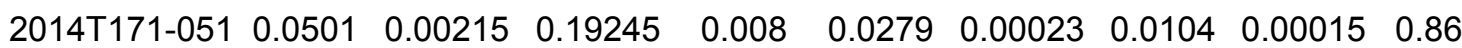
$\begin{array}{llllllllll}2014 T 171-002 & 0.0537 & 0.00171 & 0.22171 & 0.0067 & 0.03 & 0.00025 & 0.0087 & 0.00012 & 1.34\end{array}$ $\begin{array}{lllllllllll}2014 \mathrm{~T} 171-099 & 0.0487 & 0.00206 & 0.21144 & 0.0087 & 0.0315 & 0.00025 & 0.0051 & 0.00025 & 2.23\end{array}$ $\begin{array}{lllllllllll}2014 T 171-028 & 0.05 & 0.00137 & 0.22018 & 0.0056 & 0.0319 & 0.00024 & 0.0097 & 0.00012 & 1.57\end{array}$ $\begin{array}{llllllllll}2014 \mathrm{~T} 171-031 & 0.0546 & 0.0018 & 0.26767 & 0.0086 & 0.0355 & 0.00028 & 0.0111 & 0.00007 & 2.72\end{array}$ $\begin{array}{lllllllllll}2014 T 171-059 & 0.0499 & 0.00285 & 0.24528 & 0.0138 & 0.0357 & 0.00031 & 0.0194 & 0.00084 & 3.96\end{array}$ $\begin{array}{llllllllll}2014 \mathrm{~T} 171-015 & 0.05 & 0.00216 & 0.25426 & 0.0106 & 0.0369 & 0.00035 & 0.0124 & 0.00032 & 2.94\end{array}$ $\begin{array}{llllllllll}2014 T 171-053 & 0.0505 & 0.00297 & 0.25709 & 0.0149 & 0.037 & 0.00034 & 0.0077 & 0.00033 & 1.68\end{array}$ $\begin{array}{llllllllll}2014 T 171-080 & 0.0536 & 0.00217 & 0.29775 & 0.0117 & 0.0403 & 0.00029 & 0.0023 & 0.00031 & 2.09\end{array}$ $\begin{array}{lllllllllll}2014 T 171-039 & 0.0507 & 0.00604 & 0.29415 & 0.0347 & 0.0421 & 0.00065 & 0.009 & 0.00046 & 0.89\end{array}$ $\begin{array}{llllllllll}2014 T 171-083 & 0.052 & 0.02616 & 0.3198 & 0.1603 & 0.0446 & 0.00175 & 0.0039 & 0.00284 & 1.09\end{array}$ $\begin{array}{lllllllllll}2014 \mathrm{~T} 171-096 & 0.0519 & 0.00317 & 0.32229 & 0.0194 & 0.0451 & 0.00046 & 0.0121 & 0.00036 & 1.59\end{array}$ $\begin{array}{llllllllll}2014 T 171-081 & 0.0505 & 0.00739 & 0.32441 & 0.047 & 0.0466 & 0.0012 & 0.0129 & 0.00101 & 2.19\end{array}$ $\begin{array}{llllllllll}2014 T 171-065 & 0.053 & 0.00433 & 0.39797 & 0.0322 & 0.0545 & 0.00057 & 0.0062 & 0.00103 & 2.39\end{array}$ $\begin{array}{llllllllll}2014 T 171-049 & 0.0545 & 0.00312 & 0.47329 & 0.0267 & 0.0631 & 0.00056 & 0.0106 & 0.00091 & 2.12\end{array}$ $\begin{array}{llllllllll}2014 T 171-094 & 0.0586 & 0.00152 & 0.56789 & 0.0137 & 0.0703 & 0.00055 & 0.0219 & 0.00031 & 1.83\end{array}$ $\begin{array}{llllllllll}2014 T 171-010 & 0.0627 & 0.00339 & 0.66492 & 0.0349 & 0.0769 & 0.00106 & 0.0205 & 0.00056 & 1.29\end{array}$ $\begin{array}{lllllllllll}2014 \mathrm{~T} 171-006 & 0.0604 & 0.0025 & 0.65602 & 0.0262 & 0.0789 & 0.00084 & 0.0256 & 0.00037 & 0.82\end{array}$ $\begin{array}{llllllllll}2014 T 171-036 & 0.0579 & 0.00884 & 0.63273 & 0.0961 & 0.0793 & 0.00137 & 0.0384 & 0.00185 & 1.74\end{array}$ $\begin{array}{llllllllll}2014 T 171-072 & 0.0563 & 0.00344 & 0.63321 & 0.038 & 0.0816 & 0.00089 & 0.022 & 0.00029 & 0.52\end{array}$ $\begin{array}{lllllllllll}2014 T 171-004 & 0.0645 & 0.00165 & 0.73669 & 0.0174 & 0.0829 & 0.00067 & 0.0276 & 0.00029 & 0.95\end{array}$ $\begin{array}{lllllllllll}2014 T 171-056 & 0.0598 & 0.0045 & 0.68412 & 0.0509 & 0.083 & 0.00091 & 0.0005 & 0.00182 & 2.75\end{array}$ $\begin{array}{llllllllll}2014 T 171-034 & 0.0593 & 0.00236 & 0.67925 & 0.026 & 0.0831 & 0.00085 & 0.0254 & 0.00059 & 1.96\end{array}$ $\begin{array}{lllllllllll}2014 \mathrm{~T} 171-085 & 0.056 & 0.0018 & 0.665 & 0.0205 & 0.0862 & 0.00064 & 0.0151 & 0.00035 & 1.38\end{array}$ $\begin{array}{lllllllllll}2014 T 171-018 & 0.0575 & 0.00166 & 0.69136 & 0.0187 & 0.0873 & 0.00072 & 0.0283 & 0.00035 & 1.16\end{array}$ $\begin{array}{llllllllll}2014 \mathrm{~T} 171-047 & 0.0607 & 0.01994 & 0.74197 & 0.2422 & 0.0887 & 0.00416 & 0.0254 & 0.00185 & 0.70\end{array}$ $\begin{array}{llllllllll}2014 T 171-090 & 0.0588 & 0.00621 & 0.72062 & 0.0754 & 0.0889 & 0.00138 & 0.0165 & 0.00191 & 2.97\end{array}$ $\begin{array}{llllllllll}2014 T 171-003 & 0.0634 & 0.00149 & 0.77889 & 0.0168 & 0.0891 & 0.00068 & 0.0281 & 0.00036 & 1.67\end{array}$ $\begin{array}{llllllllll}2014 T 171-084 & 0.0614 & 0.02816 & 0.75931 & 0.347 & 0.0897 & 0.00479 & 0.0206 & 0.00241 & 0.73\end{array}$ $\begin{array}{llllllllll}2014 T 171-042 & 0.0591 & 0.00263 & 0.77187 & 0.0335 & 0.0948 & 0.00093 & 0.0331 & 0.00182 & 4.64\end{array}$ $\begin{array}{llllllllll}2014 T 171-050 & 0.0618 & 0.00414 & 0.81109 & 0.0534 & 0.0952 & 0.0012 & 0.0235 & 0.00058 & 0.64\end{array}$ $\begin{array}{llllllllll}2014 \mathrm{~T} 171-091 & 0.0608 & 0.00365 & 0.89917 & 0.0531 & 0.1074 & 0.00122 & 0.0241 & 0.00265 & 1.81\end{array}$ $\begin{array}{llllllllllll}2014 T 171-082 & 0.0653 & 0.00602 & 0.98603 & 0.0899 & 0.1096 & 0.0019 & 0.0476 & 0.00426 & 4.16\end{array}$ $\begin{array}{llllllllll}2014 T 171-037 & 0.0608 & 0.00167 & 0.94773 & 0.0246 & 0.1131 & 0.00095 & 0.052 & 0.00219 & 7.86\end{array}$

$\begin{array}{cccccccccc}201 & 82 & 179 & 7 & 177 & 1 & 209 & 3 & 177 & 2 \\ 356 & 53 & 203 & 6 & 190 & 2 & 175 & 2 & 190 & 4 \\ 134 & 80 & 195 & 7 & 200 & 2 & 104 & 5 & 200 & 4 \\ 195 & 45 & 202 & 5 & 203 & 1 & 195 & 2 & 203 & 2 \\ 397 & 76 & 241 & 7 & 225 & 2 & 223 & 1 & 225 & 4 \\ 190 & 112 & 223 & 11 & 226 & 2 & 388 & 17 & 226 & 4 \\ 194 & 80 & 230 & 9 & 234 & 2 & 249 & 6 & 234 & 4 \\ 217 & 116 & 232 & 12 & 234 & 2 & 154 & 7 & 234 & 4 \\ 355 & 76 & 265 & 9 & 255 & 2 & 46 & 6 & 255 & 4 \\ 229 & 239 & 262 & 27 & 266 & 4 & 182 & 9 & 266 & 8 \\ 287 & 848 & 282 & 123 & 281 & 11 & 79 & 57 & 281 & 22 \\ 279 & 120 & 284 & 15 & 284 & 3 & 244 & 7 & 284 & 6 \\ 216 & 272 & 285 & 36 & 294 & 7 & 259 & 20 & 294 & 14 \\ 328 & 166 & 340 & 23 & 342 & 3 & 125 & 21 & 342 & 6 \\ 390 & 112 & 393 & 18 & 394 & 3 & 213 & 18 & 394 & 6 \\ 552 & 39 & 457 & 9 & 438 & 3 & 438 & 6 & 438 & 6 \\ 699 & 89 & 518 & 21 & 477 & 6 & 410 & 11 & 477 & 12 \\ 616 & 68 & 512 & 16 & 489 & 5 & 512 & 7 & 489 & 10 \\ 527 & 311 & 498 & 60 & 492 & 8 & 761 & 36 & 492 & 16 \\ 465 & 115 & 498 & 24 & 505 & 5 & 440 & 6 & 505 & 10 \\ 758 & 36 & 560 & 10 & 513 & 4 & 550 & 6 & 513 & 8 \\ 596 & 144 & 529 & 31 & 514 & 5 & 11 & 37 & 514 & 10 \\ 577 & 65 & 526 & 16 & 515 & 5 & 508 & 12 & 515 & 10 \\ 452 & 55 & 518 & 12 & 533 & 4 & 303 & 7 & 533 & 8 \\ 510 & 45 & 534 & 11 & 539 & 4 & 563 & 7 & 539 & 8 \\ 629 & 563 & 564 & 141 & 548 & 25 & 507 & 36 & 548 & 50 \\ 560 & 205 & 551 & 45 & 549 & 8 & 331 & 38 & 549 & 16 \\ 722 & 33 & 585 & 10 & 550 & 4 & 560 & 7 & 550 & 8 \\ 654 & 811 & 574 & 200 & 554 & 28 & 411 & 48 & 554 & 56 \\ 571 & 78 & 581 & 19 & 584 & 5 & 658 & 36 & 584 & 10 \\ 669 & 120 & 603 & 30 & 586 & 7 & 469 & 11 & 586 & 14 \\ 630 & 108 & 651 & 28 & 657 & 7 & 481 & 52 & 657 & 14 \\ 783 & 165 & 697 & 46 & 670 & 11 & 940 & 82 & 670 & 22 \\ 632 & 41 & 677 & 13 & 691 & 6 & 1024 & 42 & 691 & 12\end{array}$




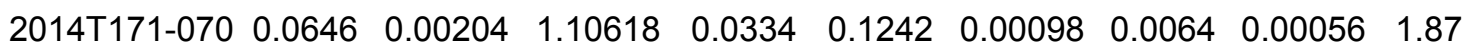
$\begin{array}{llllllllll}2014 T 171-078 & 0.0714 & 0.00263 & 1.25919 & 0.0448 & 0.1279 & 0.00121 & 0.0214 & 0.0007 & 1.38\end{array}$ $\begin{array}{llllllllll}2014 T 171-022 & 0.0673 & 0.0013 & 1.22362 & 0.0223 & 0.132 & 0.00089 & 0.0402 & 0.00024 & 4.70\end{array}$ $\begin{array}{llllllllll}2014 T 171-041 & 0.0656 & 0.00391 & 1.24186 & 0.0727 & 0.1375 & 0.0019 & 0.009 & 0.00144 & 1.53\end{array}$ $\begin{array}{llllllllll}2014 \mathrm{~T} 171-023 & 0.0707 & 0.00116 & 1.37514 & 0.0187 & 0.141 & 0.0009 & 0.0378 & 0.00092 & 10.23\end{array}$ $\begin{array}{llllllllll}2014 T 171-048 & 0.0687 & 0.00194 & 1.36753 & 0.0367 & 0.1444 & 0.00124 & 0.0263 & 0.00066 & 1.31\end{array}$ $\begin{array}{lllllllllll}2014 T 171-012 & 0.0725 & 0.00132 & 1.54098 & 0.0244 & 0.1542 & 0.00107 & 0.0474 & 0.00041 & 1.06\end{array}$ $\begin{array}{lllllllllll}2014 T 171-020 & 0.0767 & 0.00128 & 1.67833 & 0.0236 & 0.1588 & 0.00105 & 0.0478 & 0.00039 & 1.12\end{array}$ $\begin{array}{llllllllll}2014 T 171-087 & 0.0714 & 0.00738 & 1.582 & 0.1621 & 0.1607 & 0.00284 & 0.0384 & 0.00254 & 1.36\end{array}$ $\begin{array}{lllllllllll}2014 T 171-095 & 0.0719 & 0.00445 & 1.59199 & 0.0972 & 0.1607 & 0.00222 & 0.0239 & 0.00133 & 1.21\end{array}$ $\begin{array}{llllllllll}2014 T 171-016 & 0.0735 & 0.00157 & 1.73291 & 0.0334 & 0.171 & 0.00134 & 0.05 & 0.00053 & 1.02\end{array}$ $\begin{array}{lllllllllll}2014 T 171-033 & 0.0749 & 0.00123 & 1.85807 & 0.0254 & 0.1801 & 0.00118 & 0.0525 & 0.00121 & 9.29\end{array}$ $\begin{array}{llllllllll}2014 T 171-064 & 0.0757 & 0.00411 & 1.82999 & 0.0977 & 0.1753 & 0.002 & 0.0801 & 0.0029 & 2.58\end{array}$ $\begin{array}{lllllllllll}2014 T 171-058 & 0.0824 & 0.00272 & 2.43395 & 0.0775 & 0.2143 & 0.0019 & 0.0251 & 0.00462 & 4.73\end{array}$ $\begin{array}{lllllllllll}2014 T 171-032 & 0.0828 & 0.00122 & 2.22896 & 0.0261 & 0.1953 & 0.00123 & 0.0563 & 0.00076 & 4.21\end{array}$ $\begin{array}{llllllllll}2014 T 171-045 & 0.0845 & 0.00327 & 2.68068 & 0.1008 & 0.2301 & 0.0023 & -0.064 & 0.00533 & 6.72\end{array}$ $\begin{array}{llllllllll}2014 T 171-071 & 0.0885 & 0.00441 & 2.88761 & 0.1414 & 0.2367 & 0.00331 & 0.0444 & 0.00166 & 1.12\end{array}$ $\begin{array}{lllllllllll}2014 T 171-001 & 0.092 & 0.00152 & 3.03779 & 0.0427 & 0.2397 & 0.00174 & 0.0673 & 0.00071 & 1.44\end{array}$ $\begin{array}{llllllllll}2014 T 171-038 & 0.0945 & 0.00535 & 3.48381 & 0.1953 & 0.2677 & 0.00534 & 0.0901 & 0.00343 & 1.56\end{array}$ $\begin{array}{lllllllllll}2014 T 171-074 & 0.0945 & 0.00149 & 3.45426 & 0.0465 & 0.2651 & 0.00193 & 0.0787 & 0.00085 & 1.70\end{array}$ $\begin{array}{llllllllll}2014 T 171-026 & 0.0948 & 0.00186 & 3.1278 & 0.0563 & 0.2392 & 0.00184 & 0.0703 & 0.00049 & 2.79\end{array}$ $\begin{array}{llllllllll}2014 T 171-052 & 0.0999 & 0.00162 & 3.88668 & 0.0565 & 0.2822 & 0.00202 & 0.0824 & 0.00056 & 3.01\end{array}$ $\begin{array}{llllllllll}2014 T 171-030 & 0.1022 & 0.00367 & 3.7634 & 0.1313 & 0.2672 & 0.00396 & 0.0732 & 0.00191 & 1.23\end{array}$ $\begin{array}{lllllllllll}2014 T 171-008 & 0.1204 & 0.00177 & 5.50881 & 0.0706 & 0.3318 & 0.00237 & 0.0951 & 0.00065 & 4.16\end{array}$ $\begin{array}{llllllllll}2014 T 171-014 & 0.1409 & 0.00197 & 7.20435 & 0.0803 & 0.371 & 0.0027 & 0.1065 & 0.0013 & 2.04\end{array}$ $\begin{array}{llllllllll}2014 T 171-044 & 0.1524 & 0.00361 & 9.18287 & 0.2126 & 0.4374 & 0.00614 & 0.1357 & 0.00303 & 1.36\end{array}$ $\begin{array}{llllllllll}2014 T 171-009 & 0.1556 & 0.00204 & 8.95958 & 0.0905 & 0.4176 & 0.00295 & 0.114 & 0.00168 & 3.39\end{array}$ $\begin{array}{lllllllllll}2014 T 171-024 & 0.1877 & 0.00194 & 12.5464 & 0.1041 & 0.4848 & 0.00297 & 0.133 & 0.0008 & 2.63\end{array}$ $\begin{array}{lllllllllll}2014 T 171-011 & 0.1998 & 0.00294 & 14.2487 & 0.1801 & 0.5174 & 0.00466 & 0.135 & 0.0021 & 1.66\end{array}$

\begin{tabular}{cccccccccc}
762 & 50 & 756 & 16 & 755 & 6 & 129 & 11 & 755 & 12 \\
970 & 57 & 828 & 20 & 776 & 7 & 427 & 14 & 776 & 14 \\
846 & 41 & 811 & 10 & 799 & 5 & 797 & 5 & 799 & 10 \\
792 & 100 & 820 & 33 & 830 & 11 & 182 & 29 & 830 & 22 \\
950 & 18 & 878 & 8 & 850 & 5 & 751 & 18 & 850 & 10 \\
890 & 41 & 875 & 16 & 870 & 7 & 524 & 13 & 870 & 14 \\
1001 & 21 & 947 & 10 & 924 & 6 & 936 & 8 & 924 & 12 \\
1113 & 18 & 1000 & 9 & 950 & 6 & 944 & 8 & 950 & 12 \\
969 & 184 & 963 & 64 & 961 & 16 & 762 & 49 & 961 & 32 \\
982 & 102 & 967 & 38 & 961 & 12 & 478 & 26 & 961 & 24 \\
1028 & 26 & 1021 & 12 & 1018 & 7 & 987 & 10 & 1028 & 52 \\
1064 & 17 & 1066 & 9 & 1067 & 6 & 1035 & 23 & 1064 & 34 \\
1088 & 89 & 1056 & 35 & 1041 & 11 & 1558 & 54 & 1088 & 178 \\
1255 & 48 & 1253 & 23 & 1252 & 10 & 500 & 91 & 1255 & 96 \\
1265 & 13 & 1190 & 8 & 1150 & 7 & 1107 & 15 & 1265 & 26 \\
1305 & 58 & 1323 & 28 & 1335 & 12 & -1339 & 115 & 1305 & 116 \\
1393 & 72 & 1379 & 37 & 1370 & 17 & 878 & 32 & 1393 & 144 \\
1467 & 16 & 1417 & 11 & 1385 & 9 & 1317 & 13 & 1467 & 32 \\
1517 & 76 & 1524 & 44 & 1529 & 27 & 1743 & 64 & 1517 & 152 \\
1519 & 15 & 1517 & 11 & 1516 & 10 & 1531 & 16 & 1519 & 30 \\
1525 & 38 & 1440 & 14 & 1383 & 10 & 1372 & 9 & 1525 & 76 \\
1622 & 31 & 1611 & 12 & 1602 & 10 & 1601 & 10 & 1622 & 62 \\
1664 & 43 & 1585 & 28 & 1527 & 20 & 1429 & 36 & 1664 & 86 \\
1962 & 27 & 1902 & 11 & 1847 & 11 & 1836 & 12 & 1962 & 54 \\
2238 & 10 & 2137 & 10 & 2034 & 13 & 2045 & 24 & 2238 & 20 \\
2373 & 21 & 2356 & 21 & 2339 & 28 & 2573 & 54 & 2373 & 42 \\
2409 & 8 & 2334 & 9 & 2250 & 13 & 2182 & 30 & 2409 & 16 \\
2722 & 17 & 2646 & 8 & 2548 & 13 & 2524 & 14 & 2722 & 34 \\
2824 & 10 & 2766 & 12 & 2688 & 20 & 2559 & 37 & 2824 & 20 \\
\hline & & & & & & & & &
\end{tabular}

\section{$2014 T 172$ (Liuqu diorite clasts, Xialu section, $n=94, \mathrm{~N}^{\circ} 9^{\circ} 06.160^{\prime} \quad \mathrm{E88}{ }^{\circ} 58.104^{\prime}, 4148 \mathrm{~m}$ )}

$\begin{array}{llllllllll}2014 T 172-001 & 0.0486 & 0.00649 & 0.13694 & 0.0182 & 0.0204 & 0.00028 & 0.0068 & 0.00015 & 0.65\end{array}$ $\begin{array}{llllllllll}2014 T 172-002 & 0.0496 & 0.09889 & 0.15646 & 0.3119 & 0.0229 & 0.00238 & 0.0183 & 0.00902 & 2.73\end{array}$ $\begin{array}{llllllllll}2014 T 172-003 & 0.0489 & 0.04603 & 0.14279 & 0.1341 & 0.0212 & 0.00123 & 0.0099 & 0.00178 & 1.43\end{array}$

$\begin{array}{cccccccccc}129 & 255 & 130 & 16 & 130 & 2 & 137 & 3 & 130 & 4 \\ 174 & 2080 & 148 & 274 & 146 & 15 & 367 & 179 & 146 & 30 \\ 144 & 1162 & 136 & 119 & 135 & 8 & 199 & 36 & 135 & 16\end{array}$


$\begin{array}{llllllllll}2014 T 172-004 & 0.0491 & 0.0935 & 0.1805 & 0.3431 & 0.0267 & 0.00287 & 0.0524 & 0.0109 & 2.31\end{array}$ $\begin{array}{llllllllll}2014 T 172-005 & 0.0496 & 0.13004 & 0.1874 & 0.4905 & 0.0274 & 0.00346 & 0.0548 & 0.01328 & 2.31\end{array}$ $\begin{array}{lllllllllll}2014 \mathrm{~T} 172-006 & 0.0482 & 0.13379 & 0.14612 & 0.4047 & 0.022 & 0.00328 & 0.0298 & 0.00591 & 1.4\end{array}$ $\begin{array}{lllllllllll}2014 T 172-007 & 0.0502 & 0.07159 & 0.1636 & 0.2331 & 0.0237 & 0.00211 & 0.0252 & 0.00724 & 2.43\end{array}$ $\begin{array}{llllllllll}2014 \mathrm{~T} 172-008 & 0.052 & 0.06808 & 0.18751 & 0.2448 & 0.0261 & 0.00246 & 0.0295 & 0.00595 & 1.95\end{array}$ $\begin{array}{lllllllllll}2014 T 172-009 & 0.0519 & 0.06209 & 0.16901 & 0.2016 & 0.0236 & 0.00206 & 0.0169 & 0.00604 & 2.47\end{array}$ $\begin{array}{llllllllll}2014 T 172-010 & 0.051 & 0.08729 & 0.17381 & 0.2972 & 0.0247 & 0.00243 & 0.0435 & 0.00746 & 2.36\end{array}$ $\begin{array}{llllllllll}2014 \mathrm{~T} 172-012 & 0.0452 & 0.05249 & 0.16807 & 0.195 & 0.027 & 0.00226 & 0.0261 & 0.00587 & 2.1\end{array}$ $\begin{array}{llllllllll}2014 T 172-013 & 0.052 & 0.08583 & 0.19499 & 0.3216 & 0.0272 & 0.00258 & 0.0232 & 0.00714 & 2\end{array}$ $\begin{array}{llllllllll}2014 T 172-014 & 0.0517 & 0.04772 & 0.18111 & 0.1667 & 0.0254 & 0.00199 & 0.019 & 0.00405 & 1.89\end{array}$

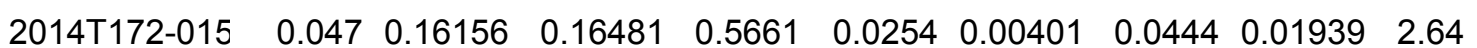
$\begin{array}{llllllllll}2014 \mathrm{~T} 172-018 & 0.0451 & 0.11117 & 0.14898 & 0.3666 & 0.0239 & 0.00284 & 0.0233 & 0.00801 & 1.75\end{array}$ $\begin{array}{llllllllll}2014 \mathrm{~T} 172-019 & 0.0456 & 0.16967 & 0.13825 & 0.5138 & 0.022 & 0.00423 & 0.0131 & 0.01413 & 1.92\end{array}$ $\begin{array}{lllllllllll}2014 \mathrm{~T} 172-020 & 0.054 & 0.15097 & 0.18955 & 0.5293 & 0.0255 & 0.00392 & 0.0486 & 0.00997 & 1.84\end{array}$ $\begin{array}{llllllllll}2014 T 172-021 & 0.0505 & 0.08392 & 0.1931 & 0.3201 & 0.0277 & 0.00285 & 0.0259 & 0.00853 & 2\end{array}$ $\begin{array}{llllllllll}2014 T 172-022 & 0.045 & 0.08587 & 0.14037 & 0.2676 & 0.0226 & 0.00218 & 0.0177 & 0.00465 & 1.82\end{array}$ $\begin{array}{llllllllll}2014 T 172-023 & 0.0538 & 0.01327 & 0.16078 & 0.0395 & 0.0217 & 0.00054 & 0.0071 & 0.00042 & 1\end{array}$ $\begin{array}{lllllllllll}2014 T 172-024 & 0.0475 & 0.00701 & 0.13953 & 0.0205 & 0.0213 & 0.00029 & 0.0067 & 0.00013 & 0.57\end{array}$ $\begin{array}{lllllllllll}2014 T 172-025 & 0.0467 & 0.07204 & 0.16237 & 0.2501 & 0.0252 & 0.00206 & 0.0153 & 0.00275 & 1.13\end{array}$ $\begin{array}{llllllllll}2014 \mathrm{~T} 172-026 & 0.0527 & 0.09326 & 0.20849 & 0.3686 & 0.0287 & 0.00325 & 0.0091 & 0.00761 & 1.63\end{array}$ $\begin{array}{llllllllll}2014 \mathrm{~T} 172-028 & 0.052 & 0.03631 & 0.15976 & 0.1113 & 0.0223 & 0.00092 & 0.0079 & 0.00152 & 1.3\end{array}$ $\begin{array}{lllllllllll}2014 T 172-029 & 0.0464 & 0.09028 & 0.13591 & 0.2639 & 0.0212 & 0.00223 & 0.0253 & 0.00703 & 2.2\end{array}$ $\begin{array}{llllllllll}2014 \mathrm{~T} 172-030 & 0.0484 & 0.19614 & 0.12445 & 0.504 & 0.0187 & 0.0039 & 0.0214 & 0.01165 & 1.97\end{array}$ $\begin{array}{lllllllllll}2014 T 172-031 & 0.0537 & 0.09169 & 0.16233 & 0.2766 & 0.0219 & 0.0022 & 0.0303 & 0.00753 & 2.5\end{array}$ $\begin{array}{lllllllllll}2014 \mathrm{~T} 172-032 & 0.0482 & 0.08851 & 0.14715 & 0.27 & 0.0222 & 0.00225 & 0.0275 & 0.00556 & 2\end{array}$ $\begin{array}{lllllllllll}2014 T 172-033 & 0.0489 & 0.07046 & 0.18736 & 0.2698 & 0.0278 & 0.00191 & 0.0234 & 0.00446 & 1.75\end{array}$ $\begin{array}{lllllllllll}2014 T 172-034 & 0.0425 & 0.16331 & 0.10038 & 0.3855 & 0.0171 & 0.00316 & 0.0318 & 0.0068 & 1.7\end{array}$ $\begin{array}{lllllllllll}2014 T 172-035 & 0.0554 & 0.16257 & 0.21938 & 0.6431 & 0.0287 & 0.00469 & 0.0532 & 0.01159 & 1.65\end{array}$ $\begin{array}{lllllllllll}2014 T 172-036 & 0.0461 & 0.13003 & 0.16098 & 0.4535 & 0.0253 & 0.00355 & 0.0333 & 0.00955 & 1.94\end{array}$ $\begin{array}{llllllllll}2014 T 172-037 & 0.0522 & 0.033 & 0.15454 & 0.0977 & 0.0215 & 0.0007 & 0.0075 & 0.00064 & 0.76\end{array}$ $\begin{array}{llllllllll}2014 T 172-038 & 0.054 & 0.13506 & 0.17681 & 0.4414 & 0.0237 & 0.0036 & 0.029 & 0.00973 & 2.15\end{array}$ $\begin{array}{lllllllllll}2014 T 172-039 & 0.0467 & 0.00358 & 0.13608 & 0.0103 & 0.0211 & 0.00027 & 0.0069 & 0.00011 & 0.52\end{array}$ $\begin{array}{llllllllll}2014 \mathrm{~T} 172-041 & 0.0471 & 0.21782 & 0.13871 & 0.6407 & 0.0214 & 0.00519 & 0.0462 & 0.01718 & 2.21\end{array}$

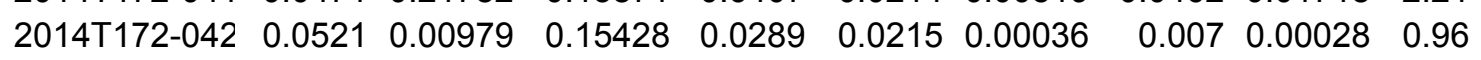

$\begin{array}{cccccccccc}153 & 1956 & 168 & 295 & 170 & 18 & 1033 & 209 & 170 & 36 \\ 177 & 3075 & 174 & 419 & 174 & 22 & 1078 & 254 & 174 & 44 \\ 111 & 3243 & 138 & 359 & 140 & 21 & 593 & 116 & 140 & 42 \\ 202 & 1503 & 154 & 203 & 151 & 13 & 503 & 143 & 151 & 26 \\ 286 & 1477 & 175 & 209 & 166 & 15 & 587 & 117 & 166 & 30 \\ 282 & 1376 & 159 & 175 & 150 & 13 & 339 & 120 & 150 & 26 \\ 239 & 1804 & 163 & 257 & 157 & 15 & 860 & 145 & 157 & 30 \\ -12 & 1269 & 158 & 169 & 172 & 14 & 521 & 116 & 172 & 28 \\ 284 & 1771 & 181 & 273 & 173 & 16 & 463 & 141 & 173 & 32 \\ 271 & 1174 & 169 & 143 & 162 & 13 & 379 & 80 & 162 & 26 \\ 48 & 3431 & 155 & 493 & 162 & 25 & 878 & 375 & 162 & 50 \\ -13 & 2539 & 141 & 324 & 152 & 18 & 465 & 158 & 152 & 36 \\ -23 & 3487 & 131 & 458 & 140 & 27 & 264 & 282 & 140 & 54 \\ 371 & 3185 & 176 & 452 & 162 & 25 & 959 & 192 & 162 & 50 \\ 220 & 1730 & 179 & 272 & 176 & 18 & 518 & 168 & 176 & 36 \\ -20 & 1817 & 133 & 238 & 144 & 14 & 355 & 92 & 144 & 28 \\ 362 & 424 & 151 & 35 & 138 & 3 & 142 & 8 & 138 & 6 \\ 75 & 269 & 133 & 18 & 136 & 2 & 134 & 3 & 136 & 4 \\ 34 & 1514 & 153 & 218 & 160 & 13 & 308 & 55 & 160 & 26 \\ 314 & 1925 & 192 & 310 & 182 & 20 & 184 & 152 & 182 & 40 \\ 287 & 1050 & 150 & 97 & 142 & 6 & 159 & 30 & 142 & 12 \\ 20 & 1903 & 129 & 236 & 135 & 14 & 504 & 139 & 135 & 28 \\ 117 & 3725 & 119 & 455 & 119 & 25 & 428 & 231 & 119 & 50 \\ 359 & 1885 & 153 & 242 & 140 & 14 & 603 & 148 & 140 & 28 \\ 108 & 1859 & 139 & 239 & 141 & 14 & 548 & 109 & 141 & 28 \\ 142 & 1508 & 174 & 231 & 177 & 12 & 468 & 88 & 177 & 24 \\ -151 & 3453 & 97 & 356 & 110 & 20 & 633 & 133 & 110 & 40 \\ 427 & 3370 & 201 & 536 & 183 & 29 & 1047 & 222 & 183 & 58 \\ 3 & 3129 & 152 & 397 & 161 & 22 & 662 & 187 & 161 & 44 \\ 292 & 1018 & 146 & 86 & 137 & 4 & 151 & 13 & 137 & 8 \\ 371 & 3150 & 165 & 381 & 151 & 23 & 577 & 191 & 151 & 46 \\ 33 & 141 & 130 & 9 & 135 & 2 & 138 & 2 & 135 & 4 \\ 54 & 3659 & 132 & 571 & 136 & 33 & 912 & 332 & 136 & 66 \\ 289 & 351 & 146 & 25 & 137 & 2 & 141 & 6 & 137 & 4\end{array}$


$\begin{array}{llllllllll}2014 T 172-043 & 0.0487 & 0.01981 & 0.12821 & 0.052 & 0.0191 & 0.0005 & 0.0048 & 0.00023 & 0.43\end{array}$ $\begin{array}{llllllllll}2014 \mathrm{~T} 172-044 & 0.0461 & 0.02393 & 0.09499 & 0.048 & 0.015 & 0.0018 & 0.0301 & 0.01467 & 1.82\end{array}$ $\begin{array}{lllllllllll}2014 \mathrm{~T} 172-045 & 0.0537 & 0.00555 & 0.16785 & 0.0171 & 0.0227 & 0.00039 & 0.0078 & 0.0002 & 0.69\end{array}$ $\begin{array}{llllllllllll}2014 T 172-046 & 0.0514 & 0.00326 & 0.14476 & 0.009 & 0.0204 & 0.00024 & 0.0061 & 0.00008 & 0.33\end{array}$ $\begin{array}{lllllllllll}2014 \mathrm{~T} 172-047 & 0.0435 & 0.02008 & 0.12523 & 0.0578 & 0.0209 & 0.00054 & 0.0068 & 0.00037 & 0.73\end{array}$ $\begin{array}{llllllllll}2014 T 172-048 & 0.0526 & 0.00417 & 0.15461 & 0.0121 & 0.0213 & 0.00029 & 0.0067 & 0.00013 & 0.62\end{array}$ $\begin{array}{llllllllll}2014 T 172-049 & 0.0536 & 0.09975 & 0.18606 & 0.3458 & 0.0252 & 0.00306 & 0.0687 & 0.0091 & 2.39\end{array}$ $\begin{array}{lllllllllll}2014 T 172-050 & 0.0477 & 0.14752 & 0.13525 & 0.4181 & 0.0206 & 0.0031 & 0.0254 & 0.00895 & 1.93\end{array}$ $\begin{array}{llllllllll}2014 \mathrm{~T} 172-052 & 0.0506 & 0.06817 & 0.18416 & 0.2478 & 0.0264 & 0.00224 & 0.0212 & 0.005 & 1.78\end{array}$ $\begin{array}{lllllllllll}2014 T 172-053 & 0.0527 & 0.0523 & 0.20066 & 0.1989 & 0.0276 & 0.00167 & 0.0137 & 0.00104 & 0.63\end{array}$ $\begin{array}{llllllllll}2014 T 172-054 & 0.0538 & 0.08301 & 0.15443 & 0.2377 & 0.0208 & 0.00198 & 0.0193 & 0.00353 & 1.55\end{array}$ $\begin{array}{lllllllllll}2014 T 172-055 & 0.0499 & 0.07458 & 0.16191 & 0.2417 & 0.0235 & 0.0018 & 0.0131 & 0.00161 & 0.98\end{array}$ $\begin{array}{lllllllllll}2014 T 172-056 & 0.0502 & 0.15218 & 0.14082 & 0.4265 & 0.0204 & 0.00344 & 0.0234 & 0.01166 & 2.29\end{array}$ $\begin{array}{lllllllllll}2014 \mathrm{~T} 172-057 & 0.0543 & 0.0038 & 0.15099 & 0.0103 & 0.0202 & 0.00026 & 0.0061 & 0.00009 & 0.38\end{array}$ $\begin{array}{lllllllllll}2014 \mathrm{~T} 172-058 & 0.0534 & 0.01052 & 0.15898 & 0.0312 & 0.0216 & 0.00035 & 0.0067 & 0.00016 & 0.53\end{array}$ $\begin{array}{llllllllll}2014 T 172-059 & 0.0982 & 0.18157 & 0.28497 & 0.5239 & 0.021 & 0.00423 & 0.0284 & 0.01166 & 1.89\end{array}$ $\begin{array}{llllllllll}2014 \mathrm{~T} 172-060 & 0.0462 & 0.06162 & 0.14758 & 0.1966 & 0.0232 & 0.00192 & 0.0198 & 0.00487 & 2.08\end{array}$ $\begin{array}{lllllllllll}2014 \mathrm{~T} 172-061 & 0.051 & 0.00384 & 0.14917 & 0.011 & 0.0212 & 0.00027 & 0.0068 & 0.00018 & 1.11\end{array}$ $\begin{array}{llllllllll}2014 T 172-063 & 0.051 & 0.16922 & 0.15725 & 0.5207 & 0.0223 & 0.004 & 0.048 & 0.01163 & 1.92\end{array}$ $\begin{array}{llllllllll}2014 T 172-064 & 0.0481 & 0.13916 & 0.17315 & 0.5007 & 0.0261 & 0.00357 & 0.027 & 0.01323 & 2.21\end{array}$ $\begin{array}{lllllllllll}2014 T 172-065 & 0.0473 & 0.14322 & 0.13806 & 0.417 & 0.0212 & 0.00387 & -0.001 & 0.01476 & 2.26\end{array}$ $\begin{array}{llllllllll}2014 \mathrm{~T} 172-066 & 0.0439 & 0.09822 & 0.15842 & 0.354 & 0.0262 & 0.00259 & 0.0219 & 0.00659 & 1.84\end{array}$ $\begin{array}{lllllllllll}2014 T 172-067 & 0.052 & 0.11829 & 0.16717 & 0.3796 & 0.0233 & 0.00265 & 0.0058 & 0.01038 & 2.05\end{array}$ $\begin{array}{llllllllll}2014 T 172-068 & 0.0479 & 0.00257 & 0.13032 & 0.0068 & 0.0197 & 0.0002 & 0.0057 & 0.00009 & 0.63\end{array}$ $\begin{array}{lllllllllll}2014 T 172-069 & 0.0569 & 0.00475 & 0.15688 & 0.0129 & 0.02 & 0.00029 & 0.0059 & 0.00015 & 0.76\end{array}$ $\begin{array}{llllllllll}2014 T 172-070 & 0.0534 & 0.15067 & 0.24376 & 0.6867 & 0.0331 & 0.0047 & 0.0496 & 0.01185 & 1.68\end{array}$ $\begin{array}{lllllllllll}2014 T 172-071 & 0.0522 & 0.0078 & 0.15169 & 0.0225 & 0.0211 & 0.00034 & 0.0061 & 0.00017 & 0.59\end{array}$ $\begin{array}{lllllllllll}2014 T 172-072 & 0.0462 & 0.07166 & 0.15526 & 0.2406 & 0.0244 & 0.00174 & 0.0108 & 0.00571 & 2.17\end{array}$ $\begin{array}{llllllllll}2014 T 172-073 & 0.0463 & 0.03209 & 0.15408 & 0.1066 & 0.0241 & 0.00106 & 0.01 & 0.00198 & 1.83\end{array}$ $\begin{array}{llllllllll}2014 T 172-074 & 0.0514 & 0.1571 & 0.1881 & 0.5744 & 0.0265 & 0.0037 & -0.008 & 0.01667 & 2\end{array}$ $\begin{array}{llllllllll}2014 \mathrm{~T} 172-075 & 0.0454 & 0.07162 & 0.1104 & 0.1739 & 0.0176 & 0.00159 & 0.0077 & 0.00474 & 2.41\end{array}$ $\begin{array}{llllllllll}2014 T 172-076 & 0.0477 & 0.07341 & 0.12324 & 0.1894 & 0.0187 & 0.00184 & 0.0109 & 0.00442 & 2.16\end{array}$ $\begin{array}{llllllllll}2014 T 172-077 & 0.0508 & 0.08895 & 0.18254 & 0.3194 & 0.0261 & 0.00261 & 0.0208 & 0.00508 & 1.53\end{array}$

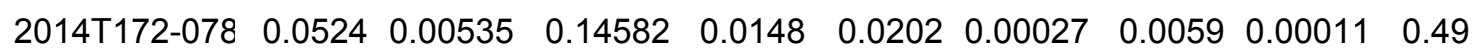

$\begin{array}{cccccccccc}134 & 646 & 122 & 47 & 122 & 3 & 98 & 5 & 122 & 6 \\ & 874 & 92 & 45 & 96 & 11 & 599 & 288 & 96 & 22 \\ 360 & 199 & 158 & 15 & 144 & 2 & 157 & 4 & 144 & 4 \\ 257 & 121 & 137 & 8 & 130 & 2 & 123 & 2 & 130 & 4 \\ -99 & 703 & 120 & 52 & 133 & 3 & 137 & 7 & 133 & 6 \\ 311 & 153 & 146 & 11 & 136 & 2 & 135 & 3 & 136 & 4 \\ 353 & 2062 & 173 & 296 & 160 & 19 & 1343 & 172 & 160 & 38 \\ 83 & 3214 & 129 & 374 & 131 & 20 & 507 & 176 & 131 & 40 \\ 222 & 1462 & 172 & 212 & 168 & 14 & 424 & 99 & 168 & 28 \\ 315 & 1274 & 186 & 168 & 176 & 10 & 274 & 21 & 176 & 20 \\ 364 & 1653 & 146 & 209 & 133 & 13 & 385 & 70 & 133 & 26 \\ 190 & 1566 & 152 & 211 & 150 & 11 & 262 & 32 & 150 & 22 \\ 203 & 3315 & 134 & 380 & 130 & 22 & 468 & 230 & 130 & 44 \\ 384 & 132 & 143 & 9 & 129 & 2 & 123 & 2 & 129 & 4 \\ 347 & 371 & 150 & 27 & 138 & 2 & 134 & 3 & 138 & 4 \\ 1590 & 3399 & 255 & 414 & 134 & 27 & 567 & 229 & 134 & 54 \\ 7 & 1420 & 140 & 174 & 148 & 12 & 396 & 97 & 148 & 24 \\ 242 & 145 & 141 & 10 & 135 & 2 & 136 & 4 & 135 & 4 \\ 242 & 3460 & 148 & 457 & 142 & 25 & 948 & 224 & 142 & 50 \\ 103 & 3394 & 162 & 433 & 166 & 22 & 539 & 260 & 166 & 44 \\ 66 & 3379 & 131 & 372 & 135 & 24 & -27 & 299 & 135 & 48 \\ -75 & 2140 & 149 & 310 & 166 & 16 & 438 & 130 & 166 & 32 \\ 287 & 2704 & 157 & 330 & 148 & 17 & 116 & 209 & 148 & 34 \\ 96 & 97 & 124 & 6 & 126 & 1 & 115 & 2 & 126 & 2 \\ 488 & 157 & 148 & 11 & 128 & 2 & 119 & 3 & 128 & 4 \\ 347 & 3214 & 221 & 561 & 210 & 29 & 979 & 228 & 210 & 58 \\ 295 & 300 & 143 & 20 & 134 & 2 & 122 & 3 & 134 & 4 \\ 9 & 1513 & 147 & 211 & 155 & 11 & 218 & 114 & 155 & 22 \\ 13 & 1011 & 146 & 94 & 154 & 7 & 202 & 40 & 154 & 14 \\ 258 & 3439 & 175 & 491 & 169 & 23 & -159 & 340 & 169 & 46 \\ -34 & 1507 & 106 & 159 & 113 & 10 & 156 & 95 & 113 & 20 \\ 83 & 1536 & 118 & 171 & 120 & 12 & 220 & 88 & 120 & 24 \\ 230 & 1857 & 170 & 274 & 166 & 16 & 416 & 101 & 166 & 32 \\ 301 & 204 & 138 & 13 & 129 & 2 & 119 & 2 & 129 & 4\end{array}$


$\begin{array}{llllllllll}2014 T 172-079 & 0.0452 & 0.01365 & 0.13806 & 0.0416 & 0.0221 & 0.00048 & 0.0067 & 0.00038 & 0.95\end{array}$ $\begin{array}{lllllllllll}2014 \mathrm{~T} 172-080 & 0.054 & 0.01464 & 0.1544 & 0.0417 & 0.0207 & 0.00042 & 0.006 & 0.00026 & 0.63\end{array}$ $\begin{array}{llllllllll}2014 \mathrm{~T} 172-081 & 0.0456 & 0.02141 & 0.11157 & 0.0522 & 0.0177 & 0.00059 & 0.0068 & 0.00062 & 1.29\end{array}$ $\begin{array}{lllllllllll}2014 T 172-082 & 0.0489 & 0.08093 & 0.15574 & 0.2573 & 0.0231 & 0.00199 & 0.0424 & 0.00486 & 2.27\end{array}$ $\begin{array}{lllllllllll}2014 \mathrm{~T} 172-083 & 0.0461 & 0.00254 & 0.12626 & 0.0069 & 0.0199 & 0.00017 & 0.0064 & 0.00007 & 0.48\end{array}$ $\begin{array}{llllllllll}2014 T 172-084 & 0.0469 & 0.08118 & 0.16775 & 0.2904 & 0.026 & 0.00194 & 0.0198 & 0.00665 & 2.22\end{array}$ $\begin{array}{llllllllll}2014 T 172-085 & 0.0482 & 0.16382 & 0.17135 & 0.5815 & 0.0258 & 0.0042 & 0.0558 & 0.00945 & 1.85\end{array}$ $\begin{array}{llllllllll}2014 \mathrm{~T} 172-086 & 0.0505 & 0.06439 & 0.16253 & 0.2069 & 0.0233 & 0.0015 & 0.0145 & 0.00455 & 2.22\end{array}$ $\begin{array}{llllllllll}2014 T 172-087 & 0.0459 & 0.20594 & 0.11939 & 0.5348 & 0.0189 & 0.00382 & 0.0472 & 0.01196 & 2.38\end{array}$ $\begin{array}{lllllllllll}2014 T 172-088 & 0.0455 & 0.09558 & 0.13182 & 0.2768 & 0.021 & 0.00213 & 0.0057 & 0.00745 & 2.39\end{array}$ $\begin{array}{lllllllllll}2014 T 172-089 & 0.0512 & 0.00719 & 0.14167 & 0.0198 & 0.0201 & 0.00029 & 0.0063 & 0.00013 & 0.47\end{array}$ $\begin{array}{lllllllllll}2014 \mathrm{~T} 172-090 & 0.0497 & 0.00697 & 0.14127 & 0.0197 & 0.0206 & 0.00031 & 0.0055 & 0.00012 & 0.52\end{array}$ $\begin{array}{lllllllllll}2014 T 172-091 & 0.0521 & 0.12356 & 0.17873 & 0.4233 & 0.0249 & 0.00307 & 0.0219 & 0.00776 & 1.83\end{array}$ $\begin{array}{llllllllll}2014 \mathrm{~T} 172-092 & 0.0517 & 0.00794 & 0.17603 & 0.0267 & 0.0247 & 0.00059 & 0.0072 & 0.00025 & 0.51\end{array}$ $\begin{array}{llllllllllll}2014 T 172-093 & 0.0519 & 0.06389 & 0.1399 & 0.172 & 0.0196 & 0.00159 & 0.0217 & 0.00494 & 2.43\end{array}$ $\begin{array}{llllllllll}2014 T 172-094 & 0.0466 & 0.00421 & 0.1322 & 0.0118 & 0.0206 & 0.00028 & 0.0058 & 0.00012 & 0.54\end{array}$ $\begin{array}{llllllllllll}2014 T 172-095 & 0.0526 & 0.00565 & 0.1491 & 0.0159 & 0.0206 & 0.00028 & 0.006 & 0.00013 & 0.57\end{array}$ $\begin{array}{lllllllllll}2014 \mathrm{~T} 172-096 & 0.048 & 0.12248 & 0.14883 & 0.3796 & 0.0225 & 0.00278 & 0.0283 & 0.0089 & 2.21\end{array}$ $\begin{array}{lllllllllll}2014 T 172-097 & 0.0495 & 0.0182 & 0.16844 & 0.0616 & 0.0247 & 0.00088 & 0.0093 & 0.00089 & 1.27\end{array}$ $\begin{array}{lllllllllll}2014 T 172-099 & 0.0447 & 0.13182 & 0.15894 & 0.4687 & 0.0258 & 0.00338 & 0.0272 & 0.00877 & 1.77\end{array}$ $\begin{array}{lllllllllll}2014 \mathrm{~T} 172-100 & 0.0526 & 0.0057 & 0.15633 & 0.0167 & 0.0215 & 0.00038 & 0.0065 & 0.00019 & 0.65\end{array}$

\begin{tabular}{cccccccccc}
-8 & 441 & 131 & 37 & 141 & 3 & 135 & 8 & 141 & 6 \\
372 & 474 & 146 & 37 & 132 & 3 & 122 & 5 & 132 & 6 \\
-22 & 727 & 107 & 48 & 113 & 4 & 138 & 12 & 113 & 8 \\
144 & 1695 & 147 & 226 & 147 & 13 & 839 & 94 & 147 & 26 \\
& 120 & 121 & 6 & 127 & 1 & 128 & 1 & 127 & 2 \\
42 & 1735 & 157 & 252 & 165 & 12 & 396 & 132 & 165 & 24 \\
110 & 3342 & 161 & 504 & 164 & 26 & 1097 & 181 & 164 & 52 \\
219 & 1438 & 153 & 181 & 149 & 9 & 291 & 91 & 149 & 18 \\
-5 & 3623 & 115 & 485 & 120 & 24 & 932 & 231 & 120 & 48 \\
-30 & 2038 & 126 & 248 & 134 & 13 & 115 & 150 & 134 & 26 \\
250 & 283 & 135 & 18 & 128 & 2 & 127 & 3 & 128 & 4 \\
180 & 268 & 134 & 18 & 132 & 2 & 110 & 2 & 132 & 4 \\
290 & 2746 & 167 & 365 & 158 & 19 & 437 & 153 & 158 & 38 \\
272 & 292 & 165 & 23 & 157 & 4 & 145 & 5 & 157 & 8 \\
279 & 1406 & 133 & 153 & 125 & 10 & 434 & 98 & 125 & 20 \\
29 & 172 & 126 & 11 & 131 & 2 & 116 & 2 & 131 & 4 \\
312 & 216 & 141 & 14 & 131 & 2 & 122 & 3 & 131 & 4 \\
97 & 2782 & 141 & 336 & 143 & 18 & 563 & 175 & 143 & 36 \\
173 & 571 & 158 & 54 & 157 & 6 & 187 & 18 & 157 & 12 \\
-37 & 3276 & 150 & 411 & 164 & 21 & 543 & 173 & 164 & 42 \\
313 & 209 & 147 & 15 & 137 & 2 & 131 & 4 & 137 & 4 \\
\hline
\end{tabular}

2009TL1126 (Liuqu, upper part of the Za section, $\mathrm{n}=50, \mathrm{~N}^{\circ} 9^{\circ} 09.935^{\prime} \quad \mathrm{E88}^{\circ} 08.450^{\prime}$, $4229 \mathrm{~m}$ )

$\begin{array}{llllllllll}10 \mathrm{~L} 06008 & 0.0625 & 0.0010 & 0.7458 & 0.0131 & 0.0836 & 0.0010 & 0.0253 & 0.0003 & 1.05\end{array}$

$10 \mathrm{~L} 06013$

$\begin{array}{lll}0.0844 & 0.0040\end{array}$

$\begin{array}{llllll}1.9428 & 0.0863 & 0.1669 & 0.0024 & 0.0496\end{array}$

$0.0006 \quad 1.00$

$10 \mathrm{~L} 06017$

$10 \mathrm{~L} 06018$

$10 \mathrm{~L} 06022$

$10 \mathrm{~L} 06025$

$\begin{array}{ll}0.0734 & 0.0009\end{array}$

$\begin{array}{lllllll}1.5085 & 0.0205 & 0.1439 & 0.0017 & 0.0401 & 0.0004 & 1.17\end{array}$

$0.0650 \quad 0.0009$

$\begin{array}{llll}0.9867 & 0.0156 & 0.1062\end{array}$

$0.0766 \quad 0.0010$

$\begin{array}{lll}1.7245 & 0.0240 & 0.1576\end{array}$

$\begin{array}{ll}0.0776 & 0.0009\end{array}$

$\begin{array}{lll}1.7883 & 0.0233 & 0.1613\end{array}$

$0.0013 \quad 0.0315$

$0.0003 \quad 1.72$

$0.0624 \quad 0.0009$

$\begin{array}{lll}0.7508 & 0.0117 & 0.0842\end{array}$

$\begin{array}{lll}0.0019 & 0.0344\end{array}$

$\begin{array}{lll}0.0019 & 0.0422\end{array}$

$0.0004 \quad 4.40$

$10 \mathrm{~L} 06032$

$0.0729 \quad 0.0010$

$\begin{array}{lll}1.4165 & 0.0218 & 0.1359\end{array}$

$\begin{array}{llll}0.0010 & 0.0232 & 0.0003 & 3.71\end{array}$

$10 \mathrm{~L} 06034$

$0.0642 \quad 0.0009$

$\begin{array}{llllllll}1.0847 & 0.0170 & 0.1183 & 0.0014 & 0.0298 & 0.0004 & 2.57\end{array}$

$\begin{array}{ll}0.0617 & 0.0009\end{array}$

$\begin{array}{lll}1.0112 & 0.0164 & 0.1148\end{array}$

\begin{tabular}{llll}
0.0014 & 0.0302 & 0.0004 & 2.91 \\
\hline
\end{tabular}

10L06041

$\begin{array}{lll}2.0664 & 0.0340 & 0.1824\end{array}$

$\begin{array}{lllll}0.0022 & 0.0490 & 0.0005 & 0.69\end{array}$

$\begin{array}{cccccccccc}692 & 19 & 566 & 8 & 517 & 6 & 506 & 5 & 517 & 12 \\ 1302 & 93 & 1096 & 30 & 995 & 13 & 979 & 11 & 995 & 26 \\ 1025 & 12 & 934 & 8 & 867 & 9 & 794 & 7 & 867 & 18 \\ 775 & 16 & 697 & 8 & 651 & 7 & 627 & 6 & 651 & 14 \\ 1111 & 12 & 1018 & 9 & 943 & 10 & 684 & 9 & 943 & 20 \\ 1137 & 12 & 1041 & 8 & 964 & 10 & 836 & 8 & 964 & 20 \\ 689 & 15 & 569 & 7 & 521 & 6 & 464 & 6 & 521 & 12 \\ 1012 & 14 & 896 & 9 & 822 & 9 & 780 & 9 & 822 & 18 \\ 748 & 15 & 746 & 8 & 721 & 8 & 593 & 7 & 721 & 16 \\ 662 & 16 & 709 & 8 & 700 & 8 & 601 & 7 & 700 & 16 \\ 1179 & 15 & 1138 & 11 & 1080 & 12 & 967 & 9 & 1179 & 30\end{array}$


10L06042 10L06043

10L06048

10L06049

10L06053

10L06054

10L06055

10L06057

$10 \mathrm{~L} 06058$

10L06061

10L06064

$10 \mathrm{~L} 06066$

10L06068

$10 L 06069$

$10 \mathrm{~L} 06070$

10L06071

10L06072

10L06073

$10 \mathrm{~L} 06076$

$10 \mathrm{~L} 06078$

10L06079

$10 \mathrm{~L} 06080$

10L06081

10L06083

10L06088

$10 \mathrm{~L} 06090$

10L06092

10L06094

10L06095

10L06097

10L06100

10L06101

10L06102

10L06104 $\begin{array}{ll}0.0619 & 0.0008\end{array}$

$0.0953 \quad 0.0011$

$0.1012 \quad 0.0013$

$\begin{array}{ll}0.1484 & 0.0017\end{array}$

$0.0596 \quad 0.0016$

$0.0685 \quad 0.0010$

$0.0951 \quad 0.0009$

$0.0622 \quad 0.0010$

$0.0605 \quad 0.0008$

$0.0631 \quad 0.0010$

$\begin{array}{ll}0.0707 & 0.0009\end{array}$

$0.0796 \quad 0.0010$

$0.0758 \quad 0.0010$

$0.0722 \quad 0.0010$

$0.0907 \quad 0.0009$

$0.0741 \quad 0.0014$

$0.0725 \quad 0.0009$

$0.0764 \quad 0.0012$

$0.0676 \quad 0.0008$

$0.0674 \quad 0.0010$

$0.1659 \quad 0.0015$

0.06910 .0008

$0.1449 \quad 0.0014$

$\begin{array}{ll}0.0478 & 0.0014\end{array}$

$0.0628 \quad 0.0009$

$\begin{array}{ll}0.0610 & 0.0007\end{array}$

$0.0557 \quad 0.0009$

$0.0527 \quad 0.0006$

$0.0629 \quad 0.0014$

$0.0534 \quad 0.0010$

$\begin{array}{ll}0.1552 & 0.0014 \\ 0.1697 & 0.0017\end{array}$

$0.0504 \quad 0.0007$

$0.0589 \quad 0.0020$ $\begin{array}{lllllll}0.8356 & 0.0114 & 0.0945 & 0.0011 & 0.0251 & 0.0002 & 1.01\end{array}$ $\begin{array}{llllllll}3.3640 & 0.0445 & 0.2470 & 0.0030 & 0.0600 & 0.0006 & 1.48\end{array}$ $\begin{array}{llllllll}3.8238 & 0.0537 & 0.2645 & 0.0032 & 0.0768 & 0.0007 & 1.05\end{array}$ $\begin{array}{llllllll}8.3939 & 0.1077 & 0.3958 & 0.0048 & 0.0914 & 0.0008 & 0.78\end{array}$ $\begin{array}{llllllll}0.6520 & 0.0172 & 0.0765 & 0.0010 & 0.0200 & 0.0002 & 0.50\end{array}$ $\begin{array}{lllllll}1.5056 & 0.0238 & 0.1539 & 0.0019 & 0.0401 & 0.0004 & 1.26\end{array}$ $\begin{array}{llllllll}3.5828 & 0.0423 & 0.2638 & 0.0031 & 0.0705 & 0.0006 & 1.34\end{array}$ $\begin{array}{llllllll}0.7656 & 0.0126 & 0.0862 & 0.0011 & 0.0305 & 0.0003 & 2.02\end{array}$ $\begin{array}{llllllll}0.7813 & 0.0111 & 0.0904 & 0.0011 & 0.0197 & 0.0002 & 3.54\end{array}$ $\begin{array}{llllllll}0.7513 & 0.0130 & 0.0834 & 0.0010 & 0.0221 & 0.0002 & 1.44\end{array}$ $\begin{array}{llllllll}1.6774 & 0.0241 & 0.1662 & 0.0020 & 0.0418 & 0.0004 & 0.82\end{array}$ $\begin{array}{llllllll}2.1470 & 0.0298 & 0.1890 & 0.0023 & 0.0468 & 0.0004 & 1.44\end{array}$ $\begin{array}{llllllll}2.1756 & 0.0310 & 0.2012 & 0.0025 & 0.0447 & 0.0004 & 0.99\end{array}$ $\begin{array}{lllllll}1.6857 & 0.0251 & 0.1636 & 0.0020 & 0.0384 & 0.0004 & 1.08\end{array}$ $\begin{array}{lllllll}3.2814 & 0.0399 & 0.2536 & 0.0031 & 0.0517 & 0.0005 & 3.09\end{array}$ $\begin{array}{lllllll}1.9133 & 0.0364 & 0.1808 & 0.0023 & 0.0482 & 0.0005 & 0.73\end{array}$ $\begin{array}{lllllll}1.8422 & 0.0251 & 0.1781 & 0.0022 & 0.0415 & 0.0004 & 1.70\end{array}$ $\begin{array}{lllllll}1.8858 & 0.0313 & 0.1730 & 0.0022 & 0.0297 & 0.0004 & 1.12\end{array}$ $\begin{array}{lllllll}1.4192 & 0.0186 & 0.1471 & 0.0018 & 0.0309 & 0.0003 & 1.69\end{array}$ $\begin{array}{llllllll}0.9871 & 0.0151 & 0.1027 & 0.0013 & 0.0269 & 0.0003 & 1.67\end{array}$ $\begin{array}{llllllll}11.5337 & 0.1287 & 0.4876 & 0.0059 & 0.1145 & 0.0009 & 0.95\end{array}$ $\begin{array}{llllllll}1.6507 & 0.0209 & 0.1675 & 0.0020 & 0.0424 & 0.0004 & 2.34\end{array}$ $\begin{array}{lllllll}8.5718 & 0.0970 & 0.4150 & 0.0050 & 0.0836 & 0.0007 & 1.21\end{array}$ $\begin{array}{llllllll}0.1555 & 0.0045 & 0.0228 & 0.0003 & 0.0059 & 0.0001 & 1.01\end{array}$ $\begin{array}{lllllll}1.2861 & 0.0192 & 0.1438 & 0.0018 & 0.0368 & 0.0004 & 2.22\end{array}$ $\begin{array}{llllllll}1.0921 & 0.0148 & 0.1257 & 0.0015 & 0.0291 & 0.0003 & 2.40\end{array}$ $\begin{array}{lllllll}0.6186 & 0.0107 & 0.0780 & 0.0010 & 0.0207 & 0.0003 & 2.12\end{array}$ $\begin{array}{llllllll}0.5178 & 0.0069 & 0.0690 & 0.0008 & 0.0133 & 0.0001 & 2.86\end{array}$ $\begin{array}{lllllll}1.2845 & 0.0282 & 0.1435 & 0.0019 & 0.0396 & 0.0005 & 1.67\end{array}$ $\begin{array}{llllllll}0.6321 & 0.0125 & 0.0831 & 0.0011 & 0.0226 & 0.0002 & 0.83\end{array}$ $\begin{array}{lll}10.0187 & 0.1080 & 0.4539\end{array}$ $\begin{array}{llll}0.0055 & 0.1236 & 0.0012 & 11.38\end{array}$ $\begin{array}{llll}0.0066 & 0.1256 & 0.0012 & 1.40\end{array}$ $\begin{array}{llll}0.0011 & 0.0228 & 0.0002 & 1.83\end{array}$ $\begin{array}{llll}0.0015 & 0.0302 & 0.0005 & 0.72\end{array}$

$\begin{array}{cccccccccc}669 & 13 & 617 & 6 & 582 & 7 & 500 & 4 & 582 & 14 \\ 1534 & 11 & 1496 & 10 & 1423 & 15 & 1178 & 11 & 1534 & 22 \\ 1646 & 12 & 1598 & 11 & 1513 & 16 & 1495 & 13 & 1646 & 24 \\ 2328 & 10 & 2275 & 12 & 2150 & 22 & 1768 & 15 & 2328 & 20 \\ 591 & 35 & 510 & 11 & 475 & 6 & 400 & 4 & 475 & 12 \\ 883 & 15 & 933 & 10 & 923 & 11 & 795 & 8 & 923 & 22 \\ 1530 & 10 & 1546 & 9 & 1509 & 16 & 1376 & 11 & 1530 & 20 \\ 681 & 17 & 577 & 7 & 533 & 6 & 608 & 6 & 533 & 12 \\ 623 & 14 & 586 & 6 & 558 & 6 & 394 & 5 & 558 & 12 \\ 711 & 18 & 569 & 8 & 517 & 6 & 442 & 5 & 517 & 12 \\ 948 & 13 & 1000 & 9 & 991 & 11 & 827 & 7 & 991 & 22 \\ 1187 & 12 & 1164 & 10 & 1116 & 12 & 924 & 8 & 1187 & 24 \\ 1088 & 13 & 1173 & 10 & 1182 & 13 & 884 & 8 & 1088 & 26 \\ 992 & 14 & 1003 & 9 & 977 & 11 & 762 & 7 & 977 & 22 \\ 1440 & 11 & 1477 & 9 & 1457 & 16 & 1018 & 10 & 1440 & 22 \\ 1045 & 19 & 1086 & 13 & 1072 & 13 & 951 & 10 & 1045 & 38 \\ 1000 & 12 & 1061 & 9 & 1057 & 12 & 821 & 8 & 1000 & 24 \\ 1106 & 16 & 1076 & 11 & 1029 & 12 & 592 & 7 & 1106 & 32 \\ 857 & 12 & 897 & 8 & 885 & 10 & 615 & 6 & 885 & 20 \\ 850 & 15 & 697 & 8 & 630 & 7 & 536 & 6 & 630 & 14 \\ 2516 & 9 & 2567 & 10 & 2560 & 25 & 2192 & 16 & 2516 & 18 \\ 902 & 12 & 990 & 8 & 998 & 11 & 839 & 7 & 998 & 22 \\ 2286 & 9 & 2294 & 10 & 2238 & 23 & 1623 & 12 & 2286 & 18 \\ 88 & 44 & 147 & 4 & 145 & 2 & 119 & 2 & 145 & 4 \\ 701 & 14 & 840 & 9 & 866 & 10 & 731 & 8 & 866 & 20 \\ 640 & 13 & 750 & 7 & 763 & 9 & 579 & 6 & 763 & 18 \\ 441 & 18 & 489 & 7 & 484 & 6 & 415 & 5 & 484 & 12 \\ 315 & 13 & 424 & 5 & 430 & 5 & 266 & 3 & 430 & 10 \\ 705 & 25 & 839 & 13 & 864 & 11 & 785 & 10 & 864 & 22 \\ 347 & 23 & 497 & 8 & 515 & 6 & 451 & 4 & 515 & 12 \\ 2404 & 9 & 2437 & 10 & 2412 & 24 & 2356 & 21 & 2404 & 18 \\ 2554 & 9 & 2666 & 11 & 2745 & 28 & 2392 & 21 & 2554 & 18 \\ 214 & 16 & 490 & 6 & 535 & 6 & 455 & 4 & 535 & 12 \\ 565 & 48 & 641 & 16 & 643 & 9 & 602 & 10 & 643 & 18 \\ & & & & & & & & & \end{array}$


$10 \mathrm{~L} 06106$ $10 \mathrm{~L} 06109$

$10 \mathrm{~L} 06112$

10L06115

$10 \mathrm{~L} 06116$ $\begin{array}{lllllllll}0.0568 & 0.0016 & 0.6319 & 0.0155 & 0.0806 & 0.0010 & 0.0251 & 0.0003 & 2.94\end{array}$ $\begin{array}{llllllllll}0.0518 & 0.0012 & 0.6338 & 0.0153 & 0.0862 & 0.0011 & 0.0543 & 0.0035 & 42.20\end{array}$

$\begin{array}{llllllllll}0.0575 & 0.0013 & 0.6958 & 0.0160 & 0.0853 & 0.0011 & 0.0254 & 0.0003 & 1.32\end{array}$

$0.0436 \quad 0.0024$ $0.1602 \quad 0.0015$ 
10L06160 10L06163

10L06165

$10 \mathrm{~L} 06166$

10L06167

10L06168

10L06169

10L06170

10L06171

10L06172

10L06175

$10 \mathrm{~L} 06176$

10L06177

10L06178

10L06179

$10 \mathrm{~L} 06180$

10L06181

10L06182

$10 L 06183$

10L06184

10L06187

10L06189

10L06190

10L06191

10L06192

10L06193

10L06194

10L06195

$10 L 06196$

$10 \mathrm{~L} 06200$

10L06201

$10 L 06202$

10L06203

10L06204 $\begin{array}{ll}0.0477 & 0.0013\end{array}$

$0.0614 \quad 0.0035$

$0.0648 \quad 0.0015$

$0.0577 \quad 0.0015$

$0.0565 \quad 0.0019$

$\begin{array}{ll}0.0461 & 0.0047\end{array}$

$\begin{array}{ll}0.0617 & 0.0010\end{array}$

$\begin{array}{ll}0.0477 & 0.0011\end{array}$

$\begin{array}{ll}0.0567 & 0.0006\end{array}$

$\begin{array}{ll}0.0553 & 0.0007\end{array}$

$0.0682 \quad 0.0008$

$0.0552 \quad 0.0007$

$0.0501 \quad 0.0018$

$0.0662 \quad 0.0009$

$0.0774 \quad 0.0008$

$0.0958 \quad 0.0013$

$0.1091 \quad 0.0011$

$0.0635 \quad 0.0028$

$0.0705 \quad 0.0050$

$0.0652 \quad 0.0011$

$0.0554 \quad 0.0012$

$0.0607 \quad 0.0015$

$0.0667 \quad 0.0011$

$\begin{array}{ll}0.2342 & 0.0028 \\ 0.0806 & 0.0010\end{array}$

$\begin{array}{ll}0.0698 & 0.0009\end{array}$

$\begin{array}{ll}0.0697 & 0.0008 \\ 0.1886 & 0.0020\end{array}$

$0.1846 \quad 0.0020$

$0.0633 \quad 0.0011$

$0.0553 \quad 0.0010$

$0.0571 \quad 0.0016$

$0.0713 \quad 0.0010$

$0.0555 \quad 0.0006$

$0.1886 \quad 0.0024$ $\begin{array}{lllllll}0.2276 & 0.0064 & 0.0335 & 0.0005 & 0.0101 & 0.0002 & 1.46\end{array}$ $\begin{array}{llllllll}0.6601 & 0.0364 & 0.0780 & 0.0012 & 0.0240 & 0.0003 & 0.80\end{array}$ $\begin{array}{llllllll}1.2777 & 0.0305 & 0.1387 & 0.0019 & 0.0407 & 0.0004 & 0.29\end{array}$ $\begin{array}{llllllll}0.8513 & 0.0228 & 0.1037 & 0.0014 & 0.0312 & 0.0005 & 1.96\end{array}$ $\begin{array}{llllllll}0.6866 & 0.0232 & 0.0855 & 0.0012 & 0.0256 & 0.0008 & 3.32\end{array}$ $\begin{array}{lllllll}0.1441 & 0.0144 & 0.0227 & 0.0004 & 0.0073 & 0.0004 & 1.81\end{array}$ $\begin{array}{lllllll}0.6842 & 0.0122 & 0.0779 & 0.0010 & 0.0219 & 0.0003 & 2.18\end{array}$ $\begin{array}{llllllll}0.1969 & 0.0048 & 0.0290 & 0.0004 & 0.0086 & 0.0001 & 1.10\end{array}$ $\begin{array}{llllllll}0.6617 & 0.0088 & 0.0820 & 0.0010 & 0.0206 & 0.0002 & 4.12\end{array}$ $\begin{array}{lllllll}0.6657 & 0.0100 & 0.0845 & 0.0011 & 0.0246 & 0.0002 & 1.73\end{array}$ $\begin{array}{llllllll}1.3638 & 0.0184 & 0.1406 & 0.0018 & 0.0386 & 0.0004 & 2.40\end{array}$ $\begin{array}{lllllll}0.6543 & 0.0096 & 0.0833 & 0.0010 & 0.0254 & 0.0003 & 1.90\end{array}$ $\begin{array}{llllllll}0.0991 & 0.0035 & 0.0139 & 0.0002 & 0.0058 & 0.0002 & 5.20\end{array}$ $\begin{array}{lllllll}1.3432 & 0.0197 & 0.1426 & 0.0018 & 0.0386 & 0.0004 & 2.23\end{array}$ $\begin{array}{llllllll}2.1155 & 0.0269 & 0.1921 & 0.0024 & 0.0514 & 0.0004 & 1.36\end{array}$ $\begin{array}{lllllll}3.4820 & 0.0529 & 0.2555 & 0.0033 & 0.0726 & 0.0008 & 1.47\end{array}$ $\begin{array}{lllllll}4.9612 & 0.0614 & 0.3196 & 0.0040 & 0.0884 & 0.0008 & 2.59\end{array}$ $\begin{array}{llllllll}0.8270 & 0.0337 & 0.0944 & 0.0014 & 0.0290 & 0.0004 & 2.38\end{array}$ $\begin{array}{llllllll}1.3512 & 0.0940 & 0.1348 & 0.0027 & 0.0390 & 0.0010 & 0.69\end{array}$ $\begin{array}{llllllll}1.3099 & 0.0240 & 0.1411 & 0.0018 & 0.0377 & 0.0004 & 0.69\end{array}$ $\begin{array}{llllllll}0.6395 & 0.0140 & 0.0812 & 0.0011 & 0.0218 & 0.0002 & 0.74\end{array}$ $\begin{array}{llllllll}0.8141 & 0.0201 & 0.0943 & 0.0013 & 0.0246 & 0.0003 & 0.36\end{array}$ $\begin{array}{llllllll}1.2242 & 0.0217 & 0.1290 & 0.0017 & 0.0357 & 0.0004 & 1.04\end{array}$ $\begin{array}{llllllll}20.8429 & 0.2883 & 0.6256 & 0.0081 & 0.1427 & 0.0017 & 1.42\end{array}$ $\begin{array}{llllllll}2.3712 & 0.0344 & 0.2067 & 0.0026 & 0.0531 & 0.0005 & 1.23\end{array}$ $\begin{array}{llllllll}1.4347 & 0.0210 & 0.1445 & 0.0018 & 0.0394 & 0.0005 & 3.31\end{array}$ $\begin{array}{lllllll}1.6190 & 0.0222 & 0.1632 & 0.0020 & 0.0496 & 0.0006 & 5.93\end{array}$ $\begin{array}{lllllll}13.6308 & 0.1760 & 0.5190 & 0.0066 & 0.1223 & 0.0012 & 1.38\end{array}$ $\begin{array}{lllllll}1.1483 & 0.0216 & 0.1274 & 0.0017 & 0.0324 & 0.0004 & 1.50\end{array}$ $\begin{array}{llllllll}0.6452 & 0.0123 & 0.0820 & 0.0011 & 0.0219 & 0.0003 & 2.37\end{array}$ $\begin{array}{llllllll}0.7429 & 0.0208 & 0.0915 & 0.0013 & 0.0244 & 0.0003 & 0.80\end{array}$ $\begin{array}{lllllll}1.7511 & 0.0270 & 0.1725 & 0.0022 & 0.0444 & 0.0004 & 0.44\end{array}$ $\begin{array}{lllllll}0.6516 & 0.0086 & 0.0825 & 0.0010 & 0.0221 & 0.0002 & 1.75\end{array}$ $\begin{array}{lllllll}13.0653 & 0.1898 & 0.4869 & 0.0064 & 0.1204 & 0.0012 & 0.60\end{array}$

$\begin{array}{cccccccccc}85 & 41 & 208 & 5 & 213 & 3 & 204 & 3 & 213 & 6 \\ 653 & 126 & 515 & 22 & 484 & 7 & 480 & 5 & 484 & 14 \\ 767 & 28 & 836 & 14 & 837 & 11 & 806 & 8 & 837 & 22 \\ 520 & 35 & 625 & 12 & 636 & 8 & 621 & 11 & 636 & 16 \\ 471 & 50 & 531 & 14 & 529 & 7 & 510 & 15 & 529 & 14 \\ & 208 & 137 & 13 & 145 & 3 & 147 & 9 & 145 & 6 \\ 665 & 18 & 529 & 7 & 484 & 6 & 437 & 6 & 484 & 12 \\ 85 & 34 & 182 & 4 & 184 & 2 & 173 & 2 & 184 & 4 \\ 481 & 13 & 516 & 5 & 508 & 6 & 412 & 4 & 508 & 12 \\ 426 & 15 & 518 & 6 & 523 & 6 & 490 & 5 & 523 & 12 \\ 874 & 12 & 873 & 8 & 848 & 10 & 765 & 7 & 848 & 20 \\ 421 & 15 & 511 & 6 & 516 & 6 & 506 & 5 & 516 & 12 \\ 197 & 54 & 96 & 3 & 89 & 1 & 117 & 3 & 89 & 2 \\ 813 & 14 & 865 & 9 & 859 & 10 & 766 & 8 & 859 & 20 \\ 1132 & 11 & 1154 & 9 & 1133 & 13 & 1013 & 8 & 1132 & 22 \\ 1544 & 13 & 1523 & 12 & 1467 & 17 & 1417 & 15 & 1544 & 26 \\ 1785 & 10 & 1813 & 10 & 1788 & 19 & 1712 & 15 & 1785 & 20 \\ 726 & 94 & 612 & 19 & 582 & 8 & 577 & 7 & 582 & 16 \\ 942 & 110 & 868 & 41 & 815 & 15 & 773 & 20 & 815 & 30 \\ 782 & 19 & 850 & 11 & 851 & 10 & 747 & 7 & 851 & 20 \\ 428 & 27 & 502 & 9 & 503 & 6 & 436 & 5 & 503 & 12 \\ 628 & 31 & 605 & 11 & 581 & 7 & 492 & 5 & 581 & 14 \\ 828 & 18 & 812 & 10 & 782 & 9 & 710 & 7 & 782 & 18 \\ 3081 & 10 & 3131 & 13 & 3132 & 32 & 2695 & 31 & 3081 & 20 \\ 1212 & 13 & 1234 & 10 & 1211 & 14 & 1046 & 10 & 1212 & 26 \\ 921 & 13 & 904 & 9 & 870 & 10 & 781 & 9 & 870 & 20 \\ 920 & 13 & 978 & 9 & 975 & 11 & 979 & 11 & 975 & 22 \\ 2694 & 10 & 2724 & 12 & 2695 & 28 & 2331 & 21 & 2694 & 20 \\ 719 & 20 & 776 & 10 & 773 & 9 & 645 & 8 & 773 & 18 \\ 424 & 21 & 506 & 8 & 508 & 6 & 438 & 6 & 508 & 12 \\ 494 & 38 & 564 & 12 & 564 & 7 & 487 & 6 & 564 & 14 \\ 967 & 14 & 1028 & 10 & 1026 & 12 & 879 & 7 & 1026 & 24 \\ 434 & 13 & 509 & 5 & 511 & 6 & 441 & 4 & 511 & 12 \\ 2730 & 11 & 2684 & 14 & 2557 & 28 & 2298 & 21 & 2730 & 22 \\ & & & & & & & & & \end{array}$




\begin{tabular}{|c|c|c|c|c|c|c|c|c|c|c|c|c|c|c|c|c|c|c|c|}
\hline 10L06205 & 0.1503 & 0.0015 & 8.3156 & 0.1008 & 0.3887 & 0.0048 & 0.0586 & 0.0005 & 1.96 & 2350 & 10 & 2266 & 11 & 2117 & 22 & 1150 & 10 & 2350 & 20 \\
\hline 10L06206 & 0.0720 & 0.0009 & 1.6270 & 0.0229 & 0.1588 & 0.0020 & 0.0381 & 0.0004 & 2.69 & 987 & 13 & 981 & 9 & 950 & 11 & 756 & 8 & 950 & 22 \\
\hline L06208 & 0.0688 & 0.0008 & 1.5626 & 0.0220 & 0.1597 & 0.0020 & 0.0421 & 0.0004 & 2.00 & 891 & 13 & 956 & 9 & 955 & 11 & 833 & 8 & 55 & 22 \\
\hline 10L06211 & 0.0531 & 0.0011 & 0.3542 & 0.0077 & 0.0468 & 0.0006 & 0.0130 & 0.0002 & 1.46 & 335 & 27 & 308 & 6 & 295 & 4 & 261 & 3 & 295 & 8 \\
\hline OL06212 & 0.0579 & 0.0007 & 0.7259 & 0.0104 & 0.0881 & 0.0011 & 0.0235 & 0.0002 & 2.84 & 526 & 14 & 554 & 6 & 544 & 7 & 470 & 5 & 544 & 14 \\
\hline LL06213 & 0.0567 & 0.0012 & 0.6494 & 0.0142 & 0.0806 & 0.0011 & 0.0217 & 0.0003 & 0.96 & 478 & 26 & 508 & 9 & 499 & 6 & 433 & 5 & 499 & 2 \\
\hline 10L06214 & 0.0556 & 0.0008 & 0.6485 & 0.0100 & 0.0819 & 0.0010 & 0.0221 & 0.0002 & 2.49 & 437 & 16 & 508 & 6 & 508 & 6 & 443 & 5 & 508 & 2 \\
\hline OL06215 & 0.0588 & 0.0010 & 0.7561 & 0.0140 & 0.0903 & 0.0012 & 0.0242 & 0.0002 & 0.49 & 561 & 20 & 572 & 8 & 557 & 7 & 484 & 4 & 557 & 14 \\
\hline OL06216 & 0.0745 & 0.0012 & 1.9072 & 0.0337 & 0.1799 & 0.0023 & 0.0476 & 0.0005 & 0.88 & 1056 & 17 & 1084 & 12 & 1066 & 13 & 940 & 9 & 1056 & 34 \\
\hline OL06217 & 0.0666 & 0.0008 & 1.3890 & 0.0198 & 0.1465 & 0.0018 & 0.0388 & 0.0004 & 2.63 & 826 & 13 & 884 & 8 & 882 & 10 & 770 & 8 & 882 & 0 \\
\hline OL06218 & 0.0707 & 0.0012 & 1.7425 & 0.0312 & 0.1732 & 0.0022 & 0.0445 & 0.0005 & 0.94 & 948 & 18 & 1024 & 12 & 1030 & 12 & 880 & 9 & 1030 & 24 \\
\hline OL06219 & 0.1819 & 0.0018 & 11.9130 & 0.1474 & 0.4602 & 0.0057 & 0.1097 & 0.0009 & 1.87 & 2670 & 9 & 2598 & 12 & 2441 & 25 & 2104 & 17 & 2670 & 8 \\
\hline JL06224 & 0.0581 & 0.0009 & 0.7915 & 0.0139 & 0.0958 & 0.0012 & 0.0278 & 0.0003 & 2.15 & 532 & 19 & 592 & 8 & 590 & 7 & 553 & 6 & 590 & 14 \\
\hline 10L06225 & 0.0884 & 0.0017 & 3.0270 & 0.0596 & 0.2407 & 0.0032 & 0.0679 & 0.0009 & 1.57 & 1390 & 19 & 1414 & 15 & 1390 & 17 & 1328 & 18 & 1390 & 38 \\
\hline OL06226 & 0.1012 & 0.0012 & 4.1957 & 0.0571 & 0.2914 & 0.0037 & 0.0785 & 0.0007 & 1.12 & 1646 & 11 & 1673 & 11 & 1649 & 18 & 1528 & 13 & 1646 & 22 \\
\hline OLOC & 0.0 & 0.0014 & 2.3721 & 0.0 & 0.2002 & & 51 & 0.0005 & 0.65 & 1276 & 16 & 1234 & 13 & 1176 & 14 & 1084 & 10 & 1276 & 32 \\
\hline OL06228 & 0.0570 & 0.0018 & 0.6620 & 0.0215 & 0.0817 & 0.0012 & 0.0231 & 0.0003 & 0.78 & 490 & 47 & 516 & 13 & 506 & 7 & 461 & 6 & 506 & 14 \\
\hline 10L06229 & 0.0584 & 0.0019 & 0.8292 & 0.0266 & 0.0998 & 0.0014 & 0.0279 & 0.0004 & 0.76 & 545 & 45 & 613 & 15 & 613 & 8 & 556 & 7 & 613 & 16 \\
\hline OLOG & 0.0714 & 0.0013 & 1.5445 & 0.0289 & 0.1520 & 20 & 22 & 0.0005 & 1.28 & 969 & 19 & 948 & 12 & 912 & 11 & 836 & 9 & 912 & 22 \\
\hline 10L06231 & 0.0843 & 0.0010 & 2.6763 & 0.0379 & 0.2231 & 0.0028 & 0.0639 & 0.0006 & 1.73 & 1299 & 12 & 1322 & 10 & 1298 & 15 & 1251 & 11 & 1299 & 24 \\
\hline 10L06235 & 0.0694 & 0.0016 & 1.1237 & 0.0221 & 0.1175 & 0.0015 & 0.0357 & 0.0004 & 4.99 & 910 & 49 & 765 & 11 & 716 & 9 & 709 & 9 & 716 & 18 \\
\hline 10L06236 & 0.0775 & 0.0011 & 1.8244 & 0.0296 & 0.1654 & 0.0021 & 0.0418 & 0.0004 & 0.96 & 1134 & 15 & 1054 & 11 & 987 & 12 & 827 & 8 & 987 & 24 \\
\hline 10L06237 & 0.0760 & 0.0012 & 2.0708 & 0.0348 & 0.1915 & 0.0025 & 0.0553 & 0.0005 & 0.83 & 1094 & 16 & 1139 & 11 & 1130 & 13 & 1088 & 10 & 1094 & 32 \\
\hline 10L06238 & 0.0582 & 0.0009 & 0.7361 & 0.0123 & 0.0888 & 0.0011 & 0.0270 & 0.0004 & 7.85 & 539 & 17 & 560 & 7 & 548 & 7 & 538 & 9 & 548 & 14 \\
\hline 10L06239 & 0.0650 & 0.0008 & 1.2376 & 0.0178 & 0.1337 & 0.0017 & 0.0392 & 0.0004 & 1.52 & 776 & 14 & 818 & 8 & 809 & 10 & 777 & 7 & 809 & 20 \\
\hline 10L06240 & 0.0666 & 0.0020 & 1.0898 & 0.0284 & 0.1187 & 0.0016 & 0.0362 & 0.0004 & 1.91 & 826 & 62 & 748 & 14 & 723 & 9 & 719 & 8 & 723 & 18 \\
\hline 10L06241 & 0.0667 & 0.0010 & 1.4228 & 0.0242 & 0.1498 & 0.0019 & 0.0452 & 0.0006 & 3.83 & 829 & 17 & 899 & 10 & 900 & 11 & 894 & 12 & 900 & 22 \\
\hline 10L06242 & 0.0766 & 0.0017 & 2.0378 & 0.0455 & 0.1869 & 0.0025 & 0.0558 & 0.0006 & 0.75 & 1111 & 24 & 1128 & 15 & 1105 & 14 & 1097 & 12 & 1111 & 48 \\
\hline 10L06243 & 0.0580 & 0.0016 & 0.7202 & 0.0198 & 0.0873 & 0.0012 & 0.0269 & 0.0003 & 0.30 & 529 & 36 & 551 & 12 & 539 & 7 & 537 & 5 & 539 & 14 \\
\hline 10L06244 & 0.0797 & 0.0014 & 2.1583 & 0.0402 & 0.1902 & 0.0025 & 0.0579 & 0.0006 & 1.02 & 1190 & 18 & 1168 & 13 & 1123 & 13 & 1137 & 12 & 1190 & 36 \\
\hline
\end{tabular}

2009TL1166 (Liuqu, upper part of the Za section, $\mathrm{n}=72, \mathrm{~N}^{\circ} 9^{\circ} 09.812^{\prime} \quad \mathrm{E88^{ \circ }} 08.103^{\prime}$, $4341 \mathrm{~m}$ )

$\begin{array}{llllllllllllllllllll}\text { 1OL01004 } & 0.0621 & 0.0008 & 1.4520 & 0.0185 & 0.1646 & 0.0018 & 0.0431 & 0.0003 & 1.00 & 676 & 12 & 911 & 8 & 982 & 10 & 852 & 7 & 982 & 20\end{array}$ $\begin{array}{llllllllllllllllllllll}\text { 10L01005 } & 0.0575 & 0.0011 & 0.7567 & 0.0148 & 0.0926 & 0.0011 & 0.0256 & 0.0002 & 0.63 & 510 & 24 & 572 & 9 & 571 & 6 & 511 & 5 & 571 & 12\end{array}$ 
10L01006 10L01007

10L01008

10L01009

10L01010

10L01011

10L01012

10L01016

10L01019

$10 \mathrm{~L} 01020$

10L01021

$10 L 01022$

10L01023

10L01024

10L01025

$10 L 01030$

10L01031

10L01032

10L01033

$10 L 01034$

10L01035

$10 \mathrm{~L} 01036$

10L01037

10L01041

10L01044

$10 \mathrm{~L} 01046$

10L01048

10L01049

10L01052

10L01053

10L01054

10L01055

10L01058

10L01059 $\begin{array}{lllllllll}0.0978 & 0.0015 & 3.8703 & 0.0584 & 0.2784 & 0.0032 & 0.0723 & 0.0006 & 0.55\end{array}$ $0.0711 \quad 0.0010$

$\begin{array}{ll}0.0616 & 0.0007\end{array}$

$0.0688 \quad 0.0007$

$0.0935 \quad 0.0010$

$\begin{array}{lll}0.0607 & 0.0008\end{array}$

$0.1248 \quad 0.0012$

$0.0722 \quad 0.0011$

$\begin{array}{lll}0.0564 & 0.0009\end{array}$

$0.0598 \quad 0.0008$

$0.0736 \quad 0.0010$

$\begin{array}{ll}0.0673 & 0.0007\end{array}$

$0.0468 \quad 0.0030$

$\begin{array}{ll}0.0546 & 0.0007\end{array}$

$0.1393 \quad 0.0013$

$0.0781 \quad 0.0010$

$0.0523 \quad 0.0008$

$0.0552 \quad 0.0008$

$0.0620 \quad 0.0010$

$0.0540 \quad 0.0007$

$0.0622 \quad 0.0007$

$\begin{array}{ll}0.0662 & 0.0009\end{array}$

$0.0885 \quad 0.0011$

$\begin{array}{ll}0.0806 & 0.0009\end{array}$

$0.0557 \quad 0.0008$

$\begin{array}{ll}0.1713 & 0.0019 \\ 0.0675 & 0.0008 \\ 0.0649 & 0.0007\end{array}$

$\begin{array}{ll}0.0649 & 0.0007 \\ 0.0791 & 0.0009\end{array}$

$\begin{array}{ll}0.0791 & 0.0009\end{array}$

$0.0713 \quad 0.0011$

$\begin{array}{ll}0.0577 & 0.0007\end{array}$

$0.0845 \quad 0.0010$

$0.0698 \quad 0.0009$

$0.0717 \quad 0.0009$

$\begin{array}{lllllll}1.7308 & 0.0238 & 0.1712 & 0.0019 & 0.0485 & 0.0005 & 1.60\end{array}$

$\begin{array}{llllllll}1.1152 & 0.0126 & 0.1273 & 0.0014 & 0.0351 & 0.0003 & 1.38\end{array}$

$\begin{array}{lllllll}1.3432 & 0.0150 & 0.1373 & 0.0015 & 0.0256 & 0.0002 & 1.25\end{array}$

$\begin{array}{llllllll}3.4571 & 0.0368 & 0.2602 & 0.0028 & 0.0624 & 0.0005 & 1.58\end{array}$

$\begin{array}{llllllll}0.8987 & 0.0126 & 0.1042 & 0.0011 & 0.0200 & 0.0003 & 2.86\end{array}$

$\begin{array}{llllllll}6.1641 & 0.0644 & 0.3475 & 0.0037 & 0.0664 & 0.0006 & 2.23\end{array}$

$\begin{array}{lllllll}1.7200 & 0.0262 & 0.1676 & 0.0019 & 0.0455 & 0.0004 & 0.61\end{array}$

$\begin{array}{lllllll}0.6928 & 0.0114 & 0.0865 & 0.0010 & 0.0244 & 0.0003 & 1.57\end{array}$

$\begin{array}{lllllll}0.7061 & 0.0100 & 0.0832 & 0.0009 & 0.0214 & 0.0002 & 2.24\end{array}$

$\begin{array}{llllllll}1.9022 & 0.0261 & 0.1818 & 0.0020 & 0.0491 & 0.0004 & 1.12\end{array}$

$\begin{array}{lllllll}1.4470 & 0.0155 & 0.1514 & 0.0016 & 0.0418 & 0.0004 & 4.50\end{array}$

$\begin{array}{llllllll}0.0903 & 0.0057 & 0.0136 & 0.0002 & 0.0036 & 0.0001 & 1.20\end{array}$

$\begin{array}{llllllll}0.5940 & 0.0079 & 0.0766 & 0.0008 & 0.0220 & 0.0002 & 2.39\end{array}$

$\begin{array}{lllllll}7.8648 & 0.0805 & 0.3975 & 0.0043 & 0.0935 & 0.0007 & 1.52\end{array}$

$\begin{array}{llllllll}2.0110 & 0.0273 & 0.1814 & 0.0020 & 0.0508 & 0.0005 & 1.51\end{array}$

$\begin{array}{lllllll}0.4138 & 0.0067 & 0.0557 & 0.0006 & 0.0153 & 0.0001 & 1.11\end{array}$

$\begin{array}{llllllll}0.6225 & 0.0087 & 0.0794 & 0.0009 & 0.0228 & 0.0002 & 2.01\end{array}$

$\begin{array}{lllllll}0.9761 & 0.0154 & 0.1109 & 0.0013 & 0.0348 & 0.0005 & 4.02\end{array}$

$\begin{array}{llllllll}0.6596 & 0.0084 & 0.0861 & 0.0010 & 0.0335 & 0.0005 & 14.09\end{array}$

$\begin{array}{lllllll}1.2313 & 0.0150 & 0.1395 & 0.0015 & 0.0386 & 0.0004 & 2.61\end{array}$

$\begin{array}{llllllll}1.3272 & 0.0184 & 0.1413 & 0.0016 & 0.0379 & 0.0003 & 0.55\end{array}$

$\begin{array}{llllllll}3.0381 & 0.0400 & 0.2418 & 0.0027 & 0.0617 & 0.0005 & 0.66\end{array}$

$\begin{array}{llllllll}2.1677 & 0.0252 & 0.1895 & 0.0021 & 0.0396 & 0.0005 & 6.27\end{array}$

$\begin{array}{llllllll}0.6257 & 0.0089 & 0.0792 & 0.0009 & 0.0341 & 0.0006 & 13.43\end{array}$

$\begin{array}{llllllll}11.4359 & 0.1364 & 0.4704 & 0.0054 & 0.1112 & 0.0011 & 1.07\end{array}$

$\begin{array}{lllllll}1.1952 & 0.0149 & 0.1248 & 0.0014 & 0.0353 & 0.0003 & 1.75\end{array}$

$\begin{array}{llllllll}1.3543 & 0.0159 & 0.1470 & 0.0016 & 0.0389 & 0.0003 & 2.29\end{array}$

$\begin{array}{lllllll}2.0047 & 0.0246 & 0.1786 & 0.0020 & 0.0504 & 0.0005 & 2.18\end{array}$

$\begin{array}{lllllll}1.6904 & 0.0273 & 0.1670 & 0.0020 & 0.0473 & 0.0005 & 1.06\end{array}$

$\begin{array}{llllllll}0.6560 & 0.0084 & 0.0800 & 0.0009 & 0.0222 & 0.0002 & 2.56\end{array}$

$\begin{array}{lllllll}2.7068 & 0.0330 & 0.2256 & 0.0026 & 0.0621 & 0.0005 & 1.35\end{array}$

$\begin{array}{llllllll}1.6161 & 0.0210 & 0.1631 & 0.0019 & 0.0465 & 0.0004 & 1.94\end{array}$

$\begin{array}{lllllll}1.7202 & 0.0224 & 0.1690 & 0.0019 & 0.0505 & 0.0005 & 1.90\end{array}$

$\begin{array}{cccccccccc}1582 & 13 & 1608 & 12 & 1583 & 16 & 1410 & 12 & 1582 & 26 \\ 961 & 13 & 1020 & 9 & 1019 & 10 & 957 & 9 & 961 & 26 \\ 661 & 11 & 761 & 6 & 773 & 8 & 698 & 5 & 773 & 16 \\ 893 & 10 & 865 & 7 & 830 & 8 & 510 & 4 & 830 & 16 \\ 1497 & 9 & 1517 & 8 & 1491 & 14 & 1224 & 9 & 1497 & 18 \\ 628 & 14 & 651 & 7 & 639 & 7 & 400 & 5 & 639 & 14 \\ 2026 & 9 & 1999 & 9 & 1923 & 18 & 1299 & 11 & 2026 & 18 \\ 992 & 15 & 1016 & 10 & 999 & 10 & 900 & 7 & 999 & 20 \\ 467 & 18 & 535 & 7 & 535 & 6 & 487 & 5 & 535 & 12 \\ 595 & 14 & 542 & 6 & 515 & 5 & 428 & 5 & 515 & 10 \\ 1032 & 13 & 1082 & 9 & 1077 & 11 & 968 & 8 & 1032 & 26 \\ 847 & 10 & 909 & 6 & 909 & 9 & 828 & 7 & 909 & 18 \\ 41 & 107 & 88 & 5 & 87 & 1 & 72 & 2 & 87 & 2 \\ 395 & 13 & 473 & 5 & 476 & 5 & 440 & 4 & 476 & 10 \\ 2219 & 8 & 2216 & 9 & 2157 & 20 & 1807 & 13 & 2219 & 16 \\ 1148 & 12 & 1119 & 9 & 1075 & 11 & 1001 & 9 & 1148 & 24 \\ 300 & 18 & 352 & 5 & 349 & 4 & 307 & 3 & 349 & 8 \\ 421 & 14 & 491 & 5 & 492 & 5 & 456 & 4 & 492 & 10 \\ 673 & 16 & 692 & 8 & 678 & 7 & 692 & 10 & 678 & 14 \\ 369 & 13 & 514 & 5 & 532 & 6 & 666 & 10 & 532 & 12 \\ 680 & 12 & 815 & 7 & 842 & 9 & 765 & 7 & 842 & 18 \\ 811 & 13 & 858 & 8 & 852 & 9 & 753 & 6 & 852 & 18 \\ 1393 & 11 & 1417 & 10 & 1396 & 14 & 1211 & 10 & 1393 & 22 \\ 1211 & 10 & 1171 & 8 & 1119 & 11 & 785 & 10 & 1211 & 20 \\ 439 & 15 & 493 & 6 & 491 & 5 & 677 & 12 & 491 & 10 \\ 2570 & 9 & 2559 & 11 & 2485 & 24 & 2131 & 19 & 2570 & 18 \\ 852 & 12 & 798 & 7 & 758 & 8 & 701 & 6 & 758 & 16 \\ 771 & 11 & 869 & 7 & 884 & 9 & 772 & 7 & 884 & 18 \\ 1174 & 11 & 1117 & 8 & 1059 & 11 & 993 & 9 & 1174 & 22 \\ 966 & 16 & 1005 & 10 & 995 & 11 & 933 & 9 & 995 & 22 \\ 520 & 13 & 512 & 5 & 496 & 5 & 444 & 4 & 496 & 10 \\ 1305 & 11 & 1330 & 9 & 1311 & 13 & 1218 & 10 & 1305 & 22 \\ 923 & 12 & 976 & 8 & 974 & 10 & 919 & 8 & 974 & 20 \\ 978 & 12 & 1016 & 8 & 1006 & 11 & 997 & 9 & 978 & 24 \\ & & & & & & & & & \end{array}$


10L01060 10L01061

$10 \mathrm{~L} 01066$

10L01068

10L01069

10L01070

10L01071

10L01076

$10 \mathrm{~L} 01080$

10L01081

$10 \mathrm{~L} 01082$

10L01084

10L01085

$10 \mathrm{~L} 01088$

10L01089

$10 L 01094$

$10 \mathrm{~L} 01095$

10L01096

$10\llcorner 01097$

$10 \mathrm{~L} 01100$

10L01101

$10 \mathrm{~L} 01102$

10L01103

10L01104

10L01108

10L01109

10L01112

10L01113

10L01114

10L01115

$10 \mathrm{~L} 01116$

$10 L 01117$

$10 L 01118$

10L01119 $\begin{array}{ll}0.0560 & 0.0007\end{array}$

$0.1004 \quad 0.0013$

$0.0628 \quad 0.0008$

$\begin{array}{ll}0.1113 & 0.0019\end{array}$

$0.0796 \quad 0.0012$

$0.1095 \quad 0.0012$

$0.0852 \quad 0.0011$

$0.0688 \quad 0.0010$

$\begin{array}{ll}0.1715 & 0.0020\end{array}$

$\begin{array}{ll}0.0662 & 0.0007\end{array}$

$\begin{array}{ll}0.0825 & 0.0011\end{array}$

$0.0642 \quad 0.0012$

$\begin{array}{ll}0.0641 & 0.0009\end{array}$

$0.1448 \quad 0.0014$

$\begin{array}{ll}0.0766 & 0.0012\end{array}$

$0.0569 \quad 0.0013$

$0.0699 \quad 0.0008$

$0.1009 \quad 0.0015$

$0.0724 \quad 0.0009$

$0.0571 \quad 0.0013$

$0.0862 \quad 0.0010$

$0.0686 \quad 0.0011$

$0.0727 \quad 0.0010$

$0.0512 \quad 0.0031$

$0.0697 \quad 0.0008$

$0.0700 \quad 0.0027$

$0.0609 \quad 0.0009$

$\begin{array}{ll}0.0636 & 0.0015\end{array}$

$0.0972 \quad 0.0013$

$\begin{array}{ll}0.0625 & 0.0017\end{array}$

$0.0962 \quad 0.0011$

$0.0652 \quad 0.0008$

$0.0665 \quad 0.0017$

$0.0729 \quad 0.0008$ $\begin{array}{lllllll}0.6420 & 0.0090 & 0.0808 & 0.0009 & 0.0254 & 0.0003 & 3.54\end{array}$ $\begin{array}{lllllll}4.1723 & 0.0574 & 0.2928 & 0.0034 & 0.0804 & 0.0008 & 1.24\end{array}$ $\begin{array}{llllllll}1.0982 & 0.0141 & 0.1232 & 0.0014 & 0.0387 & 0.0004 & 2.72\end{array}$ $\begin{array}{llllllll}4.6855 & 0.0587 & 0.3053 & 0.0035 & 0.0882 & 0.0010 & 4.81\end{array}$ $\begin{array}{llllllll}2.2874 & 0.0351 & 0.2025 & 0.0024 & 0.0640 & 0.0006 & 1.03\end{array}$ $\begin{array}{lllllll}4.7116 & 0.0568 & 0.3031 & 0.0035 & 0.1013 & 0.0009 & 1.22\end{array}$ $\begin{array}{lllllll}2.7566 & 0.0372 & 0.2278 & 0.0027 & 0.0708 & 0.0007 & 1.30\end{array}$ $\begin{array}{lllllll}1.3910 & 0.0210 & 0.1423 & 0.0017 & 0.0423 & 0.0004 & 1.50\end{array}$ $\begin{array}{llllllll}11.9538 & 0.1509 & 0.4908 & 0.0059 & 0.1341 & 0.0014 & 1.12\end{array}$ $\begin{array}{llllllll}1.4697 & 0.0181 & 0.1564 & 0.0018 & 0.0501 & 0.0005 & 3.83\end{array}$ $\begin{array}{lllllll}2.5409 & 0.0363 & 0.2168 & 0.0026 & 0.0580 & 0.0006 & 1.26\end{array}$ $\begin{array}{llllllll}1.1233 & 0.0216 & 0.1232 & 0.0015 & 0.0384 & 0.0005 & 1.47\end{array}$ $\begin{array}{lllllll}0.8832 & 0.0128 & 0.0970 & 0.0012 & 0.0303 & 0.0004 & 5.63\end{array}$ $\begin{array}{llllllll}7.9619 & 0.0872 & 0.3871 & 0.0045 & 0.1072 & 0.0009 & 1.63\end{array}$ $\begin{array}{llllllll}2.1814 & 0.0349 & 0.2006 & 0.0024 & 0.0656 & 0.0008 & 2.21\end{array}$ $\begin{array}{llllllll}0.6792 & 0.0158 & 0.0841 & 0.0011 & 0.0279 & 0.0004 & 1.21\end{array}$ $\begin{array}{llllllll}1.6382 & 0.0214 & 0.1650 & 0.0020 & 0.0569 & 0.0006 & 4.09\end{array}$ $\begin{array}{llllllll}4.1470 & 0.0637 & 0.2893 & 0.0036 & 0.0915 & 0.0009 & 0.84\end{array}$ $\begin{array}{lllllll}1.7512 & 0.0243 & 0.1702 & 0.0020 & 0.0552 & 0.0005 & 1.28\end{array}$ $\begin{array}{llllllll}0.7588 & 0.0178 & 0.0934 & 0.0012 & 0.0299 & 0.0003 & 0.33\end{array}$ $\begin{array}{llllllll}2.8403 & 0.0377 & 0.2317 & 0.0028 & 0.0779 & 0.0007 & 1.48\end{array}$ $\begin{array}{llllllll}1.5679 & 0.0272 & 0.1608 & 0.0020 & 0.0527 & 0.0005 & 0.89\end{array}$ $\begin{array}{llllllll}1.7484 & 0.0257 & 0.1690 & 0.0020 & 0.0606 & 0.0007 & 2.24\end{array}$ $\begin{array}{llllllll}0.1264 & 0.0075 & 0.0173 & 0.0003 & 0.0061 & 0.0002 & 1.21\end{array}$ $\begin{array}{lllllll}1.6306 & 0.0204 & 0.1645 & 0.0020 & 0.0875 & 0.0008 & 2.08\end{array}$ $\begin{array}{llllllll}1.2669 & 0.0479 & 0.1272 & 0.0019 & 0.0447 & 0.0008 & 0.90\end{array}$ $\begin{array}{llllllll}0.9862 & 0.0149 & 0.1138 & 0.0014 & 0.0392 & 0.0005 & 2.76\end{array}$ $\begin{array}{llllllll}0.8677 & 0.0211 & 0.0959 & 0.0013 & 0.0386 & 0.0007 & 2.28\end{array}$ $\begin{array}{lllllll}3.9309 & 0.0565 & 0.2841 & 0.0035 & 0.0921 & 0.0010 & 1.65\end{array}$ $\begin{array}{lllllll}0.7830 & 0.0209 & 0.0880 & 0.0012 & 0.0324 & 0.0005 & 1.27\end{array}$ $\begin{array}{llllllll}3.7707 & 0.0494 & 0.2754 & 0.0034 & 0.0988 & 0.0009 & 0.88\end{array}$ $\begin{array}{llllllll}1.1734 & 0.0159 & 0.1263 & 0.0015 & 0.0360 & 0.0003 & 0.67\end{array}$ $\begin{array}{llllllll}1.1639 & 0.0266 & 0.1270 & 0.0016 & 0.0388 & 0.0005 & 2.37\end{array}$ $\begin{array}{lllllll}1.9003 & 0.0238 & 0.1831 & 0.0022 & 0.0579 & 0.0005 & 1.50\end{array}$

$\begin{array}{cccccccccc}451 & 14 & 504 & 6 & 501 & 5 & 506 & 6 & 501 & 10 \\ 1631 & 11 & 1669 & 11 & 1656 & 17 & 1562 & 15 & 1631 & 22 \\ 701 & 12 & 752 & 7 & 749 & 8 & 767 & 7 & 749 & 16 \\ 1821 & 31 & 1765 & 10 & 1717 & 17 & 1708 & 19 & 1821 & 62 \\ 1186 & 14 & 1208 & 11 & 1189 & 13 & 1254 & 12 & 1186 & 28 \\ 1791 & 10 & 1769 & 10 & 1707 & 17 & 1951 & 16 & 1791 & 20 \\ 1321 & 12 & 1344 & 10 & 1323 & 14 & 1382 & 13 & 1321 & 24 \\ 894 & 14 & 885 & 9 & 858 & 9 & 838 & 9 & 858 & 18 \\ 2572 & 9 & 2601 & 12 & 2574 & 25 & 2544 & 24 & 2572 & 18 \\ 811 & 12 & 918 & 7 & 937 & 10 & 988 & 10 & 937 & 20 \\ 1258 & 13 & 1284 & 10 & 1265 & 14 & 1139 & 11 & 1258 & 26 \\ 748 & 21 & 765 & 10 & 749 & 9 & 761 & 9 & 749 & 18 \\ 745 & 14 & 643 & 7 & 597 & 7 & 603 & 9 & 597 & 14 \\ 2285 & 9 & 2227 & 10 & 2109 & 21 & 2058 & 16 & 2285 & 18 \\ 1109 & 15 & 1175 & 11 & 1178 & 13 & 1285 & 16 & 1109 & 30 \\ 486 & 30 & 526 & 10 & 520 & 6 & 556 & 7 & 520 & 12 \\ 924 & 12 & 985 & 8 & 985 & 11 & 1118 & 12 & 985 & 22 \\ 1640 & 13 & 1664 & 13 & 1638 & 18 & 1770 & 17 & 1640 & 26 \\ 996 & 13 & 1028 & 9 & 1013 & 11 & 1086 & 10 & 996 & 26 \\ 497 & 30 & 573 & 10 & 576 & 7 & 596 & 6 & 497 & 60 \\ 1343 & 11 & 1366 & 10 & 1344 & 15 & 1515 & 14 & 1343 & 22 \\ 886 & 17 & 958 & 11 & 961 & 11 & 1037 & 10 & 961 & 22 \\ 1007 & 13 & 1027 & 9 & 1007 & 11 & 1189 & 13 & 1007 & 22 \\ 251 & 107 & 121 & 7 & 111 & 2 & 123 & 3 & 111 & 4 \\ 919 & 12 & 982 & 8 & 982 & 11 & 1695 & 14 & 982 & 22 \\ 929 & 53 & 831 & 21 & 772 & 11 & 884 & 16 & 772 & 22 \\ 636 & 15 & 697 & 8 & 695 & 8 & 778 & 9 & 695 & 16 \\ 728 & 30 & 634 & 11 & 590 & 7 & 765 & 13 & 590 & 14 \\ 1571 & 12 & 1620 & 12 & 1612 & 18 & 1780 & 19 & 1571 & 24 \\ 691 & 34 & 587 & 12 & 544 & 7 & 644 & 9 & 544 & 14 \\ 1551 & 11 & 1587 & 11 & 1568 & 17 & 1905 & 16 & 1551 & 22 \\ 782 & 13 & 788 & 7 & 767 & 9 & 715 & 6 & 767 & 18 \\ 821 & 56 & 784 & 12 & 771 & 9 & 769 & 9 & 771 & 18 \\ 1011 & 11 & 1081 & 8 & 1084 & 12 & 1138 & 10 & 1011 & 22 \\ & & & & & & & & & \end{array}$




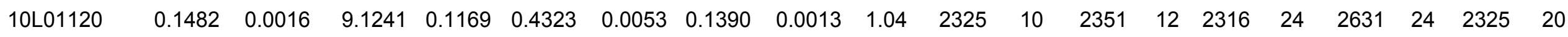

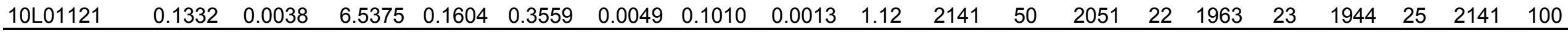

2010TQ01 (Qiuwu, Qiabulin section, $\mathrm{n}=105$, N29 $^{\circ} 20.180^{\prime} \quad \mathrm{E88}^{\circ} 30.687^{\prime}, 4075 \mathrm{~m}$ )

$\begin{array}{llllllllll}\text { 10TQ01-001 } & 0.0469 & 0.0243 & 0.0455 & 0.0236 & 0.0071 & 0.0003 & 0.0027 & 0.0002 & 0.60\end{array}$ $\begin{array}{llllllllll}\text { 10TQ01-002 } & 0.0473 & 0.0028 & 0.0222 & 0.0013 & 0.0034 & 0.0000 & 0.0011 & 0.0000 & 1.16\end{array}$

$\begin{array}{llllllllll}\text { 10TQ01-003 } & 0.0479 & 0.0078 & 0.0871 & 0.0140 & 0.0132 & 0.0003 & 0.0042 & 0.0002 & 0.90\end{array}$ 10TQ01-004 $0.0472 \quad 0.0029$ 10TQ01-005 $0.1562 \quad 0.0027$ 10TQ01-006 $0.0473 \quad 0.0033$ 10TQ01-007 $0.0496 \quad 0.0036$ 10TQ01-008 $0.0471 \quad 0.0256$ 10TQ01-009 $0.0764 \quad 0.0011$ 10TQ01-010 $0.0869 \quad 0.0024$ $\begin{array}{lll}10 T Q 01-011 & 0.0461 & 0.0049\end{array}$ 10TQ01-012 $0.0650 \quad 0.0013$ $\begin{array}{lll}10 T Q 01-013 & 0.0461 & 0.0036\end{array}$ $\begin{array}{lll}10 T Q 01-014 & 0.0609 & 0.0016\end{array}$ $\begin{array}{lll}10 T Q 01-015 & 0.0471 & 0.0127\end{array}$ $\begin{array}{lll}10 T Q 01-016 & 0.0479 & 0.0046\end{array}$ 10TQ01-017 $0.0497 \quad 0.0049$ 10TQ01-018 $0.0497 \quad 0.0153$ 10TQ01-019 $0.0484 \quad 0.0024$ $\begin{array}{lll}10 T Q 01-020 & 0.0483 & 0.0051\end{array}$ $\begin{array}{lll}10 T Q 01-021 & 0.0500 & 0.0023\end{array}$ $\begin{array}{lll}10 T Q 01-022 & 0.0602 & 0.0010\end{array}$ 10TQ01-023 $0.0484 \quad 0.0072$ $\begin{array}{lll}10 T Q 01-024 & 0.0615 & 0.0012\end{array}$ 10TQ01-025 $0.0505 \quad 0.0037$ 10TQ01-026 $0.0530 \quad 0.0020$ 10TQ01-027 $0.1075 \quad 0.0014$ 10TQ01-028 $0.0806 \quad 0.0013$ 10TQ01-029 $0.0484 \quad 0.0071$ 10TQ01-030 $0.0473 \quad 0.0135$ $\begin{array}{lllllll}0.0876 & 0.0053 & 0.0135 & 0.0002 & 0.0042 & 0.0001 & 1.62\end{array}$ $\begin{array}{lllllll}8.8680 & 0.1321 & 0.4118 & 0.0033 & 0.1150 & 0.0009 & 1.18\end{array}$ $\begin{array}{lllllll}0.0224 & 0.0015 & 0.0034 & 0.0000 & 0.0012 & 0.0000 & 0.93\end{array}$ $\begin{array}{llllllll}0.0973 & 0.0069 & 0.0142 & 0.0002 & 0.0051 & 0.0001 & 0.94\end{array}$ $\begin{array}{llllllll}0.0578 & 0.0311 & 0.0089 & 0.0006 & 0.0039 & 0.0005 & 0.83\end{array}$ $\begin{array}{llllllll}1.9501 & 0.0223 & 0.1851 & 0.0013 & 0.0488 & 0.0006 & 3.16\end{array}$ $\begin{array}{lllllll}2.7877 & 0.0722 & 0.2327 & 0.0026 & 0.0736 & 0.0012 & 1.01\end{array}$ $\begin{array}{lllllll}0.0569 & 0.0059 & 0.0090 & 0.0002 & 0.0031 & 0.0002 & 1.36\end{array}$ $\begin{array}{llllllll}1.0695 & 0.0196 & 0.1194 & 0.0009 & 0.0387 & 0.0005 & 1.53\end{array}$ $\begin{array}{llllllll}0.1073 & 0.0082 & 0.0169 & 0.0002 & 0.0055 & 0.0002 & 1.43\end{array}$ $\begin{array}{llllllll}0.8702 & 0.0218 & 0.1037 & 0.0009 & 0.0434 & 0.0010 & 4.31\end{array}$ $\begin{array}{llllllll}0.0600 & 0.0160 & 0.0092 & 0.0003 & 0.0025 & 0.0001 & 0.49\end{array}$ $\begin{array}{lllllll}0.0235 & 0.0022 & 0.0036 & 0.0001 & 0.0014 & 0.0001 & 1.30\end{array}$ $\begin{array}{lllllll}0.1720 & 0.0168 & 0.0251 & 0.0004 & 0.0073 & 0.0003 & 1.11\end{array}$ $\begin{array}{llllllll}0.1360 & 0.0418 & 0.0199 & 0.0006 & 0.0038 & 0.0005 & 0.91\end{array}$ $\begin{array}{lllllll}0.1312 & 0.0062 & 0.0197 & 0.0002 & 0.0043 & 0.0001 & 1.08\end{array}$ $\begin{array}{llllllll}0.0991 & 0.0103 & 0.0149 & 0.0003 & 0.0049 & 0.0002 & 0.78\end{array}$ $\begin{array}{lllllll}0.0993 & 0.0045 & 0.0144 & 0.0002 & 0.0046 & 0.0001 & 0.61\end{array}$ $\begin{array}{llllllll}0.7163 & 0.0100 & 0.0863 & 0.0006 & 0.0271 & 0.0002 & 1.17\end{array}$ $\begin{array}{lllllll}0.0558 & 0.0081 & 0.0084 & 0.0002 & 0.0026 & 0.0001 & 0.60\end{array}$ $\begin{array}{llllllll}0.6762 & 0.0119 & 0.0798 & 0.0006 & 0.0250 & 0.0003 & 2.27\end{array}$ $\begin{array}{lllllll}0.0649 & 0.0046 & 0.0093 & 0.0002 & 0.0029 & 0.0001 & 1.17\end{array}$ $\begin{array}{llllllll}0.2870 & 0.0105 & 0.0393 & 0.0004 & 0.0109 & 0.0002 & 1.20\end{array}$ $\begin{array}{lllllll}4.6869 & 0.0485 & 0.3162 & 0.0022 & 0.0902 & 0.0008 & 1.32\end{array}$ $\begin{array}{llllllll}2.2838 & 0.0317 & 0.2056 & 0.0015 & 0.0647 & 0.0007 & 1.48\end{array}$ $\begin{array}{llllllll}0.0516 & 0.0075 & 0.0077 & 0.0002 & 0.0026 & 0.0001 & 0.86\end{array}$ $\begin{array}{lllllll}0.0533 & 0.0151 & 0.0082 & 0.0003 & 0.0024 & 0.0002 & 0.66\end{array}$

$\begin{array}{cccccccccc}42 & 828 & 45 & 23 & 45 & 2 & 55 & 4 & 45 & 4 \\ 66 & 110 & 22 & 1 & 21.9 & 0.2 & 22.8 & 0.4 & 21.9 & 0.4 \\ 93 & 278 & 85 & 13 & 85 & 2 & 86 & 4 & 85 & 4 \\ 57 & 111 & 85 & 5 & 86 & 1 & 84 & 3 & 86 & 2 \\ 2415 & 29 & 2325 & 14 & 2223 & 15 & 2200 & 16 & 2415 & 58 \\ 63 & 127 & 22 & 2 & 22.1 & 0.3 & 23.6 & 0.6 & 22.1 & 0.6 \\ 178 & 130 & 94 & 6 & 91 & 1 & 103 & 3 & 91 & 2 \\ 56 & 824 & 57 & 30 & 57 & 4 & 78 & 10 & 57 & 8 \\ 1106 & 12 & 1098 & 8 & 1095 & 7 & 964 & 11 & 1106 & 24 \\ 1358 & 33 & 1352 & 19 & 1349 & 14 & 1435 & 23 & 1358 & 66 \\ & 214 & 56 & 6 & 58 & 1 & 62 & 5 & 58 & 2 \\ 773 & 25 & 739 & 10 & 727 & 5 & 768 & 9 & 727 & 10 \\ & 172 & 103 & 7 & 108 & 1 & 111 & 5 & 108 & 2 \\ 634 & 38 & 636 & 12 & 636 & 5 & 859 & 19 & 636 & 10 \\ 56 & 401 & 59 & 15 & 59 & 2 & 50 & 3 & 59 & 4 \\ 95 & 174 & 24 & 2 & 22.8 & 0.4 & 28 & 1 & 22.8 & 0.8 \\ 179 & 192 & 161 & 15 & 160 & 2 & 146 & 6 & 160 & 4 \\ 181 & 483 & 129 & 37 & 127 & 4 & 76 & 11 & 127 & 8 \\ 116 & 89 & 125 & 6 & 126 & 1 & 87 & 2 & 126 & 2 \\ 114 & 198 & 96 & 10 & 95 & 2 & 98 & 3 & 95 & 4 \\ 193 & 86 & 96 & 4 & 92.3 & 1 & 93 & 1 & 92.3 & 2 \\ 610 & 19 & 548 & 6 & 534 & 4 & 541 & 4 & 534 & 8 \\ 116 & 252 & 55 & 8 & 54 & 1 & 53 & 2 & 54 & 2 \\ 656 & 25 & 524 & 7 & 495 & 4 & 500 & 7 & 495 & 8 \\ 217 & 130 & 64 & 4 & 59.9 & 1 & 59 & 2 & 59.9 & 2 \\ 328 & 66 & 256 & 8 & 248 & 2 & 218 & 4 & 248 & 4 \\ 1758 & 9 & 1765 & 9 & 1771 & 11 & 1745 & 14 & 1758 & 18 \\ 1211 & 16 & 1207 & 10 & 1206 & 8 & 1267 & 13 & 1211 & 32 \\ 118 & 258 & 51 & 7 & 50 & 1 & 52 & 2 & 50 & 2 \\ 66 & 426 & 53 & 15 & 52 & 2 & 48 & 3 & 52 & 4\end{array}$




\begin{tabular}{|c|c|c|c|c|c|c|c|c|c|c|c|c|c|c|c|c|c|c|c|}
\hline 10TQ01-031 & .0495 & 0.0065 & 0.1011 & 0.0131 & 0.0148 & 0.0003 & 0.0045 & 0.0002 & 1.18 & 172 & 245 & 98 & 12 & 95 & 2 & 90 & 5 & 95 & 4 \\
\hline 10TQ01-032 & .0503 & 0.0056 & 0.1359 & 0.0149 & 0.0196 & 0.0003 & 0.0063 & 0.0002 & 0.85 & 210 & 219 & 129 & 13 & 125 & 2 & 128 & 4 & 125 & 4 \\
\hline OTQ01-033 & .0501 & 0.0071 & 0.1109 & 0.0155 & 0.0161 & 0.0004 & 0.0050 & 0.0002 & 0.84 & 198 & 262 & 107 & 14 & 103 & 0 & 101 & 5 & 103 & \\
\hline OTQ01-034 & .0605 & 0.0009 & 0.8622 & 0.0119 & 1034 & 0.0007 & 0.0319 & 0.0003 & 9.88 & 621 & 34 & 631 & 6 & 634 & 4 & 635 & 5 & 634 & \\
\hline 0TQ01-035 & 0.0482 & 0.0094 & 0.0511 & 0.0100 & .0077 & 0.0002 & 0.0020 & 0.0001 & 0.47 & 107 & 323 & 51 & 10 & 49 & 1 & 41 & 2 & 49 & \\
\hline OTQ01-036 & .0501 & 0.0057 & 0.0550 & 0.0061 & .0080 & 0.0002 & 0.0028 & 0.0001 & 0.75 & 198 & 211 & 54 & 6 & 51 & 1 & 56 & 2 & 51 & \\
\hline OTQ01-037 & 0.1156 & 0.0014 & 5.4285 & .0509 & 3406 & 0.0023 & 0.0773 & 0.0008 & 2.05 & 1890 & 8 & 1889 & ర & 1889 & 11 & 1505 & 15 & 1890 & 6 \\
\hline DTQ01 & 0.2611 & 0.0033 & 1.4230 & 0.2178 & 0.5952 & 0.0050 & 0.1318 & 0.0014 & 0.97 & 3253 & 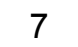 & 158 & 10 & 3011 & 0 & 2502 & 25 & 3253 & 4 \\
\hline OTQ01 & 0.0509 & .0063 & 0.1260 & 0153 & 30 & .0005 & 0.0056 & 0004 & 1.57 & 23 & 225 & 120 & 14 & 115 & 0 & 113 & & 115 & 6 \\
\hline 40 & .0758 & .0012 & 1.8936 & .0257 & 3 & .0013 & 0.0523 & .0004 & 0.97 & 090 & 16 & 1079 & 9 & 1074 & & 031 & & 1090 & 32 \\
\hline 41 & 0798 & .0011 & 1.7829 & .0190 & 1620 & .0011 & 0.0329 & 0003 & 1.60 & 193 & 11 & 1039 & $r$ & 968 & & 653 & & 968 & 2 \\
\hline 42 & 0485 & 0117 & 0.0919 & .0221 & .0138 & 0004 & 0.0046 & 03 & 1.00 & 122 & 380 & 89 & 21 & 88 & & 92 & & 88 & \\
\hline OTQ01-043 & 0479 & 0.0063 & 0.0586 & 0.0077 & 0.0089 & .0002 & 0.0032 & 0.0001 & 0.99 & 96 & 245 & 58 & 7 & 56.9 & & 64 & 2 & 56.9 & \\
\hline OTQ01-044 & .0903 & 0.0012 & 3.1001 & 0.0330 & 0.2492 & .0017 & 0.0744 & 0.0006 & 1.11 & 1431 & 10 & 1433 & 8 & 1434 & $s$ & 1451 & 11 & 1431 & 20 \\
\hline 045 & 0597 & 0.0011 & 0.8119 & 0.0125 & 0.0986 & .0007 & 0.0302 & .0003 & 1.21 & 593 & 21 & 604 & 7 & 606 & 4 & 602 & & 606 & 8 \\
\hline 10TQ01-046 & .0495 & 0.0038 & 0.1333 & 0.0099 & 0.0195 & .0003 & 0.0060 & 0.0004 & 3.99 & $1 \%$ & 138 & 121 & 9 & 125 & 2 & 121 & 8 & 125 & 7 \\
\hline 10TQ01-047 & .0624 & 0.0019 & 0.7841 & 0.0223 & 0.0912 & 0.0009 & 0.0262 & 0.0005 & 1.71 & 688 & 44 & 588 & 13 & 562 & 5 & 523 & 10 & 562 & 0 \\
\hline 10TQ01-048 & .0755 & 0.0013 & 1.6576 & 0.0256 & 0.1593 & 0.0012 & 0.0425 & 0.0005 & 1.40 & 1082 & 19 & 992 & 10 & 953 & 7 & 842 & 9 & 953 & 14 \\
\hline 10TQ & 0584 & 0.0012 & 0.6920 & 0.0130 & .0859 & 0.0007 & 0.0264 & 0.0002 & 0.56 & 54 & 28 & 534 & 8 & 531 & 4 & 527 & 4 & 531 & \\
\hline 10TQ & 0.0489 & 0.0022 & 0.0512 & 0.0022 & 0.0076 & 0.0001 & 0.0026 & 0.0001 & 1.19 & 143 & 80 & 51 & 2 & 48.7 & 0.5 & 52 & 1 & 48.7 & 1 \\
\hline 10TQ01-051 & 0.0723 & 0.0013 & 1.4034 & 0.0222 & 0.1409 & 0.0011 & 0.0336 & 0.0036 & 32.23 & 994 & 20 & 890 & 9 & 849 & 6 & 668 & 69 & 849 & 12 \\
\hline 10TQ01-052 & 0.0500 & 0.0052 & 0.1103 & 0.0113 & 0.0160 & 0.0003 & 0.0049 & 0.0002 & 0.85 & 194 & 193 & 106 & 10 & 102 & 2 & 99 & 3 & 102 & 4 \\
\hline 10TQ01-053 & 0.1238 & 0.0021 & 6.0291 & 0.0883 & 0.3534 & 0.0031 & 0.0864 & 0.0009 & 0.62 & 2011 & 14 & 1980 & 13 & 1951 & 15 & 1676 & 16 & 2011 & 28 \\
\hline 10TC & 0.0862 & 0.0027 & 2.7575 & 0.0812 & 0.2320 & 0.0024 & 0.0643 & 0.0009 & 0.56 & 1343 & 41 & 1344 & 22 & 1345 & 13 & 1259 & 16 & 1343 & 82 \\
\hline 10TQ & 0.0492 & 0.0052 & & 0.0168 & .0238 & 0.0003 & & 0.0003 & 0.96 & 155 & 210 & 152 & 15 & 152 & 0 & 147 & 5 & 152 & 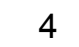 \\
\hline 10TQ01-( & 0.0496 & 0.0036 & & 0.0064 & 0.0 & 0.0002 & & 0.0001 & 1.28 & 176 & 139 & 86 & 6 & 83 & 1 & 83 & 2 & 83 & 2 \\
\hline 10TQ01-057 & 0.1094 & 0.0013 & & 0.0410 & 09 & 0.0021 & & 11 & 5.03 & 1790 & 7 & 1792 & 7 & 1794 & 10 & 1922 & 20 & 1790 & 4 \\
\hline 10TC & 0.0775 & 0.0009 & & 0.0 & & 0.0012 & & 05 & 3.82 & 1134 & 9 & 1110 & 6 & 1098 & 6 & 992 & 9 & 1134 & 8 \\
\hline $10 \mathrm{TC}$ & 0484 & 0.0069 & 19 & .0116 & 0123 & 0.0002 & 0.0039 & & 0.6 & 11 & 261 & 80 & 11 & 79 & 1 & 78 & 2 & 79 & 2 \\
\hline 10TQ01-060 & .0707 & 0.0012 & 1.5572 & .0217 & 1599 & 0.0012 & 0.0496 & 0.0006 & $3 .($ & 94 & 17 & 953 & 9 & 956 & 6 & 978 & 12 & 956 & ? \\
\hline 10TC & 0488 & 0.0021 & 0.0816 & 0034 & 0121 & .0001 & 0.0037 & 0.0000 & 0. & 14 & 79 & 80 & 3 & 77.7 & 0.7 & 74.8 & 0.8 & 77.7 & \\
\hline & & .0013 & & & .2286 & 017 & & 008 & 1.79 & 1353 & 14 & 1337 & 10 & 1327 & 9 & 1435 & 14 & 1353 & 28 \\
\hline OTC & 0.0503 & 0.0032 & 0.0226 & 0014 & 0.0033 & 0.0001 & 0.0011 & 0.0000 & 1.79 & 207 & 114 & 23 & 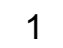 & 21 & 0.3 & 22.6 & 0.8 & 21 & .6 \\
\hline 10TQ01-064 & 0.0716 & 0.0010 & 1.5889 & 0.0178 & 0.1610 & .0011 & 0.0482 & 0.0004 & 1.86 & 975 & 12 & 966 & 7 & 962 & 6 & 951 & 8 & 962 & 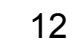 \\
\hline
\end{tabular}




\begin{tabular}{|c|c|c|c|c|c|c|c|c|c|c|c|c|c|c|c|c|c|c|c|}
\hline 10TQ01-065 & .0485 & 0.0069 & 0.1060 & 0.0148 & 0.0159 & 0.0004 & 0.0055 & 0.0002 & 0.91 & 122 & 255 & 102 & 14 & 102 & 2 & 111 & 5 & 102 & 4 \\
\hline OTQ01-066 & 0498 & 0.0075 & 0.0546 & 0.0082 & 0.0080 & 0.0001 & 0.0027 & 0.0001 & 0.55 & 184 & 280 & 54 & 8 & 51.1 & 0.8 & 55 & 1 & 1.1 & .6 \\
\hline OTQ01-067 & .0750 & 0.0010 & 1.8274 & 0.0201 & 0.1768 & 0.0012 & 0.0520 & 0.0004 & 1.20 & 1068 & 12 & 1055 & 7 & 1049 & 7 & 1024 & 7 & 1068 & 24 \\
\hline 0TQ01-068 & .0724 & 0.0012 & 1.4765 & 0.0221 & 0.1480 & 0.0011 & 0.0448 & 0.0006 & 2.54 & 997 & 18 & 921 & 9 & 890 & 6 & 885 & 11 & 890 & 12 \\
\hline OTQ01-069 & .0487 & 0.0094 & 0.0841 & 0.0161 & .0125 & 0.0003 & 0.0039 & 0.0001 & 0.40 & 132 & 321 & 82 & 15 & 80 & 2 & 78 & 2 & 80 & 4 \\
\hline OTQ01-070 & .0486 & 0.0023 & 0.1224 & 0.0056 & .0183 & 0.0002 & 0.0055 & 0.0001 & 0.63 & 126 & 88 & 117 & 5 & 117 & 1 & 111 & 1 & 117 & 2 \\
\hline DTQ01-071 & 0.0689 & 0.0015 & 1.3345 & .0270 & 1404 & 0.0012 & 0.0362 & 0.0013 & 6.37 & 897 & 28 & 861 & 12 & 847 & 7 & 719 & 25 & 847 & 14 \\
\hline DTQ01 & 0.0475 & 0.0059 & 0.0211 & 0.0026 & 0.0032 & 0.0001 & 0.0010 & 0000 & 0.93 & 73 & 228 & 21 & 3 & 20.7 & 0.3 & 20.6 & 0.8 & 20.7 & 0.6 \\
\hline DTQ01-073 & 0.06 & 0046 & 0.8932 & 3 & 0.0987 & 0.0020 & 0.0165 & 10 & 1.70 & 795 & 110 & 648 & 33 & 607 & 12 & 30 & 20 & 7 & 24 \\
\hline 74 & .0494 & .0058 & 0.0514 & 0060 & .0076 & .0001 & 0.0024 & 0001 & 0.54 & 5 & 231 & 51 & & 48.6 & 0.8 & 49 & 1 & 3.6 & 6 \\
\hline 75 & 1596 & 0019 & 9.9251 & .0857 & 4512 & 0031 & 0.1037 & 0012 & 2.91 & 451 & 7 & 2428 & & 2400 & 14 & 1995 & 21 & 2451 & 14 \\
\hline 576 & 0594 & 0010 & 0.7458 & ro10/ & 0911 & 0006 & 0.0265 & 02 & 0.78 & 581 & 19 & 566 & & 562 & & 529 & 4 & 562 & 8 \\
\hline 0TQ01-077 & 0835 & 0027 & 2.5063 & 0.0785 & 2178 & .0024 & 0.0579 & 0012 & 1.00 & 1280 & 4 & 1274 & 23 & 1270 & 13 & 1137 & 23 & 1280 & 88 \\
\hline OTQ01-078 & .0480 & 0.0212 & 0.1151 & 0.0508 & 0.0174 & .0005 & 0.0064 & 0.0006 & 0.94 & 97 & 699 & 111 & 46 & 111 & & 128 & 3 & 111 & 6 \\
\hline 079 & 0675 & 0062 & 1.1173 & 1011 & 1200 & .0029 & 0.0293 & 0008 & 0.34 & 85 & 149 & 762 & 48 & 731 & 16 & 584 & 15 & 731 & 32 \\
\hline 10TQ01-080 & .0502 & 0.0021 & 0.1642 & 0.0067 & 0.0237 & .0003 & 0.0079 & 0.0001 & 0.90 & 203 & 74 & 154 & 6 & 151 & 0 & 159 & 3 & 151 & 4 \\
\hline 10TQ01-081 & 0527 & 0.0049 & 0.1092 & 0.0100 & 0.0150 & 0.0002 & 0.0047 & 0.0001 & 0.90 & 314 & 212 & 105 & 9 & 96 & 1 & 95.1 & 1 & 96 & 2 \\
\hline 10TQ01-082 & 1456 & 0.0016 & 8.5777 & 0.0675 & 0.4275 & 0.0028 & 0.1146 & 0.0009 & 1.76 & 2294 & 6 & 2294 & 7 & 2294 & 13 & 2193 & 16 & 294 & 12 \\
\hline OTQ01-083 & .2226 & 0.0024 & 17.5832 & 0.1335 & 0.5730 & 0.0039 & 0.1512 & 0.0013 & 2.03 & 3000 & & 2967 & 7 & 2920 & 16 & 2846 & 22 & 3000 & 10 \\
\hline 10TC & 0822 & 0.0010 & 2.3919 & .0224 & 2111 & 0.0014 & 0.0560 & 0.0004 & 1.15 & 1250 & 9 & 1240 & 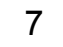 & 1235 & 7 & 1102 & 7 & 1250 & 18 \\
\hline 10TQ01-085 & 0.0728 & 0.0011 & 1.6763 & 0.0198 & 0.1671 & 0.0011 & 0.0499 & 0.0004 & 0.93 & 1008 & 13 & 1000 & 8 & 996 & 6 & 984 & 7 & 996 & 12 \\
\hline 10TQ & 0.0489 & 0.0044 & 0.0587 & 0.0051 & .0087 & 0.0002 & 0.0028 & 0.0001 & 0.94 & 142 & 161 & 58 & 5 & 56 & 1 & 56 & 2 & 56 & 2 \\
\hline 10TQ01-087 & 0.0819 & 0.0012 & 2.4251 & 0.0308 & 0.2149 & 0.0016 & 0.0609 & 0.0005 & 0.84 & 1242 & 14 & 1250 & 9 & 1255 & 0 & 1196 & 9 & 1242 & 28 \\
\hline 10TC & 0.0522 & 0.0050 & 0.1274 & 0.0119 & .0177 & 0.0004 & 0.0065 & 0.0002 & 0.80 & 293 & 173 & 122 & 11 & 113 & 2 & 130 & 4 & 113 & 4 \\
\hline 10TC & 0.0909 & 0.0015 & 2.9914 & 0.0429 & 0.2 & 0.0018 & & & 1.79 & 1444 & 16 & 1405 & 11 & 1380 & 10 & 1137 & 15 & 1444 & 32 \\
\hline 10TQ01- & 0.1 & 0.0021 & & 0.07 & 2722 & 0.0024 & & 12 & 1.60 & 1695 & 21 & 1613 & 15 & 1552 & 12 & 682 & 24 & 1695 & 42 \\
\hline $10 \mathrm{TC}$ & 0.0 & 0.0078 & & 0.0 & 0222 & 0.0007 & & & 0.64 & 20 & 273 & 145 & 20 & 141 & 1 & 137 & 6 & 141 & 8 \\
\hline $10 \mathrm{TC}$ & 0. & 0.0080 & & & 38 & 0.0 & & 02 & 0.99 & 2 & 278 & 62 & 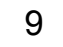 & 56 & 2 & 63 & 3 & 56 & 4 \\
\hline $10 \mathrm{TC}$ & 86 & 0.0026 & 1084 & .0055 & .0162 & 0.0002 & 0.0048 & & 1.47 & 10 & 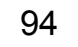 & 105 & 5 & 10 & 1 & 91 & 2 & 103 & 2 \\
\hline 10TQ01-094 & 0506 & 0.0042 & .0665 & .0054 & .0095 & 0.0002 & 0.0030 & 0.0001 & 0.66 & 22 & 15 & 65 & 5 & 61 & 1 & 61 & 2 & 61 & 2 \\
\hline 10TC & 0485 & .0060 & .0486 & .0060 & .0073 & .0001 & 0.0021 & 0.0001 & 0.5 & 12 & 240 & 48 & 6 & 46.7 & 0.7 & 42 & 1 & 46.7 & 1.4 \\
\hline & & 0033 & & & & 003 & & & 0.77 & 162 & 11 & 128 & 0 & 127 & 2 & 120 & 3 & 127 & 4 \\
\hline OT & & 0.0093 & .0962 & 0185 & 0.0146 & 0.0003 & & 0.0003 & 0.95 & y & 314 & 93 & 17 & 93 & 2 & 107 & 5 & 93 & 4 \\
\hline 10TQ01-098 & 0.0493 & 0.0035 & 0.0785 & 0.0054 & 0.0116 & 0.0002 & 0.0036 & 0.0001 & 0.76 & 164 & 131 & 77 & 5 & 74 & 1 & 72 & 2 & 74 & 2 \\
\hline
\end{tabular}




\begin{tabular}{|c|c|c|c|c|c|c|c|c|c|c|c|c|c|c|c|c|c|c|c|}
\hline 10TQ01-099 & 0.0475 & 0.0113 & 0.0521 & 0.0123 & 0.0080 & 0.0002 & 0.0029 & 0.0001 & 0.57 & 73 & 368 & 52 & 12 & 51 & 1 & 58 & 2 & 51 & 2 \\
\hline 10TQ01-100 & 0.0487 & 0.0027 & 0.1111 & 0.0061 & 0.0166 & 0.0002 & 0.0050 & 0.0001 & 1.08 & 131 & 99 & 107 & 6 & 106 & 1 & 101 & 2 & 106 & 2 \\
\hline 10TQ01-101 & 0.0575 & 0.0056 & 0.4648 & 0.0445 & 0.0587 & 0.0011 & 0.0179 & 0.0008 & 1.19 & 509 & 180 & 388 & 31 & 368 & 7 & 359 & 16 & 368 & 14 \\
\hline 10TQ01-102 & 0.0597 & 0.0021 & 0.7841 & 0.0269 & 0.0952 & 0.0010 & 0.0288 & 0.0004 & 0.81 & 593 & 57 & 588 & 15 & 586 & 6 & 574 & 8 & 586 & 12 \\
\hline 10TQ01-103 & 0.0472 & 0.0049 & 0.0880 & 0.0091 & 0.0135 & 0.0002 & 0.0041 & 0.0001 & 0.98 & 61 & 204 & 86 & 8 & 87 & 1 & 83 & 3 & 87 & \\
\hline I0TQ01-104 & 0.0588 & 0.0016 & 0.7003 & 0.0174 & 0.0865 & 0.0007 & 0.0210 & 0.0008 & 5.24 & 558 & 40 & 539 & 10 & 535 & 4 & 420 & 16 & 535 & 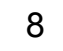 \\
\hline I0TQ01-105 & 0.0504 & 0.0011 & 0.2407 & 0.0047 & 0.0347 & 0.0003 & 0.0106 & 0.0001 & 0.88 & 213 & 31 & 219 & 4 & 220 & 2 & 213 & 2 & 220 & 4 \\
\hline
\end{tabular}

2011TQ03 (Qiuwu, Qiabulin section, $\mathrm{n}=80, \mathrm{~N}^{\circ} 9^{\circ} 20.126^{\prime} \quad \mathrm{E}^{\circ} 8^{\circ} 30.426^{\prime}, 4054 \mathrm{~m}$ )

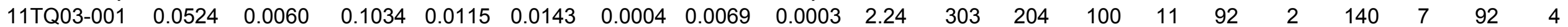

$\begin{array}{lllllllllllllllllllllll}\text { 11TQ03-002 } & 0.0509 & 0.0032 & 0.0418 & 0.0025 & 0.0060 & 0.0001 & 0.0036 & 0.0002 & 12.24 & 235 & 112 & 42 & 2 & 38.3 & 0.6 & 72 & 4 & 38.3 & 1.2\end{array}$

$\begin{array}{llllllllllllllllllll}\text { 11TQ03-003 } & 0.0798 & 0.0012 & 2.0699 & 0.0269 & 0.1881 & 0.0015 & 0.0528 & 0.0005 & 1.34 & 1193 & 14 & 1139 & 9 & 1111 & 8 & 1039 & 9 & 1193 & 28\end{array}$

$\begin{array}{llllllllllllllllllll}\text { 11TQ03-004 } & 0.0627 & 0.0032 & 0.7546 & 0.0370 & 0.0874 & 0.0013 & 0.0272 & 0.0005 & 0.65 & 696 & 79 & 571 & 21 & 540 & 8 & 543 & 9 & 540 & 16\end{array}$

$\begin{array}{llllllllllllllllllllll}\text { 11TQ03-005 } & 0.0527 & 0.0012 & 0.2349 & 0.0048 & 0.0323 & 0.0003 & 0.0102 & 0.0001 & 4.42 & 314 & 51 & 214 & 4 & 205 & 2 & 204 & 2 & 205 & 4\end{array}$

$\begin{array}{lllllllllllllllllllll}\text { 11TQ03-006 } & 0.0536 & 0.0050 & 0.1571 & 0.0142 & 0.0212 & 0.0005 & 0.0065 & 0.0002 & 0.86 & 356 & 163 & 148 & 12 & 135 & 3 & 131 & 4 & 135 & 6\end{array}$

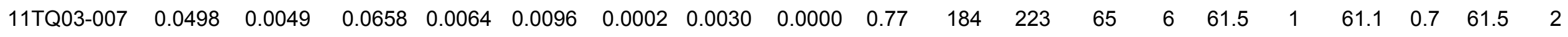

$\begin{array}{lllllllllllllllllll}\text { 11TQ03-008 } & 0.0461 & 0.0028 & 0.0510 & 0.0030 & 0.0080 & 0.0001 & 0.0026 & 0.0001 & 1.29 & 130 & 50 & 3 & 51.5 & 0.7 & 52 & 1 & 51.5 & 1.4\end{array}$

$\begin{array}{lllllllllllllllllllll}\text { 11TQ03-009 } & 0.0527 & 0.0016 & 0.1040 & 0.0031 & 0.0143 & 0.0001 & 0.0051 & 0.0001 & 1.50 & 318 & 49 & 100 & 3 & 91.5 & 0.9 & 102 & 1 & 91.5 & 1.8\end{array}$

$\begin{array}{llllllllllllllllllll}\text { 11TQ03-010 } & 0.0674 & 0.0009 & 1.2158 & 0.0131 & 0.1309 & 0.0010 & 0.0399 & 0.0004 & 17.54 & 849 & 28 & 808 & 6 & 793 & 6 & 790 & 7 & 793 & 12\end{array}$

$\begin{array}{llllllllllllllllllllll}\text { 11TQ03-011 } & 0.0504 & 0.0057 & 0.0492 & 0.0055 & 0.0071 & 0.0002 & 0.0028 & 0.0001 & 1.33 & 214 & 200 & 49 & 5 & 45 & 1 & 56 & 2 & 45 & 2\end{array}$

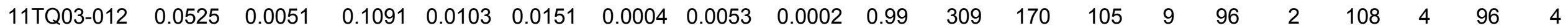

$\begin{array}{lllllllllllllllllllll}\text { 11TQ03-013 } & 0.0521 & 0.0046 & 0.1058 & 0.0092 & 0.0147 & 0.0003 & 0.0050 & 0.0002 & 1.12 & 290 & 161 & 102 & 8 & 94 & 2 & 101 & 3 & 94 & 4\end{array}$

$\begin{array}{llllllllllllllllllll}\text { 11TQ03-014 } & 0.0739 & 0.0024 & 1.6652 & 0.0520 & 0.1635 & 0.0020 & 0.0514 & 0.0011 & 1.96 & 1039 & 43 & 995 & 20 & 976 & 11 & 1013 & 21 & 976 & 22\end{array}$

$\begin{array}{llllllllllllllllllllll}11 \text { TQ03-015 } & 0.0511 & 0.0036 & 0.0567 & 0.0038 & 0.0081 & 0.0001 & 0.0031 & 0.0001 & 1.23 & 247 & 124 & 56 & 4 & 51.7 & 0.8 & 62 & 2 & 51.7 & 1.6\end{array}$

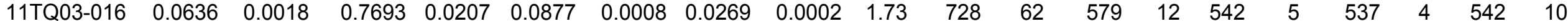

$\begin{array}{llllllllllllllllllll}\text { 11TQ03-017 } & 0.1138 & 0.0013 & 4.7844 & 0.0421 & 0.3051 & 0.0023 & 0.0771 & 0.0007 & 3.39 & 1860 & 7 & 1782 & 7 & 1716 & 11 & 1501 & 13 & 1860 & 14\end{array}$

$\begin{array}{lllllllllllllllllllll}\text { 11TQ03-018 } & 0.0484 & 0.0067 & 0.0560 & 0.0076 & 0.0084 & 0.0003 & 0.0026 & 0.0001 & 1.09 & 119 & 239 & 55 & 7 & 54 & 2 & 52 & 3 & 54 & 4\end{array}$

$\begin{array}{lllllllllllllllllllll}\text { 11TQ03-019 } & 0.0512 & 0.0076 & 0.0644 & 0.0093 & 0.0091 & 0.0003 & 0.0025 & 0.0001 & 0.76 & 251 & 260 & 63 & 9 & 59 & 2 & 50 & 3 & 59 & 4\end{array}$

$\begin{array}{llllllllllllllllllll}\text { 11TQ03-020 } & 0.0509 & 0.0053 & 0.0625 & 0.0065 & 0.0089 & 0.0001 & 0.0028 & 0.0001 & 1.42 & 234 & 239 & 62 & 6 & 57.2 & 0.8 & 57 & 1 & 57.2 & 1.6\end{array}$

$\begin{array}{lllllllllllllllllllll}\text { 11TQ03-021 } & 0.0612 & 0.0019 & 0.7480 & 0.0222 & 0.0886 & 0.0010 & 0.0272 & 0.0005 & 1.82 & 647 & 45 & 567 & 13 & 547 & 6 & 542 & 10 & 547 & 12\end{array}$

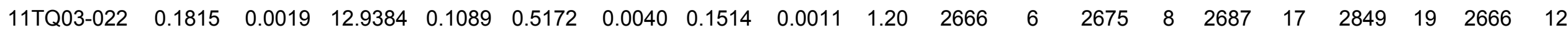

$\begin{array}{lllllllllllllllllllll}\text { 11TQ03-023 } & 0.0709 & 0.0009 & 1.5529 & 0.0168 & 0.1589 & 0.0012 & 0.0501 & 0.0004 & 1.77 & 955 & 11 & 952 & 7 & 950 & 7 & 988 & 7 & 950 & 14\end{array}$

$\begin{array}{lllllllllllllllllll}\text { 11TQ03-024 } & 0.0461 & 0.0046 & 0.0500 & 0.0049 & 0.0079 & 0.0002 & 0.0026 & 0.0001 & 1.25 & 210 & 50 & 5 & 51 & 1 & 52 & 2 & 51 & 2\end{array}$

$\begin{array}{llllllllllllllllllll}11 \mathrm{TQ} 03-025 & 0.0483 & 0.0038 & 0.0542 & 0.0042 & 0.0081 & 0.0002 & 0.0024 & 0.0001 & 0.48 & 114 & 138 & 54 & 4 & 52.3 & 1 & 49 & 1 & 52.3 & 2\end{array}$ 


\begin{tabular}{|c|c|c|c|c|c|c|c|c|c|c|c|c|c|c|c|c|c|c|c|}
\hline 1TQ03-026 & 0.0594 & 0.0013 & 0.7082 & 0.0142 & 0.0865 & 0.0008 & 0.0261 & 0.0002 & 0.70 & 581 & 28 & 544 & 8 & 535 & 5 & 521 & 5 & 535 & 10 \\
\hline 11TQ03-027 & 0.0704 & 0.0016 & 1.4519 & 0.0317 & 0.1497 & 0.0015 & 0.0462 & 0.0005 & 0.90 & 939 & 29 & 911 & 13 & 899 & 8 & 913 & 10 & 899 & 16 \\
\hline 1TQ03-028 & 0.0480 & 0.0038 & 0.0659 & 0.0051 & 0.0100 & 0.0002 & 0.0031 & 0.0001 & 0.50 & 99 & 138 & 65 & 5 & 64 & 1 & 63 & 1 & 64 & 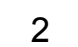 \\
\hline 1TQ03-029 & 0.0489 & 0.0110 & 0.0623 & 0.0137 & 0.0092 & 0.0005 & 0.0033 & 0.0002 & 0.79 & 144 & 8 & 61 & 13 & 59 & 3 & 67 & 4 & 59 & \\
\hline 1TQ03-030 & 0.0486 & 0.0051 & 0.0619 & 0.0063 & 0.0092 & 0.0002 & 0.0029 & 0.0001 & 1.51 & 127 & 232 & 61 & 6 & 59 & 4 & 59 & 2 & 59 & \\
\hline 1TQ03-031 & 0.0490 & 0.0071 & 0.1106 & 0.0156 & 0.0164 & 0.0005 & 0.0067 & 0.0004 & 1.28 & 150 & 251 & 106 & 14 & 105 & 0 & 135 & 7 & 105 & \\
\hline TQ03-032 & 0.0516 & 0102 & 0.0586 & 0.0114 & 0.0082 & 0.0004 & 0.0033 & 0.0002 & 0.79 & 268 & 323 & 58 & 11 & 53 & 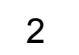 & 67 & 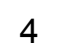 & 53 & \\
\hline TQ03-033 & 0.0492 & 0064 & 0.0920 & 0.0118 & 0.0136 & .0003 & 0.0043 & 0.0002 & .30 & 155 & 269 & 89 & 11 & 87 & & 87 & 3 & 87 & \\
\hline ITQ03-034 & 0.2129 & 0030 & 16.6922 & 0.1883 & 5687 & 0049 & 0.1542 & 13 & 1.73 & 2928 & 24 & 917 & 11 & 2902 & 20 & 2898 & 23 & 928 & \\
\hline TQ03-035 & 0.0515 & 0099 & 0.0615 & 0.0115 & 0.0087 & 0004 & 0.0032 & 002 & 0.93 & 261 & 310 & 61 & 11 & 56 & . & 64 & & 56 & \\
\hline ITQ03-036 & 0.0721 & .0022 & 1.6290 & 0.0487 & 0.1639 & 0.0019 & 0.0541 & 0.0010 & 1.39 & 989 & 41 & 981 & 19 & 978 & 11 & 064 & 18 & 978 & \\
\hline 1TQ03-037 & 0.0770 & 0.0010 & 2.0161 & 0.0230 & 0.1899 & 0.0015 & 0.0578 & 0.0005 & 1.65 & 1122 & 11 & 1121 & 0 & 1121 & 0 & 1136 & 9 & 122 & \\
\hline 1TQ03-038 & 0.0515 & 0.0017 & 0.0449 & 0.0014 & 0.0063 & 0.0001 & 0.0025 & 0.0001 & 3.41 & 264 & 56 & 45 & 1 & 40.6 & 0.4 & 50 & 1 & 40.6 & \\
\hline 11TQ03-039 & 0.0819 & 0.0016 & 2.4036 & 0.0424 & 0.2128 & 0.0020 & 0.0652 & 0.0008 & 1.53 & 1244 & 20 & 1244 & 13 & 1244 & 11 & 1277 & 15 & 1244 & \\
\hline 1TQ03-040 & 0.0623 & 0.0013 & 0.7432 & 0.0142 & 0.0865 & 0.0008 & 0.0281 & 0.0002 & 0.54 & 685 & 26 & 564 & 8 & 535 & 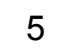 & 560 & 4 & 535 & \\
\hline 1TQ03-041 & 0.0517 & 0.0045 & 0.1027 & 0.0086 & 0.0144 & 0.0003 & 0.0051 & 0.0002 & 1.28 & 270 & 152 & 99 & 8 & 92 & 2 & 102 & 4 & 92 & \\
\hline 3-042 & 0.0811 & 0.0009 & 2.3718 & 0.0224 & 0.2121 & 0.0016 & 0.0644 & 0.0004 & 0.74 & 1225 & 8 & 1234 & 7 & 1240 & 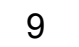 & 1262 & 8 & 1225 & \\
\hline ITQ03-043 & 0.0503 & 0.0113 & 0.0708 & 0.0156 & 0.0102 & 0.0005 & 0.0036 & 0.0003 & 1.31 & 211 & 339 & 69 & 15 & 65 & 3 & 72 & 6 & 65 & \\
\hline 1TQ03-044 & 0.1014 & 0.0012 & 4.0544 & 0.0410 & 0.2901 & 0.0023 & 0.0859 & 0.0008 & 2.22 & 1650 & 9 & 1645 & 8 & 1642 & 11 & 1666 & 14 & 1650 & 0 \\
\hline 1TQ03-045 & 0.0787 & 0.0014 & 2.1471 & 0.0342 & 0.1979 & 0.0018 & 0.0627 & 0.0009 & 3.37 & 1165 & 18 & 1164 & 11 & 1164 & 9 & 1229 & 18 & 1165 & 0 \\
\hline 1TQ03-046 & 0.0768 & 0.0015 & 1.8825 & 0.0340 & 0.1777 & 0.0016 & 0.0597 & 0.0007 & 1.53 & 1117 & 22 & 1075 & 12 & 1055 & 9 & 1173 & 13 & 1117 & 4 \\
\hline 1TQ03-047 & 0.0496 & 0.0175 & 0.0580 & 0.0201 & 85 & 0.0006 & 0.0 & 03 & 0.63 & 176 & 487 & 57 & 19 & 54 & 4 & 56 & 5 & 54 & \\
\hline 1TQ03-048 & 0.0527 & 0.0039 & 0.1679 & 0.0120 & 0.0231 & 0.0004 & 0.0089 & 03 & 1.38 & 315 & 129 & 158 & 10 & 147 & 3 & 179 & 5 & 47 & \\
\hline 1TQ03-049 & 0.0524 & 0.0110 & 0.1 & 0.0232 & 0.0156 & & & 05 & 1.64 & 304 & 340 & 109 & 21 & 100 & 4 & 115 & 10 & 00 & \\
\hline $1 \mathrm{TC}$ & 0.0 & 0.0 & & 0.0100 & 0.0 & & & 01 & 1.49 & 127 & 183 & 11 & 9 & 119 & 2 & 9 & 2 & 19 & \\
\hline $11 \mathrm{TC}$ & 0.0 & 0084 & 0.1 & 0.0183 & 2 & 0.0006 & 0.0055 & 03 & 0.73 & 291 & 282 & 11 & 17 & 10 & 4 & 11 & 6 & 4 & \\
\hline 52 & 32 & 0036 & 30 & 0.0080 & .0167 & 0.0003 & 0.0078 & 04 & 5.2 & 157 & 127 & 10 & 7 & 107 & 2 & 15 & 8 & 107 & \\
\hline 1TQ03-053 & 0.0528 & 0.0046 & 0.1028 & 0.0086 & 0.0141 & 0.0003 & 0.0051 & 0.0002 & 1.09 & 319 & 152 & 99 & 8 & 90 & 2 & 103 & 3 & 90 & \\
\hline 1TQ03-054 & 0.0492 & 0.0034 & 0.1075 & 0.0073 & 0.0159 & 0.0002 & 0.0050 & 0.0001 & 1.46 & 157 & 156 & 10 & 7 & 101 & 1 & 101 & 1 & 101 & \\
\hline 11TQ03-055 & 0.2255 & 0.0022 & 18.4715 & 0.1376 & 0.5942 & 0.0045 & 0.1679 & 0.0012 & 2.81 & 3021 & 6 & 3015 & 7 & 3006 & 18 & 3138 & 20 & 3021 & 2 \\
\hline 11TQ03-056 & 0.0473 & .0016 & 0.0476 & 0.0016 & 0.0073 & 0.0001 & 0.0023 & 0.0000 & 0.94 & 62 & 57 & 47 & 2 & 46.9 & 0.4 & 46 & 0.6 & 46.9 & \\
\hline 11TQ03-057 & 0.0497 & 0.0041 & 0.0732 & 0.0060 & 0.0107 & 0.0002 & 0.0038 & 0.0001 & 1.32 & 180 & 148 & 72 & 6 & 69 & 1 & 76 & 3 & 69 & \\
\hline 1TQ03-058 & 0.0762 & .0009 & 1.8853 & 0.0186 & 0.1794 & .0014 & 0.0572 & 0.0004 & 1.56 & 1101 & 9 & 1076 & 7 & 1064 & 8 & 1123 & 8 & 1101 & \\
\hline 1TQ03-059 & 0.1137 & 0.0015 & 5.1925 & 0.0572 & 0.3314 & 0.0028 & 0.1007 & 0.0009 & 1.25 & 1859 & 9 & 1851 & 9 & 1845 & 13 & 1940 & 16 & 1859 & \\
\hline
\end{tabular}




\begin{tabular}{|c|c|c|c|c|c|c|c|c|c|c|c|c|c|c|c|c|c|c|c|}
\hline 11TQ03-060 & 0.0651 & 0.0011 & 0.8402 & 0.0134 & 0.0936 & 0.0008 & 0.0311 & 0.0003 & 1.03 & 778 & 20 & 619 & 7 & 577 & 5 & 619 & 5 & 577 & 10 \\
\hline 11TQ03-061 & 0.1004 & 0.0011 & 4.0829 & 0.0379 & 0.2951 & 0.0023 & 0.0893 & 0.0006 & 1.25 & 1631 & 8 & 1651 & 8 & 1667 & 11 & 1730 & 12 & 1631 & 16 \\
\hline 1TQ03-062 & 0.0490 & 0.0024 & 0.0465 & .0023 & .0069 & 0.0001 & .0022 & 0.0000 & 1.63 & 149 & 113 & 46 & 2 & 44.2 & 0.5 & 44 & 0.4 & 44.2 & 1 \\
\hline 11TQ03-063 & 0.0513 & 0.0050 & 0.0595 & 0.0057 & 0.0084 & 0.0002 & 0.0027 & 0.0001 & 0.87 & 256 & 174 & 59 & 5 & 54 & 1 & 54 & 2 & 54 & 2 \\
\hline 1TQ03-064 & 0.0461 & 0.0027 & 0.0488 & 0.0028 & .0077 & 0.0001 & 0.0025 & 0.0001 & 1.87 & 1 & 126 & 48 & 3 & 49.3 & 0.6 & 50 & 2 & 49.3 & 1.2 \\
\hline ITQ03-065 & 0.0514 & .0133 & 0.1353 & .0342 & .0191 & 0.0011 & .0071 & 0.0007 & 1.14 & 257 & 380 & 129 & 31 & 122 & 7 & 144 & 14 & 122 & 14 \\
\hline 1TQ03-066 & 0.0653 & 0.0077 & 0.9425 & 0.1084 & 0.1047 & 0.0033 & .0356 & 0.0020 & 1.31 & 784 & 190 & 674 & 57 & 642 & 19 & 706 & 39 & 642 & 38 \\
\hline 1TQ03-067 & 0.1840 & 0.0024 & 12.9058 & 0.1343 & 0.5086 & 0.0042 & 0.1398 & 0.0012 & 2.07 & 2690 & 22 & 2673 & 10 & 2651 & 18 & 2645 & 20 & 2690 & 44 \\
\hline 1TQ03-068 & 0.0610 & 0.0010 & 0.7795 & 0.0110 & 0.0927 & .0008 & .0297 & 0.0003 & 1.29 & 641 & 17 & 585 & 6 & 571 & 4 & 591 & 5 & 571 & 8 \\
\hline 1TQ03-069 & 0.0844 & 0.0015 & 2.5284 & 0.0417 & 0.2174 & 0.0020 & .0656 & 0.0007 & 1.05 & 1301 & 18 & 1280 & 12 & 1268 & 11 & 1284 & 13 & 1301 & 36 \\
\hline 1TQ03-070 & 0.0483 & 0.0117 & 0.0894 & 0.0211 & 0.0134 & 0.0007 & 0.0050 & 0.0004 & 0.86 & 114 & 340 & 87 & 20 & 86 & 4 & 101 & 8 & 86 & 8 \\
\hline 11TQ03-071 & 0.0498 & 0.0054 & 0.1088 & 0.0115 & 0.0159 & 0.0004 & 0.0052 & 0.0002 & 1.06 & 184 & 191 & 105 & 11 & 101 & 3 & 105 & 4 & 101 & 6 \\
\hline 1TQ03-072 & 0.0494 & 0.0076 & 0.0647 & 0.0098 & 0.0095 & 0.0002 & 0.0030 & 0.0001 & 1.13 & 166 & 308 & 64 & 9 & 61 & 1 & 61 & 2 & 61 & 2 \\
\hline 1TQ03-073 & 0.0714 & 0.0015 & 1.5843 & 0.0300 & 0.1610 & 0.0015 & 0.0507 & 0.0008 & 3.03 & 969 & 24 & 964 & 12 & 962 & 8 & 1000 & 16 & 962 & 16 \\
\hline 11TQ03-074 & 0.0495 & 0.0144 & 0.1129 & 0.0321 & 0.0166 & 0.0010 & 0.0059 & 0.0006 & 1.24 & 171 & 410 & 109 & 29 & 106 & 6 & 120 & 13 & 106 & 12 \\
\hline 11TQ03-075 & 0.0505 & 0.0037 & 0.1503 & 0.0108 & 0.0216 & 0.0004 & 0.0082 & 0.0003 & 1.52 & 217 & 130 & 142 & 10 & 138 & 2 & 165 & 5 & 138 & 4 \\
\hline 11TQ03-076 & 0.0664 & 0.0017 & 1.2726 & 0.0295 & 0.1389 & 0.0012 & 0.0424 & 0.0003 & 1.71 & 820 & 53 & 834 & 13 & 839 & 7 & 839 & 6 & 839 & 14 \\
\hline 11TQ03-077 & 0.0523 & 0.0043 & 0.0814 & 0.0067 & 0.0113 & 0.0002 & 0.0035 & 0.0000 & 0.51 & 300 & 190 & 79 & 6 & 72.3 & 0.9 & 71.5 & 0.5 & 72.3 & 1.8 \\
\hline 11TQ03-078 & 0.0461 & 0.0059 & 0.0623 & 0.0078 & 0.0098 & 0.0002 & 0.0032 & 0.0002 & 1.01 & & 243 & 61 & 7 & 63 & 1 & 65 & 4 & 63 & 2 \\
\hline 11TQ03-079 & 0.0504 & 0.0035 & 0.1018 & 0.0069 & 0.0146 & 0.0003 & 0.0045 & 0.0001 & 0.91 & 214 & 124 & 98 & 6 & 94 & 2 & 90 & 2 & 94 & 4 \\
\hline 11TQ03-080 & 0.0514 & 0.0049 & 0.1045 & 0.0098 & 0.0147 & 0.0003 & 0.0053 & 0.0003 & 1.92 & 260 & 168 & 101 & 9 & 94 & 2 & 107 & 5 & 94 & 4 \\
\hline
\end{tabular}

2010TQ04 (Qiuwu, Qiabulin section, $\mathrm{n}=104, \mathrm{~N}^{\prime} 9^{\circ} 20.161^{\prime} \quad \mathrm{E88^{ \circ }} 30.669^{\prime}, 4072 \mathrm{~m}$ )

$\begin{array}{llllllllllllllllllll}\text { 10TQ04-001 } & 0.0557 & 0.0028 & 0.4420 & 0.0211 & 0.0576 & 0.0008 & 0.0190 & 0.0006 & 2.31 & 439 & 83 & 372 & 15 & 361 & 5 & 380 & 12 & 361 & 10\end{array}$ $\begin{array}{lllllllllllllllllllll}\text { 10TQ04-002 } & 0.0602 & 0.0011 & 0.8261 & 0.0128 & 0.0995 & 0.0007 & 0.0296 & 0.0003 & 1.00 & 611 & 21 & 611 & 7 & 612 & 4 & 589 & 5 & 612 & 8\end{array}$ $\begin{array}{llllllllllllllllllll}\text { 10TQ04-003 } & 0.0496 & 0.0017 & 0.1433 & 0.0047 & 0.0210 & 0.0002 & 0.0065 & 0.0001 & 0.64 & 176 & 59 & 136 & 4 & 134 & 1 & 131 & 1 & 134 & 2\end{array}$

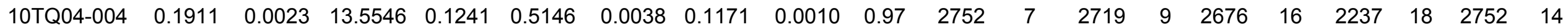
$\begin{array}{llllllllllllllllllll}\text { 10TQ04-005 } & 0.0466 & 0.0081 & 0.0551 & 0.0095 & 0.0086 & 0.0002 & 0.0028 & 0.0002 & 1.21 & 28 & 283 & 54 & 9 & 55 & 1 & 56 & 3 & 55 & 2\end{array}$ $\begin{array}{llllllllllllllllllll}\text { 10TQ04-006 } & 0.0478 & 0.0023 & 0.0247 & 0.0012 & 0.0038 & 0.0000 & 0.0012 & 0.0000 & 1.32 & 88 & 85 & 25 & 1 & 24.2 & 0.3 & 25 & 0.6 & 24.2 & 0.6\end{array}$ $\begin{array}{lllllllllllllllllllll}\text { 10TQ04-007 } & 0.0799 & 0.0019 & 2.2072 & 0.0489 & 0.2004 & 0.0019 & 0.0604 & 0.0007 & 0.77 & 1194 & 29 & 1183 & 15 & 1178 & 10 & 1185 & 14 & 1194 & 58\end{array}$

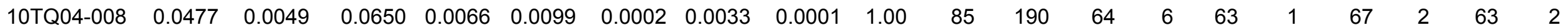

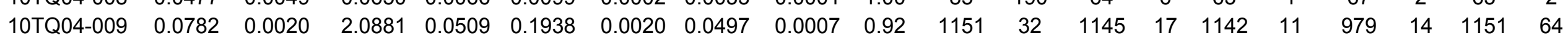
$\begin{array}{llllllllllllllllllllll}\text { 10TQ04-010 } & 0.0580 & 0.0007 & 0.6863 & 0.0069 & 0.0858 & 0.0006 & 0.0268 & 0.0002 & 3.04 & 530 & 11 & 531 & 4 & 531 & 3 & 535 & 4 & 531 & 6\end{array}$ $\begin{array}{llllllllllllllllllll}\text { 10TQ04-011 } & 0.0759 & 0.0012 & 1.9383 & 0.0251 & 0.1852 & 0.0013 & 0.0603 & 0.0008 & 3.23 & 1093 & 15 & 1094 & 9 & 1095 & 7 & 1183 & 14 & 1093 & 30\end{array}$ 


\begin{tabular}{|c|c|c|c|c|c|c|c|c|c|c|c|c|c|c|c|c|c|c|c|}
\hline 0TQ04-012 & .0804 & 0.0010 & 2.2755 & 0.0224 & 0.2054 & 0.0014 & 0.0621 & 0.0005 & 2.39 & 1206 & 10 & 1205 & 7 & 1204 & 7 & 1218 & 10 & 1206 & 20 \\
\hline 10TQ04-013 & 0.0483 & 0.0028 & 0.0938 & 0.0053 & 0.0141 & 0.0002 & 0.0051 & 0.0001 & 1.21 & 112 & 102 & 91 & 5 & 90 & 1 & 103 & 2 & 90 & 2 \\
\hline OTQ04-014 & 0.0516 & 0.0036 & 0.1002 & 0.0069 & 0.0141 & 0.0002 & 0.0043 & 0.0001 & 0.58 & 266 & 134 & 97 & 6 & 90 & 1 & 86 & 2 & 90 & 2 \\
\hline 0TQ04-015 & 0.0586 & 0.0011 & 0.6546 & 0.0108 & 0.0811 & 0.0006 & 0.0248 & 0.0003 & 2.71 & 550 & 23 & 511 & 7 & 503 & 4 & 496 & 6 & 503 & \\
\hline 0TQ04-016 & 0.0518 & 0.0020 & 0.2186 & 0.0081 & 0.0306 & 0.0003 & 0.0092 & 0.0001 & 0.39 & 276 & 67 & 201 & 7 & 194 & 2 & 186 & 2 & 194 & \\
\hline TTQ4-017 & 0.0487 & 0.0027 & 0.0979 & 0.0052 & 0.0146 & 0.0002 & 0.0045 & 0.0001 & .86 & 134 & 98 & 95 & 5 & 93 & 1 & 90 & 3 & 93 & \\
\hline TTQ04-018 & 0.0482 & 0110 & 0.0527 & 0.0119 & 0.0079 & 0.0003 & 0.0025 & 0.0001 & 0.49 & 107 & 349 & 52 & 11 & 51 & 2 & 51 & 2 & 51 & \\
\hline TTQ04-019 & 0.0571 & 0012 & .6676 & 0.0129 & 0.0848 & .0007 & 0.0269 & 0.0002 & .86 & 496 & 29 & 519 & & 525 & 4 & 536 & 5 & 525 & \\
\hline TTQ04-020 & 0.0484 & 0011 & 0.0419 & 0.0009 & .0063 & 01 & 22 & 00 & 38 & 116 & 33 & 41.6 & 0.8 & 40.4 & 0.3 & 43.8 & 0.8 & 40.4 & \\
\hline DTQ04-021 & 0.0494 & 0087 & 0.0583 & 0.0101 & .0086 & 0002 & 0.0026 & 001 & 0.79 & 167 & 305 & 58 & 10 & 55 & 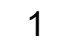 & 52 & 2 & 55 & \\
\hline OTQ04-022 & 0.1076 & .0014 & 4.7261 & 0.0508 & 3185 & 0.0023 & 0.0918 & 0.0008 & 1.53 & 1760 & 10 & 1772 & 9 & 1783 & 1 & 1774 & 6 & 760 & \\
\hline OTQ04-023 & 0.1773 & 0.0020 & 12.2701 & 0.1015 & .5021 & 0.0035 & 0.1430 & 0.0009 & 0.82 & 2628 & 6 & 2625 & 8 & 2623 & 15 & 2701 & $\gamma$ & 2628 & \\
\hline DTQ04-024 & 0.0705 & 0.0012 & 1.5229 & 0.0228 & 0.1567 & 0.0012 & 0.0478 & 0.0007 & 3.63 & 944 & 19 & 940 & 9 & 938 & 6 & 943 & 14 & 938 & \\
\hline 0TQ04-025 & 0.0475 & 0.0014 & 0.0527 & 0.0015 & 0.0081 & 0.0001 & 0.0025 & 0.0000 & 0.37 & 75 & 49 & 52 & 1 & 51.7 & 0.4 & 50.9 & 0.4 & 51.7 & \\
\hline OTQ04-026 & 0.1034 & 0.0012 & 4.3656 & 0.0345 & 0.3063 & 0.0020 & 0.0897 & 0.0006 & 1.27 & 1686 & 7 & 1706 & 7 & 1722 & 10 & 1737 & 10 & 1686 & \\
\hline OTQ04-027 & 0.0822 & 0.0016 & 2.3095 & 0.0415 & 0.2037 & 0.0018 & 0.0582 & 0.0008 & 1.47 & 1251 & 22 & 1215 & 13 & 1195 & 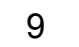 & 1143 & 15 & 1251 & \\
\hline-028 & 0.0585 & 0.0009 & 0.7013 & 0.0096 & 0.0869 & 0.0006 & 0.0264 & 0.0002 & 1.24 & 550 & 18 & 540 & 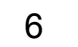 & 537 & 4 & 527 & 4 & 537 & \\
\hline 10TQ & 0.0480 & 0.0035 & 0.0989 & 0.0071 & 0.0150 & 0.0002 & 0.0024 & 0.0001 & 1.06 & 99 & 135 & 96 & 7 & 96 & 1 & 49 & 2 & 96 & \\
\hline 10TQ04-030 & 0.0608 & 0.0016 & 0.7695 & 0.0193 & 0.0918 & 0.0008 & 0.0286 & 0.0004 & 1.14 & 633 & 38 & 579 & 11 & 566 & 5 & 569 & 7 & 566 & 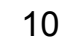 \\
\hline 4-031 & 0.1351 & 0.0036 & 6.8415 & 0.1768 & 0.3674 & 0.0052 & 0.1188 & 0.0021 & 0.75 & 2165 & 26 & 2091 & 23 & 2017 & 24 & 2268 & 37 & 2165 & 2 \\
\hline 0TQ04-032 & 0.0471 & 0.0089 & 0.0502 & 0.0093 & 0.0077 & 0.0003 & 0.0026 & 0.0002 & 0.73 & 56 & 278 & 50 & $y$ & 50 & 2 & 52 & 3 & 50 & \\
\hline-033 & 0.0749 & 0.0014 & 1.8597 & 0.0305 & $0.1 \varepsilon$ & 0.0014 & 0.0 & 11 & 4.23 & 1066 & 20 & 1067 & 11 & 1068 & 8 & 1162 & 20 & 1066 & 0 \\
\hline 10TQ04-034 & 0.0511 & 0.0127 & 0.1 & 0.0247 & 0.0143 & 0.0006 & 0.0055 & 05 & 1.47 & 246 & 384 & 98 & 23 & 92 & 4 & 110 & 10 & 92 & 8 \\
\hline 10TQ04-035 & 0.0644 & 0.0014 & 1.0991 & 0.0217 & 0.1239 & 0.0010 & 0.0 & 07 & 2.93 & 754 & 28 & 753 & 10 & 753 & 6 & 870 & 14 & 753 & 2 \\
\hline 10TC & 0.0 & 0.00 & & 0.0 & 0.03 & 0.0 & 0.0 & 03 & 1.55 & 221 & 109 & 225 & 12 & 226 & 3 & 241 & 6 & 226 & \\
\hline 10TC & 0.0 & 8 & & 0.02 & 0.0 & 06 & & 05 & 1.28 & 119 & 395 & 101 & 25 & 101 & 4 & 123 & 10 & 01 & \\
\hline $10 \mathrm{TC}$ & 0.0562 & 0047 & 13 & 0.0308 & 0.0479 & 0.0008 & 0.0152 & 06 & 1.65 & 461 & 155 & 321 & 23 & 302 & 5 & 305 & 12 & 302 & 0 \\
\hline-039 & 0.0516 & .0027 & 0.1907 & 0.0098 & 0.0268 & 0.0003 & 0.0083 & 0.0001 & 1.04 & 266 & 99 & 177 & & 17 & 2 & 167 & 3 & 171 & \\
\hline OTQO & 0.0479 & .0030 & 0.1048 & 0.0064 & 0.0159 & 0.0002 & 0.0052 & 0.0001 & 1.42 & 96 & 113 & 10 & 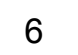 & 101 & 1 & 106 & 3 & 101 & \\
\hline OTQO & 0.0460 & .0017 & 0.0901 & 0.0032 & 0.0142 & 0.0001 & 0.0044 & 0.0001 & 1.33 & & 57 & 88 & 3 & 90.9 & 0.8 & 89 & 1 & 90.9 & 6 \\
\hline OTQ & 0.0461 & .0016 & 0.0842 & 0.0028 & 0.0133 & 0.0002 & 0.0048 & 0.0002 & 0.62 & & 74 & 82 & 3 & 84.9 & 1 & 96 & 4 & 84.9 & \\
\hline 0TQ04-043 & 0.0493 & 0.0051 & 0.0548 & 0.0056 & 0.0081 & 0.0001 & 0.0026 & 0.0001 & 0.87 & 161 & 197 & 54 & 5 & 51.7 & 0.9 & 53 & 2 & 51.7 & 8 \\
\hline-044 & 0.0493 & .0036 & 0.1336 & 0.0095 & 0.0197 & 0.0002 & 0.0067 & 0.0002 & 1.63 & 161 & 139 & 127 & 9 & 126 & 1 & 134 & 3 & 126 & \\
\hline 10TQ04-045 & 0.0471 & 0.0105 & 0.0513 & 0.0114 & 0.0079 & 0.0002 & 0.0027 & 0.0001 & 1.02 & 52 & 351 & 51 & 11 & 51 & 1 & 55 & 2 & 51 & \\
\hline
\end{tabular}




\begin{tabular}{|c|c|c|c|c|c|c|c|c|c|c|c|c|c|c|c|c|c|c|c|}
\hline 10TQ04-046 & .0916 & 0.0016 & 2.9488 & 0.0453 & 0.2335 & 0.0019 & 0.0747 & 0.0007 & 1.05 & 1459 & 17 & 1395 & 12 & 1353 & 10 & 1455 & 14 & 1459 & 34 \\
\hline OTQ04-047 & 0.0488 & 0.0084 & 0.1077 & 0.0185 & 0.0160 & 0.0003 & 0.0055 & 0.0002 & 1.06 & 136 & 296 & 104 & 17 & 102 & 2 & 112 & 5 & 102 & 1 \\
\hline 0TQ04-048 & 0.0576 & 0.0010 & 0.6656 & 0.0098 & 0.0838 & 0.0006 & 0.0304 & 0.0007 & 13.74 & 515 & 20 & 518 & 6 & 519 & 4 & 605 & 14 & 519 & 8 \\
\hline 0TQ04-049 & 0.0592 & 0.0010 & 0.7414 & 0.0110 & 0.0909 & 0.0006 & 0.0311 & 0.0007 & 12.89 & 574 & 20 & 563 & 6 & 561 & 4 & 620 & 15 & 561 & 3 \\
\hline 0TQ04-050 & 0.0588 & 0.0008 & 0.8064 & 0.0091 & 0.0995 & 0.0007 & 0.0307 & 0.0002 & 1.37 & 560 & 14 & 600 & 5 & 611 & 4 & 611 & 4 & 611 & \\
\hline 0TQ04-051 & 0.0494 & 0.0020 & 0.0580 & 0.0022 & 0.0085 & 0.0001 & 0.0029 & 0.0001 & 1.65 & 165 & 72 & 57 & 2 & 54.8 & 0.5 & 59 & 1 & 54.8 & \\
\hline OTQ04-052 & 0.1048 & 0.0013 & 4.1218 & 0.0388 & 0.2854 & 0.0019 & 0.0821 & 0.0007 & 2.24 & 1711 & 8 & 1659 & 8 & 1618 & 10 & 1595 & 14 & 1711 & 6 \\
\hline OTQ04-053 & 0.0987 & .0014 & 4.0106 & 0.0474 & 2947 & .0022 & 0.0858 & 0.0007 & 0.89 & 1600 & 12 & 1636 & 10 & 1665 & 11 & 663 & 13 & 1600 & 21 \\
\hline 54 & 0.0576 & 0013 & 0.6451 & 0.0134 & .0813 & 0.0007 & 0.0252 & 002 & 0.81 & 513 & 32 & 505 & & 504 & 4 & 503 & - & 504 & \\
\hline 55 & 0.0479 & 5 & .0914 & 0.0028 & 0139 & 1 & 43 & 0001 & 0.70 & 92 & 54 & 89 & & 8.7 & 0.8 & 87 & & 88.7 & \\
\hline 56 & 0678 & 12 & 1.1709 & .0175 & 1254 & 9 & 85 & 03 & 1.19 & 861 & 19 & 787 & & 761 & 5 & 763 & 6 & 761 & \\
\hline 57 & 0740 & 0009 & 1.7905 & .0153 & 1756 & .0011 & 25 & 03 & 1.76 & 1041 & & 1042 & & 1043 & 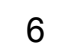 & 1035 & 6 & 1041 & \\
\hline 58 & 0.0474 & .0034 & 0.0222 & 0.0016 & .0034 & .0000 & 0.0010 & .0000 & 1.22 & 70 & 134 & 22 & & 21.9 & 0.3 & 21 & 0.4 & 21.9 & 6 \\
\hline 059 & 0.0528 & .0086 & 0.1258 & 0.0205 & .0173 & 0.0003 & 0.0045 & 0.0002 & 0.85 & 322 & 319 & 120 & 18 & 110 & ? & 91 & 5 & 110 & \\
\hline 060 & 0.0492 & .0044 & 0.0666 & 0.0058 & .0098 & 0.0002 & 0.0032 & 0.0001 & 1.32 & 158 & 167 & 66 & & 6 & 1 & 64 & 2 & 63 & \\
\hline OTQ04-061 & 0.0493 & 0.0093 & 0.0620 & 0.0116 & 0.0091 & .0003 & 0.0028 & 0.0001 & 0.67 & 163 & 310 & 61 & 11 & 59 & 2 & 5 & 3 & 59 & \\
\hline-062 & 0.0512 & 0.0084 & 0.1005 & 0.0161 & 0.0143 & 0.0004 & 0.0040 & 0.0002 & 0.94 & 250 & 287 & 97 & 5 & 91 & 3 & 81 & 5 & 91 & \\
\hline 063 & 0.0474 & 0.0104 & 0.0564 & 0.0122 & 0.0086 & 0.0003 & 0.0030 & 0.0002 & 1.04 & 68 & 331 & 56 & 12 & 55 & 2 & 61 & 4 & 55 & \\
\hline 64 & 0.1961 & 0.0025 & 13.7443 & 0.1435 & 0.5086 & 0.0041 & 0.1393 & 0.0011 & 0.64 & 279 & 8 & 2732 & 10 & 2651 & 18 & 2636 & 20 & 2794 & 0 \\
\hline 10TQ & 0.0494 & 0.0022 & 0.1078 & 0.0046 & 0.0158 & 0.0002 & 0.0048 & 0.0001 & 1.20 & 166 & 79 & 104 & 4 & 101 & 1 & 97 & 2 & 101 & 2 \\
\hline 10TQ04-066 & 0.1360 & 0.0015 & 7.4985 & 0.0535 & 0.4000 & 0.0025 & 0.1128 & 0.0007 & 1.73 & 2177 & 6 & 2173 & 6 & 2169 & 12 & 2160 & 12 & 2177 & 2 \\
\hline $10 \mathrm{TC}$ & 0.0715 & 0.0014 & 1.5356 & 0.0271 & 0.1559 & 0.0013 & 0.0438 & 0.0006 & 1.78 & 971 & 23 & 945 & 11 & 934 & 7 & 867 & 11 & 934 & 4 \\
\hline-068 & 0.0601 & 0.0024 & 0.6365 & 0.0250 & 0.0768 & 0.0008 & 0.0237 & 0.0002 & 3.83 & 608 & 90 & 500 & 15 & 477 & 5 & 474 & 4 & 477 & 0 \\
\hline-069 & 0.1564 & 0.0018 & 9.7428 & 0.0863 & 0.4519 & 0.0032 & 0.1221 & 0.0009 & 0.97 & 2417 & 7 & 2411 & 8 & 2404 & 14 & 2329 & 16 & 2417 & 4 \\
\hline 070 & 0.0469 & 0.0025 & 0.0550 & 0.0029 & 0.0085 & 0.0001 & & 01 & 0.86 & 42 & 92 & 54 & 2 & 54.7 & 0.6 & 51 & 1 & 4.7 & .2 \\
\hline $10 \mathrm{TC}$ & 0.0472 & 0.0 & 72 & 0.0026 & 0.0073 & & & 00 & 0.87 & 01 & 99 & 47 & 3 & 46.6 & 0.5 & 43.4 & 0.8 & 46.6 & \\
\hline $10 \mathrm{TC}$ & 0.07 & 1 & & 0.0424 & & & & & 2.38 & 937 & 42 & 921 & 17 & 915 & 9 & 879 & 20 & 15 & 8 \\
\hline 10TC & & & & 0.0068 & & & & 01 & 1.09 & 73 & 232 & 54 & 7 & 7 & 8 & 50 & 2 & .7 & 6 \\
\hline 74 & 0.0474 & .0020 & & 0.0019 & 0.0071 & 001 & 0.0019 & 0.0000 & 1.99 & 69 & 71 & 46 & 2 & 45.9 & 0.4 & 39 & 0.8 & 45.9 & \\
\hline 75 & 0.0702 & 0009 & & 0.0159 & 1565 & . 0010 & 0.0466 & 0.0004 & 2.00 & 93 & 11 & 936 & 6 & 93 & 6 & 920 & 7 & 937 & 12 \\
\hline 076 & 0.0481 & 0090 & .0540 & 0.0100 & .0081 & 0.0003 & 0.0031 & 0.0002 & 0.80 & 103 & 293 & 53 & 10 & 52 & 2 & 63 & 3 & 52 & \\
\hline & & & & 0.0034 & & & & 0.0000 & 0.39 & $19 \varepsilon$ & 64 & 93 & ? & 89.1 & 0.9 & 81.7 & 0.8 & 89.1 & \\
\hline 78 & 84 & .0050 & & 0.0054 & 0.0080 & 0.0001 & 0.0024 & 0.0001 & 0.76 & 116 & 197 & 53 & 5 & 51.4 & 0.8 & 49 & 1 & 51.4 & \\
\hline 10TQ04-079 & 0.0479 & 0.0030 & 0.0490 & 0.0030 & 0.0074 & 0.0001 & 0.0023 & 0.0000 & 0.80 & 96 & 116 & 49 & 3 & 47.7 & 0.5 & 46.8 & 0.8 & 47.7 & \\
\hline
\end{tabular}




\begin{tabular}{|c|c|c|c|c|c|c|c|c|c|c|c|c|c|c|c|c|c|c|c|}
\hline TQ04-080 & 0581 & .0015 & 0.6947 & 0.0165 & 0.0868 & .0007 & 0.0339 & 1.0019 & 4.18 & 532 & 37 & 36 & 10 & 37 & 4 & 673 & 37 & 37 & 8 \\
\hline 0TQ04-081 & 0.1043 & . 0016 & 3544 & 0.0591 & 0.3028 & 0024 & 0865 & .0008 & 0.80 & 1703 & 14 & & 11 & 705 & 12 & 1677 & 14 & 1703 & 28 \\
\hline JTQ04-082 & .0732 & .0011 & 7213 & 0.0211 & 0.1707 & 0012 & 0.0486 & 0005 & 1.91 & 19 & 14 & 017 & 0 & 16 & 7 & 959 & 0 & 19 & 28 \\
\hline TQ04-083 & 0.0467 & 0092 & 38 & 0.0105 & .0084 & .0003 & 0.0025 & 0001 & 0.68 & 22 & 299 & 53 & 10 & 5 & 2 & 49 & & 54 & 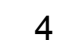 \\
\hline TQ04-084 & 0.0490 & 0025 & 0793 & .0039 & 8 & .0001 & 0.0035 & 0001 & 0. & 145 & 90 & 77 & & 75.4 & 0.9 & 71 & & 5.4 & 8 \\
\hline 35 & 0.09 & $O C$ & 6 & 2 & 1 & & & & & 14 & 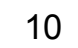 & & & 139 & 0 & 04 & 13 & 39 & 20 \\
\hline 6 & 0 & 00 & & 45 & & & & & & 92 & 22 & 2 & 10 & 893 & 7 & 4 & 1 & 93 & 14 \\
\hline 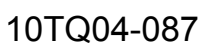 & 0488 & .00 & 5 & 27 & 45 & 01 & & & 0.60 & 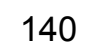 & 5 & 9 & & Y. & 0.0 & 87.5 & 0.8 & 02.1 & 1.6 \\
\hline 8 & 8 & 22 & 8 & 3 & 46 & 2 & & & 1.0 & 88 & & y & & & & 85 & & 93 & 2 \\
\hline 9 & 0486 & 39 & 3 & 76 & 0145 & 2 & & & .91 & 1 & 144 & 9 & & & & & & 93 & 4 \\
\hline 30 & 0763 & 12 & 81 & .0268 & 61 & 4 & 38 & 5 & 1.62 & 110 & 1 & 1101 & & 11 & & 059 & 0 & 3 & 32 \\
\hline 1 & 0498 & 20 & 13 & .0057 & .0220 & 02 & 64 & 01 & 0.7 & 18 & & 14 & & 14 & & 12 & & 14 & 2 \\
\hline 2 & 0479 & 0054 & .0559 & .0062 & .0085 & 002 & 0.0026 & 01 & .4 & 96 & 20 & 5 & & 5 & & 52 & & 54 & 2 \\
\hline 93 & 0593 & 0014 & 0.7480 & .0159 & .0915 & 0007 & 0.0264 & 003 & 1.2 & 57 & 32 & 56 & 9 & 56 & 4 & 526 & 6 & 65 & 8 \\
\hline 4 & 0476 & .0039 & 0.0514 & 0.0041 & 0.0078 & 101 & 0.0021 & 01 & 0.4 & 78 & 14 & 5 & 4 & 50.3 & 0.8 & 43 & 1 & 50.3 & 1.6 \\
\hline 95 & 0718 & .0011 & 1.6503 & 0.0202 & 0.1667 & 12 & 0.0 & 05 & 2.80 & 98 & 14 & $9 s$ & $\varepsilon$ & $9 s$ & 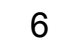 & 927 & 10 & 994 & 12 \\
\hline 36 & 0766 & 2 & 1.9672 & 0.0250 & 1862 & 3 & 96 & 66 & 80.18 & 1112 & 1 & 110 & 5 & 1101 & & 2817 & 116 & 1112 & 28 \\
\hline 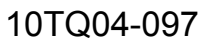 & 0479 & 3 & .0965 & 0.0046 & .0146 & 02 & 7 & 01 & 1.13 & 9 & 8 & 94 & 4 & 94 & & 95 & 2 & 94 & 2 \\
\hline 88 & 0485 & 0.0028 & 0525 & .0029 & .0079 & 0.0001 & 26 & 01 & 0.90 & 12 & 10 & 52 & & 50.4 & 0 & 51 & 1 & 50.4 & 1.2 \\
\hline 999 & 0466 & 0074 & .0542 & 0.0086 & .0084 & 002 & 0.0028 & 001 & .86 & 30 & 259 & 5 & 8 & 54 & 1 & 56 & 2 & 54 & 2 \\
\hline 0 & 2583 & .0027 & 23.5274 & 0.1706 & .6607 & 0.0044 & 0.1520 & 0.0012 & 2.23 & 3237 & 5 & 3249 & $t$ & 3270 & 17 & 2860 & 22 & 3237 & 10 \\
\hline 101 & 0.0591 & .0016 & 0.7273 & 0.0180 & 0.0894 & 0.0008 & 0.0266 & 0.0004 & 1.62 & 569 & 39 & 555 & 11 & 552 & 5 & 530 & 7 & 552 & 10 \\
\hline $04-102$ & 0.0484 & 0117 & 0.1235 & 0.0296 & 0.0185 & 0.0006 & 0.0054 & 0.0005 & 1.34 & 117 & 374 & 118 & 27 & 118 & 4 & 109 & 10 & 18 & 8 \\
\hline & 0.0812 & 0.0020 & 2.3225 & 0.0528 & 0.2075 & 0.0020 & 0.0595 & 0.0007 & 0.78 & 1226 & 29 & 1219 & 16 & 1216 & 11 & 1167 & 14 & 1226 & 58 \\
\hline TQ04-104 & 0.0499 & 0.0029 & 0.1045 & 0.0059 & 0.0152 & 0.0002 & 0.0049 & 0.0001 & 1.66 & 191 & 105 & 101 & 5 & 97 & 1 & 99 & 3 & 97 & 2 \\
\hline
\end{tabular}

$2010 T Q 06$ (Qiuwu, Qiabulin section, $\mathrm{n}=105, \mathrm{~N}^{\circ} 9^{\circ} 20.132^{\prime} \mathrm{E}^{\circ} 8^{\circ} 30.658^{\prime}, 4100 \mathrm{~m}$ )

$\begin{array}{lllllllllllllllllllll}\text { 10TQ06-001 } & 0.0489 & 0.0044 & 0.0231 & 0.0021 & 0.0034 & 0.0001 & 0.0011 & 0.0000 & 0.34 & 142 & 167 & 23 & 2 & 22.1 & 0.4 & 22.6 & 0.4 & 22.1 & 0.8\end{array}$

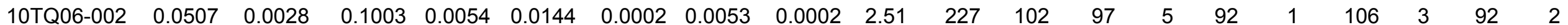
$\begin{array}{llllllllllllllllllll}\text { 10TQ06-003 } & 0.0986 & 0.0015 & 3.7848 & 0.0471 & 0.2783 & 0.0021 & 0.0893 & 0.0008 & 1.13 & 1599 & 12 & 1590 & 10 & 1583 & 11 & 1729 & 15 & 1599 & 24\end{array}$

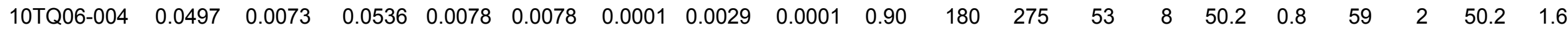

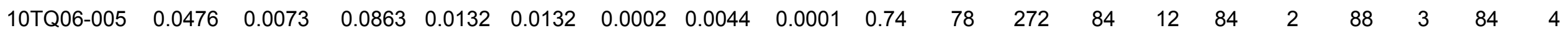
$\begin{array}{lllllllllllllllllllll}\text { 10TQ06-006 } & 0.0687 & 0.0009 & 1.3554 & 0.0147 & 0.1431 & 0.0010 & 0.0473 & 0.0004 & 1.89 & 890 & 12 & 870 & 6 & 862 & 5 & 935 & 7 & 862 & 10\end{array}$ $\begin{array}{lllllllllllllllllllll}10 T Q 06-007 & 0.0510 & 0.0111 & 0.0586 & 0.0127 & 0.0083 & 0.0002 & 0.0030 & 0.0001 & 0.41 & 241 & 366 & 58 & 12 & 53 & 1 & 61 & 2 & 53 & 2\end{array}$ 
10TQ06-008 $0.0513 \quad 0.0037$ 10TQ06-009 $0.0533 \quad 0.0011$ 10TQ06-010 $0.0510 \quad 0.0015$ 10TQ06-011 $0.0480 \quad 0.0057$

$\begin{array}{lll}\text { 10TQ06-012 } & 0.0867 & 0.0011\end{array}$ $\begin{array}{lll}10 T Q 06-013 & 0.0790 & 0.0013\end{array}$

$\begin{array}{lll}\text { 10TQ06-014 } & 0.0577 & 0.0019\end{array}$ 10TQ06-015 $0.1569 \quad 0.0033$

10TQ06-016 $0.0569 \quad 0.0008$

10TQ06-017 $0.0548 \quad 0.0012$

$\begin{array}{lll}\text { 10TQ06-018 } & 0.1219 & 0.0021\end{array}$

10TQ06-019 $0.0481 \quad 0.0037$

10TQ06-020 $0.0632 \quad 0.0056$

$\begin{array}{lll}\text { 10TQ06-021 } & 0.1096 & 0.0013\end{array}$

$\begin{array}{lll}\text { 10TQ06-022 } & 0.0494 & 0.0057\end{array}$

$\begin{array}{lll}\text { 10TQ06-023 } & 0.0469 & 0.0017\end{array}$

$\begin{array}{lll}\text { 10TQ06-024 } & 0.0478 & 0.0084\end{array}$

$\begin{array}{lll}\text { 10TQ06-025 } & 0.0467 & 0.0206\end{array}$

10TQ06-026 $0.0586 \quad 0.0016$

10TQ06-027 $0.0519 \quad 0.0010$

10TQ06-028 $0.0499 \quad 0.0055$

10TQ06-029 $0.0505 \quad 0.0031$

10TQ06-030 $0.0507 \quad 0.0029$

$\begin{array}{lll}\text { 10TQ06-031 } & 0.0486 & 0.0030\end{array}$

10TQ06-032 $0.1565 \quad 0.0022$

$\begin{array}{lll}\text { 10TQ06-033 } & 0.0500 & 0.0063\end{array}$

$\begin{array}{lll}\text { 10TQ06-034 } & 0.0490 & 0.0061\end{array}$

$\begin{array}{lll}\text { 10TQ06-035 } & 0.0486 & 0.0014\end{array}$

$\begin{array}{lll}\text { 10TQ06-036 } & 0.0496 & 0.0017\end{array}$

$\begin{array}{lll}10 T Q 06-037 & 0.0538 & 0.0011\end{array}$

$\begin{array}{lll}\text { 10TQ06-038 } & 0.0480 & 0.0033\end{array}$

$\begin{array}{lll}\text { 10TQ06-039 } & 0.0477 & 0.0036\end{array}$

10TQ06-040 $0.0494 \quad 0.0124$

$\begin{array}{lll}10 T Q 06-041 & 0.0485 & 0.0045\end{array}$ $\begin{array}{lllllll}0.1590 & 0.0113 & 0.0225 & 0.0004 & 0.0084 & 0.0003 & 1.46\end{array}$ $\begin{array}{llllllll}0.3943 & 0.0073 & 0.0537 & 0.0004 & 0.0175 & 0.0005 & 10.37\end{array}$ $\begin{array}{lllllll}0.0504 & 0.0014 & 0.0072 & 0.0001 & 0.0027 & 0.0001 & 2.53\end{array}$ $\begin{array}{lllllll}0.1107 & 0.0131 & 0.0167 & 0.0003 & 0.0059 & 0.0002 & 1.40\end{array}$ $\begin{array}{lllllll}2.6919 & 0.0272 & 0.2253 & 0.0016 & 0.0728 & 0.0006 & 1.42\end{array}$ $\begin{array}{lllllll}2.1327 & 0.0315 & 0.1959 & 0.0015 & 0.0624 & 0.0005 & 0.77\end{array}$ $\begin{array}{llllllll}0.6708 & 0.0207 & 0.0843 & 0.0007 & 0.0266 & 0.0005 & 1.62\end{array}$ $\begin{array}{llllllll}9.4967 & 0.1813 & 0.4391 & 0.0039 & 0.1226 & 0.0010 & 0.61\end{array}$ $\begin{array}{llllllll}0.6114 & 0.0072 & 0.0779 & 0.0005 & 0.0264 & 0.0002 & 0.98\end{array}$ $\begin{array}{llllllll}0.4057 & 0.0077 & 0.0538 & 0.0004 & 0.0168 & 0.0005 & 10.30\end{array}$ $\begin{array}{lllllll}6.1483 & 0.0955 & 0.3658 & 0.0029 & 0.1047 & 0.0008 & 1.47\end{array}$ $\begin{array}{llllllll}0.0781 & 0.0060 & 0.0118 & 0.0001 & 0.0038 & 0.0001 & 0.76\end{array}$ $\begin{array}{lllllll}0.7794 & 0.0679 & 0.0895 & 0.0020 & 0.0323 & 0.0019 & 2.31\end{array}$ $\begin{array}{lllllll}4.8485 & 0.0407 & 0.3209 & 0.0022 & 0.1007 & 0.0009 & 3.84\end{array}$ $\begin{array}{llllllll}0.0273 & 0.0031 & 0.0040 & 0.0001 & 0.0023 & 0.0001 & 2.76\end{array}$ $\begin{array}{llllllll}0.0880 & 0.0030 & 0.0136 & 0.0001 & 0.0045 & 0.0000 & 0.52\end{array}$ $\begin{array}{lllllll}0.0522 & 0.0091 & 0.0079 & 0.0002 & 0.0024 & 0.0001 & 0.82\end{array}$ $\begin{array}{lllllll}0.0483 & 0.0212 & 0.0075 & 0.0003 & 0.0023 & 0.0004 & 1.38\end{array}$ $\begin{array}{llllllll}0.7056 & 0.0181 & 0.0873 & 0.0008 & 0.0279 & 0.0003 & 0.43\end{array}$ $\begin{array}{lllllll}0.3839 & 0.0067 & 0.0536 & 0.0004 & 0.0175 & 0.0004 & 10.45\end{array}$ $\begin{array}{lllllll}0.0614 & 0.0066 & 0.0089 & 0.0002 & 0.0033 & 0.0001 & 1.22\end{array}$ $\begin{array}{lllllll}0.0522 & 0.0031 & 0.0075 & 0.0001 & 0.0026 & 0.0001 & 0.72\end{array}$ $\begin{array}{llllllll}0.0515 & 0.0028 & 0.0074 & 0.0001 & 0.0030 & 0.0001 & 2.44\end{array}$ $\begin{array}{llllllll}0.1014 & 0.0060 & 0.0152 & 0.0002 & 0.0006 & 0.0002 & 1.12\end{array}$ $\begin{array}{llllllll}9.7219 & 0.1161 & 0.4505 & 0.0033 & 0.1258 & 0.0009 & 1.13\end{array}$ $\begin{array}{llllllll}0.0537 & 0.0067 & 0.0078 & 0.0001 & 0.0032 & 0.0002 & 1.64\end{array}$ $\begin{array}{llllllll}0.0493 & 0.0060 & 0.0073 & 0.0001 & 0.0025 & 0.0001 & 0.67\end{array}$ $\begin{array}{llllllll}0.0782 & 0.0021 & 0.0117 & 0.0001 & 0.0039 & 0.0001 & 1.33\end{array}$ $\begin{array}{lllllll}0.0429 & 0.0014 & 0.0063 & 0.0001 & 0.0021 & 0.0001 & 2.93\end{array}$ $\begin{array}{llllllll}0.3979 & 0.0071 & 0.0536 & 0.0004 & 0.0173 & 0.0004 & 10.38\end{array}$ $\begin{array}{llllllll}0.0968 & 0.0065 & 0.0146 & 0.0002 & 0.0043 & 0.0001 & 0.92\end{array}$ $\begin{array}{lllllll}0.0533 & 0.0040 & 0.0081 & 0.0001 & 0.0025 & 0.0001 & 0.89\end{array}$ $\begin{array}{llllllll}0.0535 & 0.0132 & 0.0079 & 0.0003 & 0.0029 & 0.0002 & 0.92\end{array}$ $\begin{array}{lllllll}0.0866 & 0.0079 & 0.0130 & 0.0002 & 0.0042 & 0.0001 & 1.01\end{array}$

$\begin{array}{cccccccccc}253 & 133 & 150 & 10 & 143 & 2 & 170 & 5 & 143 & 4 \\ 340 & 29 & 337 & 5 & 337 & 2 & 350 & 9 & 337 & 4 \\ 239 & 49 & 50 & 1 & 46.1 & 0.4 & 55 & 1 & 46.1 & 0.8 \\ 100 & 230 & 107 & 12 & 107 & 2 & 119 & 5 & 107 & 4 \\ 1353 & 10 & 1326 & 7 & 1310 & 8 & 1419 & 10 & 1353 & 20 \\ 1172 & 17 & 1159 & 10 & 1153 & 8 & 1224 & 10 & 1172 & 34 \\ 520 & 52 & 521 & 13 & 522 & 4 & 530 & 9 & 522 & 8 \\ 2422 & 37 & 2387 & 18 & 2346 & 17 & 2337 & 18 & 2422 & 74 \\ 488 & 15 & 484 & 5 & 484 & 3 & 527 & 3 & 484 & 6 \\ 402 & 29 & 346 & 6 & 338 & 3 & 337 & 9 & 338 & 6 \\ 1984 & 32 & 1997 & 14 & 2010 & 14 & 2012 & 14 & 1984 & 64 \\ 103 & 147 & 76 & 6 & 75.6 & 0.9 & 77 & 2 & 75.6 & 1.8 \\ 714 & 147 & 585 & 39 & 553 & 12 & 643 & 37 & 553 & 24 \\ 1793 & 7 & 1793 & 7 & 1794 & 10 & 1938 & 17 & 1793 & 14 \\ 166 & 226 & 27 & 3 & 25.8 & 0.4 & 46 & 2 & 25.8 & 0.8 \\ 45 & 60 & 86 & 3 & 87.1 & 0.7 & 89.9 & 0.8 & 87.1 & 1.4 \\ 91 & 291 & 52 & 9 & 51 & 1 & 48 & 2 & 51 & 2 \\ 35 & 675 & 48 & 21 & 48 & 2 & 46 & 8 & 48 & 4 \\ 553 & 40 & 542 & 11 & 540 & 5 & 555 & 5 & 540 & 10 \\ 282 & 26 & 330 & 5 & 337 & 2 & 350 & 8 & 337 & 4 \\ 189 & 205 & 61 & 6 & 57 & 1 & 66 & 3 & 57 & 2 \\ 218 & 112 & 52 & 3 & 48.2 & 0.6 & 52 & 1 & 48.2 & 1.2 \\ 227 & 102 & 51 & 3 & 47.3 & 0.6 & 61 & 2 & 47.3 & 1.2 \\ 127 & 110 & 98 & 6 & 97 & 1 & 13 & 3 & 97 & 2 \\ 2418 & 24 & 2409 & 11 & 2397 & 14 & 2395 & 16 & 2418 & 48 \\ 193 & 249 & 53 & 6 & 50.1 & 0.8 & 65 & 3 & 50.1 & 1.6 \\ 146 & 237 & 49 & 6 & 46.9 & 0.9 & 50 & 2 & 46.9 & 1.8 \\ 130 & 48 & 76 & 2 & 74.7 & 0.6 & 78 & 1 & 74.7 & 1.2 \\ 175 & 60 & 43 & 1 & 40.4 & 0.4 & 43 & 1 & 40.4 & 0.8 \\ 364 & 27 & 340 & 5 & 337 & 2 & 346 & 9 & 337 & 4 \\ 101 & 125 & 94 & 6 & 94 & 1 & 87 & 2 & 94 & 2 \\ 85 & 139 & 53 & 4 & 51.9 & 0.7 & 50 & 1 & 51.9 & 1.4 \\ 168 & 378 & 53 & 13 & 50 & 2 & 59 & 4 & 50 & 4 \\ 122 & 171 & 84 & 7 & 83 & 1 & 86 & 3 & 83 & 2\end{array}$




\begin{tabular}{|c|c|c|c|c|c|c|c|c|c|c|c|c|c|c|c|c|c|c|c|}
\hline 10TQ06-042 & .0501 & 0.0028 & 0.1010 & 0.0055 & 0.0146 & 0.0002 & 0.0047 & 0.0001 & 0.60 & 197 & 101 & 98 & 5 & 94 & 1 & 94 & 2 & 94 & 2 \\
\hline 0TQ06-043 & .0491 & 0.0045 & 0.0552 & 0.0049 & 0.0082 & 0.0001 & 0.0026 & 0.0001 & 0.37 & 152 & 170 & 55 & 5 & 52.4 & 0.8 & 52 & 1 & 52.4 & 1.6 \\
\hline 0TQ06-044 & .0486 & 0.0071 & 0.0681 & 0.0098 & 0.0102 & 0.0003 & 0.0040 & 0.0002 & 1.50 & 130 & 255 & 67 & 9 & 65 & 2 & 80 & 4 & 65 & 4 \\
\hline 0TQ06-045 & 0.0484 & 0.0039 & 0.0421 & 0.0033 & 0.0063 & 0.0001 & 0.0016 & 0.0001 & 0.89 & 121 & 148 & 42 & 3 & 40.5 & 0.6 & 33 & 1 & 40.5 & 1.2 \\
\hline OTQ06-046 & .0498 & 0.0187 & 0.0531 & 0.0198 & 0.0077 & 0.0003 & 0.0027 & 0.0002 & 0.47 & 188 & 575 & 53 & 19 & 50 & 2 & 55 & 3 & 50 & 4 \\
\hline OTQ06-047 & .0534 & 0.0010 & 0.3954 & 0.0068 & 0.0537 & 0.0004 & 0.0168 & 0.0004 & 10.29 & 345 & 26 & 338 & 5 & 337 & 2 & 338 & $y$ & 337 & 4 \\
\hline JTQ06-048 & .0780 & 0.0011 & 2.0551 & 0.0239 & 0.1913 & 0.0014 & 0.0622 & 0.0006 & 2.27 & 1146 & 12 & 1134 & 8 & 1128 & 7 & 1219 & 12 & 1146 & 24 \\
\hline OTQ06-049 & .0489 & 0.0015 & 0.0407 & 0.0012 & 0.0 & 0.0001 & 0.0020 & 0.0000 & 1.46 & 145 & 54 & 41 & 1 & 38.8 & 0.3 & 9.8 & 0.6 & 38.8 & 0.6 \\
\hline 50 & 1223 & 0.0014 & 6.0 & 0.0532 & 0.3 & 0.0025 & 0.1020 & 0.0007 & 0.97 & 990 & 7 & 983 & 8 & 1977 & 12 & 963 & 13 & 90 & 14 \\
\hline 051 & 0777 & 0.0011 & 2.0304 & .0246 & 96 & .0014 & 0.0592 & 0.0005 & 1.35 & 139 & 13 & 126 & 0 & 1119 & 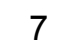 & 162 & 10 & 139 & 26 \\
\hline 052 & 0801 & 0.0024 & 2.1838 & .0631 & 78 & .0021 & 0.0484 & 008 & 0.89 & 199 & 40 & 1176 & 20 & 1163 & 11 & 95 & 15 & 1199 & 80 \\
\hline 053 & .0770 & .0011 & 1.7582 & 0200 & 56 & .0012 & 0.0615 & 06 & 2.82 & 122 & 12 & 1030 & & 988 & & 206 & 11 & 988 & 12 \\
\hline 054 & .0714 & 0.0010 & 1.5714 & .0173 & 0.1596 & .0011 & 0.0515 & 0.0005 & 3.28 & 969 & 12 & 95 & & 95 & & 1015 & 10 & 955 & 12 \\
\hline 055 & .0531 & 0.0010 & 0.3934 & 0.0068 & 0.0537 & 0.0004 & 0.0172 & 0.0004 & 10.21 & 335 & 26 & 337 & 5 & 337 & 2 & 345 & 8 & 337 & 4 \\
\hline-056 & 1039 & 0.0013 & 4.3487 & 0.0446 & 0.3037 & 0.0022 & 0.0920 & 0.0008 & 1.81 & 1694 & 9 & 1703 & 8 & 1710 & 11 & 1778 & 15 & 1694 & 18 \\
\hline JTQ06-057 & 0.0503 & 0.0061 & 0.0491 & 0.0058 & 0.0071 & 0.0002 & 0.0026 & 0.0001 & 0.86 & 209 & 226 & 49 & 6 & 45 & 1 & 53 & 2 & 45 & 2 \\
\hline-058 & .0464 & 0.0057 & 0.0265 & 0.0032 & 0.0041 & 0.0001 & 0.0014 & 0.0001 & 2.07 & 17 & 216 & 27 & 3 & 26.6 & 0.5 & 27 & 2 & 26.6 & 1 \\
\hline 059 & 0.0472 & 0.0021 & 0.0932 & 0.0040 & 0.0143 & 0.0002 & 0.0046 & 0.0001 & 1.12 & 5 & 74 & 90 & 4 & 91.7 & 1 & 93 & 2 & 91.7 & 2 \\
\hline 060 & .0531 & 0.0018 & 0.2439 & 0.0078 & 0.0333 & 0.0003 & 0.0105 & 0.0001 & 0.48 & 333 & 55 & 222 & 6 & 211 & 2 & 212 & 2 & 211 & 4 \\
\hline-061 & .0483 & 0.0074 & 0.0540 & .0081 & 0.0081 & 0.0003 & 0.0026 & 0.0001 & 0.70 & 113 & 255 & 53 & & 52 & 2 & 53 & 2 & 52 & 4 \\
\hline 062 & 0.1609 & 0.0019 & 10.3043 & 0.0909 & 0.4647 & 0.0033 & 0.1326 & 0.0009 & 0.56 & 2465 & 7 & 2462 & 8 & 2460 & 15 & 2516 & 15 & 2465 & 14 \\
\hline-063 & 0.0486 & 0.0038 & 0.0984 & 0.0075 & 0.0147 & 0.0002 & 0.0048 & 0.0001 & 0.84 & 131 & 140 & 95 & 7 & 94 & 1 & 97 & 2 & 94 & 2 \\
\hline-064 & 0.0488 & 0.0080 & 0.1075 & 0.0174 & 0.0160 & 0.0003 & 0.0065 & 0.0003 & 1.17 & 140 & 296 & 104 & 16 & 102 & 2 & 131 & 5 & 102 & 4 \\
\hline-065 & 0.0527 & 0.0010 & 0.3901 & 0.0064 & 0.0537 & 0.0004 & 0.0177 & 0.0005 & 10.46 & 317 & 24 & 334 & 5 & 337 & 2 & 354 & 9 & 337 & 4 \\
\hline 066 & 0.0482 & 0.0017 & 0.0449 & 0.0016 & 0.0068 & 0.0001 & 0.0014 & 0.0001 & 4.24 & 108 & 64 & 45 & 2 & 43.4 & 0.4 & 28 & 2 & 43.4 & 0.8 \\
\hline 67 & 0.0505 & 0.0031 & 0.1400 & 0.0083 & 0.0201 & 03 & $0 . c$ & 0.0001 & 0.87 & 217 & 111 & 133 & 7 & 128 & 2 & 133 & 3 & 128 & 4 \\
\hline 068 & 0.0499 & 0.0032 & 0.1676 & 0.0106 & 0.0 & 0.0003 & 0.0 & 0.0002 & 1.20 & 190 & 121 & 157 & 9 & 155 & 2 & 169 & 4 & 155 & 4 \\
\hline $10 \mathrm{TC}$ & 0.0769 & 0.0013 & 2.0101 & 0.0306 & & 15 & 0.0596 & 0. & 1.18 & 1118 & 18 & 1119 & 10 & 1120 & 8 & 69 & 11 & 1118 & 36 \\
\hline 070 & .0769 & 0.0014 & 2.0259 & 0.0314 & 0.1912 & 0.0015 & 0.0638 & 0.0008 & 1.9 & 1118 & 19 & 1124 & 11 & 1128 & 8 & 1250 & 15 & 1118 & 38 \\
\hline 071 & 0.0501 & 0.0106 & 0.0690 & 0.0144 & 0.0100 & 0.0004 & 0.0032 & 0.0002 & 1.23 & 200 & 334 & 68 & 14 & 64 & 2 & 64 & 5 & 64 & 4 \\
\hline 072 & .0581 & 0.0008 & 0.6114 & 0.0068 & 0.0763 & 0.0005 & 0.0226 & 0.0002 & 2.03 & 534 & 13 & 484 & 4 & 474 & 3 & 452 & 4 & 474 & 6 \\
\hline 073 & .0635 & 0.0051 & 0.8012 & 0.0639 & & 0.0014 & 0.0285 & 0005 & 0.34 & 724 & 145 & 598 & 36 & 565 & 8 & 567 & 9 & 565 & 16 \\
\hline 074 & 0.0490 & 0.0015 & 0.0743 & 0.0022 & 0.0110 & 0.0001 & 0.0037 & 0.0001 & 1.30 & 147 & 53 & 73 & 2 & 70.5 & 0.6 & 75 & 1 & 70.5 & 1.2 \\
\hline OTQ06-075 & 0.0533 & 0.0011 & 0.3941 & 0.0070 & 0.0537 & 0.0004 & 0.0172 & 0.0004 & 10.56 & 340 & 27 & 337 & 5 & 337 & 2 & 344 & 9 & 337 & 4 \\
\hline
\end{tabular}




\begin{tabular}{|c|c|c|c|c|c|c|c|c|c|c|c|c|c|c|c|c|c|c|c|}
\hline 0TQ06-076 & .0528 & 0.0033 & 0.1209 & 0.0073 & 0.0166 & 0.0002 & 0.0061 & 0.0001 & 0.99 & 322 & 112 & 116 & 7 & 106 & 2 & 123 & 3 & 106 & 4 \\
\hline 0TQ06-077 & .0738 & 0.0011 & 1.7604 & 0.0221 & 1730 & 0.0013 & 0.0546 & 0.0006 & 2.12 & 1036 & 14 & 1031 & 8 & 1029 & 7 & 1074 & 11 & 1036 & 28 \\
\hline 0TQ06-078 & 0501 & 0.0108 & 0.1219 & 0.0261 & .0176 & 0.0005 & 0.0075 & 0.0005 & 1.35 & 201 & 351 & 117 & 24 & 113 & 3 & 152 & 10 & 113 & 6 \\
\hline OTQ06-079 & 0476 & 0.0060 & 0.0509 & .0063 & .0078 & 0.0002 & 0.0027 & .0001 & 0.84 & 78 & 227 & 50 & 6 & 50 & 1 & 54 & 2 & 50 & 2 \\
\hline TTQ06-080 & .0482 & 0.0027 & 0.0694 & .0038 & .0105 & 0.0001 & 0.0037 & 0.0001 & 0.97 & 107 & 100 & 68 & 4 & 67.1 & 0.8 & 74 & 1 & 67.1 & 1.6 \\
\hline JTQ06-081 & .0506 & 0.0034 & 0.1072 & 0070 & 0154 & 0.0002 & 0.0047 & 0.0002 & 1.27 & 221 & 120 & 103 & 6 & 98 & 0 & 94 & 3 & 98 & 4 \\
\hline TTQ06-082 & .0527 & .0026 & 0.2896 & 0137 & 0399 & 0.0005 & 0.0148 & 0.0004 & 1.92 & 317 & 85 & 258 & 11 & 252 & & 298 & 8 & 252 & 6 \\
\hline OTQ06-083 & .0493 & .0185 & 0.0607 & .0228 & .0089 & 0.0002 & 0.0014 & .0004 & 1.19 & 160 & 600 & 60 & 22 & 57 & & 29 & 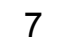 & 57 & 2 \\
\hline DTC & 0668 & .00 & 32 & 207 & 2 & 9 & 0.0 & 99 & 3.99 & 83 & 32 & 659 & 11 & 610 & & 871 & 17 & 610 & 10 \\
\hline DTC & .0469 & 0041 & 40 & 63 & 15 & 0.0002 & 07 & 1 & 1.25 & 45 & 162 & 73 & & 73 & & 13 & & 73 & 2 \\
\hline 36 & 0645 & 0012 & 9291 & 0156 & 1046 & 08 & 18 & 33 & 1.67 & 75 & 2 & 66 & & 64 & & 6 & & 41 & 10 \\
\hline 37 & 0754 & 10 & 1.8679 & 0187 & 99 & 12 & 76 & 04 & 1.3 & 10 & 10 & 1070 & & 1066 & & 132 & 8 & 078 & 20 \\
\hline 88 & 464 & 0013 & 0.0375 & .0010 & 0059 & 00 & 18 & 00 & 0.67 & 18 & 45 & 37 & & 37.7 & 0.3 & 35.7 & 0.4 & 37.7 & 0.6 \\
\hline 89 & 1499 & .0021 & 8.4278 & 1014 & 4077 & .0031 & 0.1143 & 0008 & 1.69 & 2345 & 2 & 2278 & 11 & 2204 & 14 & 2187 & 15 & 2345 & 50 \\
\hline 90 & 0461 & .0066 & 0.1327 & 0.0189 & .0209 & .0004 & 0.0067 & .0003 & 0.88 & & 262 & 12 & 17 & 13 & & 135 & 0 & 133 & 6 \\
\hline 091 & 0671 & 0.0019 & 1.1076 & .0298 & 1197 & .0012 & 0.0403 & 06 & 1.04 & 842 & 39 & 757 & 14 & 729 & & 798 & 11 & 729 & 14 \\
\hline $6-092$ & 1060 & 0013 & 4.0968 & 0405 & 2803 & 0.0020 & 0.0617 & 0011 & 5.54 & 1732 & 9 & 1654 & 0 & 1593 & 0 & 1209 & 20 & 1732 & 18 \\
\hline 0TQ06-093 & .0484 & 0.0024 & 0.0472 & 0.0023 & 0.0071 & 0.0001 & 0.0023 & .0000 & 1.07 & 11 & 89 & 47 & 2 & 45.4 & 0.5 & 46.8 & 0.8 & 45.4 & 1 \\
\hline $6-094$ & .0489 & 0.0252 & 0.0246 & 0.0127 & 0.0037 & 0.0002 & 0.0013 & 0.0001 & 0.67 & 14 & 833 & 25 & 13 & 23 & 1 & 26 & 2 & 23 & 2 \\
\hline $6-095$ & 0894 & 0.0013 & 3.0078 & .0371 & 2442 & .0018 & 0.0750 & .0007 & 1.31 & 1412 & 13 & 1410 & 9 & 1409 & 9 & 1463 & 13 & 1412 & 26 \\
\hline 10TQ06-096 & 0.0473 & 0.0087 & 0.0714 & 0.0130 & 0.0110 & 0.0002 & 0.0028 & 0.0002 & 1.03 & 62 & 301 & 70 & 12 & 70 & 1 & 57 & 4 & 70 & 2 \\
\hline 10TQ06-097 & 0.0584 & 0.0046 & 0.7361 & 0.0570 & 0.0914 & 0.0017 & 0.0342 & 0.0028 & 5.42 & 545 & 139 & 560 & 33 & 564 & 10 & 679 & 55 & 564 & 20 \\
\hline 10TQ06-098 & 0.0461 & 0.0055 & 0.0904 & 0.0106 & .0142 & 0.0003 & 0.0047 & 0.0002 & 0.99 & & 237 & 88 & 10 & 91 & 2 & 95 & 5 & 91 & 4 \\
\hline 10TQ06-099 & 0.0786 & 0.0012 & 2.1375 & 0.0295 & 0.1973 & 0.0015 & 0.0591 & 0.0008 & 38.49 & 1162 & 32 & 1161 & 10 & 1161 & 8 & 1161 & 14 & 1162 & 64 \\
\hline 10TQ06-100 & 0.1641 & 0.0022 & 10.5921 & 0.1160 & .4683 & 0.0038 & 0.1430 & 0.0012 & 0.71 & 2498 & 9 & 2488 & 10 & 2476 & 17 & 2702 & 21 & 2498 & 18 \\
\hline 10TQ06-101 & 0.0692 & 0.0010 & 1.4398 & .0166 & 0.1509 & 0.0011 & 0.0489 & 0.0004 & 1.99 & 905 & 13 & 906 & 7 & 906 & 6 & 964 & 8 & 906 & 12 \\
\hline 10TQ06-102 & 0.1161 & 0.0013 & 5.1602 & 0.0452 & 0.3224 & 0.0021 & 0.0927 & 0.0007 & 8.25 & 1896 & 20 & 1846 & 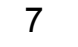 & 1802 & 10 & 1793 & 13 & 1896 & 40 \\
\hline 10TQ06-103 & 0.0501 & 0.0055 & 0.1398 & .0151 & .0203 & 0.0003 & 0.0006 & 0.0003 & 1.58 & 199 & 222 & 133 & 13 & 129 & 2 & -13 & 6 & 129 & 4 \\
\hline 10TQ06-104 & 0.0498 & 0.0046 & 0.0503 & 0.0046 & 0.0073 & 0.0001 & 0.0027 & 0.0001 & 0.98 & 184 & 181 & 50 & 4 & 47.1 & 0.6 & 53 & 1 & 47.1 & 1.2 \\
\hline DTQ06-105 & 0480 & 0091 & 0621 & 0117 & 094 & 0002 & 0032 & 0002 & 1.08 & 99 & 307 & 61 & 11 & 60 & 1 & 64 & 3 & 60 & 2 \\
\hline
\end{tabular}

$2010 \mathrm{TQ16}$ (Qiuwu, Qiabulin section, $\mathrm{n}=103, \mathrm{~N}^{\circ} 9^{\circ} 19.892^{\prime} \mathrm{E88^{ \circ }} 30.925^{\prime}, 4042 \mathrm{~m}$ )

$\begin{array}{llllllllllllllllllll}\text { 10TQ16-002 } & 0.0593 & 0.0011 & 0.7495 & 0.0134 & 0.0918 & 0.0008 & 0.0257 & 0.0003 & 1.68 & 577 & 23 & 568 & 8 & 566 & 5 & 514 & 6 & 566 & 10\end{array}$ $\begin{array}{llllllllllllllllllll}\text { 10TQ16-003 } & 0.1106 & 0.0011 & 5.2683 & 0.0441 & 0.3457 & 0.0028 & 0.0980 & 0.0006 & 1.38 & 1809 & 7 & 1864 & 7 & 1914 & 13 & 1890 & 11 & 1809 & 14\end{array}$ 


\begin{tabular}{|c|c|c|c|c|c|c|c|c|c|c|c|c|c|c|c|c|c|c|c|}
\hline 0TQ16-004 & .0516 & 0.0013 & 0.2771 & 0.0065 & 0.0390 & 0.0004 & 0.0119 & 0.0001 & 0.82 & 267 & 37 & 248 & - & 246 & 2 & 239 & 2 & 246 & 4 \\
\hline 10TQ16-005 & 0.0500 & 0.0070 & 0.1262 & 0.0176 & 0.0183 & 0.0004 & 0.0067 & 0.0003 & 1.75 & 196 & 266 & 121 & 16 & 117 & 2 & 136 & 6 & 117 & 1 \\
\hline 0TQ16-006 & 0.0482 & 0.0047 & 0.1045 & 0.0101 & 0.0157 & 0.0002 & 0.0083 & 0.0006 & 5.51 & 111 & 191 & 101 & 0 & 100 & 1 & 167 & 11 & 00 & 2 \\
\hline 0TQ16-007 & 0.0521 & 0.0100 & 0.0556 & 0.0106 & 0.0077 & 0.0002 & 0.0024 & 0.0002 & 2.44 & 289 & 370 & 55 & 10 & 49.7 & 1 & 49 & 5 & 49.7 & \\
\hline 0TQ16-008 & 0.1102 & 0.0013 & 5.3992 & 0.0535 & 0.3554 & 0.0029 & 0.1267 & 0.0014 & 5.86 & 1803 & 8 & 1885 & 0 & 1961 & 14 & 2410 & 25 & 1803 & 6 \\
\hline JTQ16-009 & 0.0644 & $O C$ & 1.0492 & 0.0210 & 0.1182 & 0.0011 & 0.0369 & 5 & 2.19 & 754 & 27 & 728 & 10 & 720 & 6 & 733 & 0 & 720 & 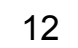 \\
\hline-010 & 0.1 & 0016 & 4.6980 & 0.0658 & 3246 & .0030 & 0.0926 & 0012 & .25 & 715 & 13 & 767 & 12 & 812 & 14 & 790 & 3 & 15 & \\
\hline TQ16. & 0.0511 & 0009 & .2530 & 0.0038 & 0360 & .0003 & 0.0119 & 02 & 06 & 243 & 20 & 229 & & 228 & 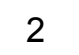 & 239 & & 228 & \\
\hline 012 & 24 & 15 & .5032 & 0.0659 & 11 & 32 & 35 & 07 & 11 & 2130 & & 2173 & 8 & 2220 & 15 & 172 & 3 & 130 & \\
\hline-013 & 62 & 0014 & .7774 & 0.0560 & 3265 & 0028 & 0.0953 & 08 & 1.03 & 735 & 10 & 781 & 10 & 1821 & 13 & 840 & 5 & 735 & \\
\hline TTQ16-014 & 0.0777 & 0015 & 2.1157 & 0.0376 & 1975 & .0017 & 0.0593 & 005 & 2.06 & 1139 & 40 & 154 & 12 & 1162 & 9 & 163 & & 139 & \\
\hline TTQ16-015 & 0.0682 & .0033 & 0.9666 & 0.0463 & 1029 & 0.0013 & 0.0316 & 0.0004 & 0.32 & 873 & $7 \varepsilon$ & 687 & 24 & 631 & 8 & 529 & & 631 & \\
\hline TTQ16-016 & 0.0957 & .0014 & 3.5694 & 0.0442 & .2706 & 0.0023 & 0.0771 & 0.0008 & 2.02 & 1542 & 12 & 543 & 10 & 1544 & 1 & 501 & 15 & 542 & \\
\hline 0TQ16-017 & 0.1925 & 0.0022 & 14.7950 & 0.1360 & .5577 & 0.0045 & 0.1557 & 0.0013 & 1.30 & 2763 & 7 & 2802 & 0 & 2857 & 19 & 2925 & 22 & 2763 & \\
\hline JTQ16-018 & 0.0501 & 0.0017 & 0.1012 & 0.0033 & 0.0147 & 0.0002 & 0.0048 & 0.0001 & 0.99 & 201 & 57 & 98 & 3 & 93.8 & 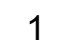 & 96 & 1 & 93.8 & \\
\hline -019 & 0.0935 & 0.0013 & 3.3658 & 0.0403 & .2613 & 0.0021 & 0.0776 & 0.0007 & 1.13 & 1497 & 1 & 1496 & 9 & 1496 & 11 & 1511 & 12 & 1497 & 2 \\
\hline 020 & 0.0553 & .00 & 0.2757 & 0.0072 & 0361 & 0.0003 & 0.0122 & 01 & 0.8 & 426 & 42 & 247 & 6 & 229 & 0 & 245 & 3 & 229 & \\
\hline 10TQ & 0.0820 & 0.0024 & 2.2399 & 0.0623 & 0.1982 & 0.0023 & 0.0601 & 0.0009 & 0.86 & 1245 & 36 & 1194 & 20 & 1166 & 12 & 1179 & 18 & 1245 & 12 \\
\hline-022 & 0.0598 & 0.0011 & 0.7703 & 0.0131 & 0.0935 & 0.0008 & 0.0301 & 0.0003 & 0.88 & 595 & 23 & 580 & 8 & 576 & 5 & 598 & 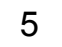 & 576 & 10 \\
\hline 5-023 & 0.0461 & 0.0016 & 0.0877 & 0.0028 & 0.0138 & 0.0002 & 0.0048 & 0.0003 & 1.03 & & 74 & 85 & 3 & 88 & 1 & 97 & 5 & 88 & $\angle$ \\
\hline 0TQ16-024 & 0.0612 & 0.0016 & 0.7535 & 0.0183 & 0.0893 & 0.0008 & 0.0300 & 0.0005 & 1.84 & 647 & 36 & 570 & 11 & 551 & 5 & 598 & 9 & 551 & 10 \\
\hline 25 & 0.1 & 4 & & 0.1454 & 58 & 0.0 & 0.0 & 07 & 0.13 & 1886 & 102 & 1061 & 52 & 706 & 19 & 903 & 14 & 706 & 38 \\
\hline OTC & 0.0 & .00 & & 0.0607 & & 0.0 & 0.0 & & 2.58 & 1118 & 46 & 1054 & 22 & 1023 & 12 & 1259 & 33 & 1118 & 92 \\
\hline 10TC & 0.0 & 4 & & 0.0 & 38 & 0.0 & & & 1.57 & 236 & 42 & 126 & 3 & 120 & 1 & 125 & 2 & 120 & 2 \\
\hline 10TC & 0.0 & 8 & & 0.0 & & & & & 1.75 & 129 & 195 & 127 & 12 & 127 & 2 & 8 & 6 & 27 & 4 \\
\hline $10 \mathrm{TC}$ & 0. & 2 & & 0.0 & & & 02 & & 1.22 & 1488 & 9 & 1494 & 8 & 1498 & 10 & 1560 & 12 & 1488 & 8 \\
\hline 30 & 0 & 00 & 31 & 0.0112 & 0948 & 0.0007 & 0.0322 & 04 & 4.3 & 602 & $1 \varepsilon$ & 58 & 0 & 58 & 1 & 641 & 8 & 584 & 8 \\
\hline 0TQ16-031 & 0.0688 & 0013 & 1.4072 & 0.0242 & 1484 & 0.0012 & 0.0488 & 0.0006 & 2.11 & 892 & 22 & 89 & 10 & 89 & 7 & 964 & 12 & 892 & 4 \\
\hline OTQ16-032 & 0.0533 & 0033 & 2408 & 0.0148 & 28 & 0.0004 & 0.0060 & 0.0002 & 0.6 & 341 & 118 & 21 & 12 & 20 & & 122 & 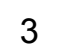 & 208 & \\
\hline 0TQ16-033 & 0.0636 & 0013 & 0.9699 & 0.0188 & 1106 & 0.0009 & 0.0349 & 0.0006 & 3.34 & 729 & 27 & 688 & 10 & 676 & 5 & 694 & 12 & 676 & 0 \\
\hline 0TQ16-034 & 0.0750 & 0011 & 1.8081 & 0.0236 & 1749 & 0.0013 & 0.0581 & 0.0007 & 2.77 & 1069 & 15 & 1048 & 9 & 1039 & 7 & 1141 & 13 & 1069 & 30 \\
\hline 0TQ16-035 & 0.0471 & 0068 & 0.0527 & 0.0075 & 0.0081 & 0.0002 & 0.0028 & 0.0001 & 0.97 & 56 & 247 & 52 & 7 & 52 & 1 & 57 & & 52 & 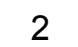 \\
\hline 0TQ16-036 & 0.0672 & 0015 & 1.2923 & 0.0270 & 0.1396 & 0.0011 & 26 & 0.0003 & 2.69 & 843 & 48 & 842 & 12 & 842 & 6 & 842 & 6 & 842 & 2 \\
\hline OTQ16-037 & 0.0504 & 0.0063 & 0.0582 & 0.0073 & 0.0084 & 0.0001 & 0.0036 & 0.0001 & 1.31 & 211 & 256 & 57 & 7 & 53.8 & 0.7 & 72 & 2 & 53.8 & \\
\hline
\end{tabular}




\begin{tabular}{|c|c|c|c|c|c|c|c|c|c|c|c|c|c|c|c|c|c|c|c|}
\hline 10TQ16-038 & .0490 & 0.0239 & 0.1442 & 0.0702 & 0.0214 & 0.0006 & 0.0106 & 0.0009 & 1.38 & 145 & 805 & 137 & 62 & 136 & 4 & 214 & 18 & 136 & 8 \\
\hline 0TQ16-039 & .0530 & 0.0032 & 0.3136 & 0.0187 & 0.0429 & 0.0005 & 0.0148 & 0.0004 & 1.42 & 328 & 115 & 277 & 14 & 271 & ? & 296 & 8 & 271 & 6 \\
\hline 0TQ16-040 & 0.0521 & 0.0080 & 0.1309 & 0.0200 & 0.0182 & 0.0004 & 0.0062 & 0.0003 & 0.83 & 291 & 298 & 125 & 18 & 116 & 0 & 126 & 5 & 116 & ; \\
\hline DTQ16-041 & 0.0585 & 0.0017 & 0.5674 & 0.0159 & 0.0704 & 0.0006 & 0.0229 & 0.0004 & 1.42 & 548 & 45 & 456 & 10 & 439 & 1 & 458 & 7 & 439 & 3 \\
\hline 0TQ16-042 & 0.0786 & 0.0024 & 1.9994 & 0.0579 & 0.1844 & 0.0017 & 0.0552 & 0.0004 & 1.28 & 1163 & 62 & 1115 & 20 & 1091 & 9 & 1087 & 8 & 163 & 4 \\
\hline JTQ16-043 & 0.0474 & 0.0030 & 0.0829 & 0.0051 & 0.0127 & 0.0001 & 0.0041 & 0.0001 & 0.95 & 69 & 114 & 81 & 5 & 81.3 & 0.9 & 82 & 2 & 31.3 & 0 \\
\hline DTQ16-044 & 0.0809 & .0016 & 2.2779 & 0.0416 & 0.2043 & 0.0018 & 0.0619 & 0.0006 & 0.58 & 1219 & 22 & 1205 & 13 & 1198 & 9 & 1215 & 10 & 1219 & 1 \\
\hline OTQ16-045 & 0.0476 & .0030 & 0.0291 & 0.0018 & 0.0044 & 0.0001 & 0.0014 & 0.0000 & 0.80 & 79 & 116 & 29 & 2 & 28.5 & 0.3 & 27.9 & 0.6 & 28.5 & 6 \\
\hline 46 & 0.05 & 0091 & 15 & 0.0166 & 2 & .0003 & 0.0 & 33 & 1.48 & 201 & 324 & 89 & 15 & 85 & ? & 70 & 6 & 85 & \\
\hline 47 & 0.02 & 43 & 35 & .0084 & 0146 & 3 & 42 & 01 & 0.53 & 155 & 158 & 9 & & 93 & & 85 & & 93 & \\
\hline 48 & 35 & 60 & 0 & .0167 & 04 & 03 & 34 & 02 & 0.73 & 170 & 237 & 13 & 15 & 130 & & 128 & & 130 & \\
\hline 049 & 98 & 0010 & 0.7264 & .0109 & 0881 & 06 & 76 & 03 & 2.09 & 596 & . & 554 & & 544 & & 50 & & 44 & \\
\hline 550 & 0.0537 & 0023 & 0.3098 & 0.0127 & 0419 & .0005 & 0.0119 & .0003 & 2.25 & 35 & 73 & 274 & 10 & 265 & & 40 & & 65 & \\
\hline 051 & 0.0687 & .0010 & 1.3915 & 0.0182 & 1469 & 0.0010 & 0.0447 & 0.0003 & 3.60 & 890 & 31 & 885 & & 883 & & 883 & 5 & 883 & 2 \\
\hline 052 & 0.1237 & .0018 & 5.6520 & 0.0699 & 3316 & .0027 & 0.0920 & .0010 & 1.30 & 2010 & 1 & 1924 & 11 & 1846 & 13 & 778 & 18 & 010 & 2 \\
\hline OTQ16-053 & 0.0614 & 0.0010 & 0.8456 & 0.0114 & 0.0999 & .0007 & 0.0314 & 0.0003 & 2.82 & 653 & 17 & 622 & 0 & 614 & 4 & 625 & 7 & 614 & \\
\hline 054 & 0.0593 & .0014 & 0.7471 & 0.0169 & 0.0915 & 0.0008 & 0.0281 & 0.0003 & 0.81 & 577 & 35 & 567 & 10 & 564 & 4 & 559 & 6 & 564 & \\
\hline 055 & 0.0803 & 0.0024 & 2.2319 & 0.0639 & 0.2017 & 0.0022 & 0.0610 & 0.0006 & 0.28 & 1204 & 39 & 1191 & 20 & 1184 & 12 & 1197 & 11 & 1204 & 78 \\
\hline 56 & 0.0483 & 0.0038 & 0.0943 & 0.0074 & 0.0142 & 0.0002 & 0.0047 & 0.0001 & 1.02 & 113 & 146 & 91 & 7 & 9 & 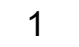 & 94 & 3 & 91 & \\
\hline 10TQ16-057 & 0.0605 & 0.0009 & 0.8167 & 0.0097 & 0.0979 & 0.0007 & 0.0321 & 0.0003 & 3.19 & 623 & 14 & 606 & $\Gamma$ & 602 & 4 & 638 & 6 & 602 & \\
\hline 10TQ16-058 & 0.2455 & 0.0027 & 9.0520 & 0.1501 & 0.5631 & 0.0040 & 0.1441 & 0.0010 & 1.11 & 3156 & 6 & 3044 & 8 & 2879 & 16 & 2721 & 18 & 3156 & 12 \\
\hline $10 \mathrm{TC}$ & 0.0560 & 0.0033 & 0.2938 & 0.0169 & 0.0381 & 0.0004 & 0.0104 & 0.0003 & 0.89 & 452 & 110 & 262 & 13 & 241 & ? & 209 & 5 & 241 & 4 \\
\hline 060 & 0.0810 & 0.0012 & 2.3117 & 0.0293 & 0.2071 & 0.0015 & 0.0633 & 0.0005 & 1.10 & 1221 & 14 & 1216 & 9 & 1213 & 8 & 1240 & 10 & 1221 & 28 \\
\hline 61 & 0.0840 & 0.0024 & & 0.0641 & 0.2037 & 0.0022 & 0.0634 & 0.0008 & 0.60 & 1292 & 36 & 1230 & 19 & 1195 & 12 & 1242 & 15 & 1292 & 12 \\
\hline 10TC & 0.0721 & 0.0024 & & 0.0426 & & 15 & & & 0.49 & 988 & 46 & 868 & 18 & 823 & 0 & 846 & 10 & 823 & 8 \\
\hline 10TC & 0.0 & 0.0012 & & 0.0182 & & & & & 1.07 & 904 & 19 & 807 & 0 & 772 & 5 & 95 & 6 & 72 & U \\
\hline 10TC & 0.0 & 0.0 & & 0.0224 & & & & & 5.68 & 1084 & 28 & 088 & 8 & 1090 & 7 & 1091 & 7 & 1084 & 6 \\
\hline 10TC & & & & & & & & & 1.07 & 589 & 1 & 612 & 6 & 619 & 4 & 0 & 5 & 9 & 8 \\
\hline 66 & 0.0673 & 14 & & 0.0251 & 0.1390 & 11 & & 03 & 2.5 & 848 & 44 & 84 & 11 & 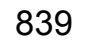 & & 839 & 6 & 839 & 2 \\
\hline 067 & 0.0730 & 0009 & & 0.0167 & 1705 & 0.0012 & 0.0504 & 0.0004 & 2.32 & 1015 & 10 & 1014 & 6 & 1015 & 0 & 994 & 8 & 1015 & 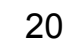 \\
\hline 068 & 0.1122 & 0012 & 5.2144 & 0.0386 & 3371 & 0.0022 & 0.0912 & 0.0007 & 3.50 & 1836 & 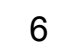 & 1855 & 6 & 1873 & 11 & 1764 & 12 & 1836 & 5 \\
\hline & & & & & & 019 & & 05 & 0.80 & 1589 & 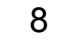 & 1589 & 7 & 1589 & 10 & 1611 & 9 & 1589 & 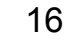 \\
\hline & 0.0494 & 0014 & & 0.0012 & & 0.0001 & 0.0021 & 0.0000 & 0.49 & 166 & 45 & 46 & 1 & 43.4 & 0.4 & 41.8 & 0.4 & 43.4 & .8 \\
\hline 10TQ16-071 & 0.0661 & 0.0009 & 1.1523 & 0.0135 & 0.1264 & 0.0008 & 0.0386 & 0.0002 & 5.62 & 810 & 29 & 778 & 6 & 767 & 5 & 765 & 5 & 767 & 0 \\
\hline
\end{tabular}




\begin{tabular}{|c|c|c|c|c|c|c|c|c|c|c|c|c|c|c|c|c|c|c|c|}
\hline 10TQ16-072 & 0.0675 & 0.0010 & 0.9323 & 0.0123 & 0.1002 & 0.0007 & 0.0255 & 0.0002 & 1.57 & 853 & 16 & 669 & 6 & 616 & 4 & 509 & 5 & 616 & 8 \\
\hline 10TQ16-073 & 0.0523 & 0.0027 & 0.1814 & 0.0090 & 0.0252 & 0.0003 & 0.0075 & 0.0001 & 0.63 & 300 & 91 & 169 & 8 & 160 & 2 & 151 & 2 & 160 & 4 \\
\hline 10TQ16-074 & 0.0520 & 0.0045 & 0.1021 & 0.0087 & 0.0143 & 0.0002 & 0.0065 & 0.0003 & 4.45 & 285 & 176 & 99 & 8 & 91.2 & 1 & 131 & 6 & 1.2 & 2 \\
\hline 10TQ16-075 & .1084 & 0.0013 & 4.8222 & .0430 & .3229 & 0.0022 & 0.0960 & 0.0012 & 6.38 & 1772 & 8 & 1789 & 7 & 1804 & 11 & 1853 & 22 & 1772 & 16 \\
\hline 10TQ16-076 & 0.0482 & 0.0043 & 0.0929 & .0081 & .0140 & .0002 & .0046 & 0.0001 & 1.02 & 108 & 164 & 90 & 8 & 90 & 1 & 93 & 3 & 90 & 2 \\
\hline 10TQ16-077 & 0.0600 & 0.0007 & 0.7530 & 0.0071 & 0.0911 & 0.0006 & 0.0260 & 0.0002 & 1.74 & 602 & 10 & 570 & 4 & 562 & 4 & 519 & 3 & 562 & 8 \\
\hline 10TQ16-078 & 0.0733 & 0.0012 & 1.5312 & 0.0230 & 0.1514 & 0.0011 & 0.0457 & 0.0003 & 2.23 & 1023 & 34 & 943 & 9 & 909 & 6 & 903 & 6 & 909 & 12 \\
\hline 10TQ16-079 & 0.0608 & 0.0009 & 0.8750 & .0102 & .1045 & .0007 & .0323 & 0.0002 & 1.39 & 630 & 14 & 638 & 6 & 641 & 4 & 642 & 5 & 641 & 8 \\
\hline 10TQ16-080 & 0.1834 & 0.0022 & 12.6024 & 0.1162 & 0.4986 & 0.0037 & .1240 & 0.0012 & 1.77 & 2684 & 7 & 2650 & 9 & 2608 & 16 & 2363 & 22 & 2684 & 14 \\
\hline 10TQ16-081 & 0.0622 & 0.0009 & 1.0004 & 0.0125 & 0.1166 & 0.0008 & 0.0369 & 0.0005 & 5.40 & 682 & 15 & 704 & 6 & 711 & 5 & 731 & 10 & 711 & 10 \\
\hline OTQ16-082 & 0.0608 & 0.0008 & 0.7894 & .0075 & .0941 & .0006 & .0229 & 0.0002 & .08 & 633 & 10 & 591 & 4 & 580 & 4 & 457 & 5 & 580 & 8 \\
\hline 0TQ16-083 & 0.0560 & 0.0009 & 0.5575 & 0.0076 & 0.0722 & 0.0005 & .0237 & 0.0002 & 2.21 & 453 & 18 & 450 & 5 & 449 & 3 & 473 & 4 & 449 & 6 \\
\hline 10TQ16-084 & 0.0516 & 0.0031 & 0.1040 & 0.0060 & 0.0146 & 0.0002 & 0.0048 & 0.0001 & 1.17 & 267 & 110 & 100 & 6 & 94 & 1 & 98 & 2 & 94 & 2 \\
\hline 10TQ16-085 & 0.0475 & 0.0071 & 0.1105 & 0.0163 & 0.0169 & 0.0004 & .0053 & 0.0003 & 1.53 & 75 & 258 & 106 & 15 & 108 & 2 & 106 & 6 & 108 & 4 \\
\hline 10TQ16-086 & 0.0755 & 0.0012 & 1.9126 & 0.0274 & 0.1838 & 0.0014 & .0557 & 0.0005 & 1.23 & 1082 & 17 & 1085 & 10 & 1088 & 8 & 095 & 0 & 1082 & 4 \\
\hline 10TQ16-087 & 0.0806 & 0.0015 & 2.2419 & 0.0388 & 0.2017 & 0.0017 & 0.0586 & 0.0005 & 0.49 & 1213 & 21 & 1194 & 12 & 1184 & 9 & 1151 & 9 & 1213 & 42 \\
\hline 10TQ16-088 & 0.0560 & 0.0008 & 0.5362 & 0.0068 & 0.0694 & 0.0005 & .0196 & 0.0002 & 1.99 & 454 & 16 & 436 & 5 & 433 & 3 & 393 & 4 & 433 & 6 \\
\hline 10TQ16-089 & 0.0622 & 0.0010 & 0.9011 & 0.0124 & 0.1051 & 0.0008 & 0.0308 & 0.0005 & 5.67 & 681 & 17 & 652 & 7 & 644 & 4 & 614 & 10 & 644 & 8 \\
\hline 0TQ16-090 & 0.0477 & 0.0048 & 0.0539 & 0.0053 & 0.0082 & 0.0001 & 0.0026 & 0.0001 & 0.86 & 82 & 193 & 53 & 5 & 52.6 & 0.8 & 52 & 1 & 52.6 & 1.6 \\
\hline 10TQ16-091 & 0.2476 & 0.0027 & 21.5474 & 0.1740 & 0.6315 & 0.0046 & 0.1574 & 0.0015 & 2.15 & 3169 & 6 & 3164 & 8 & 3155 & 18 & 2954 & 26 & 3169 & 12 \\
\hline 10TQ16-092 & 0.0516 & 0.0009 & 0.3 & 0.0051 & 0.0445 & 0.0003 & 0.0 & 0.0001 & 1.84 & 268 & 24 & 279 & 4 & 280 & 2 & 271 & 3 & 280 & 4 \\
\hline 0TQ16-093 & 0.1663 & 0.0018 & 11.3173 & 0.0868 & 0.4937 & 0.0034 & 0.1297 & 0.0008 & 0.90 & 2521 & 6 & 2550 & 7 & 2586 & 15 & 2464 & 14 & 2521 & 12 \\
\hline 10TQ16-094 & 0.0788 & 0.0010 & 2.2384 & 0.0217 & 0.2062 & 0.0014 & 0.0590 & 0.0005 & 2.04 & 1166 & 9 & 1193 & 7 & 1209 & 8 & 1158 & 9 & 1166 & 18 \\
\hline 10TQ16-095 & 0.0614 & 0.0010 & 0.9891 & 0.0131 & 0.1168 & 0.0008 & 0.0354 & 0.0004 & 2.92 & 655 & 17 & 698 & 7 & 712 & 5 & 703 & 8 & 712 & 10 \\
\hline 10TQ16-096 & 0.0521 & 0.0032 & 0.1037 & 0.0062 & 0.0145 & 0.0002 & 0.0047 & 0.0001 & 0.92 & 288 & 115 & 100 & 6 & 92 & 1 & 94 & 2 & 92 & 2 \\
\hline 10TQ16-097 & 0.0523 & 0.0030 & 0.0959 & 0.0054 & 0.0133 & 0.0002 & 0.0042 & 0.0002 & 2.38 & 299 & 106 & 93 & 5 & 85 & 1 & 84 & 3 & 85 & 2 \\
\hline 10TQ16-098 & 0.0794 & 0.0013 & 2.2677 & 0.0332 & 0.2072 & 0.0016 & 0.0581 & 0.0005 & 0.67 & 1183 & 17 & 1202 & 10 & 1214 & 9 & 1141 & 9 & 1183 & 34 \\
\hline 10TQ16-099 & 0.0571 & 0.0019 & 0.6961 & 0.0217 & 0.0884 & 0.0009 & 0.0252 & 0.0004 & 0.95 & 496 & 51 & 536 & 13 & 546 & 5 & 504 & 7 & 546 & 10 \\
\hline 10TQ16-100 & 0.0710 & 0.0011 & 1.5745 & 0.0209 & 0.1609 & 0.0012 & 0.0467 & 0.0004 & 1.20 & 957 & 15 & 960 & 8 & 962 & 7 & 922 & 8 & 962 & 14 \\
\hline 10TQ16-101 & 0.0703 & 0.0009 & 1.5319 & 0.0157 & 0.1581 & 0.0011 & 0.0482 & 0.0006 & 6.03 & 937 & 11 & 943 & 6 & 946 & 6 & 951 & 11 & 946 & 12 \\
\hline 10TQ16-102 & 0.0925 & 0.0019 & 3.2415 & 0.0598 & 0.2540 & 0.0021 & 0.0748 & 0.0006 & 1.83 & 1479 & 39 & 1467 & 14 & 1459 & 11 & 1458 & 10 & 1479 & 78 \\
\hline 10TQ16-103 & 0.0491 & 0.0104 & 0.0621 & 0.0131 & 0.0092 & 0.0002 & 0.0029 & 0.0002 & 0.87 & 153 & 344 & 61 & 12 & 59 & 2 & 58 & 3 & 59 & 4 \\
\hline 10TQ16-104 & 0.1855 & 0.0023 & 14.2447 & 0.1414 & 0.5572 & 0.0044 & 0.1482 & 0.0016 & 1.69 & 2702 & 8 & 2766 & 9 & 2855 & 18 & 2792 & 29 & 2702 & 16 \\
\hline
\end{tabular}


2010 TQ15 (Dazuqu, Qiabulin section, $\mathrm{n}=64, \mathrm{~N}^{\circ} 9^{\circ} 19.892^{\prime}$, E88 ${ }^{\circ} 30.925^{\prime}, 4042 \mathrm{~m}$ )

$\begin{array}{llllllllll}10 T Q 15-03 & 0.0544 & 0.0011 & 0.4171 & 0.0076 & 0.0556 & 0.0004 & 0.0174 & 0.0001\end{array}$

$\begin{array}{llllllllllll}10 \text { Q15-05 } & 0.0972 & 0.0012 & 3.6961 & 0.0319 & 0.2759 & 0.0018 & 0.0823 & 0.0009 & 6.46\end{array}$

$\begin{array}{lllllllllll}\text { 10TQ15-06 } & 0.0492 & 0.0034 & 0.0338 & 0.0023 & 0.0050 & 0.0001 & 0.0017 & 0.0001 & 2.85\end{array}$

$\begin{array}{lllllllllll}\text { 10TQ15-07 } & 0.0735 & 0.0010 & 1.6024 & 0.0169 & 0.1583 & 0.0010 & 0.0469 & 0.0006 & 6.34\end{array}$

$\begin{array}{lllllllllll}10 T Q 15-08 & 0.0583 & 0.0009 & 0.6102 & 0.0081 & 0.0760 & 0.0005 & 0.0221 & 0.0002 & 1.13\end{array}$

$\begin{array}{llllllllllll}\text { 10TQ15-09 } & 0.1269 & 0.0018 & 6.5532 & 0.0741 & 0.3747 & 0.0028 & 0.0981 & 0.0009 & 1.05\end{array}$

$\begin{array}{lllllllllll}\text { 10TQ15-10 } & 0.0486 & 0.0088 & 0.0893 & 0.0159 & 0.0133 & 0.0005 & 0.0047 & 0.0003 & 1.06\end{array}$

$\begin{array}{lllllllllll}\text { 10TQ15-11 } & 0.0483 & 0.0136 & 0.0810 & 0.0226 & 0.0122 & 0.0004 & 0.0042 & 0.0003 & 1.27\end{array}$

$\begin{array}{lllllllllll}\text { 10TQ15-12 } & 0.0507 & 0.0018 & 0.0911 & 0.0030 & 0.0130 & 0.0001 & 0.0039 & 0.0000 & 0.75\end{array}$

$\begin{array}{llllllllll}10 T Q 15-13 & 0.0498 & 0.0038 & 0.0893 & 0.0066 & 0.0130 & 0.0002 & 0.0036 & 0.0001 & 0.88\end{array}$

$\begin{array}{lllllllllll}\text { 10TQ15-14 } & 0.0487 & 0.0094 & 0.0637 & 0.0121 & 0.0095 & 0.0003 & 0.0032 & 0.0002 & 0.96\end{array}$

$\begin{array}{lllllllllll}\text { 10TQ15-15 } & 0.0582 & 0.0008 & 0.6558 & 0.0076 & 0.0818 & 0.0005 & 0.0244 & 0.0002 & 1.35\end{array}$

$\begin{array}{lllllllllll}\text { 10TQ15-16 } & 0.0490 & 0.0105 & 0.0524 & 0.0112 & 0.0078 & 0.0002 & 0.0024 & 0.0001 & 0.74\end{array}$

$\begin{array}{llllllllll}10 T Q 15-17 & 0.2388 & 0.0028 & 20.4096 & 0.1836 & 0.6200 & 0.0047 & 0.1508 & 0.0013 & 1.18\end{array}$

$\begin{array}{lllllllllll}\text { 10TQ15-18 } & 0.0480 & 0.0059 & 0.0874 & 0.0107 & 0.0132 & 0.0003 & 0.0040 & 0.0001 & 0.89\end{array}$

10TQ15-19

10TQ15-20

10TQ15-21

10TQ15-22

10TQ15-23

10TQ15-24

10TQ15-25

10TQ15-26

10TQ15-27

10TQ15-29

10TQ15-30

10TQ15-31

10TQ15-32

10TQ15-33

10TQ15-34

10TQ15-35

10TQ15-36

$0.0506 \quad 0.0015$

0.05320 .0063

$0.0473 \quad 0.0119$

$0.0502 \quad 0.0028$

$\begin{array}{ll}0.0496 & 0.0047\end{array}$

$0.0697 \quad 0.0013$

$\begin{array}{lllllll}0.1007 & 0.0029 & 0.0144 & 0.0001 & 0.0044 & 0.0001 & 1.41\end{array}$

$\begin{array}{llllllll}0.2031 & 0.0236 & 0.0277 & 0.0007 & 0.0096 & 0.0005 & 1.53\end{array}$

$\begin{array}{lllllll}0.0498 & 0.0124 & 0.0076 & 0.0003 & 0.0026 & 0.0002 & 1.27\end{array}$

$\begin{array}{llllllll}0.1867 & 0.0101 & 0.0270 & 0.0003 & 0.0077 & 0.0002 & 1.73\end{array}$

$\begin{array}{llllllll}0.1708 & 0.0160 & 0.0250 & 0.0004 & 0.0079 & 0.0002 & 1.59\end{array}$

$0.0591 \quad 0.0013$

$\begin{array}{lllllll}1.4868 & 0.0237 & 0.1547 & 0.0012 & 0.0433 & 0.0005 & 1.46\end{array}$

$\begin{array}{llllllll}0.7344 & 0.0145 & 0.0901 & 0.0007 & 0.0259 & 0.0004 & 3.11\end{array}$

$\begin{array}{llllllllll}0.3302 & 0.0036 & 34.2864 & 0.2587 & 0.7533 & 0.0052 & 0.1901 & 0.0016 & 1.90\end{array}$

$0.0503 \quad 0.0143$

$\begin{array}{ll}0.0487 & 0.0187\end{array}$

$0.0473 \quad 0.0074$

$0.0510 \quad 0.0038$

$0.0583 \quad 0.0038$

$\begin{array}{lll}0.0621 & 0.0173 & 0.009\end{array}$

$\begin{array}{llll}0.0005 & 0.0028 & 0.0004 & 1.52\end{array}$

$\begin{array}{lllllll}0.0578 & 0.0220 & 0.0086 & 0.0005 & 0.0035 & 0.0003 & 0.83\end{array}$

$\begin{array}{lllllll}0.0516 & 0.0079 & 0.0079 & 0.0002 & 0.0025 & 0.0001 & 0.80\end{array}$

$\begin{array}{llllllll}0.0962 & 0.0070 & 0.0137 & 0.0002 & 0.0044 & 0.0001 & 1.06\end{array}$

$\begin{array}{lllllll}0.4965 & 0.0311 & 0.0618 & 0.0010 & 0.0211 & 0.0009 & 2.41\end{array}$

$\begin{array}{llllllllll}0.0598 & 0.0011 & 0.7234 & 0.0115 & 0.0878 & 0.0006 & 0.0275 & 0.0003 & 2.26\end{array}$

$\begin{array}{lllllllll}0.0650 & 0.0012 & 1.1075 & 0.0187 & 0.1237 & 0.0009 & 0.0380 & 0.0005 & 1.91\end{array}$

$\begin{array}{lllllllll}0.0485 & 0.0032 & 0.0834 & 0.0054 & 0.0125 & 0.0002 & 0.0040 & 0.0001 & 1.04\end{array}$

$0.0978 \quad 0.0013$

$\begin{array}{lll}3.6963 & 0.0380 & 0.2741\end{array}$

$\begin{array}{llll}0.0019 & 0.0786 & 0.0006 & 1.14\end{array}$

$0.0515 \quad 0.0068$

$\begin{array}{cccccccccc}389 & 45 & 354 & 5 & 349 & 2 & 348 & 2 & 349 & 4 \\ 1571 & 8 & 1571 & 7 & 1571 & 9 & 1599 & 17 & 1571 & 16 \\ 158 & 123 & 34 & 2 & 32.1 & 0.5 & 35 & 1 & 32.1 & 1 \\ 1027 & 11 & 971 & 7 & 947 & 6 & 926 & 11 & 947 & 12 \\ 539 & 17 & 484 & 5 & 472 & 3 & 442 & 3 & 472 & 6 \\ 2055 & 10 & 2053 & 10 & 2051 & 13 & 1892 & 16 & 2055 & 20 \\ 127 & 293 & 87 & 15 & 85 & 3 & 96 & 6 & 85 & 6 \\ 114 & 425 & 79 & 21 & 78 & 2 & 85 & 6 & 78 & 4 \\ 226 & 62 & 88 & 3 & 83.5 & 0.7 & 78.3 & 0.8 & 83.5 & 1.4 \\ 184 & 139 & 87 & 6 & 83 & 1 & 73 & 2 & 83 & 2 \\ 135 & 304 & 63 & 12 & 61 & 2 & 65 & 4 & 61 & 4 \\ 536 & 14 & 512 & 5 & 507 & 3 & 488 & 3 & 507 & 6 \\ 147 & 352 & 52 & 11 & 50 & 1 & 48 & 2 & 50 & 2 \\ 3112 & 6 & 3111 & 9 & 3110 & 19 & 2839 & 24 & 3112 & 12 \\ 99 & 232 & 85 & 10 & 85 & 2 & 81 & 3 & 85 & 4 \\ 224 & 50 & 97 & 3 & 92.3 & 0.8 & 89 & 1 & 92.3 & 1.6 \\ 336 & 216 & 188 & 20 & 176 & 4 & 193 & 10 & 176 & 8 \\ 65 & 362 & 49 & 12 & 49 & 2 & 52 & 5 & 49 & 4 \\ 206 & 102 & 174 & 9 & 171 & 2 & 154 & 4 & 171 & 4 \\ 174 & 216 & 160 & 14 & 159 & 2 & 159 & 4 & 159 & 4 \\ 920 & 21 & 925 & 10 & 927 & 6 & 857 & 9 & 927 & 12 \\ 572 & 29 & 559 & 9 & 556 & 4 & 516 & 8 & 556 & 8 \\ 3618 & 5 & 3618 & 7 & 3619 & 19 & 3517 & 28 & 3618 & 10 \\ 208 & 420 & 61 & 17 & 58 & 3 & 56 & 7 & 58 & 6 \\ 131 & 558 & 57 & 21 & 55 & 3 & 71 & 6 & 55 & 6 \\ 63 & 258 & 51 & 8 & 51 & 1 & 51 & 2 & 51 & 2 \\ 239 & 140 & 93 & 7 & 88 & 1 & 88 & 2 & 88 & 2 \\ 540 & 108 & 409 & 21 & 387 & 6 & 422 & 17 & 387 & 12 \\ 595 & 22 & 553 & 7 & 543 & 4 & 547 & 6 & 543 & 8 \\ 773 & 23 & 757 & 9 & 752 & 5 & 753 & 9 & 752 & 10 \\ 122 & 121 & 81 & 5 & 80 & 1 & 81 & 2 & 80 & 2 \\ 1583 & 10 & 1571 & 8 & 1562 & 10 & 1530 & 11 & 1583 & 20 \\ 264 & 238 & 58 & 7 & 54 & 1 & 64 & 4 & 54 & 2\end{array}$




\begin{tabular}{|c|c|c|c|c|c|c|c|c|c|c|c|c|c|c|c|c|c|c|c|}
\hline 10TQ15-38 & 0.0695 & 0.0017 & 1.3638 & 0.0316 & 0.1423 & 0.0013 & 0.0419 & 0.0004 & 0.44 & 915 & 33 & 874 & 14 & 857 & 7 & 830 & 7 & 857 & 14 \\
\hline 10TQ15-39 & 0.0534 & 0.0023 & 0.3068 & 0.0127 & 0.0417 & 0.0005 & 0.0136 & 0.0003 & 1.97 & 347 & 74 & 272 & 10 & 263 & 3 & 273 & 6 & 263 & 6 \\
\hline 10TQ15-40 & 0.0500 & 0.0010 & 0.0992 & 0.0017 & 0.0144 & 0.0001 & 0.0045 & 0.0000 & 1.07 & 193 & 28 & 96 & 2 & 92.2 & 0.6 & 90.8 & 0.8 & 92.2 & 1.2 \\
\hline 10TQ15-41 & 0.0506 & 0.0018 & 0.1091 & 0.0037 & 0.0156 & 0.0001 & 0.0050 & 0.0001 & 1.05 & 223 & 62 & 105 & 3 & 100 & 0.9 & 101 & 1 & 100 & 1.8 \\
\hline OTQ15-42 & .0670 & 0.0014 & 1.2620 & .0248 & .1367 & .0011 & .0404 & 0.0004 & 0.90 & 836 & 27 & 829 & 11 & 826 & 6 & 800 & 8 & 826 & 12 \\
\hline OTQ15-43 & 0.0796 & 0.0010 & 2.2198 & 0.0212 & 0.2023 & 0.0013 & 0.0594 & 0.0004 & 1.16 & 1188 & 9 & 1187 & 7 & 1187 & 7 & 1166 & 8 & 188 & 18 \\
\hline 10TQ15-44 & 0.0480 & 0.0044 & 0.0534 & 0.0048 & 0.0081 & 0.0001 & 0.0026 & 0.0001 & 1.44 & 99 & 168 & 53 & 5 & 51.8 & 0.9 & 53 & 2 & 51.8 & 1.8 \\
\hline 0TQ15-45 & .0482 & 0.0015 & 0.1020 & 0.0030 & .0154 & .0001 & .0049 & 0.0001 & 0.82 & 108 & 53 & 99 & 3 & 98.3 & 0.8 & 98 & 1 & 98.3 & 1.6 \\
\hline 0TQ15-46 & 0.0478 & 0.0047 & 0.0949 & 0.0092 & 0.0144 & 0.0003 & .0050 & 0.0002 & 1.24 & 91 & 182 & 92 & 9 & 92 & 2 & 101 & 4 & 92 & 4 \\
\hline 0TQ15-48 & 0.0501 & 0.0068 & 0.0545 & 0.0073 & 0.0079 & 0.0002 & 0.0029 & 0.0001 & 0.79 & 201 & 253 & 54 & 7 & 51 & 1 & 58 & 2 & 51 & 2 \\
\hline 0TQ15-49 & 0.1319 & 0.0015 & 7.0872 & 0.0593 & 0.3897 & .0026 & .1122 & 0.0009 & 1.65 & 2124 & 7 & 2122 & 7 & 2121 & 12 & 2148 & 16 & 124 & 14 \\
\hline 0TQ15-50 & 0.0631 & 0.0014 & 0.9416 & 0.0188 & 0.1082 & 0.0009 & .0338 & 0.0006 & 3.41 & 713 & 29 & 674 & 10 & 662 & 5 & 671 & 12 & 662 & 10 \\
\hline 10TQ15-51 & 0.0825 & 0.0016 & 2.2797 & 0.0407 & 0.2006 & 0.0017 & 0.0615 & 0.0006 & 0.71 & 1256 & 22 & 1206 & 13 & 1178 & 9 & 1207 & 11 & 1256 & 44 \\
\hline 0TQ15-52 & 0.0517 & 0.0030 & 0.2153 & 0.0120 & 0.0302 & 0.0004 & 0.0115 & 0.0003 & 1.58 & 270 & 106 & 198 & 10 & 192 & 2 & 231 & 5 & 192 & 4 \\
\hline 0TQ15-53 & 0.0509 & 0.0031 & 0.0986 & 0.0059 & 0.0141 & 0.0002 & .0058 & 0.0002 & 2.05 & 234 & 112 & 96 & 5 & 90 & 1 & 117 & 3 & 90 & 2 \\
\hline $5-54$ & 0.0501 & 0.0034 & 0.0511 & 0.0034 & 0.0074 & 0. & 26 & 0.0000 & 0.71 & 201 & 131 & 51 & 3 & 47.5 & 0.6 & 53.1 & 0.8 & 47.5 & 1.2 \\
\hline 10TQ15-56 & 0.0484 & 0.0019 & 0.0898 & 0.0034 & 0.0135 & 0.0001 & 0.0047 & 0.0001 & 0.98 & 120 & 72 & 87 & 3 & 86.2 & 0.8 & 95 & 1 & 86.2 & 1.6 \\
\hline 10TQ15-57 & 0.0496 & 0.0132 & 0.0944 & 0.0246 & 0.0138 & 0.0007 & 0.0046 & 0.0006 & 1.82 & 178 & 389 & 92 & 23 & 88 & 4 & 92 & 12 & 88 & 8 \\
\hline $15-58$ & 0.0585 & 0.0007 & 0.5149 & 0.0047 & 0.0638 & & & 0.0001 & 2.97 & 550 & 10 & 422 & 3 & 399 & 2 & 368 & 3 & 399 & 4 \\
\hline 10TQ15-59 & 0.0834 & 0.0021 & 2.4028 & 0.0566 & 0.2090 & 0.0021 & 0.0700 & 0.0012 & 1.52 & 1279 & 30 & 1243 & 17 & 1223 & 11 & 1367 & 22 & 1279 & 60 \\
\hline 10TQ15-60 & 0.0986 & 0.0018 & 3.7722 & 0.0615 & 0.2775 & 0.0024 & 0.0865 & 0.0013 & 2.09 & 1598 & 18 & 1587 & 13 & 1579 & 12 & 1676 & 24 & 1598 & 36 \\
\hline OTQ15-61 & 0.0484 & 0.0039 & 0.0954 & 0.0074 & 0.0143 & 0.0002 & 0.0047 & 0.0002 & 1.72 & 120 & 144 & 93 & 7 & 91 & 1 & 94 & 3 & 91 & 2 \\
\hline 10TQ15-62 & 0.0495 & 0.0054 & 0.1130 & 0.0122 & 0.0166 & 0.0003 & 0.0071 & 0.0004 & 3.16 & 171 & 211 & 109 & 11 & 106 & 2 & 144 & 8 & 106 & 4 \\
\hline 10TQ15-63 & 0.0514 & 0.0095 & 0.1110 & 0.0201 & 0.0157 & 0.0006 & 0.0062 & 0.0004 & 1.08 & 258 & 310 & 107 & 18 & 100 & 4 & 125 & 8 & 100 & 8 \\
\hline 10TQ15-64 & 0.0481 & 0.0103 & 0.0864 & 0.0184 & 0.0130 & 0.0004 & 0.0053 & 0.0004 & 1.55 & 103 & 333 & 84 & 17 & 84 & 3 & 106 & 8 & 84 & 6 \\
\hline 10TQ15-65 & 0.1256 & 0.0017 & 6.4227 & 0.0711 & 0.3709 & 0.0028 & 0.1175 & 0.0011 & 1.26 & 2038 & 10 & 2035 & 10 & 2034 & 13 & 2245 & 20 & 2034 & 26 \\
\hline 10TQ15-66 & 0.0461 & 0.0399 & 0.0185 & 0.0159 & 0.0029 & 0.0003 & 0.0055 & 0.0032 & 2.73 & & 1131 & 19 & 16 & 19 & 2 & 111 & 64 & 19 & 4 \\
\hline 10TQ15-67 & 0.0495 & 0.0043 & 0.1396 & 0.0120 & 0.0205 & 0.0003 & 0.0074 & 0.0002 & 1.13 & 172 & 165 & 133 & 11 & 130 & 2 & 149 & 4 & 130 & 4 \\
\hline 10TQ15-68 & 0.0489 & 0.0080 & 0.1011 & 0.0163 & 0.0150 & 0.0004 & 0.0061 & 0.0004 & 1.87 & 141 & 277 & 98 & 15 & 96 & 3 & 124 & 8 & 96 & 6 \\
\hline 10TQ15-69 & 0.0518 & 0.0051 & 0.1037 & 0.0100 & 0.0145 & 0.0003 & 0.0054 & 0.0002 & 1.47 & 276 & 179 & 100 & 9 & 93 & 2 & 109 & 4 & 93 & 4 \\
\hline 10TQ15-70 & 0.0509 & 0.0110 & 0.1038 & 0.0220 & 0.0148 & 0.0006 & 0.0074 & 0.0005 & 1.33 & 236 & 342 & 100 & 20 & 95 & 4 & 149 & 11 & 95 & 8 \\
\hline
\end{tabular}

2010TQ07 (Dazuqu, Qiabulin section, $\mathrm{n}=107, \mathrm{~N}^{\circ}{ }^{\circ} 19.902^{\prime}$, E88 ${ }^{\circ} 30.784^{\prime}$, $4153 \mathrm{~m}$ )

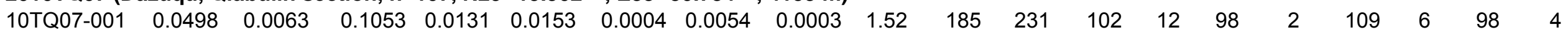


10TQ07-002 $0.0521 \quad 0.0020$ 10TQ07-003 $0.0505 \quad 0.0125$ $\begin{array}{lll}\text { 10TQ07-004 } & 0.0507 & 0.0045\end{array}$ $\begin{array}{lll}10 T Q 07-005 & 0.0781 & 0.0012\end{array}$ 10TQ07-006 $0.0519 \quad 0.0043$ $\begin{array}{lll}10 T Q 07-007 & 0.0503 & 0.0022\end{array}$ 10TQ07-008 $0.0512 \quad 0.0032$ $\begin{array}{lll}10 T Q 07-009 & 0.0492 & 0.0162\end{array}$ $\begin{array}{lll}10 T Q 07-010 & 0.0523 & 0.0032\end{array}$ $\begin{array}{lll}10 T Q 07-011 & 0.0523 & 0.0027\end{array}$ $\begin{array}{lll}10 T Q 07-012 & 0.0518 & 0.0121\end{array}$ 10TQ07-013 $0.0526 \quad 0.0030$ $\begin{array}{lll}10 T Q 07-014 & 0.0494 & 0.0026\end{array}$ $\begin{array}{lll}10 T Q 07-015 & 0.0775 & 0.0014\end{array}$ $\begin{array}{lll}\text { 10TQ07-016 } & 0.0530 & 0.0014\end{array}$ $\begin{array}{lll}10 T Q 07-017 & 0.0498 & 0.0034\end{array}$ $\begin{array}{lll}10 T Q 07-018 & 0.0462 & 0.0022\end{array}$ $\begin{array}{lll}\text { 10TQ07-019 } & 0.1889 & 0.0023\end{array}$ $\begin{array}{lll}10 T Q 07-020 & 0.0561 & 0.0009\end{array}$ $\begin{array}{lll}10 T Q 07-021 & 0.0500 & 0.0144\end{array}$ $\begin{array}{lll}\text { 10TQ07-022 } & 0.0527 & 0.0060\end{array}$ $\begin{array}{lll}\text { 10TQ07-023 } & 0.0496 & 0.0019\end{array}$ $\begin{array}{lll}\text { 10TQ07-024 } & 0.0478 & 0.0020\end{array}$ $\begin{array}{lll}\text { 10TQ07-025 } & 0.0496 & 0.0037\end{array}$ $\begin{array}{lll}\text { 10TQ07-026 } & 0.0506 & 0.0068\end{array}$ $\begin{array}{lll}10 T Q 07-027 & 0.0510 & 0.0036\end{array}$ 10TQ07-028 $0.0507 \quad 0.0029$ $\begin{array}{lll}10 T Q 07-029 & 0.0511 & 0.0031\end{array}$ 10TQ07-030 $0.0516 \quad 0.0056$ $\begin{array}{lll}10 T Q 07-031 & 0.0508 & 0.0082\end{array}$ $\begin{array}{lll}\text { 10TQ07-032 } & 0.0519 & 0.0075\end{array}$ $\begin{array}{lll}10 T Q 07-033 & 0.0491 & 0.0081\end{array}$ 10TQ07-034 $0.0499 \quad 0.0029$ 10TQ07-035 $0.0560 \quad 0.0019$ $\begin{array}{lllllll}0.2186 & 0.0078 & 0.0304 & 0.0003 & 0.0106 & 0.0002 & 1.49\end{array}$ $\begin{array}{lllllll}0.0555 & 0.0136 & 0.0080 & 0.0003 & 0.0029 & 0.0002 & 1.08\end{array}$ $\begin{array}{llllllll}0.0990 & 0.0087 & 0.0142 & 0.0002 & 0.0048 & 0.0002 & 1.21\end{array}$ $\begin{array}{llllllll}2.0784 & 0.0278 & 0.1930 & 0.0014 & 0.0649 & 0.0008 & 2.89\end{array}$ $\begin{array}{llllllll}0.1087 & 0.0089 & 0.0152 & 0.0003 & 0.0054 & 0.0002 & 1.49\end{array}$ $\begin{array}{lllllll}0.1032 & 0.0044 & 0.0149 & 0.0002 & 0.0049 & 0.0001 & 1.79\end{array}$ $\begin{array}{lllllll}0.0908 & 0.0056 & 0.0129 & 0.0002 & 0.0045 & 0.0001 & 1.56\end{array}$ $\begin{array}{lllllll}0.1010 & 0.0332 & 0.0149 & 0.0005 & 0.0066 & 0.0006 & 1.57\end{array}$ $\begin{array}{lllllll}0.1020 & 0.0060 & 0.0142 & 0.0002 & 0.0051 & 0.0001 & 1.03\end{array}$ $\begin{array}{lllllll}0.1018 & 0.0052 & 0.0141 & 0.0002 & 0.0047 & 0.0001 & 1.55\end{array}$ $\begin{array}{llllllll}0.0947 & 0.0219 & 0.0133 & 0.0005 & 0.0048 & 0.0005 & 1.79\end{array}$ $\begin{array}{lllllll}0.2114 & 0.0118 & 0.0291 & 0.0004 & 0.0097 & 0.0003 & 1.61\end{array}$ $\begin{array}{llllllll}0.0386 & 0.0020 & 0.0057 & 0.0001 & 0.0019 & 0.0000 & 1.23\end{array}$ $\begin{array}{lllllll}1.6378 & 0.0266 & 0.1532 & 0.0011 & 0.0460 & 0.0003 & 3.18\end{array}$ $\begin{array}{llllllll}0.1034 & 0.0026 & 0.0141 & 0.0001 & 0.0048 & 0.0001 & 1.10\end{array}$ $\begin{array}{lllllll}0.0943 & 0.0063 & 0.0138 & 0.0002 & 0.0044 & 0.0001 & 1.13\end{array}$ $\begin{array}{lllllll}0.0898 & 0.0042 & 0.0141 & 0.0002 & 0.0045 & 0.0001 & 1.50\end{array}$ $\begin{array}{lllllll}13.5391 & 0.1233 & 0.5200 & 0.0038 & 0.1445 & 0.0012 & 1.18\end{array}$ $\begin{array}{lllllll}0.5111 & 0.0072 & 0.0660 & 0.0005 & 0.0214 & 0.0002 & 1.00\end{array}$ $\begin{array}{llllllll}0.0957 & 0.0271 & 0.0139 & 0.0008 & 0.0073 & 0.0007 & 1.53\end{array}$ $\begin{array}{lllllll}0.2184 & 0.0245 & 0.0301 & 0.0005 & 0.0105 & 0.0004 & 1.78\end{array}$ $\begin{array}{lllllll}0.0985 & 0.0037 & 0.0144 & 0.0001 & 0.0048 & 0.0001 & 1.30\end{array}$ $\begin{array}{llllllll}0.0525 & 0.0022 & 0.0080 & 0.0001 & 0.0026 & 0.0000 & 0.69\end{array}$ $\begin{array}{llllllll}0.0571 & 0.0042 & 0.0084 & 0.0001 & 0.0027 & 0.0001 & 0.99\end{array}$ $\begin{array}{lllllll}0.1066 & 0.0141 & 0.0153 & 0.0004 & 0.0052 & 0.0003 & 1.12\end{array}$ $\begin{array}{llllllll}0.0981 & 0.0068 & 0.0139 & 0.0002 & 0.0047 & 0.0001 & 1.04\end{array}$ $\begin{array}{lllllll}0.1040 & 0.0057 & 0.0149 & 0.0002 & 0.0049 & 0.0001 & 0.96\end{array}$ $\begin{array}{llllllll}0.0552 & 0.0033 & 0.0078 & 0.0001 & 0.0025 & 0.0001 & 0.73\end{array}$ $\begin{array}{lllllll}0.1129 & 0.0119 & 0.0159 & 0.0004 & 0.0053 & 0.0002 & 0.68\end{array}$ $\begin{array}{lllllll}0.1036 & 0.0164 & 0.0148 & 0.0005 & 0.0053 & 0.0003 & 1.24\end{array}$ $\begin{array}{lllllll}0.1264 & 0.0181 & 0.0177 & 0.0004 & 0.0065 & 0.0003 & 1.34\end{array}$ $\begin{array}{llllllll}0.0526 & 0.0085 & 0.0078 & 0.0003 & 0.0028 & 0.0002 & 1.02\end{array}$ $\begin{array}{llllllll}0.0463 & 0.0026 & 0.0067 & 0.0001 & 0.0021 & 0.0000 & 0.58\end{array}$ $\begin{array}{llllllll}0.4526 & 0.0150 & 0.0587 & 0.0006 & 0.0196 & 0.0004 & 2.36\end{array}$

$\begin{array}{cccccccccc}290 & 64 & 201 & 7 & 193 & 2 & 213 & 4 & 193 & 4 \\ 219 & 395 & 55 & 13 & 51 & 2 & 58 & 4 & 51 & 4 \\ 228 & 170 & 96 & 8 & 91 & 1 & 98 & 3 & 91 & 2 \\ 1150 & 15 & 1142 & 9 & 1138 & 8 & 1272 & 15 & 1150 & 30 \\ 281 & 154 & 105 & 8 & 97 & 2 & 110 & 4 & 97 & 4 \\ 208 & 80 & 100 & 4 & 95 & 1 & 100 & 2 & 95 & 2 \\ 250 & 117 & 88 & 5 & 82 & 1 & 90 & 3 & 82 & 2 \\ 156 & 502 & 98 & 31 & 95 & 3 & 132 & 12 & 95 & 6 \\ 299 & 109 & 99 & 6 & 91 & 1 & 102 & 2 & 91 & 2 \\ 296 & 94 & 98 & 5 & 90 & 1 & 95 & 2 & 90 & 2 \\ 277 & 380 & 92 & 20 & 85 & 3 & 96 & 9 & 85 & 6 \\ 312 & 103 & 195 & 10 & 185 & 2 & 194 & 5 & 185 & 4 \\ 165 & 97 & 38 & 2 & 36.4 & 0.4 & 39.2 & 0.8 & 36.4 & 0.8 \\ 1134 & 36 & 985 & 10 & 919 & 6 & 909 & 6 & 919 & 12 \\ 330 & 42 & 100 & 2 & 90.5 & 0.7 & 97 & 1 & 90.5 & 1.4 \\ 183 & 125 & 91 & 6 & 88 & 1 & 88 & 2 & 88 & 2 \\ 5 & 104 & 87 & 4 & 90.4 & 1 & 91 & 2 & 90.4 & 2 \\ 2733 & 7 & 2718 & 9 & 2699 & 16 & 2728 & 21 & 2733 & 14 \\ 458 & 19 & 419 & 5 & 412 & 3 & 427 & 3 & 412 & 6 \\ 195 & 420 & 93 & 25 & 89 & 5 & 147 & 15 & 89 & 10 \\ 316 & 226 & 201 & 20 & 191 & 3 & 212 & 7 & 191 & 6 \\ 178 & 70 & 95 & 3 & 92.1 & 0.9 & 96 & 2 & 92.1 & 1.8 \\ 88 & 73 & 52 & 2 & 51.2 & 0.5 & 52.7 & 0.6 & 51.2 & 1 \\ 178 & 145 & 56 & 4 & 53.6 & 0.6 & 55 & 1 & 53.6 & 1.2 \\ 220 & 245 & 103 & 13 & 98 & 3 & 104 & 5 & 98 & 6 \\ 242 & 133 & 95 & 6 & 89 & 1 & 94 & 2 & 89 & 2 \\ 227 & 104 & 100 & 5 & 95 & 1 & 99 & 2 & 95 & 2 \\ 244 & 111 & 55 & 3 & 50.3 & 0.7 & 51 & 1 & 50.3 & 1.4 \\ 269 & 197 & 109 & 11 & 101 & 2 & 107 & 3 & 101 & 4 \\ 229 & 279 & 100 & 15 & 95 & 3 & 106 & 6 & 95 & 6 \\ 282 & 278 & 121 & 16 & 113 & 3 & 132 & 6 & 113 & 6 \\ 151 & 271 & 52 & 8 & 50 & 2 & 56 & 3 & 50 & 4 \\ 188 & 109 & 46 & 3 & 43.2 & 0.5 & 43.2 & 0.6 & 43.2 & 1 \\ 450 & 56 & 379 & 10 & 368 & 3 & 393 & 8 & 368 & 6\end{array}$


$\begin{array}{lll}\text { 10TQ07-036 } & 0.0476 & 0.0021\end{array}$ $\begin{array}{lll}10 T Q 07-037 & 0.0461 & 0.0025\end{array}$ $\begin{array}{lll}\text { 10TQ07-038 } & 0.0500 & 0.0112\end{array}$ 10TQ07-039 $0.0483 \quad 0.0045$

$\begin{array}{lll}10 T Q 07-040 & 0.0501 & 0.0032\end{array}$ $\begin{array}{lll}10 T Q 07-041 & 0.0524 & 0.0016\end{array}$

$\begin{array}{lll}\text { 10TQ07-042 } & 0.0481 & 0.0045\end{array}$ $\begin{array}{lll}10 T Q 07-043 & 0.0512 & 0.0026\end{array}$

$\begin{array}{lll}10 T Q 07-044 & 0.0513 & 0.0031\end{array}$ $\begin{array}{lll}10 T Q 07-045 & 0.0508 & 0.0034\end{array}$ $\begin{array}{lll}10 T Q 07-046 & 0.0504 & 0.0045\end{array}$ 10TQ07-047 $0.0481 \quad 0.0034$ 10TQ07-048 $0.0504 \quad 0.0026$ $\begin{array}{lll}\text { 10TQ07-049 } & 0.1071 & 0.0013\end{array}$ $\begin{array}{lll}10 T Q 07-050 & 0.0487 & 0.0065\end{array}$ $\begin{array}{lll}\text { 10TQ07-051 } & 0.0543 & 0.0008\end{array}$ 10TQ07-052 $0.0496 \quad 0.0048$ $\begin{array}{lll}\text { 10TQ07-053 } & 0.0505 & 0.0034\end{array}$ $\begin{array}{lll}10 T Q 07-054 & 0.0506 & 0.0078\end{array}$ $\begin{array}{lll}\text { 10TQ07-055 } & 0.0494 & 0.0059\end{array}$ $\begin{array}{lll}10 T Q 07-056 & 0.0502 & 0.0083\end{array}$ $\begin{array}{lll}10 T Q 07-057 & 0.0518 & 0.0054\end{array}$ $\begin{array}{lll}10 T Q 07-058 & 0.0553 & 0.0017\end{array}$ $\begin{array}{lll}\text { 10TQ07-059 } & 0.0525 & 0.0041\end{array}$ $\begin{array}{lll}10 T Q 07-060 & 0.0509 & 0.0075\end{array}$ $\begin{array}{lll}\text { 10TQ07-061 } & 0.0695 & 0.0010\end{array}$ $\begin{array}{lll}\text { 10TQ07-062 } & 0.0493 & 0.0019\end{array}$ $\begin{array}{lll}\text { 10TQ07-063 } & 0.0487 & 0.0045\end{array}$ $\begin{array}{lll}10 T Q 07-064 & 0.0513 & 0.0031\end{array}$ $\begin{array}{lll}10 T Q 07-065 & 0.0502 & 0.0014\end{array}$ $\begin{array}{lll}\text { 10TQ07-066 } & 0.0524 & 0.0050\end{array}$ $\begin{array}{lll}10 T Q 07-067 & 0.0494 & 0.0016\end{array}$ $\begin{array}{lll}10 T Q 07-068 & 0.1092 & 0.0013\end{array}$ $\begin{array}{lll}\text { 10TQ07-069 } & 0.0526 & 0.0024\end{array}$ $\begin{array}{lllllll}0.0543 & 0.0023 & 0.0083 & 0.0001 & 0.0028 & 0.0001 & 1.97\end{array}$ $\begin{array}{lllllll}0.0919 & 0.0047 & 0.0145 & 0.0002 & 0.0053 & 0.0004 & 0.92\end{array}$ $\begin{array}{lllllll}0.0911 & 0.0202 & 0.0132 & 0.0004 & 0.0042 & 0.0003 & 1.11\end{array}$ $\begin{array}{lllllll}0.0954 & 0.0087 & 0.0143 & 0.0002 & 0.0045 & 0.0002 & 1.32\end{array}$ $\begin{array}{lllllll}0.0498 & 0.0031 & 0.0072 & 0.0001 & 0.0025 & 0.0001 & 1.03\end{array}$ $\begin{array}{lllllll}0.1089 & 0.0032 & 0.0151 & 0.0001 & 0.0050 & 0.0001 & 0.67\end{array}$ $\begin{array}{lllllll}0.0879 & 0.0081 & 0.0133 & 0.0002 & 0.0043 & 0.0002 & 1.29\end{array}$ $\begin{array}{lllllll}0.1011 & 0.0049 & 0.0143 & 0.0002 & 0.0056 & 0.0002 & 3.80\end{array}$ $\begin{array}{lllllll}0.0574 & 0.0034 & 0.0081 & 0.0001 & 0.0026 & 0.0001 & 1.02\end{array}$ $\begin{array}{lllllll}0.1950 & 0.0130 & 0.0278 & 0.0004 & 0.0091 & 0.0003 & 1.57\end{array}$ $\begin{array}{llllllll}0.0946 & 0.0083 & 0.0136 & 0.0002 & 0.0045 & 0.0001 & 1.17\end{array}$ $\begin{array}{llllllll}0.0508 & 0.0035 & 0.0077 & 0.0001 & 0.0026 & 0.0001 & 1.09\end{array}$ $\begin{array}{lllllll}0.1005 & 0.0050 & 0.0145 & 0.0002 & 0.0050 & 0.0001 & 1.73\end{array}$ $\begin{array}{llllllll}4.6910 & 0.0441 & 0.3178 & 0.0022 & 0.0940 & 0.0008 & 1.77\end{array}$ $\begin{array}{lllllll}0.0826 & 0.0109 & 0.0123 & 0.0003 & 0.0045 & 0.0002 & 1.58\end{array}$ $\begin{array}{lllllll}0.4009 & 0.0049 & 0.0536 & 0.0004 & 0.0131 & 0.0001 & 0.79\end{array}$ $\begin{array}{lllllll}0.0978 & 0.0093 & 0.0143 & 0.0002 & 0.0052 & 0.0001 & 1.37\end{array}$ $\begin{array}{lllllll}0.0990 & 0.0065 & 0.0142 & 0.0002 & 0.0050 & 0.0001 & 1.27\end{array}$ $\begin{array}{lllllll}0.0563 & 0.0086 & 0.0081 & 0.0002 & 0.0033 & 0.0002 & 1.78\end{array}$ $\begin{array}{lllllll}0.0537 & 0.0063 & 0.0079 & 0.0001 & 0.0026 & 0.0001 & 1.09\end{array}$ $\begin{array}{lllllll}0.0962 & 0.0157 & 0.0139 & 0.0004 & 0.0049 & 0.0003 & 1.23\end{array}$ $\begin{array}{lllllll}0.1120 & 0.0117 & 0.0157 & 0.0002 & 0.0052 & 0.0001 & 1.09\end{array}$ $\begin{array}{lllllll}0.4424 & 0.0129 & 0.0580 & 0.0005 & 0.0181 & 0.0001 & 1.78\end{array}$ $\begin{array}{llllllll}0.1077 & 0.0083 & 0.0149 & 0.0002 & 0.0049 & 0.0002 & 1.80\end{array}$ $\begin{array}{lllllll}0.0560 & 0.0082 & 0.0080 & 0.0002 & 0.0031 & 0.0002 & 1.42\end{array}$ $\begin{array}{llllllll}1.2344 & 0.0150 & 0.1289 & 0.0009 & 0.0398 & 0.0006 & 5.66\end{array}$ $\begin{array}{lllllll}0.0882 & 0.0033 & 0.0130 & 0.0001 & 0.0045 & 0.0001 & 1.30\end{array}$ $\begin{array}{lllllll}0.0893 & 0.0081 & 0.0133 & 0.0002 & 0.0044 & 0.0001 & 1.15\end{array}$ $\begin{array}{lllllll}0.0530 & 0.0032 & 0.0075 & 0.0001 & 0.0023 & 0.0000 & 0.58\end{array}$ $\begin{array}{lllllll}0.0975 & 0.0026 & 0.0141 & 0.0001 & 0.0048 & 0.0001 & 1.19\end{array}$ $\begin{array}{lllllll}0.1086 & 0.0101 & 0.0150 & 0.0003 & 0.0061 & 0.0003 & 1.84\end{array}$ $\begin{array}{llllllll}0.0962 & 0.0030 & 0.0141 & 0.0001 & 0.0044 & 0.0001 & 0.66\end{array}$ $\begin{array}{llllllll}4.6255 & 0.0378 & 0.3074 & 0.0020 & 0.0893 & 0.0008 & 4.61\end{array}$ $\begin{array}{lllllll}0.1307 & 0.0058 & 0.0180 & 0.0002 & 0.0057 & 0.0001 & 1.25\end{array}$

$\begin{array}{cccccccccc}78 & 77 & 54 & 2 & 53.2 & 0.5 & 57 & 1 & 53.2 & 1 \\ & 116 & 89 & 4 & 93 & 2 & 107 & 8 & 93 & 4 \\ 193 & 360 & 89 & 19 & 85 & 3 & 85 & 6 & 85 & 6 \\ 113 & 171 & 93 & 8 & 92 & 2 & 90 & 3 & 92 & 4 \\ 201 & 118 & 49 & 3 & 46.2 & 0.6 & 50 & 1 & 46.2 & 1.2 \\ 302 & 51 & 105 & 3 & 96.5 & 0.8 & 100 & 1 & 96.5 & 1.6 \\ 105 & 175 & 86 & 8 & 85 & 1 & 87 & 3 & 85 & 2 \\ 249 & 90 & 98 & 5 & 92 & 1 & 112 & 4 & 92 & 2 \\ 255 & 115 & 57 & 3 & 52.1 & 0.6 & 52 & 1 & 52.1 & 1.2 \\ 232 & 127 & 181 & 11 & 177 & 2 & 183 & 5 & 177 & 4 \\ 212 & 167 & 92 & 8 & 87 & 1 & 90 & 3 & 87 & 2 \\ 106 & 128 & 50 & 3 & 49.1 & 0.6 & 53 & 1 & 49.1 & 1.2 \\ 211 & 94 & 97 & 5 & 93 & 1 & 101 & 3 & 93 & 2 \\ 1750 & 8 & 1766 & 8 & 1779 & 11 & 1816 & 14 & 1750 & 16 \\ 133 & 244 & 81 & 10 & 79 & 2 & 90 & 4 & 79 & 4 \\ 381 & 16 & 342 & 4 & 337 & 2 & 263 & 2 & 337 & 4 \\ 178 & 188 & 95 & 9 & 92 & 1 & 105 & 3 & 92 & 2 \\ 217 & 122 & 96 & 6 & 91 & 1 & 100 & 3 & 91 & 2 \\ 222 & 284 & 56 & 8 & 52 & 1 & 66 & 4 & 52 & 2 \\ 166 & 231 & 53 & 6 & 50.7 & 0.8 & 53 & 2 & 50.7 & 1.6 \\ 203 & 286 & 93 & 15 & 89 & 3 & 98 & 6 & 89 & 6 \\ 275 & 210 & 108 & 11 & 100 & 1 & 105 & 3 & 100 & 2 \\ 424 & 69 & 372 & 9 & 364 & 3 & 363 & 2 & 364 & 6 \\ 305 & 149 & 104 & 8 & 95 & 1 & 99 & 4 & 95 & 2 \\ 235 & 270 & 55 & 8 & 51 & 1 & 63 & 3 & 51 & 2 \\ 913 & 14 & 816 & 7 & 782 & 5 & 788 & 11 & 782 & 10 \\ 162 & 68 & 86 & 3 & 83.1 & 0.8 & 90 & 1 & 83.1 & 1.6 \\ 134 & 172 & 87 & 8 & 85 & 1 & 89 & 3 & 85 & 2 \\ 252 & 112 & 52 & 3 & 48.2 & 0.6 & 46.8 & 0.8 & 48.2 & 1.2 \\ 205 & 47 & 94 & 2 & 90.2 & 0.8 & 96 & 1 & 90.2 & 1.6 \\ 302 & 176 & 105 & 9 & 96 & 2 & 122 & 5 & 96 & 4 \\ 167 & 56 & 93 & 3 & 90.5 & 0.8 & 90 & 1 & 90.5 & 1.6 \\ 1786 & 7 & 1754 & 7 & 1728 & 10 & 1729 & 16 & 1786 & 14 \\ 312 & 81 & 125 & 5 & 115 & 1 & 114 & 2 & 115 & 2\end{array}$




\begin{tabular}{|c|c|c|c|c|c|c|c|c|c|c|c|c|c|c|c|c|c|c|c|}
\hline 10TQ07-070 & 0.0462 & 0.0035 & 0.0903 & 0.0066 & 0.0142 & 0.0002 & 0.0052 & 0.0002 & 2.74 & 8 & 133 & 88 & 6 & 91 & 1 & 105 & 4 & 91 & 2 \\
\hline 10TQ07-071 & 0.0517 & 0.0020 & 0.1043 & 0.0038 & 0.0146 & 0.0001 & 0.0046 & 0.0001 & 0.67 & 272 & 65 & 101 & 3 & 93.7 & 0.9 & 93 & 1 & 93.7 & 1.8 \\
\hline OTQ07-072 & 0.0518 & 0.0020 & 0.0691 & 0.0026 & 0.0097 & 0.0001 & 0.0033 & 0.0001 & 1.39 & 278 & 69 & 68 & 0 & 62 & 0.6 & 67 & 1 & 62 & 1.2 \\
\hline OTQ07-073 & 0.0487 & 0.0061 & 0.0509 & 0.0063 & 0.0076 & 0.0002 & 0.0023 & 0.0001 & 1.69 & 135 & 230 & 50 & 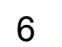 & 49 & 1 & 47 & 3 & 49 & 2 \\
\hline OTQ07-074 & 0.0504 & 0.0035 & 0.0997 & 0.0068 & 0.0144 & 0.0002 & 0.0045 & 0.0001 & 0.99 & 212 & 129 & 96 & 6 & 92 & 1 & 91 & 2 & 92 & \\
\hline OTQ07-075 & 0.0505 & 0.0037 & 0.0550 & 0.0039 & 0.0079 & 0.0001 & 0.0026 & 0.0001 & 1.33 & 216 & 135 & 54 & 4 & 50.7 & 0.8 & 53 & 2 & 50.7 & 6 \\
\hline DTQ07-076 & 0.0482 & 0.0040 & 0561 & 0.0045 & 0.0084 & 0.0001 & 0.0028 & 0.0001 & 1.16 & 111 & 151 & 55 & 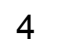 & 4.2 & 0.8 & 56 & 2 & 4.2 & 6 \\
\hline DTQ07-077 & 0.0499 & 0013 & 0974 & 0.0023 & 0.0142 & 0.0001 & 0.0051 & 0.0001 & 6.72 & 189 & 40 & 94 & 2 & 90.7 & 0.7 & 103 & 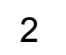 & 90.7 & \\
\hline DTQ07-078 & 0.0478 & 0104 & 36 & 0.0115 & 0.0081 & 0.0002 & 0.0025 & 0001 & .61 & 89 & 337 & 53 & 11 & 52 & 4 & 50 & & 52 & \\
\hline OTQ07-079 & 0.0490 & 0062 & 0.0532 & 0.0066 & 0.0079 & .0002 & 0.0027 & 0001 & 1.64 & 146 & 238 & 53 & & 51 & 1 & 54 & & 51 & \\
\hline OTQ07-080 & 0.0522 & 0.0045 & 0.2202 & 0.0188 & 0.0306 & 0.0005 & 0.0094 & 0.0004 & 1.31 & 293 & 162 & 202 & 16 & 194 & 3 & 189 & 7 & 194 & \\
\hline OTQ07-081 & 0.0461 & 0.0038 & 0.0480 & 0.0038 & 0.0076 & 0.0002 & 0.0035 & 0.0005 & 1.29 & & 179 & 48 & 4 & 48.5 & 1 & 71 & 11 & 48.5 & \\
\hline DTQ07-082 & 0.0499 & 0.0041 & 0.0954 & 0.0078 & 0.0139 & 0.0002 & 0.0045 & 0.0001 & 1.03 & 188 & 156 & 93 & 7 & 89 & 1 & 91 & 2 & 89 & \\
\hline 0TQ07-083 & 0.0698 & 0.0018 & 1.3884 & 0.0339 & 0.1442 & 0.0012 & 0.0438 & 0.0003 & 1.96 & 924 & 54 & 884 & 14 & 868 & 7 & 866 & 6 & 868 & 4 \\
\hline OTQ07-084 & 0.0504 & 0.0037 & 0.1029 & 0.0074 & 0.0148 & 0.0002 & 0.0048 & 0.0002 & 1.38 & 212 & 136 & 99 & 7 & 95 & 1 & 98 & 3 & 95 & \\
\hline 0TQ07-085 & 0.0517 & 0.0061 & 0.2247 & 0.0259 & 0.0315 & 0.0007 & 0.0118 & 0.0006 & 1.52 & 274 & 217 & 206 & 21 & 200 & 5 & 236 & 11 & 200 & 10 \\
\hline-086 & 0.0488 & 0.0045 & 0.1126 & 0.0103 & 0.0167 & 0.0003 & 0.0052 & 0.0002 & 1.15 & 138 & 172 & 108 & 0 & 107 & 2 & 105 & 3 & 107 & \\
\hline 7-087 & 0.0619 & 0.0014 & 0.9084 & 0.0184 & 0.1064 & 0.0009 & 0.0325 & 0.0003 & 1.06 & 672 & 29 & 656 & 10 & 652 & 5 & 646 & 7 & 652 & U \\
\hline 10TQ07-088 & 0.0762 & 0.0010 & 2.0190 & 0.0203 & 0.1921 & 0.0013 & 0.0558 & 0.0005 & 2.16 & 1101 & 10 & 1122 & 7 & 1133 & 7 & 1098 & 9 & 1101 & 20 \\
\hline 10TQ07-089 & 0.0497 & 0.0094 & 0.0971 & 0.0182 & 0.0142 & 0.0004 & 0.0042 & 0.0003 & 1.53 & 180 & 313 & 94 & 17 & 91 & 3 & 85 & 7 & 91 & 6 \\
\hline 10TQ07-090 & 0.0824 & 0.0014 & 2.3154 & 0.0358 & 0.2040 & 0.0016 & 0.0612 & 0.0005 & 0.83 & 1254 & 18 & 1217 & 11 & 1197 & 9 & 1200 & 10 & 1254 & 36 \\
\hline 7-091 & 0.0550 & 0.0011 & 0.4526 & 0.0079 & 0.0597 & 0.0004 & 0.0182 & 0.0003 & 4.26 & 412 & 26 & 379 & 6 & 374 & 3 & 364 & 6 & 374 & 6 \\
\hline 10TQ07-092 & 0.0931 & 0.0013 & 3.4436 & 0.0384 & 0.2684 & 0.0019 & 0.0735 & 0.0007 & 1.52 & 1489 & 11 & 1514 & 9 & 1533 & 10 & 1434 & 12 & 1489 & 22 \\
\hline 10TQ & 0.0509 & 0.0022 & 0.0963 & 0.0040 & 0.0137 & 0.0001 & 0.0045 & 0.0001 & 1.50 & 234 & 77 & 93 & 4 & 88 & 0.9 & 92 & 2 & 88 & .8 \\
\hline 10TC & 0.0505 & 0.0022 & 0.0 & 0.0040 & 0.0 & 0.0 & 0.0 & 01 & 0.98 & 216 & 78 & 92 & 4 & 86.9 & 0.9 & 86 & 1 & 6.9 & 8 \\
\hline $10 \mathrm{TC}$ & 0.0 & 0 & & 0.0 & 0.0 & 06 & 1 & 04 & 4.83 & 559 & 20 & 561 & 6 & 562 & 4 & 560 & 8 & 2 & 8 \\
\hline-096 & 0.0497 & .0103 & .0566 & 0.0116 & 0.0083 & 0.0003 & 0.0029 & 0.0002 & 0.86 & 181 & 334 & 56 & 11 & 53 & 2 & 58 & 3 & 53 & \\
\hline 0TQ07-097 & 0.0461 & 0.0027 & 0.0484 & 0.0027 & 0.0076 & 0.0002 & 0.0030 & 0.0003 & 1.03 & & 130 & 48 & 3 & 48.9 & 1 & 61 & 6 & 48.9 & 2 \\
\hline 0TQ07-098 & 0.0498 & 0.0038 & .0556 & 0.0042 & 0.0081 & 0.0001 & 0.0026 & 0.0001 & 1.00 & 187 & 139 & 55 & 4 & 52 & 0.8 & 52 & 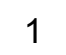 & 52 & 6 \\
\hline 0TQ07-099 & 0.0494 & 0.0154 & 0.0550 & 0.0171 & 0.0081 & 0.0003 & 0.0031 & 0.0002 & 0.85 & 168 & 481 & 54 & 16 & 52 & 2 & 62 & 4 & 52 & \\
\hline OTQ07-100 & 0.0510 & .0035 & 0.2247 & 0.0149 & 0.0320 & 0.0005 & 0.0110 & 0.0004 & 2.33 & 239 & 123 & 206 & 12 & 203 & 3 & 220 & 9 & 203 & 6 \\
\hline 10TQ07-101 & 0.0704 & 0.0015 & 1.4613 & 0.0273 & 0.1507 & 0.0012 & 0.0449 & 0.0006 & 1.57 & 939 & 25 & 915 & 11 & 905 & 7 & 887 & 11 & 905 & 14 \\
\hline OTQQ & 0.0527 & .0014 & 0.2271 & 0.0055 & 0.0312 & 0.0003 & 0.0097 & 0.0001 & 1.36 & 317 & 41 & 208 & 5 & 198 & 2 & 196 & 2 & 198 & \\
\hline OTQ07-103 & 0.0488 & 0.0037 & 0.0923 & 0.0068 & 0.0137 & 0.0002 & 0.0043 & 0.0001 & 1.16 & 136 & 139 & 90 & 6 & 88 & 1 & 86 & 2 & 88 & \\
\hline
\end{tabular}




\begin{tabular}{|c|c|c|c|c|c|c|c|c|c|c|c|c|c|c|c|c|c|c|c|}
\hline 10TQ07-104 & 0.0498 & 0.0097 & 0.1003 & 0.0193 & 0.0146 & 0.0004 & 0.0051 & 0.0004 & 1.64 & 184 & 317 & 97 & 18 & 94 & 3 & 104 & 8 & 94 & 6 \\
\hline 10TQ07-105 & 0.0514 & 0.0036 & 0.1008 & 0.0070 & 0.0142 & 0.0002 & 0.0044 & 0.0001 & 1.34 & 257 & 133 & 97 & 6 & 91 & 1 & 89 & 3 & 91 & 2 \\
\hline 10TQ07-106 & 0.0482 & 0.0009 & 0.0951 & 0.0015 & 0.0143 & 0.0001 & 0.0044 & 0.0001 & 2.36 & 109 & 25 & 92 & 1 & 91.7 & 0.6 & 89 & 1 & 91.7 & 1.2 \\
\hline 10TQ07-107 & 0.0461 & 0.0019 & 0.0544 & 0.0022 & 0.0086 & 0.0001 & 0.0027 & 0.0000 & 0.83 & 2 & 86 & 54 & 2 & 54.9 & 0.5 & 55.1 & 0.5 & 54.9 & 1 \\
\hline
\end{tabular}

2010TQ11 (Dazuqu, Qiabulin section, $\mathrm{n}=105, \mathrm{~N}^{2} 9^{\circ} 19.693^{\prime}$, E88 ${ }^{\circ} 31.533^{\prime}$, $3942 \mathrm{~m}$ ) $\begin{array}{lllllllllll}10 T Q 11-002 & 0.0507 & 0.0096 & 0.0998 & 0.0187 & 0.0143 & 0.0003 & 0.0049 & 0.0003 & 1.49\end{array}$

$\begin{array}{lllllllllll}10 T Q 11-003 & 0.0528 & 0.0107 & 0.1087 & 0.0216 & 0.0149 & 0.0006 & 0.0055 & 0.0004 & 1.29\end{array}$

$\begin{array}{llllllllll}10 T Q 11-004 & 0.0505 & 0.0058 & 0.0524 & 0.0060 & 0.0075 & 0.0001 & 0.0024 & 0.0001 & 1.16\end{array}$

$\begin{array}{llllllllll}10 T Q 11-005 & 0.0508 & 0.0020 & 0.1019 & 0.0038 & 0.0146 & 0.0001 & 0.0051 & 0.0001 & 1.28\end{array}$

$\begin{array}{llllllllll}\text { 10TQ11-006 } & 0.0788 & 0.0020 & 2.0320 & 0.0471 & 0.1872 & 0.0019 & 0.0637 & 0.0010 & 1.59\end{array}$

$\begin{array}{llllllllll}10 T Q 11-007 & 0.0501 & 0.0053 & 0.0514 & 0.0053 & 0.0074 & 0.0001 & 0.0024 & 0.0002 & 2.79\end{array}$

$\begin{array}{llllllllll}\text { 10TQ11-008 } & 0.0507 & 0.0057 & 0.0958 & 0.0106 & 0.0137 & 0.0003 & 0.0049 & 0.0002 & 1.50\end{array}$

$\begin{array}{llllllllll}\text { 10TQ11-009 } & 0.0499 & 0.0103 & 0.0925 & 0.0190 & 0.0134 & 0.0004 & 0.0057 & 0.0004 & 1.92\end{array}$

$\begin{array}{llllllllll}\text { 10TQ11-010 } & 0.0503 & 0.0036 & 0.0956 & 0.0068 & 0.0138 & 0.0002 & 0.0045 & 0.0001 & 0.69\end{array}$

$\begin{array}{llllllllll}10 T Q 11-011 & 0.0520 & 0.0051 & 0.1078 & 0.0105 & 0.0150 & 0.0002 & 0.0056 & 0.0002 & 1.04\end{array}$

$\begin{array}{lllllllllll}10 T Q 11-012 & 0.0505 & 0.0064 & 0.2001 & 0.0250 & 0.0288 & 0.0005 & 0.0095 & 0.0004 & 1.27\end{array}$

$\begin{array}{llllllllll}10 T Q 11-013 & 0.0615 & 0.0017 & 0.7966 & 0.0205 & 0.0941 & 0.0008 & 0.0300 & 0.0002 & 0.57\end{array}$

10TQ11-014 $0.0513 \quad 0.0095$

$\begin{array}{lll}10 T Q 11-015 & 0.0522 & 0.0063\end{array}$

$\begin{array}{lll}10 T Q 11-016 & 0.0486 & 0.0092\end{array}$

10TQ11-017 $0.0983 \quad 0.0011$

$\begin{array}{llllllll}0.1969 & 0.0362 & 0.0279 & 0.0006 & 0.0088 & 0.0006 & 1.60\end{array}$

$\begin{array}{lllllll}0.0990 & 0.0119 & 0.0138 & 0.0002 & 0.0053 & 0.0001 & 1.39\end{array}$

$\begin{array}{llllllll}0.0560 & 0.0105 & 0.0084 & 0.0002 & 0.0031 & 0.0001 & 0.80\end{array}$

$\begin{array}{llllllll}3.7150 & 0.0321 & 0.2741 & 0.0019 & 0.0811 & 0.0008 & 5.94\end{array}$

$\begin{array}{lll}10 T Q 11-018 & 0.0503 & 0.0048\end{array}$

10TQ11-019 $0.0467 \quad 0.0188$

$\begin{array}{lll}\text { 10TQ11-020 } & 0.0548 & 0.0020\end{array}$

$\begin{array}{lll}10 T Q 11-021 & 0.0498 & 0.0040\end{array}$

$\begin{array}{llllllll}0.1793 & 0.0170 & 0.0259 & 0.0003 & 0.0084 & 0.0002 & 1.04\end{array}$

$\begin{array}{lllllll}0.0544 & 0.0218 & 0.0084 & 0.0003 & 0.0027 & 0.0003 & 1.15\end{array}$

$\begin{array}{llllllll}0.2744 & 0.0098 & 0.0363 & 0.0004 & 0.0114 & 0.0002 & 1.81\end{array}$

$\begin{array}{lllllll}0.0538 & 0.0042 & 0.0078 & 0.0001 & 0.0026 & 0.0001 & 1.05\end{array}$

$\begin{array}{llllllll}0.4416 & 0.0094 & 0.0586 & 0.0004 & 0.0183 & 0.0001 & 3.07\end{array}$

10TQ11-022 $0.0547 \quad 0.0012$

$\begin{array}{lllllll}0.0981 & 0.0254 & 0.0141 & 0.0005 & 0.0062 & 0.0005 & 1.65\end{array}$

10TQ11-023 $0.0505 \quad 0.0132$

$\begin{array}{lllllll}1.6668 & 0.0270 & 0.1661 & 0.0013 & 0.0518 & 0.0005 & 1.38\end{array}$

$\begin{array}{lll}10 T Q 11-025 & 0.1086 & 0.0012\end{array}$

$\begin{array}{lllllll}4.9190 & 0.0395 & 0.3286 & 0.0022 & 0.1065 & 0.0007 & 2.57\end{array}$

$\begin{array}{lll}\text { 10TQ11-026 } & 0.0523 & 0.0048\end{array}$

$\begin{array}{lllllll}0.1166 & 0.0106 & 0.0162 & 0.0002 & 0.0051 & 0.0000 & 0.60\end{array}$

10TQ11-027 $0.0506 \quad 0.0105$

$\begin{array}{llllllll}0.0679 & 0.0139 & 0.0097 & 0.0003 & 0.0044 & 0.0003 & 1.42\end{array}$

$\begin{array}{cccccccccc}219 & 256 & 68 & 9 & 64 & 1 & 69 & 3 & 64 & 2 \\ 228 & 335 & 97 & 17 & 91 & 2 & 99 & 6 & 91 & 4 \\ 322 & 343 & 105 & 20 & 95 & 4 & 111 & 8 & 95 & 8 \\ 216 & 222 & 52 & 6 & 48.4 & 0.9 & 49 & 2 & 48.4 & 1.8 \\ 230 & 68 & 99 & 3 & 93.2 & 0.9 & 102 & 2 & 93.2 & 1.8 \\ 1167 & 30 & 1126 & 16 & 1106 & 10 & 1248 & 19 & 1167 & 60 \\ 199 & 202 & 51 & 5 & 47.8 & 0.8 & 49 & 3 & 47.8 & 1.6 \\ 229 & 216 & 93 & 10 & 88 & 2 & 99 & 4 & 88 & 4 \\ 192 & 339 & 90 & 18 & 86 & 2 & 114 & 8 & 86 & 4 \\ 209 & 139 & 93 & 6 & 88 & 1 & 90 & 2 & 88 & 2 \\ 286 & 190 & 104 & 10 & 96 & 2 & 112 & 3 & 96 & 4 \\ 216 & 250 & 185 & 21 & 183 & 3 & 190 & 7 & 183 & 6 \\ 655 & 41 & 595 & 12 & 580 & 4 & 598 & 5 & 580 & 8 \\ 252 & 354 & 183 & 31 & 177 & 4 & 176 & 11 & 177 & 8 \\ 295 & 249 & 96 & 11 & 88 & 1 & 107 & 3 & 88 & 2 \\ 127 & 308 & 55 & 10 & 54 & 1 & 63 & 3 & 54 & 2 \\ 1593 & 7 & 1575 & 7 & 1561 & 10 & 1576 & 15 & 1593 & 14 \\ 209 & 191 & 167 & 15 & 165 & 2 & 170 & 4 & 165 & 4 \\ 36 & 596 & 54 & 21 & 54 & 2 & 55 & 5 & 54 & 4 \\ 406 & 62 & 246 & 8 & 230 & 2 & 229 & 5 & 230 & 4 \\ 186 & 150 & 53 & 4 & 50.3 & 0.7 & 51 & 1 & 50.3 & 1.4 \\ 398 & 52 & 371 & 7 & 367 & 3 & 367 & 2 & 367 & 6 \\ 218 & 416 & 95 & 24 & 90 & 3 & 125 & 9 & 90 & 6 \\ 1008 & 20 & 996 & 10 & 991 & 7 & 1020 & 10 & 991 & 14 \\ 1776 & 7 & 1806 & 7 & 1832 & 11 & 2046 & 13 & 1776 & 14 \\ 297 & 210 & 112 & 10 & 103 & 1 & 102.4 & 0.8 & 103 & 2 \\ 224 & 335 & 67 & 13 & 62 & 2 & 89 & 6 & 62 & 4 \\ 227 & 187 & 116 & 11 & 110 & 2 & 123 & 5 & 110 & 4\end{array}$




\begin{tabular}{|c|c|c|c|c|c|c|c|c|c|c|c|c|c|c|c|c|c|c|c|}
\hline 10TQ11-029 & .0596 & 0.0014 & 0.7811 & 0.0175 & 0.0951 & 0.0008 & 0.0282 & 0.0002 & 0.40 & 589 & 34 & 586 & 10 & 585 & 5 & 563 & 4 & 585 & 10 \\
\hline 10TQ11-030 & 0.1093 & 0.0017 & 4.6558 & 0.0608 & 0.3089 & 0.0025 & 0.0891 & 0.0010 & 1.70 & 1788 & 13 & 1759 & 11 & 1735 & 12 & 1725 & 19 & 1788 & 26 \\
\hline OTQ11-031 & 0.0517 & 0.0033 & 0.0575 & 0.0036 & 0.0081 & 0.0001 & 0.0026 & 0.0000 & 0.81 & 273 & 120 & 57 & 3 & 51.8 & 0.6 & 52.1 & 0.8 & 51.8 & 12 \\
\hline OTQ11-032 & 0.0512 & 0.0059 & 0.2160 & 0.0246 & 0.0306 & 0.0006 & 0.0104 & 0.0006 & 2.08 & 250 & 222 & 199 & 21 & 194 & 4 & 209 & 12 & 194 & 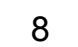 \\
\hline OTQ11-033 & 0.0594 & 0.0009 & 0.7186 & 0.0090 & .0878 & 0.0006 & 0.0271 & 0.0002 & 1.20 & 580 & 15 & 550 & 5 & 543 & 4 & 541 & 4 & 43 & \\
\hline TTQ11-034 & 0.0515 & 0.0020 & 0.1479 & 0.0054 & 0.0209 & 0.0002 & 0.0064 & 0001 & 0.87 & 262 & 66 & 140 & 5 & 133 & 1 & 128 & 2 & 33 & \\
\hline DTQ11 & 0.0558 & 0.0009 & 2657 & .0035 & 0.0345 & 0.0002 & 0.0066 & 0.0001 & 0.97 & 446 & 17 & 239 & 3 & 219 & 1 & 133 & 1 & 219 & \\
\hline TQ11 & 0478 & .0029 & .0916 & .0055 & .0139 & .0002 & 0.0044 & .0001 & 1.20 & 90 & 110 & 89 & 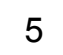 & 89 & 1 & 88 & 2 & 89 & \\
\hline DTQ11-037 & 0633 & 0036 & 9546 & 0525 & 1094 & .0018 & 0.0323 & 06 & 0.45 & 718 & 89 & 680 & 27 & 669 & 10 & 642 & 11 & 669 & \\
\hline JTQ11-038 & 0521 & .0016 & 1005 & .0029 & 0.0140 & 0001 & 0.0044 & 001 & 1.50 & 291 & 51 & 97 & 3 & 89.6 & 0.7 & 90 & 1 & 89.6 & \\
\hline OTQ11-039 & 0521 & 0.0062 & 1045 & .0122 & 0.0145 & 0.0002 & 0.0048 & .0001 & 0.99 & 291 & 237 & 101 & 11 & 93 & 1 & 97 & 3 & 93 & \\
\hline-040 & 0.0777 & 0.0011 & 1.9385 & .0213 & 0.1811 & 0.0013 & 0.0529 & 0.0004 & 1.24 & 1138 & 11 & 1094 & 7 & 1073 & 7 & 1042 & 7 & 1138 & \\
\hline JTQ11-041 & 0.0524 & 0.0026 & 0.1101 & 0.0054 & 0.0153 & 0.0002 & 0.0049 & 0.0001 & 1.46 & 302 & 92 & 106 & 5 & 98 & 1 & 98 & 2 & 98 & \\
\hline OTQ11-042 & 0.0536 & 0.0013 & 0.4291 & 0.0098 & 0.0581 & 0.0005 & 0.0182 & 0.0001 & 5.60 & 354 & 56 & 363 & 7 & 364 & 3 & 364 & 2 & 364 & \\
\hline OTQ11-043 & 0.0499 & 0.0026 & 0.0593 & 0.0029 & 0.0086 & 0.0001 & 0.0027 & 0.0001 & 1.52 & 189 & 93 & 58 & 3 & 55.4 & 0.6 & 54 & 1 & 55.4 & \\
\hline OTQ11-044 & 0.0487 & 0.0025 & 0.1981 & 0.0099 & 0.0295 & 0.0003 & 0.0091 & 0.0002 & 1.15 & 131 & 94 & 184 & 8 & 188 & 2 & 183 & 3 & 188 & \\
\hline 045 & 0.1535 & 0.0017 & 8.9396 & 0.0742 & 0.4226 & 0.0030 & 0.1085 & 0.0007 & 0.98 & 2385 & 6 & 2332 & 8 & 2272 & 14 & 2082 & 13 & 2385 & \\
\hline 10TQ & 0.0566 & 0.0013 & 0.4629 & 0.0098 & 0.0593 & 0.0005 & 0.0186 & 0.0003 & 2.14 & 476 & 33 & 386 & 7 & 372 & 3 & 372 & 5 & 372 & \\
\hline OTQ11-047 & 0.0514 & 0.0029 & 0.1142 & 0.0062 & 0.0161 & 0.0002 & 0.0052 & 0.0001 & 1.18 & 257 & 105 & 110 & 6 & 103 & 1 & 104 & 2 & 103 & \\
\hline-048 & 0.0515 & 0.0041 & 0.0609 & .0047 & 0.0086 & 0.0001 & 0.0028 & 0.0001 & 1.12 & 265 & 152 & 60 & 5 & 55 & 0.8 & 56 & 1 & 55 & 0 \\
\hline OTQ11-049 & 0.0503 & 0.0032 & 0.1015 & 0.0062 & 0.0146 & 0.0002 & 0.0048 & 0.0001 & 1.09 & 208 & 117 & 98 & 6 & 94 & 1 & 96 & 2 & 94 & \\
\hline $1-050$ & 0.0509 & 0.0039 & 0.1103 & 0083 & 0.0157 & 0.0002 & 0.0055 & 0.0002 & 1.27 & 236 & 142 & 106 & 8 & 101 & 2 & 110 & 3 & 101 & \\
\hline OTQ11-051 & 0.0504 & 0.0032 & 0.1059 & .0066 & 0.0152 & 0.0002 & 0.0046 & 0.0001 & 1.16 & 214 & 120 & 102 & 6 & 98 & 1 & 93 & 2 & 98 & \\
\hline $1-052$ & 0.0530 & 0.0037 & 0.2161 & 0.0146 & 0.0296 & 0.0004 & 0.0092 & 0.0 & 1.97 & 328 & 130 & 199 & 12 & 188 & 3 & 185 & 7 & 88 & \\
\hline OTC & 0.1182 & 0.0016 & 5.4 & 0.0604 & 0.3 & 0.0026 & 0.0 & 0.0 & 2.63 & 1929 & 10 & 1888 & 10 & 1852 & 12 & 1723 & 20 & 1929 & 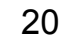 \\
\hline 10TC & 0.0512 & 0.00 & 0.0563 & 1 & .0080 & 01 & 0.0024 & 00 & 0.86 & 2 & 32 & 56 & 1 & 51.2 & 0.4 & 49.3 & 0.4 & 51.2 & \\
\hline 55 & 0.0615 & 0.0009 & 8116 & .0106 & .0957 & 07 & 0.0288 & 02 & 0.35 & 65 & 16 & 603 & 6 & 589 & 4 & 573 & 3 & 589 & \\
\hline 056 & 0.0495 & 0.0082 & 0.0758 & .0123 & 0.0111 & 0.0003 & 0.0037 & 0.0002 & 0.88 & 173 & 282 & 74 & 12 & 71 & 2 & 75 & 3 & 71 & \\
\hline 057 & 0.0509 & 0.0144 & .0631 & .0178 & .0090 & 0.0002 & 0.0027 & 0.0001 & 0.73 & 236 & 467 & 62 & 17 & 58 & 1 & 54 & 3 & 58 & \\
\hline OTQ11-058 & 0.0867 & 0.0014 & 2.7324 & .0402 & 0.2286 & 0.0019 & 0.0654 & 0.0006 & 1.01 & 1354 & 16 & 1337 & 11 & 1327 & 10 & 1280 & 12 & 1354 & \\
\hline-059 & 0.0485 & 0.0041 & 1337 & .0113 & 0.0200 & 0.0003 & 0.0061 & 0.0001 & 0.81 & 123 & 161 & 127 & 10 & 128 & 2 & 123 & 3 & 128 & \\
\hline OTQ11-060 & 0.0491 & 0.0019 & 0.0972 & 0.0036 & 0.0144 & 0.0001 & 0.0049 & 0.0001 & 4.24 & 15 & 68 & 94 & 3 & 91.9 & 0.9 & 98 & 3 & 91.9 & \\
\hline TQ11-061 & 0.0596 & 0.0007 & 0.8100 & .0069 & 0.0987 & 0.0007 & 0.0287 & 0.0004 & 25.32 & 588 & 9 & 602 & 4 & 607 & 4 & 572 & 8 & 607 & \\
\hline JTQ11-062 & 0.0960 & 0.0011 & 3.7783 & 0.0321 & 0.2854 & 0.0020 & 0.0790 & 0.0006 & 2.62 & 1548 & 7 & 1588 & 7 & 1619 & 10 & 1536 & 11 & 1548 & \\
\hline
\end{tabular}




\begin{tabular}{|c|c|c|c|c|c|c|c|c|c|c|c|c|c|c|c|c|c|c|c|}
\hline 10TQ11-063 & .0491 & 0.0034 & 0.0944 & 0.0064 & 0.0139 & 0.0002 & 0.0042 & 0.0001 & 1.04 & 153 & 128 & 92 & 6 & 89 & 1 & 85 & 2 & 89 & 2 \\
\hline 0TQ11-064 & .0536 & 0.0010 & 4412 & 0.0070 & 0.0597 & 0.0004 & 0.0190 & 0.0002 & 1.63 & 355 & 23 & 371 & 5 & 374 & 3 & 379 & 3 & 374 & 6 \\
\hline 0TQ11-065 & 0.0496 & 0.0035 & 0.0908 & 0.0064 & 0.0133 & 0.0002 & 0.0043 & 0.0001 & 1.15 & 178 & 134 & 88 & 0 & 85 & 1 & 87 & 2 & 85 & 2 \\
\hline 0TQ11-066 & 0.0479 & 0.0018 & 0.0863 & 0.0031 & 0.0131 & 0.0001 & 0.0042 & 0.0000 & 2.16 & 94 & 82 & 84 & 0 & 83.7 & 0.7 & 83.7 & 0.6 & 83.7 & 1.4 \\
\hline OTQ11-067 & 0.1132 & 0.0013 & 1276 & 0.0454 & 0.3287 & 0.0023 & 0.0923 & 0.0009 & 3.43 & 1851 & 7 & 1841 & 0 & 1832 & 11 & 1785 & 16 & 1851 & 11 \\
\hline JTQ11-068 & 0.0603 & 0.0010 & 0.8263 & 0.0117 & 0.0994 & 0.0007 & 0.0293 & 0.0002 & 0.89 & 615 & 18 & 612 & 7 & 611 & 4 & 584 & 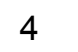 & 611 & 8 \\
\hline DTQ11-069 & 0.0503 & .0035 & .0565 & 0.0039 & 0.0082 & 0.0001 & 0.0027 & 0.0001 & 3.02 & 208 & 130 & 56 & 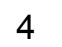 & 52.4 & 0.8 & 55 & 2 & 52.4 & 1.6 \\
\hline OTQ11-070 & 0.0480 & 0032 & 0.0922 & 0.0060 & 0.0139 & 0.0002 & 0.0041 & 0.0001 & 0.95 & 98 & 122 & 90 & 6 & 89 & 1 & 83 & 2 & 89 & \\
\hline 1 & 0.0 & 0.003 & 0.2 & 0.0133 & 38 & 0.0004 & 33 & 2 & .28 & 284 & 126 & 191 & 11 & 183 & 2 & 187 & 4 & 183 & \\
\hline 72 & 0.0774 & 0.0032 & 0 & 0.0832 & 1915 & 0.0026 & 72 & 2 & .96 & 1132 & 59 & 130 & 8 & 1130 & 14 & 124 & 22 & 1132 & \\
\hline 73 & $0 / 91$ & 15 & 2691 & 0400 & 2083 & 0018 & 88 & 04 & .28 & 173 & 21 & 203 & 2 & 1219 & 10 & 155 & & 1173 & \\
\hline 74 & 0747 & 0019 & 8183 & .0431 & 1767 & 0017 & 11 & 07 & .10 & 1059 & 32 & 052 & 6 & 1049 & & 007 & 3 & 1059 & \\
\hline 075 & 0.0524 & 0061 & 1318 & 0.0150 & .0183 & .0004 & 0.0090 & .0003 & 1.27 & 301 & 214 & 126 & 3 & 117 & & 181 & & 117 & \\
\hline-076 & 0.0506 & .0045 & .0442 & 0.0039 & .0063 & 0.0001 & 0.0021 & 0.0001 & 0.63 & 224 & 165 & 44 & & 40.7 & 0.7 & 42 & 1 & 40.7 & 4 \\
\hline 077 & 0.0501 & .0010 & 1088 & 0.0020 & .0157 & .0001 & 0.0048 & .0000 & 1.00 & 201 & 29 & 10 & 2 & 100.7 & 0.8 & 96.2 & 0.8 & 100.7 & \\
\hline OTQ11-078 & 0.0589 & 0.0013 & 0.8673 & 0.0182 & 0.1068 & .0009 & 0.0273 & 0.0004 & 1.56 & 563 & 31 & 634 & 10 & 654 & 5 & 544 & 7 & 654 & 0 \\
\hline 079 & 0.0524 & 0.0028 & 1198 & 0.0063 & 0.0166 & 0.0002 & 0.0049 & 0.0001 & 0.66 & 301 & 101 & 115 & 0 & 106 & 1 & 98 & 1 & 106 & \\
\hline 080 & 0.0489 & 0.0016 & 0.0950 & 0.0030 & 0.0141 & 0.0001 & 0.0042 & 0.0000 & 0.70 & 144 & 56 & 92 & 3 & 90.2 & 0.8 & 84.9 & 0.8 & 90.2 & 6 \\
\hline 081 & 0.0516 & .0045 & .0984 & 0.0084 & 0.0138 & 0.0002 & 0.0 & 0.0001 & 0.81 & 267 & 170 & 95 & & $8 s$ & 1 & 85 & ? & 89 & \\
\hline 82 & 0.0472 & 0.0026 & 0.0486 & 0.0026 & 0.0075 & 0.0001 & 0.0026 & 0.0001 & 3.06 & 60 & 96 & 48 & 0 & 48 & 0.6 & 53 & 2 & 48 & 1.2 \\
\hline 10TQ & 0.0519 & 0.0041 & 0.1133 & 0.0088 & 0.0158 & 0.0002 & 0.0049 & 0.0001 & 1.01 & 283 & 150 & 109 & 8 & 101 & 1 & 99 & 3 & 101 & 2 \\
\hline 84 & 0.0492 & 0.0264 & .0561 & 0.0298 & 0.0083 & 0.0007 & 0.0030 & 0.0004 & 0.80 & 158 & 807 & 55 & 29 & 53 & 4 & 61 & 8 & 53 & 8 \\
\hline 85 & 0.0482 & 0.0028 & 0.0548 & 0.0031 & 0.0083 & 0.0001 & 0.0026 & 0.0000 & 0.98 & 108 & 129 & 54 & 3 & 53 & 0.6 & 52.8 & 0.4 & 53 & 1.2 \\
\hline 86 & 0.0533 & .0013 & & 0.0073 & 0.0429 & 0.0004 & & 0.0001 & 0.81 & 342 & 37 & 278 & 6 & 271 & $?$ & 259 & 2 & 71 & 4 \\
\hline 10TC & 0.0 & 1 & & 0.0284 & 0.0291 & 0.0006 & & & 1.63 & 64 & 261 & 176 & 24 & 185 & 4 & 190 & 1 & 85 & 8 \\
\hline 10TC & 0.0 & & & 0.0379 & & & & & 0.97 & 1220 & 19 & 1224 & 12 & 1227 & 9 & 1176 & 11 & 1220 & 38 \\
\hline 10TC & & 1 & & 0.0016 & 0.0 & & & & 1.03 & 121 & 78 & 37 & ? & 35.9 & .4 & 32.9 & 0.6 & 35.9 & .8 \\
\hline 10TC & & & & 0.0035 & & & & & 1.24 & 104 & 71 & 89 & 3 & 88.6 & 0.8 & 81 & 1 & 6 & 6 \\
\hline 91 & 6 & 2 & & 0.0223 & 0.1532 & 12 & & & 2.09 & 85 & 2( & & 0 & 91 & 7 & 42 & 0 & 919 & \\
\hline-092 & 0.0783 & 0019 & & 0.0512 & 2078 & 0.0020 & 0.0596 & 0.0007 & 0.71 & 1156 & 30 & 1195 & 16 & 1217 & 11 & 1171 & 13 & 1156 & 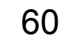 \\
\hline 93 & 0.0503 & 0019 & & 0.0037 & 0.0151 & 0.0001 & 0.0042 & 0.0001 & 0.77 & $20 \varepsilon$ & 65 & 10 & 3 & 96.4 & 0.9 & 85 & 1 & 96.4 & \\
\hline & & & & 0.0012 & & 001 & & 0.0000 & 2.20 & 18 & 36 & 5 & 1 & 50 & 0.4 & 47 & 0.6 & 50 & \\
\hline & 0.0499 & 0032 & & 0.0046 & 0.0107 & 0.0001 & 0.0032 & 0.0001 & 1.00 & 188 & 119 & 72 & 4 & 68.6 & 0.8 & 65 & 1 & 68.6 & 6 \\
\hline 10TQ11-096 & 0.0523 & 0.0018 & 0.2265 & 0.0076 & 0.0314 & 0.0003 & 0.0088 & 0.0002 & 1.60 & 299 & 59 & 207 & 6 & 199 & 2 & 178 & 3 & 199 & \\
\hline
\end{tabular}




\begin{tabular}{|c|c|c|c|c|c|c|c|c|c|c|c|c|c|c|c|c|c|c|c|}
\hline 10TQ11-097 & 0.0520 & 0.0052 & 0.1502 & 0.0148 & 0.0210 & 0.0003 & 0.0063 & 0.0002 & 1.04 & 283 & 193 & 142 & 13 & 134 & 2 & 127 & 4 & 134 & 4 \\
\hline 10TQ11-098 & 0.0490 & 0.0036 & 0.1141 & 0.0081 & 0.0169 & 0.0002 & 0.0047 & 0.0001 & 0.94 & 148 & 134 & 110 & 7 & 108 & 2 & 95 & 2 & 108 & 4 \\
\hline 10TQ11-099 & 0.0485 & 0.0178 & 0.0618 & 0.0225 & 0.0093 & 0.0005 & 0.0034 & 0.0003 & 1.04 & 122 & 533 & 61 & 22 & 59 & 3 & 69 & 7 & 59 & 6 \\
\hline 10TQ11-100 & 0.0524 & 0.0045 & 0.1346 & 0.0114 & 0.0186 & 0.0003 & 0.0054 & 0.0002 & 0.94 & 302 & 161 & 128 & 10 & 119 & 2 & 109 & 3 & 119 & 4 \\
\hline 10TQ11-101 & 0.0508 & 0.0046 & 0.0570 & 0.0051 & 0.0081 & 0.0002 & 0.0025 & 0.0001 & 0.64 & 230 & 167 & 56 & 5 & 52.3 & 1 & 51 & 1 & 52.3 & 2 \\
\hline 10TQ11-102 & 0.1187 & 0.0014 & 5.1969 & 0.0497 & 0.3176 & 0.0023 & 0.1029 & 0.0007 & 0.99 & 1937 & 8 & 1852 & 8 & 1778 & 11 & 1980 & 13 & 1937 & 16 \\
\hline 10TQ11-103 & 0.0968 & 0.0016 & 3.7655 & 0.0539 & 0.2821 & 0.0024 & 0.0756 & 0.0010 & 2.01 & 1564 & 15 & 1585 & 11 & 1602 & 12 & 1473 & 19 & 1564 & 30 \\
\hline 10TQ11-104 & 0.0528 & 0.0016 & 0.2295 & 0.0064 & 0.0316 & 0.0003 & 0.0093 & 0.0001 & 0.84 & 318 & 47 & 210 & 5 & 200 & 2 & 187 & 2 & 200 & 4 \\
\hline 10TQ11-105 & 0.0506 & 0.0031 & 0.0980 & 0.0058 & 0.0141 & 0.0002 & 0.0043 & 0.0001 & 0.98 & 222 & 112 & 95 & 5 & 90 & 1 & 86 & 2 & 90 & 2 \\
\hline
\end{tabular}

2010TQ14 (Dazuqu, Qiabulin section, n=104, N29 $19.253^{\prime}$, E88 ${ }^{\circ} 31.332^{\prime}, 4075 \mathrm{~m}$ )

$\begin{array}{llllllllll}\text { 10TQ14-001 } & 0.0765 & 0.0017 & 1.9439 & 0.0409 & 0.1843 & 0.0017 & 0.0524 & 0.0004 & 0.32\end{array}$

$\begin{array}{llllllllllll}1074-002 & 0.0466 & 0.0012 & 0.0374 & 0.0009 & 0.0058 & 0.0001 & 0.0019 & 0.0000 & 2.20\end{array}$

$\begin{array}{llllllllll}\text { 10TQ14-004 } & 0.0490 & 0.0121 & 0.0521 & 0.0127 & 0.0077 & 0.0002 & 0.0026 & 0.0001 & 0.88\end{array}$

$\begin{array}{llllllllll}10 T Q 14-005 & 0.0515 & 0.0053 & 0.0587 & 0.0059 & 0.0083 & 0.0002 & 0.0025 & 0.0001 & 0.89\end{array}$

$\begin{array}{lllllllllll}10 T Q 14-006 & 0.0510 & 0.0182 & 0.0616 & 0.0218 & 0.0088 & 0.0004 & 0.0032 & 0.0003 & 0.93\end{array}$

10TQ14-007 $0.0487 \quad 0.0101$

10TQ14-008 $0.1130 \quad 0.0012$

10TQ14-009 $0.0531 \quad 0.0107$

$\begin{array}{lll}10 T Q 14-010 & 0.0477 & 0.0123\end{array}$

$\begin{array}{lll}10 T Q 14-011 & 0.0518 & 0.0033\end{array}$

10TQ14-012 $0.0496 \quad 0.0026$

10TQ14-013 $0.0480 \quad 0.0025$

10TQ14-014 $0.0769 \quad 0.0014$

10TQ14-015 $0.0507 \quad 0.0028$

10TQ14-016 $0.0497 \quad 0.0055$

$\begin{array}{lll}10 T Q 14-017 & 0.0502 & 0.0018\end{array}$

$\begin{array}{lll}10 T Q 14-018 & 0.0511 & 0.0018\end{array}$

$\begin{array}{lll}10 T Q 14-019 & 0.0768 & 0.0018\end{array}$

10TQ14-020 $0.0498 \quad 0.0075$

$\begin{array}{lll}10 T Q 14-021 & 0.0503 & 0.0038\end{array}$

$\begin{array}{lll}10 T Q 14-022 & 0.0492 & 0.0197\end{array}$

10TQ14-023 $0.0507 \quad 0.0048$ $\begin{array}{llllllll}0.1003 & 0.0205 & 0.0150 & 0.0005 & 0.0051 & 0.0003 & 1.17\end{array}$

$\begin{array}{llllllll}5.4334 & 0.0399 & 0.3490 & 0.0023 & 0.1031 & 0.0008 & 5.64\end{array}$

$\begin{array}{lllllll}0.1229 & 0.0246 & 0.0168 & 0.0004 & 0.0053 & 0.0003 & 0.94\end{array}$

$\begin{array}{llllllll}0.0601 & 0.0153 & 0.0092 & 0.0004 & 0.0045 & 0.0004 & 2.25\end{array}$

$\begin{array}{llllllll}0.1083 & 0.0056 & 0.0159 & 0.0002 & 0.0049 & 0.0001 & 1.41\end{array}$

$\begin{array}{llllllll}0.0939 & 0.0048 & 0.0142 & 0.0002 & 0.0042 & 0.0001 & 0.95\end{array}$

$\begin{array}{lllllll}2.0335 & 0.0323 & 0.1918 & 0.0016 & 0.0583 & 0.0007 & 1.67\end{array}$

$\begin{array}{llllllll}0.0771 & 0.0042 & 0.0110 & 0.0001 & 0.0035 & 0.0001 & 0.87\end{array}$

$\begin{array}{llllllll}0.0587 & 0.0064 & 0.0086 & 0.0002 & 0.0025 & 0.0001 & 0.48\end{array}$

$\begin{array}{llllllll}0.2142 & 0.0074 & 0.0309 & 0.0003 & 0.0098 & 0.0001 & 2.35\end{array}$

$\begin{array}{llllllll}0.0498 & 0.0017 & 0.0071 & 0.0001 & 0.0021 & 0.0000 & 0.52\end{array}$

$\begin{array}{llllllll}1.9644 & 0.0421 & 0.1857 & 0.0017 & 0.0562 & 0.0006 & 0.66\end{array}$

$\begin{array}{lllllll}0.0703 & 0.0105 & 0.0102 & 0.0002 & 0.0033 & 0.0002 & 1.66\end{array}$

$\begin{array}{llllllll}0.0527 & 0.0039 & 0.0076 & 0.0001 & 0.0022 & 0.0001 & 2.21\end{array}$

$\begin{array}{lllllll}0.0571 & 0.0228 & 0.0084 & 0.0003 & 0.0044 & 0.0003 & 1.69\end{array}$

$\begin{array}{llllllll}0.0987 & 0.0091 & 0.0141 & 0.0003 & 0.0042 & 0.0001 & 0.81\end{array}$ $\begin{array}{lllllll}0.1059 & 0.0066 & 0.0148 & 0.0002 & 0.0047 & 0.0001 & 1.46\end{array}$ $\begin{array}{llllllllll}1108 & 27 & 1096 & 14 & 1091 & 9 & 1031 & 8 & 1091 & 18\end{array}$

$\begin{array}{llllllllll}30 & 38 & 37.3 & 0.9 & 37.5 & 0.3 & 37.6 & 0.6 & 37.5 & 0.6\end{array}$

$\begin{array}{llllllllll}183 & 231 & 58 & 7 & 55 & 1 & 54 & 2 & 55 & 2\end{array}$

$\begin{array}{llllllllll}148 & 392 & 52 & 12 & 50 & 1 & 52 & 2 & 50 & 2\end{array}$

$\begin{array}{llllllllll}264 & 195 & 58 & 6 & 53 & 1 & 50 & 2 & 53 & 2\end{array}$

$\begin{array}{llllllllll}242 & 551 & 61 & 21 & 56 & 2 & 64 & 5 & 56 & 4\end{array}$

$\begin{array}{llllllllll}131 & 325 & 97 & 19 & 96 & 3 & 102 & 7 & 96 & 6\end{array}$

$\begin{array}{llllllllll}1847 & 6 & 1890 & 6 & 1930 & 11 & 1984 & 15 & 1847 & 12\end{array}$

$\begin{array}{llllllllll}334 & 371 & 118 & 22 & 107 & 2 & 108 & 6 & 107 & 4\end{array}$

$\begin{array}{llllllllll}82 & 378 & 59 & 15 & 59 & 2 & 91 & 8 & 59 & 4\end{array}$

$\begin{array}{llllllllll}277 & 117 & 102 & 6 & 95 & 1 & 94 & 3 & 95 & 2\end{array}$

$\begin{array}{llllllllll}175 & 95 & 104 & 5 & 101 & 1 & 99 & 2 & 101 & 2\end{array}$

$\begin{array}{llllllllll}101 & 93 & 91 & 4 & 91 & 1 & 85 & 2 & 91 & 2\end{array}$

$\begin{array}{llllllllll}1119 & 19 & 1127 & 11 & 1131 & 8 & 1145 & 12 & 1119 & 38\end{array}$

$\begin{array}{lllllllllll}225 & 104 & 75 & 4 & 70.8 & 0.8 & 70 & 1 & 70.8 & 1.6\end{array}$

$\begin{array}{llllllllll}181 & 209 & 58 & 6 & 55 & 1 & 51 & 1 & 55 & 2\end{array}$

$\begin{array}{llllllllll}205 & 85 & 197 & 6 & 196 & 2 & 196 & 1 & 196 & 4\end{array}$

$\begin{array}{llllllllll}243 & 59 & 49 & 2 & 45.4 & 0.4 & 42.2 & 0.4 & 45.4 & 0.8\end{array}$

$\begin{array}{llllllllll}1115 & 28 & 1103 & 14 & 1098 & 9 & 1106 & 11 & 1115 & 56\end{array}$

$\begin{array}{llllllllll}187 & 277 & 69 & 10 & 66 & 1 & 66 & 3 & 66 & 2\end{array}$

$\begin{array}{llllllllll}209 & 137 & 52 & 4 & 48.9 & 0.8 & 45 & 2 & 48.9 & 1.6\end{array}$

$\begin{array}{llllllllll}159 & 623 & 56 & 22 & 54 & 2 & 88 & 7 & 54 & 4\end{array}$

$\begin{array}{lccccccccc}226 & 173 & 96 & 8 & 90 & 2 & 84 & 3 & 90 & 4\end{array}$ 


\begin{tabular}{|c|c|c|c|c|c|c|c|c|c|c|c|c|c|c|c|c|c|c|c|}
\hline 0TQ14-024 & .0524 & 0.0245 & 0.1177 & 0.0546 & 0.0163 & 0.0009 & 0.0054 & 0.0008 & 1.20 & 305 & 744 & 113 & 50 & 104 & 6 & 109 & 15 & 104 & 12 \\
\hline 10TQ14-025 & 0.0509 & 0.0078 & 0.0584 & 0.0089 & 0.0083 & 0.0002 & 0.0029 & 0.0002 & 1.76 & 238 & 282 & 58 & 8 & 53 & 1 & 59 & 3 & 53 & 2 \\
\hline OTQ14-026 & 0.0501 & 0.0033 & 0.0993 & 0.0063 & 0.0144 & 0.0002 & 0.0048 & 0.0001 & 1.23 & 201 & 119 & 96 & 6 & 92 & 1 & 96 & 2 & 92 & 2 \\
\hline 0TQ14-027 & 0.1034 & 0.0016 & 3.9981 & 0.0541 & 0.2806 & 0.0023 & 0.0785 & 0.0006 & 0.64 & 1685 & 14 & 1634 & 11 & 1594 & 11 & 1527 & 12 & 1685 & 28 \\
\hline OTQ14-028 & 0.0483 & 0.0024 & 0.0459 & 0.0022 & 0.0069 & 0.0001 & 0.0023 & 0.0000 & 0.80 & 112 & 87 & 46 & 2 & 44.4 & 0.5 & 46 & 0.6 & 44.4 & 1 \\
\hline 0TQ14-029 & 0.0502 & 0.0097 & 0.0517 & 0.0099 & 0.0075 & 0.0002 & 0.0024 & 0.0001 & 0.85 & 205 & 358 & 51 & 10 & 48 & 1 & 48 & 2 & 48 & \\
\hline OTQ14-030 & 0.0797 & 8 & 2.1708 & 0.0449 & 0.1977 & 0.0019 & 0.0604 & 0.0008 & 15 & 188 & 26 & 1172 & 14 & 1163 & 10 & 1185 & 15 & 188 & \\
\hline DTQ14-031 & 0.0837 & 0019 & 5597 & 0.0539 & 0.2219 & .0018 & 0.0660 & .0005 & 1.28 & 285 & 45 & 1289 & 15 & 1292 & 10 & 1292 & & 1285 & \\
\hline TQ14-032 & 0.0528 & 0073 & 1762 & .0239 & 0242 & 0007 & 78 & 04 & .08 & 318 & 253 & 165 & 21 & 154 & 4 & 157 & 0 & 154 & \\
\hline JTQ14-033 & 0.0483 & 0013 & 0.0364 & 0.0009 & .0055 & .0000 & 0.0018 & 000 & 1.58 & 113 & 45 & 36.3 & 0.9 & 35.2 & 0.3 & 35.7 & 0.4 & 35.2 & \\
\hline OTQ14-034 & 0.0517 & .0041 & 0.0923 & 0.0071 & 0.0130 & 0.0002 & 0.0042 & .0001 & 0.89 & 271 & 146 & 90 & 7 & 83 & 1 & 86 & 2 & 83 & \\
\hline OTQ14-035 & 0.0955 & 0.0013 & 3.5727 & 0.0399 & 0.2713 & 0.0019 & 0.0796 & 0.0006 & 4.49 & 1538 & 25 & 1543 & 9 & 1547 & 10 & 1548 & 11 & 1547 & \\
\hline JTQ14-036 & 0.0516 & 0.0085 & 0.0561 & 0.0091 & 0.0079 & 0.0002 & 0.0028 & 0.0001 & 0.81 & 266 & 293 & 55 & 9 & 51 & 1 & 56 & 2 & 51 & \\
\hline 0TQ14-037 & 0.0487 & 0.0092 & 0.0484 & 0.0090 & 0.0072 & 0.0002 & 0.0033 & 0.0002 & 1.88 & 134 & 299 & 48 & 9 & 46 & 1 & 66 & 4 & 46 & \\
\hline OTQ14-038 & 0.0495 & 0.0037 & 0.0561 & 0.0041 & 0.0082 & 0.0001 & 0.0026 & 0.0001 & 0.92 & 169 & 138 & 55 & 4 & 52.8 & 0.8 & 52 & 1 & 52.8 & \\
\hline OTQ14-039 & 0.0515 & 0.0032 & 0.0575 & 0.0035 & 0.0081 & 0.0001 & 0.0031 & 0.0001 & 1.89 & 265 & 117 & 57 & 3 & 52 & 0.6 & 63 & 2 & 52 & \\
\hline DTQ14-040 & 0.0609 & 0.0015 & 0.6814 & 0.0155 & 0.0812 & 0.0007 & 0.0251 & 0.0003 & 0.84 & 635 & 34 & 528 & 0 & 503 & 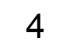 & 501 & 5 & 503 & \\
\hline OTQ14-041 & 0.0815 & 0.0011 & 2.4398 & 0.0267 & 0.2172 & 0.0016 & 0.0648 & 0.0005 & 1.71 & 1233 & 11 & 1254 & 8 & 1267 & 8 & 1269 & 10 & 1233 & \\
\hline 10TQ14-042 & 0.0474 & 0.0021 & 0.0972 & 0.0042 & 0.0149 & 0.0002 & 0.0044 & 0.0001 & 0.77 & 67 & 76 & 94 & 4 & 95.3 & 1 & 88 & 1 & 95.3 & \\
\hline 10TQ14-043 & 0.0515 & 0.0045 & 0.0509 & 0.0043 & 0.0072 & 0.0001 & 0.0026 & 0.0001 & 1.32 & 265 & 160 & 50 & 4 & 46.1 & 0.8 & 53 & 2 & 46.1 & 6 \\
\hline OTQ14-044 & 0.0461 & 0.0011 & 0.0341 & 0.0008 & 0.0054 & 0.0000 & 0.0017 & 0.0000 & 2.83 & 1 & 47 & 34 & 0.7 & 34.5 & 0.2 & 34.6 & 0.6 & 34.5 & 4 \\
\hline OTQ14-045 & 0.0473 & .0071 & 0.0546 & 0.0082 & 0.0084 & 0.0002 & 0.0030 & 01 & 1.46 & 64 & 265 & 54 & 8 & 54 & 1 & 60 & 2 & 54 & \\
\hline 10TQ14-046 & 0.0485 & 0.00 & 0.0357 & 0.0071 & 0.0053 & 0.0001 & 0.0017 & 01 & 1.02 & 121 & 357 & 36 & 7 & 34.4 & 0.7 & 34 & 2 & 34.4 & 4 \\
\hline 10TQ14-047 & 0.0505 & 0.0043 & 0.0964 & 0.0080 & 0.0139 & 0.0002 & 0.0043 & 01 & 1.16 & 216 & 159 & 93 & 7 & 89 & 1 & 86 & 3 & 89 & \\
\hline 10TQ14-048 & 0.0 & 0.00 & 0.1 & 0.0226 & 0.02 & 0.0 & 0.0 & & 1.06 & 322 & 234 & 168 & 19 & 158 & 4 & 167 & 8 & 158 & \\
\hline 10TQ14-C & 0.0 & 0 & & 0.07 & 0.0 & 88 & & & 2.10 & 265 & 599 & 183 & 62 & 17 & 5 & 286 & 16 & 177 & 0 \\
\hline 10TQ14-050 & 0.0688 & 0014 & 38 & 0.0263 & 1437 & 0.0011 & 0.0437 & & 1.58 & 894 & 44 & 874 & 11 & 866 & 0 & 864 & 5 & 866 & \\
\hline 0TQ14-051 & 0.0508 & .0037 & 0.0544 & 0.0038 & 0.0078 & 0.0001 & 0.0023 & 0.0000 & 0.40 & 230 & 131 & 5 & 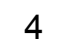 & 50 & 0.8 & 45.8 & 0.8 & 50 & \\
\hline 0TQ14-052 & 0.0521 & .0037 & 1042 & 0.0073 & 0.0145 & 0.0002 & 0.0047 & 0.0002 & 2.21 & 290 & 138 & 10 & $r$ & 93 & 1 & 95 & 3 & 93 & \\
\hline 0TQ14-053 & 0.0491 & .0064 & 0.0582 & 0.0075 & 0.0086 & 0.0002 & 0.0028 & 0.0001 & 1.45 & 152 & 248 & 57 & 7 & 55 & 1 & 56 & 3 & 55 & \\
\hline OTQ14-054 & 0.0503 & .0055 & 0.0971 & 0.0106 & 0.0140 & 0.0002 & 0.0052 & 0.0002 & 2.23 & 207 & 221 & 94 & 10 & 90 & 1 & 105 & 4 & 90 & \\
\hline 0TQ14-055 & 0.0849 & 0.0013 & 2.2036 & 0.0279 & 0.1883 & 0.0014 & 0.0636 & 0.0006 & 1.43 & 1313 & 13 & 1182 & 9 & 1112 & 8 & 1246 & 10 & 1313 & \\
\hline 0TQ14-056 & 0.0507 & .0020 & 0.0859 & 0.0033 & 0.0123 & 0.0001 & 0.0038 & 0.0001 & 0.60 & 227 & 68 & 84 & 3 & 78.7 & 0.8 & 76 & 1 & 78.7 & \\
\hline OTQ14-057 & 0.0526 & 0.0083 & 0.2238 & 0.0351 & 0.0309 & 0.0006 & 0.0106 & 0.0005 & 1.18 & 312 & 304 & 205 & 29 & 196 & 4 & 212 & 9 & 196 & \\
\hline
\end{tabular}




\begin{tabular}{|c|c|c|c|c|c|c|c|c|c|c|c|c|c|c|c|c|c|c|c|}
\hline 10TQ14-058 & .0517 & 0.0139 & 0.0570 & 0.0153 & 0.0080 & 0.0002 & 0.0030 & 0.0002 & 1.08 & 270 & 454 & 56 & 15 & 51 & 1 & 60 & 3 & 51 & 2 \\
\hline 0TQ14-059 & .0542 & 0.0008 & .4273 & 0.0049 & 0.0572 & 0.0004 & 0.0179 & 0.0002 & 9.10 & 378 & 14 & 361 & 0 & 359 & 2 & 359 & 5 & 359 & 4 \\
\hline 0TQ14-060 & 0.0461 & 0.0030 & 0.0479 & 0.0031 & 0.0075 & 0.0001 & 0.0028 & 0.0003 & 1.46 & & 144 & 47 & 3 & 48.4 & 0.8 & 57 & 5 & 8.4 & 1.6 \\
\hline 0TQ14-061 & 0.0493 & 0.0014 & 0.0591 & 0.0016 & 0.0087 & 0.0001 & 0.0027 & 0.0001 & 3.07 & 160 & 49 & 58 & 2 & 55.9 & 0.4 & 54 & 1 & 55.9 & 0.8 \\
\hline 0TQ14-062 & 0.0795 & 0.0013 & 2.1619 & 0.0317 & 0.1974 & 0.0016 & 0.0577 & 0.0005 & 1.16 & 1184 & 17 & 1169 & 10 & 1161 & 8 & 1133 & 10 & 1184 & 4 \\
\hline 0TQ14-063 & 0.0511 & 0.0015 & 0.0633 & 0.0017 & 0.0090 & 0.0001 & 0.0029 & 0.0001 & 2.25 & 246 & 48 & 62 & 2 & 57.7 & 0.4 & 59 & 1 & 57.7 & .8 \\
\hline DTQ14-064 & 0.0461 & .0058 & 0.0473 & 0.0059 & 0.0075 & 0.0002 & 0.0025 & 0.0003 & 2.92 & & 242 & 47 & 6 & 48 & 1 & 50 & 0 & 48 & 0 \\
\hline OTQ14-065 & 0.0513 & 0188 & 0.0735 & 0.0265 & 0.0104 & .0006 & 0.0049 & 0.0006 & 1.37 & 253 & 537 & 72 & 25 & 67 & 4 & 99 & 11 & 67 & \\
\hline 6 & 0.0 & 0.00 & 991 & 0.0085 & .0084 & .0002 & .0028 & 0002 & 1.47 & 231 & 277 & 58 & 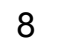 & 54 & 1 & 56 & & 54 & \\
\hline OTC & 0.0 & .0054 & 16 & .0096 & .0130 & .0002 & 41 & 0001 & 0.88 & 241 & 212 & 8 & & 84 & 1 & 82 & & 84 & \\
\hline 38 & 99 & 1 & 30 & .0064 & 0092 & 0001 & 31 & 01 & 1.61 & 188 & 201 & 6. & & 58.8 & 0.9 & 63 & & 3.8 & 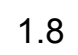 \\
\hline 69 & 96 & 0029 & 71 & .0049 & .0127 & 0002 & 38 & 01 & 0.60 & 176 & 107 & 85 & & 5 & 1 & 76 & 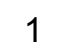 & 1.5 & \\
\hline 70 & 0.0507 & .0023 & 0569 & 0.0025 & .0081 & .0001 & 0.0025 & 0.0000 & 0.63 & 229 & 85 & 5 & & 52.2 & 0.5 & 50.3 & 0.6 & 52.2 & \\
\hline 14-071 & 0.0494 & .0063 & 0922 & 0.0116 & 0.0136 & 0.0003 & 0.0042 & 0.0002 & 1.15 & 166 & 239 & 90 & 11 & 87 & ? & 84 & 4 & 87 & \\
\hline 072 & 0.0492 & .0063 & .0530 & 0.0067 & .0078 & 0.0002 & 0.0025 & 0.0001 & 1.06 & 159 & 267 & 52 & 6 & 50.1 & 1 & 50 & 1 & 50.1 & \\
\hline OTQ14-073 & 0.0508 & 0.0082 & 0.0903 & 0.0145 & 0.0129 & 0.0003 & 0.0051 & 0.0003 & 2.11 & 230 & 296 & 88 & 14 & 83 & 2 & 103 & 6 & 83 & \\
\hline-074 & 0.1013 & 0.0016 & .7723 & 0.0507 & 0.2702 & 0.0021 & 0.0788 & 0.0006 & 6.36 & 1647 & 29 & 1587 & 11 & 1542 & 11 & 1533 & 11 & 1542 & 22 \\
\hline 075 & 0.0515 & 0.0029 & 0.0556 & 0.0030 & 0.0078 & 0.0001 & 0.0027 & 0.0001 & 1.23 & 265 & 104 & 55 & 3 & 50.2 & 0.6 & 53 & 1 & 50.2 & 1.2 \\
\hline $10 \mathrm{TC}$ & 0.0477 & .01 & .0504 & 0.0160 & 0.0077 & 0.0002 & 0.0015 & 0.0002 & 0.86 & 83 & 488 & 50 & 15 & 49 & 1 & 30 & 4 & 49 & 2 \\
\hline 10TQ14-077 & 0.0515 & 0.0008 & 0.1313 & 0.0017 & 0.0185 & 0.0001 & 0.0058 & 0.0000 & 1.52 & 265 & 16 & 125 & 1 & 118.1 & 0.8 & 116.3 & 0.8 & 118.1 & 1.6 \\
\hline 10TQ14-078 & 0.0622 & 0.0013 & 0.8162 & 0.0152 & 0.0952 & 0.0008 & 0.0185 & 0.0001 & 0.20 & 682 & 26 & 606 & 8 & 586 & 1 & 371 & 2 & 586 & 8 \\
\hline 10TC & 0.0914 & 0.0013 & 9030 & 0.0345 & 0.2304 & 0.0017 & 0.0431 & 0.0004 & 1.14 & 1455 & 12 & 1383 & 9 & 1337 & 9 & 853 & 8 & 1455 & 24 \\
\hline 080 & 0.0485 & 0.0057 & 0.0912 & 0.0106 & 0.0136 & 0.0003 & 0.0039 & 0.0002 & 1.14 & 126 & 216 & 89 & 10 & 87 & 2 & 78 & 3 & 87 & 4 \\
\hline 081 & 0.0482 & 0.0017 & .1061 & 0.0037 & 0.0160 & 0.0002 & 0.0050 & 0.0001 & 0.73 & 107 & 63 & 102 & 3 & 102.2 & 9 & 100 & 1 & 102.2 & 2 \\
\hline 4-082 & 0.0538 & 0.0061 & & 0.0240 & 0.0288 & & & & 1.03 & 364 & 220 & 196 & 20 & 183 & 0 & 183 & 7 & 183 & 6 \\
\hline $10 \mathrm{TC}$ & & & & 0.0056 & 0.0133 & & & 01 & 1.14 & 278 & 112 & 92 & 5 & 85 & 1 & 88 & 2 & 35 & 2 \\
\hline 4-084 & & 8 & & 0.0014 & 0.0057 & & & 00 & 2.36 & 233 & 65 & 4 & 1 & 36.8 & 0.3 & 42 & 0.8 & 36.8 & 0.6 \\
\hline 10TC & & & & & 0.0138 & & & & 1.13 & 102 & 24 & 89 & 11 & $8 \varepsilon$ & 1 & 15 & 3 & 8 & 2 \\
\hline 4-086 & 0.0474 & .0046 & & 0.0048 & .0078 & .0001 & 0.0024 & 0.0001 & 0.87 & 69 & 183 & 50 & 5 & 50 & 0.8 & 49 & 1 & 0 & \\
\hline 887 & 0.0486 & .0025 & & 0.0020 & .0061 & 0.0001 & 0.0019 & 0.0000 & 1.50 & 129 & 92 & 41 & 2 & 39.1 & 0.4 & 39 & 0.8 & 39.1 & \\
\hline OTC & 0.0501 & 0038 & 0503 & 0.0038 & .0073 & 0.0001 & 0.0023 & 0.0001 & 0.90 & 198 & 147 & 50 & 4 & 46.8 & 0.6 & 47 & 1 & 46.8 & 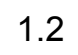 \\
\hline 39 & & & & 0.0140 & & 0007 & & 0.0002 & 2.58 & 569 & 44 & 575 & 8 & 577 & 4 & 577 & 4 & 577 & \\
\hline & 0.0484 & 0058 & 0585 & 0.0070 & 0.0088 & 0.0001 & 0.0029 & 0.0002 & 2.56 & 119 & 237 & 58 & 7 & 56.2 & 0.8 & 58 & 4 & 56.2 & 6 \\
\hline 10TQ14-091 & 0.0770 & 0.0010 & 2.0007 & 0.0216 & 0.1885 & 0.0013 & 0.0622 & 0.0007 & 4.88 & 1121 & 11 & 1116 & 7 & 1113 & 7 & 1219 & 14 & 1121 & \\
\hline
\end{tabular}




\begin{tabular}{|c|c|c|c|c|c|c|c|c|c|c|c|c|c|c|c|c|c|c|c|}
\hline 10TQ14-092 & 0.0596 & 0.0024 & 0.4824 & 0.0183 & 0.0587 & 0.0007 & 0.0179 & 0.0003 & 0.75 & 589 & 62 & 400 & 13 & 368 & 4 & 359 & 6 & 368 & 8 \\
\hline 10TQ14-093 & 0.0532 & 0.0013 & 0.2341 & 0.0053 & 0.0319 & 0.0003 & 0.0099 & 0.0001 & 1.56 & 337 & 36 & 214 & 4 & 203 & 2 & 199 & 2 & 203 & 4 \\
\hline 10TQ14-094 & 0.0514 & 0.0021 & 0.2089 & 0.0083 & 0.0295 & 0.0003 & 0.0092 & 0.0002 & 1.10 & 258 & 72 & 193 & 7 & 187 & 2 & 184 & 3 & 187 & 4 \\
\hline 10TQ14-095 & 0.0741 & 0.0011 & 1.3971 & 0.0164 & 0.1368 & 0.0010 & 0.0321 & 0.0006 & 7.53 & 1044 & 13 & 888 & 7 & 827 & 6 & 639 & 11 & 827 & 12 \\
\hline 10TQ14-096 & 0.0499 & 0.0041 & 0.0957 & 0.0077 & 0.0139 & 0.0003 & 0.0041 & 0.0001 & 0.99 & 188 & 149 & 93 & 7 & 89 & 2 & 82 & 3 & 89 & 4 \\
\hline 10TQ14-097 & 0.0530 & 0.0066 & 0.0579 & 0.0071 & 0.0079 & 0.0002 & 0.0025 & 0.0000 & 0.97 & 328 & 280 & 57 & 7 & 50.9 & 1 & 50.2 & 0.7 & 50.9 & 2 \\
\hline 10TQ14-098 & 0.0482 & 0.0046 & 0.0431 & 0.0040 & 0.0065 & 0.0001 & 0.0025 & 0.0001 & 1.81 & 111 & 169 & 43 & 4 & 41.6 & 0.8 & 51 & 2 & 41.6 & 1.6 \\
\hline 10TQ14-099 & 0.0515 & 0.0088 & 0.1104 & 0.0186 & 0.0155 & 0.0004 & 0.0062 & 0.0003 & 1.30 & 265 & 303 & 106 & 17 & 99 & 3 & 125 & 7 & 99 & 6 \\
\hline 10TQ14-100 & 0.0518 & 0.0042 & 0.1091 & 0.0087 & 0.0153 & 0.0002 & 0.0050 & 0.0001 & 0.92 & 274 & 162 & 105 & 8 & 98 & 1 & 101 & 2 & 98 & 2 \\
\hline 10TQ14-101 & 0.0482 & 0.0077 & 0.0572 & 0.0091 & 0.0086 & 0.0001 & 0.0030 & 0.0001 & 1.35 & 111 & 285 & 56 & 9 & 55.2 & 0.9 & 61 & 2 & 55.2 & 1.8 \\
\hline 10TQ14-102 & 0.0514 & 0.0076 & 0.2177 & 0.0317 & 0.0307 & 0.0008 & 0.0097 & 0.0005 & 1.18 & 261 & 276 & 200 & 26 & 195 & 5 & 196 & 11 & 195 & 10 \\
\hline 10TQ14-103 & 0.0510 & 0.0046 & 0.0566 & 0.0050 & 0.0081 & 0.0001 & 0.0027 & 0.0001 & 1.70 & 241 & 176 & 56 & 5 & 51.7 & 0.7 & 55 & 2 & 51.7 & 1.4 \\
\hline 10TQ14-104 & 0.0486 & 0.0039 & 0.0949 & 0.0075 & 0.0142 & 0.0002 & 0.0047 & 0.0001 & 1.36 & 127 & 149 & 92 & 7 & 91 & 1 & 94 & 3 & 91 & 2 \\
\hline
\end{tabular}


TABLE DR2. MAJOR ELEMENT DATA OF DIFFERENT KINDS OF MINERALS FOR THE TUFFITE FROM LIUQU FORMATION BY EPMA.

\begin{tabular}{cccccccccccccc}
\hline \hline No. & type & $\mathrm{K}_{2} \mathrm{O}$ & $\mathrm{CaO}$ & $\mathrm{TiO}_{2}$ & $\mathrm{Na}_{2} \mathrm{O}$ & $\mathrm{MgO}$ & $\mathrm{Al}_{2} \mathrm{O}_{3}$ & $\mathrm{SiO}_{2}$ & $\mathrm{Cr}_{2} \mathrm{O}_{3}$ & $\mathrm{MnO}$ & $\mathrm{FeO}$ & $\mathrm{NiO}$ & Total \\
\hline 1 & glass & 0.93 & 0.19 & 0.22 & 0.05 & 0.76 & 4.03 & 87.74 & 0.00 & 0.03 & 2.29 & 0.02 & 96.24 \\
2 & glass & 1.02 & 0.24 & 0.19 & 0.05 & 1.48 & 5.94 & 83.85 & 0.03 & 0.05 & 4.97 & 0.05 & 97.86 \\
3 & glass & 0.48 & 0.15 & 0.07 & 0.04 & 0.66 & 2.55 & 93.32 & 0.01 & 0.00 & 1.11 & 0.00 & 98.39 \\
4 & glass & 1.71 & 0.22 & 0.80 & 0.05 & 1.50 & 6.69 & 85.37 & 0.02 & 0.02 & 3.20 & 0.01 & 99.59 \\
5 & glass & 1.08 & 0.17 & 0.12 & 0.04 & 0.46 & 4.58 & 90.39 & 0.01 & 0.01 & 1.79 & 0.07 & 98.72 \\
6 & glass & 1.54 & 5.52 & 0.01 & 0.19 & 4.04 & 9.92 & 63.07 & 0.03 & 0.22 & 2.93 & 0.00 & 87.47 \\
7 & Illite & 5.92 & 0.48 & 0.81 & 0.42 & 2.31 & 31.86 & 47.72 & 0.08 & 0.06 & 3.48 & 0.02 & 93.15 \\
8 & Illite & 6.04 & 0.51 & 0.79 & 0.46 & 2.12 & 33.23 & 49.43 & 0.03 & 0.07 & 3.20 & 0.00 & 95.87 \\
9 & Chlorite & 0.46 & 0.47 & 0.15 & 0.08 & 11.45 & 22.45 & 32.32 & 0.04 & 0.43 & 21.57 & 0.01 & 89.44 \\
10 & Chlorite & 0.25 & 0.51 & 0.06 & 0.08 & 11.99 & 21.29 & 30.40 & 0.02 & 0.32 & 21.06 & 0.07 & 86.05 \\
\hline
\end{tabular}


TABLE DR3. SCORES PF THE LEAF FOSSIL PHYSIOGNOMY OF THE LIUQU, QIABULIN, NAMLING AND INDIAN SITES.

\begin{tabular}{|c|c|c|c|c|c|c|}
\hline parameters & Liuqu & Qiabulin & Namling & Gurha & Tirap & Kameng $\mathbf{R}$. \\
\hline Lobed & 0 & 1.9 & 10.3 & 0.0 & 1.3 & 0.0 \\
\hline No Teeth & 89.7 & 52.1 & 20.6 & 87.3 & 100 & 100.0 \\
\hline Regular teeth & 2.9 & 20.8 & 61.8 & 6.8 & 0 & 0.0 \\
\hline Close teeth & 0.7 & 7.3 & 57.4 & 4.0 & 0 & 0.0 \\
\hline Round teeth & 4.4 & 26 & 32.4 & 7.7 & 0 & 0.0 \\
\hline Acute teeth & 5.9 & 21.9 & 50 & 5.0 & 0 & 0.0 \\
\hline Compound teeth & 0 & 0 & 27.9 & 0.0 & 0 & 0.0 \\
\hline Nanophyll & 0 & 0 & 0 & 0.0 & 0 & 0.0 \\
\hline Leptophyll 1 & 0 & 0 & 4.5 & 0.0 & 0 & 0.0 \\
\hline Leptophyll 2 & 0 & 2.1 & 3 & 0.0 & 0 & 0.0 \\
\hline Microphyll 1 & 0 & 6.3 & 24.5 & 7.5 & 1.3 & 0.0 \\
\hline Microphyll 2 & 39 & 39.5 & 52.7 & 30.1 & 13.8 & 9.4 \\
\hline Microphyll 3 & 39.3 & 28.1 & 13.3 & 20.4 & 21.3 & 28.1 \\
\hline Mesophyll 1 & 12.7 & 13.5 & 0.9 & 16.6 & 31.3 & 45.3 \\
\hline Mesophyll 2 & 3.4 & 9.4 & 0 & 9.9 & 6.3 & 9.4 \\
\hline Mesophyll 3 & 5.5 & 1 & 0 & 15.5 & 31.3 & 7.8 \\
\hline Emarginate apex & 6.3 & 6.7 & 0 & 9.2 & 5.7 & 0.0 \\
\hline Round apex & 8.3 & 10 & 17.3 & 14.1 & 14.3 & 9.1 \\
\hline Acute apex & 33.3 & 50 & 65 & 45.8 & 14.3 & 68.2 \\
\hline Attenuate apex & 52.1 & 40 & 17.3 & 33.3 & 65.7 & 22.7 \\
\hline Cordate base & 7.1 & 14.5 & 23.5 & 12.3 & 7.3 & 0.0 \\
\hline Round base & 26.8 & 38.4 & 46.1 & 15.7 & 45.5 & 6.9 \\
\hline Acute base & 66.1 & 47.1 & 30 & 72.0 & 47.3 & 93.1 \\
\hline $\mathrm{L}: \mathrm{W}<1: 1$ & 0 & 0 & 6.3 & 0.0 & 1.3 & 0.0 \\
\hline L:W 1-2:1 & 41.4 & 47.7 & 37.5 & 37.2 & 31.6 & 47.8 \\
\hline L:W 2-3:1 & 30.1 & 25 & 41.6 & 40.9 & 40.8 & 32.6 \\
\hline L:W 3-4:1 & 20.4 & 9.1 & 7.2 & 13.2 & 27.6 & 15.2 \\
\hline $\mathrm{L}: \mathrm{W}>4: 1$ & 8.1 & 18.2 & 7.2 & 8.7 & 0 & 4.3 \\
\hline Obovate & 3.4 & 2.4 & 2.9 & 1.0 & 0 & 7.8 \\
\hline Elliptic & 94.8 & 88.1 & 66.2 & 97.3 & 97.5 & 87.5 \\
\hline Ovate & 1.7 & 9.5 & 30.9 & 1.8 & 2.5 & 4.7 \\
\hline
\end{tabular}


TABLE DR4. CLIMATE PARAMETERS PREDICTED BY CLAMP AND THEIR ASSOCIATED STATISTICAL UNCERTAINTIES.

\begin{tabular}{|c|c|c|c|c|c|c|c|c|c|c|c|c|c|c|c|}
\hline Location & $\begin{array}{l}\text { Time } \\
\text { (Ma) }\end{array}$ & $\begin{array}{c}\text { Latitude } \\
\left({ }^{\circ} \mathrm{N}\right)\end{array}$ & $\begin{array}{l}\text { Longitude } \\
\left({ }^{\circ} \mathrm{E}\right)\end{array}$ & $\begin{array}{l}\text { MAT } \\
\left({ }^{\circ} \mathrm{C}\right)\end{array}$ & $\begin{array}{l}\text { WMMT } \\
\left({ }^{\circ} \mathrm{C}\right)\end{array}$ & $\begin{array}{l}\text { CMMT } \\
\left({ }^{\circ} \mathrm{C}\right)\end{array}$ & $\begin{array}{c}\text { LSG } \\
\text { (Months) }\end{array}$ & $\begin{array}{l}\text { GSP } \\
\text { (cm) }\end{array}$ & $\begin{array}{l}\text { MMGSP } \\
\text { (cm) }\end{array}$ & $\begin{array}{l}\text { 3-WET } \\
\text { (cm) }\end{array}$ & $\begin{array}{c}\text { 3-DRY } \\
\text { (cm) }\end{array}$ & $\begin{array}{l}\mathrm{RH} \\
(\%)\end{array}$ & $\begin{array}{c}\mathrm{SH} \\
(\mathrm{g} / \mathrm{kg})\end{array}$ & $\begin{array}{l}\text { ENTHAL } \\
\text { (kJ/kg) }\end{array}$ & 3WET/3DRY \\
\hline Liuqu & $\sim 56$ & 29.197 & 87.832 & 23.8 & 28.4 & 15.3 & 12.4 & 220.5 & 21.4 & 102.6 & 20.6 & 75.9 & 13.5 & 349.6 & $5.0 \pm 2.8$ \\
\hline Qiabulin & $21-19$ & 29.335 & 88.507 & 19.1 & 27.8 & 8.5 & 10.5 & 188.1 & 18.8 & 89.6 & 19.2 & 72.2 & 11.0 & 335.7 & $4.7 \pm 2.9$ \\
\hline Namling & $\sim 15$ & 29.696 & 89.579 & 8.2 & 23.8 & -5.7 & 6.0 & 131.4 & 16.0 & 68.3 & 22.0 & 66.5 & 4.0 & 296.3 & $3.1 \pm 2.1$ \\
\hline Gurha & $55-52$ & 27.874 & 72.867 & 24.3 & 28.1 & 18.6 & 12.2 & 181.5 & 15.5 & 95.7 & 9.4 & 78.3 & 14.3 & 352.8 & $10.2 \pm 11.0$ \\
\hline Tirap & $\sim 23$ & 27.289 & 95.771 & 26.2 & 28.4 & 20.4 & 13.0 & 212.6 & 19.2 & 110.9 & 9.7 & 81.2 & 15.1 & 356.9 & $11.5 \pm 12.0$ \\
\hline Kemang R. & $\sim 13$ & 27.043 & 92.605 & 25.3 & 27.8 & 21.3 & 12.5 & 174.1 & 14.0 & 96.2 & 7.3 & 81.2 & 14.9 & 355.8 & $13.1 \pm 17.0$ \\
\hline $\begin{array}{l}\text { Uncertainty } \\
\text { (STDEV) }\end{array}$ & ---- & NA & NA & 2.3 & 2.8 & 3.6 & 1.1 & 60.6 & 6.1 & 35.8 & 9.5 & 8.4 & 1.9 & 8.7 & NA \\
\hline Shilong & Modern & 25.57 & $91 . \overline{8}$ & $17.4 \pm 2.3$ & $20.7 \pm 2.5$ & $11.5 \pm 2.2$ & $\bar{N} \bar{A}$ & NA & $\overline{N A}$ & $118.0 \pm 11.6$ & $6.8 \pm 1.6$ & $\overline{N A}$ & NA & NA & $-\overline{17.4 \pm 4.5}$ \\
\hline Lhasa & Modern & 29.3 & 88.9 & $8.0 \pm 0.8$ & $15.7 \pm 0.6$ & $-0.4 \pm 0.8$ & NA & NA & NA & $32.7 \pm 4.0$ & $0.4 \pm 0.1$ & NA & NA & NA & $81.8 \pm 10.2$ \\
\hline
\end{tabular}


TABLE DR5. PALEOELEVATIONS ESTIMATED FROM LIUQU, QIABULIN AND NAMLING FOSSIL FLORAS BY CLAMP.

\begin{tabular}{|c|c|c|c|c|c|c|}
\hline Location & $\begin{array}{l}\text { Time } \\
(\mathrm{Ma})\end{array}$ & $\begin{array}{c}\text { ENTHAL } \\
(\mathrm{kJ} / \mathrm{kg})\end{array}$ & $\begin{array}{c}\text { Sea level enthalpy } \\
(\mathrm{kJ} / \mathrm{kg})\end{array}$ & $\begin{array}{l}\text { Palaeoelevation } \\
\text { (raw) (m) }\end{array}$ & $\begin{array}{c}\text { Palaeoelevation } \\
\text { (adjusted) (m) }\end{array}$ & $\begin{array}{l}\text { Uncertainty } \\
\text { (m) }\end{array}$ \\
\hline Liuqu & -56 & 349.6 & 352.8 & 326 & 919 & 887 \\
\hline Qiabulin & $21-19$ & 335.7 & 356.9 & 2161 & 2315 & 887 \\
\hline Namling & $\sim 15$ & 296.3 & 355.8 & 6065 & 5544 & 887 \\
\hline
\end{tabular}

